\title{
Accessing Elaborated 2,1-Borazaronaphthalene Cores using Photoredox/Nickel Dual-Catalytic Functionalization
}

\author{
Matthieu Jouffroy, Geraint H. M. Davies, and Gary A. Molander* \\ Roy and Diana Vagelos Laboratories, Department of Chemistry, University of Pennsylvania, \\ 231 South $34^{\text {th }}$ Street, Philadelphia, Pennsylvania 19104-6323, United States \\ *To whom correspondence should be addressed. E-mail: gmolandr@sas.upenn.edu
}

\section{Supporting Information}

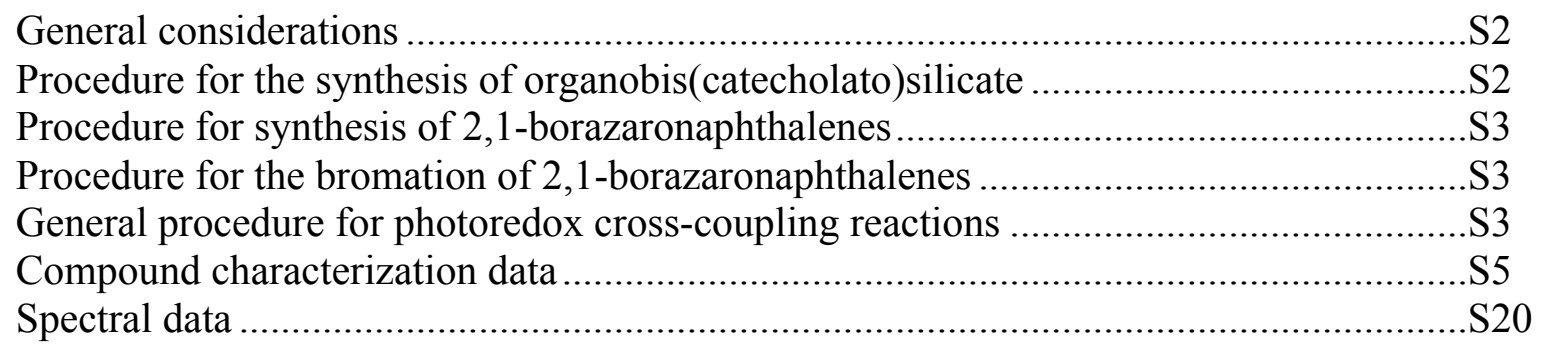




\section{General considerations}

All reactions were carried out under an inert atmosphere of nitrogen or argon in oven-dried glassware, unless otherwise noted. Conventional solvents (THF, $\mathrm{Et}_{2} \mathrm{O}, \mathrm{CH}_{2} \mathrm{Cl}_{2}$, toluene, CPME) were dried using a J. C. Meyer solvent system. DMF (99.9\%, extra dry) was used as received. $\mathrm{NEt}_{3}$ and $(i-\mathrm{Pr})_{2} \mathrm{NH}$ were distilled prior to use and stored over activated molecular sieves. Catechol $(99 \%)$ was recrystallized from heptane. $\left[\mathrm{NiCl}_{2}(\mathrm{dme})\right]$ was purchased from commercial sources, and all other reagents were purchased commercially and used as received, unless otherwise noted. Column chromatography was performed by Combiflash ${ }^{(R)}$ using RediSep Rf Gold Normal-Phase Silica ${ }^{(\mathrm{R})}$ columns. Photoredox reactions were irradiated with blue LED strips, and the temperature was controlled using an external fan. Melting points $\left({ }^{\circ} \mathrm{C}\right)$ are uncorrected. Mass spectra (ESI- or CI-TOF) were recorded using $\mathrm{CH}_{2} \mathrm{Cl}_{2}$, $\mathrm{MeCN}$ or MeOH as the solvent. NMR Spectra $\left({ }^{1} \mathrm{H},{ }^{13} \mathrm{C}\left\{{ }^{1} \mathrm{H}\right\},{ }^{11} \mathrm{~B},{ }^{19} \mathrm{~F}\left\{{ }^{1} \mathrm{H}\right\}\right)$ were performed at $298 \mathrm{~K} .{ }^{1} \mathrm{H}(500.4 \mathrm{MHz})$ and ${ }^{13} \mathrm{C}\left\{{ }^{1} \mathrm{H}\right\}(125.8 \mathrm{MHz})$ NMR chemical shifts are reported relative to internal TMS $(\delta=0.00 \mathrm{ppm})$ or to residual protiated solvent. ${ }^{11} \mathrm{~B}(128.4 \mathrm{MHz})$ and ${ }^{19} \mathrm{~F}\left\{{ }^{1} \mathrm{H}\right\}$ NMR $(470.8 \mathrm{MHz})$ chemical shifts were referenced to external $\mathrm{BF}_{3} \cdot \mathrm{Et}_{2} \mathrm{O}(0.0 \mathrm{ppm})$ and $\mathrm{CFCl}_{3}(0.0 \mathrm{ppm})$, respectively. Data are presented as follows: chemical shift $(\mathrm{ppm})$, multiplicity $(\mathrm{s}=$ singlet, $\mathrm{d}=$ doublet, $\mathrm{t}=$ triplet, $\mathrm{q}=$ quartet, $\mathrm{sept}=$ septet, $\mathrm{m}=$ multiplet, $\mathrm{br}=$ broad), coupling constant $J(\mathrm{~Hz})$ and integration. Ammonium organobis(catecholato)silicates ${ }^{1}$ and 2,1-borazaronaphthalenes ${ }^{2}$ were prepared according to literature procedures.

\section{Procedure for synthesis of organobis(catecholato)silicates}

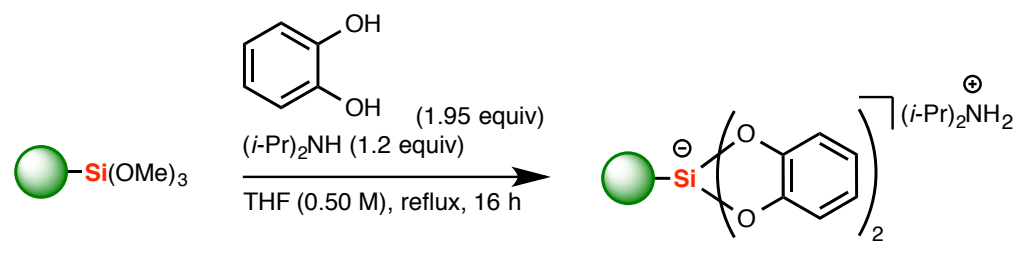

Catechol (1.95 equiv) was introduced into a microwave vial with a stirring bar. The vial was sealed with a Teflon-coated septum cap, then purged with $\mathrm{N}_{2}$ and evacuated four times. THF and $(i-\mathrm{Pr})_{2} \mathrm{NH}$ (1.2 equiv) were introduced, and the resulting light pink solution was stirred at $\mathrm{rt}$ for 15 min before addition of organotrimethoxysilane derivative (1 equiv) (Note: no base was added for the synthesis of alkylamine containing silane). The reaction mixture was heated to $75^{\circ} \mathrm{C}$ for $16 \mathrm{~h}$. The vial was then cooled to $\mathrm{rt}$, and the reaction mixture concentrated to a thick slurry before $\mathrm{Et}_{2} \mathrm{O}(0.1 \mathrm{M})$ was introduced. The mixture was then sonicated 15 min to allow the corresponding product to precipitate as a white powder. The vial was finally unsealed, and the precipitate was collected by vacuum filtration. The filter cake was washed with $\mathrm{Et}_{2} \mathrm{O}$ to afford silicate 11. If required, the product was further purified by dissolving in $\mathrm{CH}_{2} \mathrm{Cl}_{2}$ followed by precipitation with pentane and vacuum filtration.

\footnotetext{
${ }^{1}$ (a) Jouffroy, M.; Primer, D. N.; Molander, G. A. J. Am. Chem. Soc. 2016, 138, 475. (b) Patel, N. R.; Kelly, C. B.; Jouffroy, M.; Molander, G. A. Org. Lett., DOI: 10.1021/acs.orglett.6b00024.

${ }^{2}$ Wisniewski, S. R.; Guenther, C. L.; Argintaru, O. A.; Molander, G. A. J. Org. Chem. 2014, 79, 365.
} 


\section{Procedure for synthesis of 2,1-borazaronaphthalenes}

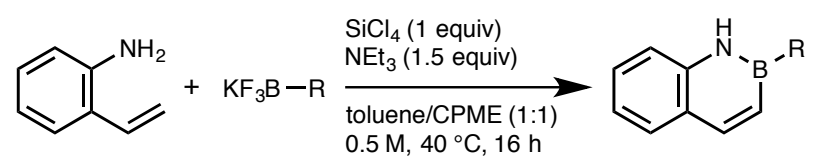

Aryltrifluoroborate (1.0 equiv) was introduced into a microwave vial with a stirring bar. The vial was sealed with a Teflon-coated septum cap, then purged with $\mathrm{N}_{2}$ and evacuated four times. CPME/toluene mixture $(0.5 \mathrm{M}, 1: 1, v / v)$ and 2-aminostyrene (1.2 equiv) were introduced via syringe, followed by $\mathrm{NEt}_{3}$ (1.5 equiv) and $\mathrm{SiCl}_{4}$ (1.0 equiv). The resulting suspension was heated to $40{ }^{\circ} \mathrm{C}$ under vigorous stirring for $16 \mathrm{~h}$. The vial was finally cooled to $\mathrm{rt}$, and the reaction mixture filtered through a plug of silica and flushed with hexanes/EtOAc mixture (20 times solvent volume, 4:1, v/v). Solvents were removed in vacuo to obtain product $\mathbf{1 0}$ in pure form. If required, the residue was further purified by column chromatography on silica gel, eluting with $\mathrm{CH}_{2} \mathrm{Cl}_{2}$ and hexanes.

\section{Procedure for the bromation of 2,1-borazaronaphthalenes}

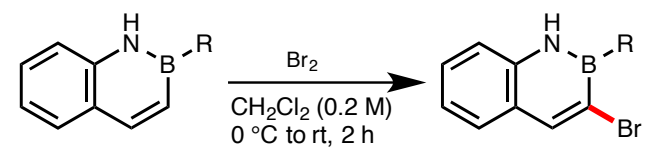

The 2,1-borazaronaphthalenes 10 (1.0 equiv) were introduced into a round-bottom flask equipped with a stirring bar. The flask was sealed with a rubber septum, then purged with inert gas and evacuated four times. $\mathrm{CH}_{2} \mathrm{Cl}_{2}(0.2 \mathrm{M})$ was introduced, and the flask was cooled to $0{ }^{\circ} \mathrm{C}$ before $\mathrm{Br}_{2}\left(1.1\right.$ equiv) in $\mathrm{CH}_{2} \mathrm{Cl}_{2}(0.2 \mathrm{M})$ was added over $2 \mathrm{~h}$. After addition, the reaction completion was monitored by HPLC. The crude reaction mixture was condensed in vacuo then purified by column chromatography on silica gel, eluting with $\mathrm{CH}_{2} \mathrm{Cl}_{2}$ and hexanes to afford product $\mathbf{1}$ in pure form.

\section{General procedure for photoredox cross-coupling reactions}

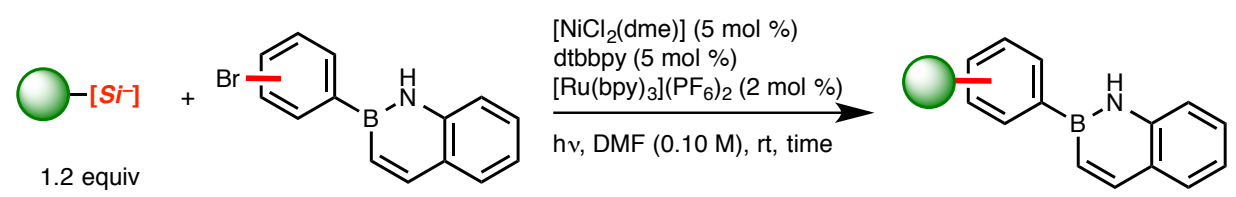

0.5 mmol scale reaction: To an $8 \mathrm{~mL}$ clear glass vial equipped with a Teflon-coated magnetic stir bar was added 4,4'-di-tert-butyl-2,2'-bipyridine $(6.7 \mathrm{mg}, 0.025 \mathrm{mmol})$, and $\left[\mathrm{NiCl}_{2}(\mathrm{dme})\right]$ (5.5 mg, $\left.0.025 \mathrm{mmol}\right)$. The vial was capped and purged with nitrogen, then 1.5 $\mathrm{mL}$ of THF was introduced. The resulting suspension was heated briefly with a heat gun until the nickel complex and ligand were fully solubilized, yielding a pale green solution. The solution was cooled in an ice bath, resulting in the immediate precipitation of an evergreen solid. Solvents were then evaporated in vacuo to give a fine coating of the ligated nickel complex. Once dry, brominated azaborine 1 ( $0.5 \mathrm{mmol}, 1.0$ equiv) (liquid aryl bromides were added at the time of solvent addition), organosilicates (0.6 mmol, 1.2 equiv), and $\left[\mathrm{Ru}(\mathrm{bpy})_{3}\right]\left(\mathrm{PF}_{6}\right)_{2}(8.6 \mathrm{mg}, 0.01 \mathrm{mmol})$ were added in succession. The vial was then capped and purged four times. Under inert atmosphere, DMF $(5 \mathrm{~mL})$ was introduced. The vial containing all the reagents was further sealed with parafilm and stirred approximately $4 \mathrm{~cm}$ away from the LED strips (Figure S-1). A fan was blown across the reaction setup to suppress the heat generated by the latter (the reaction temperatures were estimated to be $\sim 30{ }^{\circ} \mathrm{C}$ ). After 
$12 \mathrm{~h}$, an aliquot was taken and analyzed by HPLC to monitor reaction completion. The crude reaction mixture was poured into a separatory funnel and diluted with $\mathrm{H}_{2} \mathrm{O}(20 \mathrm{~mL})$. The resulting suspension was extracted with EtOAc $(3 \times 10 \mathrm{~mL})$, and the combined organic extracts were washed with a saturated solution of $\mathrm{Na}_{2} \mathrm{CO}_{3}(2 \times 20 \mathrm{~mL})$ then $\mathrm{H}_{2} \mathrm{O}(20 \mathrm{~mL})$, dried $\left(\mathrm{MgSO}_{4}\right)$ and concentrated. The residue was purified by column chromatography on silica gel, eluting with EtOAc and hexanes, to obtain products in pure form. In the case of primary and secondary alkylamine-containing compounds, the residue was purified by column chromatography on silica gel, eluting with $\mathrm{MeOH}$ and $\mathrm{CH}_{2} \mathrm{Cl}_{2}$ containing $\mathrm{NH}_{4} \mathrm{OH}(1$ $\%, v / v)$.
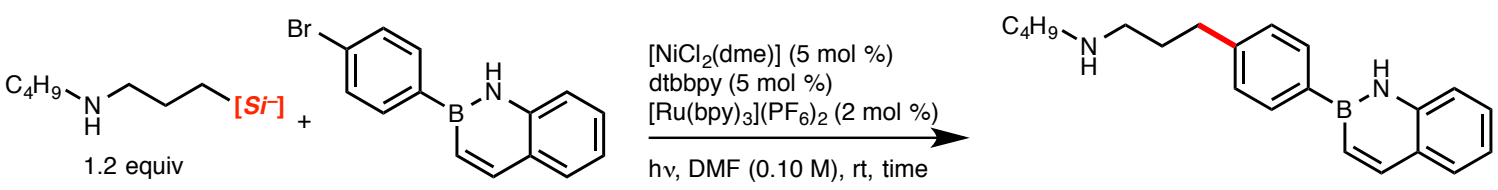

Gram scale reaction: To a $100 \mathrm{~mL}$ round bottom flask equipped with a Teflon-coated magnetic stir bar was added $\left[\mathrm{NiCl}_{2}(\mathrm{dme})\right](39 \mathrm{mg}, 0.18 \mathrm{mmol})$ and 4,4'-di-tert-butyl-2,2'bipyridine $(47 \mathrm{mg}, 0.18 \mathrm{mmol})$. The flask was capped and purged with nitrogen, then $3.0 \mathrm{~mL}$ of THF was introduced. The resulting suspension was heated briefly with a heat gun until the nickel and ligand were fully solubilized, yielding a pale green solution. The solution was cooled in an ice bath, resulting in the immediate precipitation of an evergreen solid. Solvents were then evaporated in vacuo to give a fine coating of the ligated nickel complex. Once dry, azaborine 1a (1.000 g, $3.52 \mathrm{mmol})$, bis(catecholato)3-(butylammonio)propyl silicate 11d $(1.949 \mathrm{~g}, 4.23 \mathrm{mmol})$ and $\left[\mathrm{Ru}(\mathrm{bpy})_{3}\right]\left(\mathrm{PF}_{6}\right)_{2}(61 \mathrm{mg}, 0.07 \mathrm{mmol})$ were added in succession. The vial was then capped and purged four times. Under inert atmosphere, DMF (35 mL) was introduced. The vial containing all the reagents was further sealed with parafilm and stirred in the presence of coiled blue LEDs (Figure S-1). A fan was blown across the reaction setup to suppress the heat generated by the LEDs, stabilizing at $30^{\circ} \mathrm{C}$ after $1 \mathrm{~h}$. Reaction completion was monitored by sampling the reaction mixture and analyzing by HPLC. After completion (4 h), the crude reaction mixture was diluted with EtOAc $(40 \mathrm{~mL})$, filtered through approximately $6 \mathrm{~cm} \times 4 \mathrm{~cm}$ cylindrical plug of Celite, washing with EtOAc $(40 \mathrm{~mL})$. The resulting solution was concentrated, retaken in EtOAc $(50 \mathrm{~mL})$, poured into a separatory funnel and washed with a saturated solution of $\mathrm{Na}_{2} \mathrm{CO}_{3}(2 \times 40 \mathrm{~mL})$ then $\mathrm{H}_{2} \mathrm{O}(40 \mathrm{~mL})$, dried $\left(\mathrm{MgSO}_{4}\right)$ and concentrated. The residue was purified by column chromatography on silica gel, eluting with $\mathrm{MeOH}$ and $\mathrm{CH}_{2} \mathrm{Cl}_{2}$ containing $\mathrm{NH}_{4} \mathrm{OH}(1 \%, v / v)$, to obtain azaborine $\mathbf{2} \mathbf{m}$ in pure form $(1070 \mathrm{mg}, 96 \%)$.
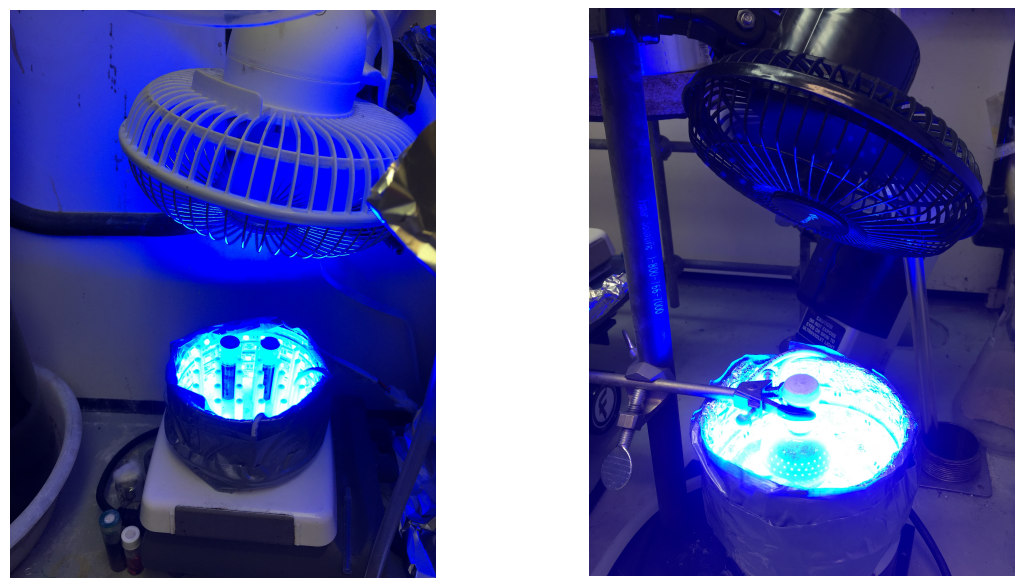

Figure $\mathbf{S}-1.0 .5 \mathrm{mmol}$ (left) and gram (right) scale photoredox cross-coupling reaction set-up. 


\section{Compound Characterization Data}<smiles>Brc1ccc(B2C=Cc3ccccc3N2)cc1</smiles>

2-(4-Bromophenyl)-2,1-borazanaphthalene (1a): obtained as an off white powder $(2.05 \mathrm{~g}$, $72 \%, 10.00 \mathrm{mmol} \mathrm{scale}$ ); $\mathrm{mp}=158{ }^{\circ} \mathrm{C} ;{ }^{1} \mathrm{H}$ NMR (DMSO- $d_{6}, 500.4 \mathrm{MHz}$ ): $\delta 10.5$ (br s, $1 \mathrm{H}$ ), $8.15(\mathrm{~d}, J=12.0 \mathrm{~Hz}, 1 \mathrm{H}), 8.02(\mathrm{~d}, J=8.2 \mathrm{~Hz}, 2 \mathrm{H}), 7.72-7.64(\mathrm{~m}, 4 \mathrm{H}), 7.47$ (ddd, $J=7.6$, $7.5,1.3 \mathrm{~Hz}, 1 \mathrm{H}), 7.22$ (dd, $J=11.6,1.3 \mathrm{~Hz}, 1 \mathrm{H}), 7.17$ (ddd, $J=7.4,7.5,1.3 \mathrm{~Hz}, 1 \mathrm{H}$ ) ppm; ${ }^{13} \mathrm{C}\left\{{ }^{1} \mathrm{H}\right\}$ NMR (DMSO- $\left.d_{6}, 125.8 \mathrm{MHz}\right): \delta 145.9,141.2,135.8,131.2,129.5,128.8,125.5$, 123.9, 121.1, $119.1 \mathrm{ppm} ;{ }^{11} \mathrm{~B}$ NMR (acetone, $128.4 \mathrm{MHz}$ ): $\delta 34.0 \mathrm{ppm}$; IR: $v=1612,1576$, 1557, 1490, 1434, 1390, 1345, 1280, 1206, 1134, 1077, 1067, 1007, 974, 808, $758 \mathrm{~cm}^{-1}$; HRMS (ESI) $\mathrm{m} / \mathrm{z}$ calc. for $\mathrm{C}_{14} \mathrm{H}_{11} \mathrm{BBrClN}[\mathrm{M}+\mathrm{Cl}]^{-} 317.9856$, found 317.9857 .<smiles>Brc1cccc(B2C=Cc3ccccc3N2)c1</smiles>

2-(3-Bromophenyl)-2,1-borazanaphthalene (1b): obtained as an off white powder $(1.11 \mathrm{~g}$, $56 \%, 7.00 \mathrm{mmol}$ scale); $\mathrm{mp}=75{ }^{\circ} \mathrm{C} ;{ }^{1} \mathrm{H}$ NMR (acetone- $d_{6}, 500.4 \mathrm{MHz}$ ): $\delta 9.93$ (br s, $1 \mathrm{H}$ ), $8.21(\mathrm{~d}, J=10.7 \mathrm{~Hz}, 1 \mathrm{H}), 8.17(\mathrm{~s}, 1 \mathrm{H}), 8.00(\mathrm{~d}, J=6.7 \mathrm{~Hz}, 1 \mathrm{H}), 7.70(\mathrm{~d}, J=8.1 \mathrm{~Hz}, 1 \mathrm{H})$, $7.68(\mathrm{~d}, J=8.1 \mathrm{~Hz}, 1 \mathrm{H}), 7.58(\mathrm{~d}, J=7.4 \mathrm{~Hz}, 1 \mathrm{H}), 7.47(\mathrm{ddd}, J=7.4,7.4,1.2 \mathrm{~Hz}, 1 \mathrm{H}), 7.39$ $(\mathrm{t}, J=7.7 \mathrm{~Hz}, 1 \mathrm{H}), 7.27(\mathrm{dd}, J=7.4,1.5 \mathrm{~Hz}, 1 \mathrm{H}), 7.20(\mathrm{ddd}, J=8.2,6.7,1.0 \mathrm{~Hz}, 1 \mathrm{H}) \mathrm{ppm}$; ${ }^{13} \mathrm{C}\left\{{ }^{1} \mathrm{H}\right\}$ NMR (acetone- $d_{6}, 125.8 \mathrm{MHz}$ ): $\delta 145.8,140.9,135.4,132.0,131.9,130.0,129.1$, 128.4, 125.7, 122.5, 121.0, $118.6 \mathrm{ppm} ;{ }^{11} \mathrm{~B}$ NMR (acetone, $128.4 \mathrm{MHz}$ ): $\delta 33.7 \mathrm{ppm}$; IR: $v=$ 1612, 1594, 1560, 1550, 1482, 1432, 1394, 1347, 1275, 1211, 1121, 1074, 987, 816, 786, 763 $\mathrm{cm}^{-1}$; HRMS (ESI) m/z calc. for $\mathrm{C}_{14} \mathrm{H}_{10} \mathrm{BBrN}[\mathrm{M}-\mathrm{H}]^{-} 282.0090$, found 282.0100.<smiles>Brc1ccccc1B1C=Cc2ccccc2N1</smiles>

2-(2-Bromophenyl)-2,1-borazanaphthalene (1c): obtained as a viscous colorless oil (857 $\mathrm{mg}, 51 \%, 6.00 \mathrm{mmol} \mathrm{scale}) ;{ }^{1} \mathrm{H} \mathrm{NMR}\left(\mathrm{CDCl}_{3}, 500.4 \mathrm{MHz}\right): \delta 8.42$ (br s, $\left.1 \mathrm{H}\right), 8.15$ (d, $J=$ $11.4 \mathrm{~Hz}, 1 \mathrm{H}), 7.68(\mathrm{~d}, J=8.0 \mathrm{~Hz}, 1 \mathrm{H}), 7.64-7.59(\mathrm{~m}, 2 \mathrm{H}), 7.46$ (ddd, $J=7.5,7.6,1.3 \mathrm{~Hz}, 1$ H), 7.37 (ddd, $J=7.5,7.4,1.0 \mathrm{~Hz}, 1 \mathrm{H}), 7.33(\mathrm{~d}, J=8.1 \mathrm{~Hz}, 1 \mathrm{H}), 7.27-7.20(\mathrm{~m}, 2 \mathrm{H}), 7.16$ (dd, $J=11.7,2.3 \mathrm{~Hz}, 1 \mathrm{H}) \mathrm{ppm} ;{ }^{13} \mathrm{C}\left\{{ }^{1} \mathrm{H}\right\} \mathrm{NMR}\left(\mathrm{CDCl}_{3}, 125.8 \mathrm{MHz}\right): \delta 145.3,139.6,135.7$, 132.6, 130.3, 129.5, 128.5, 127.4, 126.9, 12.4, 121.5, 118.5 ppm; ${ }^{11} \mathrm{~B}$ NMR (acetone, 128.4 MHz): $\delta 34.9$ ppm; IR: $v=1614,1595,1583,1559,1434,1414,1386,1346,1285,1211$, 1200, 1015, 978, 807, 748, $727 \mathrm{~cm}^{-1}$; HRMS (ESI) m/z calc. for $\mathrm{C}_{14} \mathrm{H}_{12} \mathrm{BBrN}[\mathrm{M}+\mathrm{H}]^{+}$ 284.0246 , found 284.0250 . 
<smiles>BrC1=Cc2ccccc2NB1c1ccccc1</smiles>

3-Bromo-2-phenyl-2,1-borazanaphthalene (1d): obtained as an off white powder (542 mg, $95 \%, 2.00 \mathrm{mmol}$ scale) from 2-phenyl-2,1-borazanaphthalene; ${ }^{2} \mathrm{mp}=81{ }^{\circ} \mathrm{C} ;{ }^{1} \mathrm{H} \mathrm{NMR}$ $\left(\mathrm{CDCl}_{3}, 500.4 \mathrm{MHz}\right): \delta 8.46(\mathrm{~s}, 1 \mathrm{H}), 8.04(\mathrm{br} \mathrm{s}, 1 \mathrm{H}), 7.93-7.88(\mathrm{~m}, 2 \mathrm{H}), 7.62(\mathrm{~d}, J=7.8 \mathrm{~Hz}$, $1 \mathrm{H}), 7.50-7.46(\mathrm{~m}, 4 \mathrm{H}), 7.31(\mathrm{~d}, J=8.3 \mathrm{~Hz}, 1 \mathrm{H}), 7.24(\mathrm{t}, J=7.6 \mathrm{~Hz}, 1 \mathrm{H}) \mathrm{ppm}$. Characterization data for this compound matched that reported in the literature. ${ }^{3}$<smiles>FC(F)(F)c1ccc(B2Nc3ccccc3C=C2Br)cc1</smiles>

3-Bromo-2-(4-(trifluoromethyl)phenyl)-2,1-borazanaphthalene (1e): obtained as an off white powder (294 mg, 67\%, $1.25 \mathrm{mmol}$ scale) from $10 \mathrm{a} ; \mathrm{mp}=104{ }^{\circ} \mathrm{C} ;{ }^{1} \mathrm{H}$ NMR (acetone- $d_{6}$, $500.4 \mathrm{MHz}$ ): $\delta 10.07($ br s, $1 \mathrm{H}), 8.55(\mathrm{~s}, 1 \mathrm{H}), 8.07(\mathrm{~d}, J=7.9 \mathrm{~Hz}, 2 \mathrm{H}), 7.77-7.67(\mathrm{~m}, 4 \mathrm{H})$, 7.55-7.51 (m, $1 \mathrm{H}), 7.28-7.24(\mathrm{~m}, 1 \mathrm{H}) \mathrm{ppm} ;{ }^{13} \mathrm{C}\left\{{ }^{1} \mathrm{H}\right\}$ NMR (acetone- $\left.d_{6}, 125.8 \mathrm{MHz}\right): \delta$ $147.0139 .9,134.1,130.1$ (q, $J=31.7 \mathrm{~Hz}), 129.0,128.6,125.1,124.6$ (q, $J=270.9 \mathrm{~Hz}), 123.8$ $(\mathrm{q}, J=3.8 \mathrm{~Hz}), 122.0,118.7 \mathrm{ppm} ;{ }^{19} \mathrm{~F}\left\{{ }^{1} \mathrm{H}\right\}$ NMR (acetone- $d_{6}, 470.8 \mathrm{MHz}$ ): $\delta-63.1 \mathrm{ppm} ;{ }^{11} \mathrm{~B}$ NMR (acetone, $128.4 \mathrm{MHz}$ ): $\delta 33.4$ ppm; IR: $v=3321,1554,1328,1225,1166,1156,1130$, 1118, 1104, 1070, 1007, 919, 847, 833, 761, 747, $719 \mathrm{~cm}^{-1}$; HRMS (ESI) m/z calc. for $\mathrm{C}_{15} \mathrm{H}_{9} \mathrm{BBrF}_{3} \mathrm{~N}[\mathrm{M}-\mathrm{H}]^{-}$349.9964, found 349.9955.<smiles>BrC1=Cc2ccccc2NB1c1ccc2c(c1)OCCO2</smiles>

3-Bromo-2-(2,3-dihydro-1,4-benzodioxin-6-yl)-2,1-borazanaphthalene (1f): obtained as an off white powder (99 mg, 23\%, $1.25 \mathrm{mmol} \mathrm{scale)} \mathrm{from} \mathbf{1 0 b} ; \mathrm{mp}=155{ }^{\circ} \mathrm{C}$; ${ }^{1} \mathrm{H}$ NMR (acetone$\left.d_{6}, 500.4 \mathrm{MHz}\right): \delta 9.86($ br s, $1 \mathrm{H}), 8.49(\mathrm{~s}, 1 \mathrm{H}), 7.72(\mathrm{~d}, J=7.8 \mathrm{~Hz}, 1 \mathrm{H}), 7.62(\mathrm{~d}, J=8.4 \mathrm{~Hz}$, $1 \mathrm{H}), 7.50(\mathrm{ddd}, J=8.2,7.1,1.3 \mathrm{~Hz}, 1 \mathrm{H}), 7.24(\mathrm{ddd}, J=8.2,7.0,1.1 \mathrm{~Hz}, 1 \mathrm{H}), 7.09(\mathrm{dd}, J=$ 4.8, $3.8 \mathrm{~Hz}, 1 \mathrm{H}), 6.90-6.83(\mathrm{~m}, 2 \mathrm{H}), 4.25(\mathrm{~s}, 4 \mathrm{H}) \mathrm{ppm} ;{ }^{13} \mathrm{C}\left\{{ }^{1} \mathrm{H}\right\}$ NMR (acetone- $d_{6}, 125.8$ $\mathrm{MHz}): \delta 145.9,145.8,143.3,139.7,128.7,128.5,126.1,124.9,121.5,120.7,118.4,117.7$, 64.2, $64.1 \mathrm{ppm} ;{ }^{11} \mathrm{~B}$ NMR (acetone, $128.4 \mathrm{MHz}$ ): $\delta 34.5 \mathrm{ppm}$; IR: $v=3324,1556,1458$, 1441, 1422, 1286, 1249, 1211, 1200, 1081, 1039, 939, 922, 901, 881, 827, $771 \mathrm{~cm}^{-1}$; HRMS (ESI) $\mathrm{m} / \mathrm{z}$ calc. for $\mathrm{C}_{17} \mathrm{H}_{14} \mathrm{BBrNO}_{4}[\mathrm{M}+\mathrm{HCOO}]^{-} 386.0207$, found 386.0199.<smiles>Fc1cccc(F)c1B1Nc2ccccc2C=C1Br</smiles>

3-Bromo-2-(2,6-difluorophenyl)-2,1-borazanaphthalene (1g): obtained as an off white powder (241 mg, 60\%, $1.25 \mathrm{mmol} \mathrm{scale}$ ) from $10 \mathrm{c} ; \mathrm{mp}=173{ }^{\circ} \mathrm{C} ;{ }^{1} \mathrm{H}$ NMR (acetone- $d_{6}, 500.4$ MHz): $\delta 10.37$ (br s, $1 \mathrm{H}), 8.59(\mathrm{~s}, 1 \mathrm{H}), 7.79(\mathrm{~d}, J=7.7 \mathrm{~Hz}, 1 \mathrm{H}), 7.69(\mathrm{~d}, J=8.6 \mathrm{~Hz}, 1 \mathrm{H})$, 7.56 (ddd, $J=8.3,7.3,1.4 \mathrm{~Hz}, 1 \mathrm{H}), 7.54-7.44(\mathrm{~m}, 1 \mathrm{H}), 7.31$ (ddd, $J=8.1,7.2,1.0 \mathrm{~Hz}, 1 \mathrm{H}$ ), 7.08-7.00 (m, $2 \mathrm{H}) \mathrm{ppm} ;{ }^{13} \mathrm{C}\left\{{ }^{1} \mathrm{H}\right\}$ NMR (acetone- $\left.d_{6}, 125.8 \mathrm{MHz}\right): \delta 164.5(\mathrm{dd}, J=242.5$,

\footnotetext{
${ }^{3}$ Molander, G. A.; Wisniewski, S. R. J. Org. Chem. 2014, 79, 6663.
} 
$13.9 \mathrm{~Hz}), 146.1,139.4,131.7(\mathrm{t}, J=10.0 \mathrm{~Hz}), 129.0,128.7,125.3,122.2,118.6,110.7(\mathrm{dd}, J$ $=22.1,5.9 \mathrm{~Hz}$ ) ppm; ${ }^{19} \mathrm{~F}$ NMR (acetone- $d_{6}, 470.8 \mathrm{MHz}$ ): $\delta-102.9 \mathrm{ppm} ;{ }^{11} \mathrm{~B}$ NMR (acetone, $128.4 \mathrm{MHz}): \delta 33.1 \mathrm{ppm}$; IR: $v=3339,1616,1596,1557,1450,1427,1345,1221,1210$, 1156, 1018, 978, 910, 781, 764, $745 \mathrm{~cm}^{-1}$; HRMS (CI) m/z calc. for $\mathrm{C}_{14} \mathrm{H}_{9} \mathrm{BBrF}_{2} \mathrm{~N}[\mathrm{M}]^{+}$ 318.9979 , found 318.9991 .<smiles>Clc1ccc(B2Nc3ccccc3C=C2Br)cc1</smiles>

3-Bromo-2-(4-chlorophenyl)-2,1-borazanaphthalene (1h): obtained as an off white powder (310 mg, 78\%, $1.25 \mathrm{mmol}$ scale) from 10d; mp $=77{ }^{\circ} \mathrm{C} ;{ }^{1} \mathrm{H}$ NMR (acetone- $d_{6}, 500.4 \mathrm{MHz}$ ): $\delta$ 9.88 (br s, $1 \mathrm{H}), 8.48(\mathrm{~s}, 1 \mathrm{H}), 7.91(\mathrm{~d}, J=8.3 \mathrm{~Hz}, 2 \mathrm{H}), 7.67-7.64(\mathrm{~m}, 2 \mathrm{H}), 7.49$ (ddd, $J=$ 8.3, 7.1, $1.3 \mathrm{~Hz}, 1 \mathrm{H}), 7.44(\mathrm{~d}, J=8.3 \mathrm{~Hz}, 2 \mathrm{H}), 7.22$ (ddd, $J=8.1,7.1,1.3 \mathrm{~Hz}, 1 \mathrm{H}) \mathrm{ppm} ;{ }^{13} \mathrm{C}$ $\left\{{ }^{1} \mathrm{H}\right\}$ NMR (acetone- $\left.d_{6}, 125.8 \mathrm{MHz}\right): \delta 146.9,140.0,135.3,134.8,129.0,128.5,127.5,125.0$, 121.8, $118.6 \mathrm{ppm} ;{ }^{11} \mathrm{~B}$ NMR (acetone, $128.4 \mathrm{MHz}$ ): $\delta 33.6 \mathrm{ppm}$; IR: $v=3369,1614,1589$, 1554, 1421, 1388, 1340, 1227, 1201, 1087, 1006, 911, 845, 818, 756, $742 \mathrm{~cm}^{-1}$; HRMS (ESI) $\mathrm{m} / \mathrm{z}$ calc. for $\mathrm{C}_{14} \mathrm{H}_{9} \mathrm{BBrClN}[\mathrm{M}-\mathrm{H}]^{-} 315.9700$, found 315.9690 .

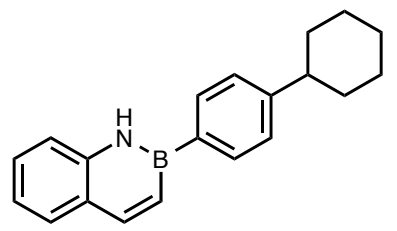

2-(4-Cyclohexylphenyl)-2,1-borazanaphthalene (2a): obtained as a white powder (139 mg, 97\%); $\mathrm{mp}=145^{\circ} \mathrm{C} ;{ }^{1} \mathrm{H} \mathrm{NMR}\left(\mathrm{CDCl}_{3}, 500.4 \mathrm{MHz}\right): \delta 8.12(\mathrm{~d}, J=10.5 \mathrm{~Hz}, 2 \mathrm{H}), 7.87(\mathrm{~d}, J=$ $8.4 \mathrm{~Hz}, 2 \mathrm{H}), 7.66(\mathrm{~d}, J=8.4 \mathrm{~Hz}, 1 \mathrm{H}), 7.44(\mathrm{dd}, J=7.4,7.4 \mathrm{~Hz}, 1 \mathrm{H}), 7.37-7.32(\mathrm{~m}, 3 \mathrm{H})$, 7.30-7.26 (m, $1 \mathrm{H}$ ), 7.19 (ddd, $J=7.6,7.4,1.1 \mathrm{~Hz}, 1 \mathrm{H}), 2.62-2.53$ (m, $1 \mathrm{H}), 1.96-1.85$ (m, 4 $\mathrm{H}), 1.78(\mathrm{~d}, J=6.0 \mathrm{~Hz}, 1 \mathrm{H}), 1.52-1.37(\mathrm{~m}, 4 \mathrm{H}), 1.34-1.27(\mathrm{~m}, 1 \mathrm{H}) \mathrm{ppm} ;{ }^{13} \mathrm{C}\left\{{ }^{1} \mathrm{H}\right\} \mathrm{NMR}$ (acetone- $\left.d_{6}, 125.8 \mathrm{MHz}\right): \delta 149.3,145.1,141.1,133.2,129.1,128.1,126.4,125.6,120.5$, $118.5,44.5,34.2,26.6,25.9 \mathrm{ppm} ;{ }^{11} \mathrm{~B}$ NMR (acetone, $\left.128.4 \mathrm{MHz}\right): \delta 34.6 \mathrm{ppm}$; IR: $v=2920$, 2849, 1612, 1596, 1564, 1439, 1404, 1388, 1347, 1284, 1218, 1174, 978, 808, 755, $737 \mathrm{~cm}^{-1}$; HRMS (ESI) $\mathrm{m} / \mathrm{z}$ calc. for $\mathrm{C}_{20} \mathrm{H}_{23} \mathrm{BN}[\mathrm{M}+\mathrm{H}]^{+}$288.1924, found 288.1924.

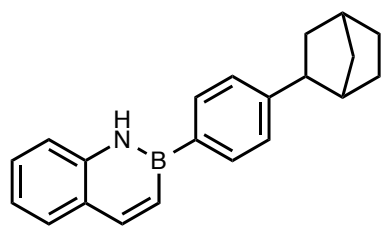

2-(4-(exo-Bicyclo[2.2.1]heptan-2-yl)phenyl)-2,1-borazanaphthalene (2b): obtained as a white powder $(143 \mathrm{mg}, 96 \%) ; \mathrm{mp}=159{ }^{\circ} \mathrm{C} ;{ }^{1} \mathrm{H}$ NMR (acetone- $\left.d_{6}, 500.4 \mathrm{MHz}\right): \delta 9.67$ (br s, $1 \mathrm{H}), 8.15(\mathrm{~d}, J=12.5 \mathrm{~Hz}, 1 \mathrm{H}), 7.96(\mathrm{~d}, J=7.2 \mathrm{~Hz}, 2 \mathrm{H}), 7.69-7.63(\mathrm{~m}, 2 \mathrm{H}), 7.43(\mathrm{dd}, J=$ 7.2, $7.9 \mathrm{~Hz}, 1 \mathrm{H}), 7.33-7.24(\mathrm{~m}, 3 \mathrm{H}), 7.16(\mathrm{dd}, J=6.6,7.2 \mathrm{~Hz}, 1 \mathrm{H}), 2.82-2.76(\mathrm{~m}, 1 \mathrm{H})$, 2.37-2.34 (m, $2 \mathrm{H}), 1.82-1.75(\mathrm{~m}, 1 \mathrm{H}), 1.73-1.68(\mathrm{~m}, 1 \mathrm{H}), 1.64-1.54(\mathrm{~m}, 3 \mathrm{H}), 1.43-1.36$ $(\mathrm{m}, 1 \mathrm{H}), 1.31-1.28(\mathrm{~m}, 1 \mathrm{H}), 1.21-1.18(\mathrm{~m}, 1 \mathrm{H}) \mathrm{ppm} ;{ }^{13} \mathrm{C}\left\{{ }^{1} \mathrm{H}\right\}$ NMR (acetone- $d_{6}, 125.8$ $\mathrm{MHz}): \delta 148.8,145.1,141.1,133.1,129.0,128.1,126.6,125.6,120.5,118.4,47.2,42.9,38.6$, 36.6, 35.6, 30.2, $28.5 \mathrm{ppm} ;{ }^{11} \mathrm{~B}$ NMR (acetone, $128.4 \mathrm{MHz}$ ): $\delta 34.0 \mathrm{ppm}$; IR: $v=2947,2866$, $1612,1594,1440,1403,1388,1345,1284,1209,1143,978,941,806,756,697 \mathrm{~cm}^{-1}$; HRMS (ESI) $\mathrm{m} / \mathrm{z}$ calc. for $\mathrm{C}_{21} \mathrm{H}_{21} \mathrm{BN}[\mathrm{M}-\mathrm{H}]^{-} 298.1767$, found 298.1763 . 


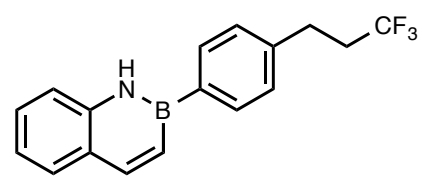

2-(4-(3,3,3-Trifluoropropyl)phenyl)-2,1-borazanaphthalene (2c): obtained as a white powder $\left(138 \mathrm{mg}, 92 \%\right.$ ) using silicate $11 \mathrm{a} ; \mathrm{mp}=151{ }^{\circ} \mathrm{C} ;{ }^{1} \mathrm{H}$ NMR (acetone- $\left.d_{6}, 500.4 \mathrm{MHz}\right): \delta$ 9.73 (br s, $1 \mathrm{H}), 8.17$ (d, $J=11.3 \mathrm{~Hz}, 1 \mathrm{H}), 8.00(\mathrm{~d}, J=7.7 \mathrm{~Hz}, 2 \mathrm{H}), 7.70-7.63(\mathrm{~m}, 2 \mathrm{H}), 7.44$ (dd, $J=7.5,7.6 \mathrm{~Hz}, 1 \mathrm{H}), 7.39$ (d, $J=8.4 \mathrm{~Hz}, 2 \mathrm{H}), 7.30-7.25(\mathrm{~m}, 1 \mathrm{H}), 7.17$ (dd, $J=7.4,7.4$ $\mathrm{Hz}, 1 \mathrm{H}), 2.95-2.89(\mathrm{~m}, 2 \mathrm{H}), 2.62-2.50(\mathrm{~m}, 2 \mathrm{H}) \mathrm{ppm} ;{ }^{13} \mathrm{C}\left\{{ }^{1} \mathrm{H}\right\} \mathrm{NMR}\left(\mathrm{CDCl}_{3}, 125.8 \mathrm{MHz}\right)$ : $\delta 145.5,140.5,140.0,133.1,129.4,128.3,128.1,126.7(\mathrm{q}, J=276.3 \mathrm{~Hz}), 125.7,121.0$, $118.1,35.5(\mathrm{q}, J=28.7 \mathrm{~Hz}), 28.2(\mathrm{q}, J=2.73 \mathrm{~Hz}) \mathrm{ppm} ;{ }^{11} \mathrm{~B}$ NMR (acetone, $\left.128.4 \mathrm{MHz}\right): \delta$ $33.9 \mathrm{ppm} ;{ }^{19} \mathrm{~F}\left\{{ }^{1} \mathrm{H}\right\}$ NMR (acetone- $d_{6}, 470.8 \mathrm{MHz}$ ): $-67.0 \mathrm{ppm}$; IR: $v=1607,1564,1438$, $1385,1330,1304,1284,1255,1224,1155,1115,1082,975,811,760,732 \mathrm{~cm}^{-1}$; HRMS (ESI) $\mathrm{m} / \mathrm{z}$ calc. for $\mathrm{C}_{17} \mathrm{H}_{16} \mathrm{BNF}_{3}[\mathrm{M}+\mathrm{H}]^{+} 302.1328$, found 302.1334 .<smiles>COCCCc1ccc(P2C=Cc3ccccc3N2)cc1</smiles>

2-(4-(3-Methoxypropyl)phenyl)-2,1-borazanaphthalene (2d): obtained as an off white powder (133 mg, 96\%); mp $=76{ }^{\circ} \mathrm{C} ;{ }^{1} \mathrm{H}$ NMR (acetone- $d_{6}, 500.4 \mathrm{MHz}$ ): $\delta 9.69$ (br s, $1 \mathrm{H}$ ), 8.15 (d, $J=11.7 \mathrm{~Hz}, 1 \mathrm{H}), 7.97$ (d, $J=7.4 \mathrm{~Hz}, 2 \mathrm{H}), 7.69-7.64$ (m, $2 \mathrm{H}), 7.44$ (dd, $J=7.0,7.0$ $\mathrm{Hz}, 1 \mathrm{H}), 7.30-7.28(\mathrm{~m}, 3 \mathrm{H}), 7.17$ (dd, $J=7.6,7.5 \mathrm{~Hz}, 1 \mathrm{H}), 3.36(\mathrm{t}, J=5.8 \mathrm{~Hz}, 2 \mathrm{H}), 3.28$ (s, $3 \mathrm{H}), 2.70(\mathrm{t}, J=7.2 \mathrm{~Hz}, 2 \mathrm{H}), 1.87(\mathrm{dt}, J=7.2,5.8 \mathrm{~Hz}, 2 \mathrm{H}) \mathrm{ppm} ;{ }^{13} \mathrm{C}\left\{{ }^{1} \mathrm{H}\right\}$ NMR (acetone$\left.d_{6}, 125.8 \mathrm{MHz}\right): \delta 145.2,143.6,141.1,133.2,129.1,128.2,128.1,125.6,120.6,118.5,71.4$, 57.5, 32.0, $31.2 \mathrm{ppm} ;{ }^{11}$ B NMR (acetone, $128.4 \mathrm{MHz}$ ): $\delta 34.3 \mathrm{ppm}$; IR: $v=1605,1595,1562$, 1437, 1389, 1346, 1282, 1208, 1193, 1118, 1067, 977, 952, 942, 813, $801 \mathrm{~cm}^{-1}$; HRMS (ESI) $\mathrm{m} / \mathrm{z}$ calc. for $\mathrm{C}_{18} \mathrm{H}_{20} \mathrm{BNO}[\mathrm{M}]^{+} 277.1638$, found 277.1632.<smiles>CC(C)Cc1ccc(N2C=Cc3ccccc3N2)cc1</smiles>

2-(4-Isobutylphenyl)-2,1-borazanaphthalene (2e): obtained as a white powder (122 $\mathrm{mg}$, 94\%); mp $=109{ }^{\circ} \mathrm{C} ;{ }^{-1} \mathrm{H}$ NMR (acetone- $d_{6}, 500.4 \mathrm{MHz}$ ): $\delta 9.67$ (br s, $\left.1 \mathrm{H}\right), 8.15(\mathrm{~d}, J=11.5$ $\mathrm{Hz}, 1 \mathrm{H}), 7.96(\mathrm{~d}, J=7.9 \mathrm{~Hz}, 2 \mathrm{H}), 7.69-7.63(\mathrm{~m}, 2 \mathrm{H}), 7.44$ (ddd, $J=7.4,7.4,1.0 \mathrm{~Hz}, 1 \mathrm{H})$, 7.29 (dd, $J=11.9,1.0 \mathrm{~Hz}, 1 \mathrm{H}), 7.25(\mathrm{~d}, J=8.2 \mathrm{~Hz}, 2 \mathrm{H}), 7.17$ (dd, $J=7.2,7.3 \mathrm{~Hz}, 1 \mathrm{H}), 2.52$ $(\mathrm{d}, J=7.0 \mathrm{~Hz}, 2 \mathrm{H}), 1.91$ (sept, $J=6.7 \mathrm{~Hz}, 1 \mathrm{H}), 0.92(\mathrm{~d}, J=6.6 \mathrm{~Hz}, 6 \mathrm{H}) \mathrm{ppm} ;{ }^{13} \mathrm{C}\left\{{ }^{1} \mathrm{H}\right\}$ NMR (acetone- $d_{6}, 125.8 \mathrm{MHz}$ ): $\delta$ 145.2, 142.9, 141.1, 133.0, 129.1, 128.7, 128.2, 125.6, 120.6, 118.5, 45.1, 30.0, 21.8 ppm; ${ }^{11}$ B NMR (acetone, 128.4 MHz): $\delta 34.2 \mathrm{ppm}$; IR: $v=$ 2952, 2865, 1612, 1596, 1566, 1460, 1440, 1402, 1384, 1346, 1284, 1200, 1118, 978, 813, $791 \mathrm{~cm}^{-1}$; HRMS (ESI) $\mathrm{m} / \mathrm{z}$ calc. for $\mathrm{C}_{18} \mathrm{H}_{21} \mathrm{BN}[\mathrm{M}+\mathrm{H}]^{+} 262.1767$, found 262.1769 . 


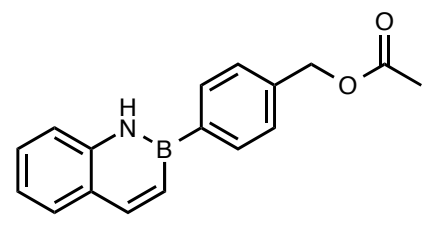

2-(4-(Acetoxymethyl)phenyl)-2,1-borazanaphthalene (2f): obtained as a white powder (104 $\mathrm{mg}, 75 \%$ ) using silicate $\mathbf{1 1 b} ; \mathrm{mp}=95{ }^{\circ} \mathrm{C} ;{ }^{1} \mathrm{H}$ NMR (acetone- $\left.d_{6}, 500.4 \mathrm{MHz}\right): \delta 9.79(\mathrm{br} \mathrm{s}, 1$ H), $8.18(\mathrm{~d}, J=11.6 \mathrm{~Hz}, 1 \mathrm{H}), 8.05(\mathrm{~d}, J=8.0 \mathrm{~Hz}, 2 \mathrm{H}), 7.70-7.64(\mathrm{~m}, 2 \mathrm{H}), 7.49-7.42$ (m, 3 H), 7.29 (dd, $J=11.5,2.1 \mathrm{~Hz}, 1 \mathrm{H}), 7.18$ (ddd, $J=7.9,7.1,1.1 \mathrm{~Hz}, 1 \mathrm{H}), 5.15(\mathrm{~s}, 2 \mathrm{H}), 2.07$ $(\mathrm{s}, 3 \mathrm{H}) \mathrm{ppm} ;{ }^{13} \mathrm{C}\left\{{ }^{1} \mathrm{H}\right\}$ NMR (acetone- $\left.d_{6}, 125.8 \mathrm{MHz}\right): \delta 170.0,145.4,141.0,137.7,133.2$, 129.1, 128.3 127.5, 125.6, 120.8, 118.6, 65.5, $19.9 \mathrm{ppm} ;{ }^{11} \mathrm{~B}$ NMR (acetone, $128.4 \mathrm{MHz}$ ): $\delta$ 34.0 ppm; IR: $v=1738,1609,1595,1563,1437,1377,1361,1226,1152,1117,1039,1018$, 988, 977, 939, $806 \mathrm{~cm}^{-1}$; HRMS (ESI) $\mathrm{m} / \mathrm{z}$ calc. for $\mathrm{C}_{17} \mathrm{H}_{16} \mathrm{BNNaO}_{2}[\mathrm{M}+\mathrm{Na}]^{+} 300.1172$, found 300.1169 .<smiles>CC(=O)OCCCc1ccc(B2C=Cc3ccccc3N2)cc1</smiles>

2-(4-(3-Acetoxypropyl)phenyl)-2,1-borazanaphthalene (2g): obtained as an off white powder $(141 \mathrm{mg}, 92 \%) ; \mathrm{mp}=71{ }^{\circ} \mathrm{C} ;{ }^{1} \mathrm{H}$ NMR (acetone- $\left.d_{6}, 500.4 \mathrm{MHz}\right): \delta 9.70$ (br s, $\left.1 \mathrm{H}\right)$, $8.15(\mathrm{~d}, J=8.6 \mathrm{~Hz}, 1 \mathrm{H}), 7.98(\mathrm{~d}, J=7.8 \mathrm{~Hz}, 2 \mathrm{H}), 7.69(\mathrm{~d}, J=8.1 \mathrm{~Hz}, 1 \mathrm{H}), 7.66(\mathrm{~d}, J=8.1$ $\mathrm{Hz}, 1 \mathrm{H}), 7.45$ (ddd, $J=8.1,6.7,1.3 \mathrm{~Hz}, 1 \mathrm{H}), 7.31$ (d, $J=7.8 \mathrm{~Hz}, 2 \mathrm{H}), 7.28$ (dd, $J=11.5$, $1.8 \mathrm{~Hz}, 1 \mathrm{H}), 7.17(\mathrm{ddd}, J=8.0,7.0,1.3 \mathrm{~Hz}, 1 \mathrm{H}), 4.07(\mathrm{t}, J=6.6 \mathrm{~Hz}, 2 \mathrm{H}), 2.74(\mathrm{t}, J=7.7$ $\mathrm{Hz}, 2 \mathrm{H}), 2.00(\mathrm{~s}, 3 \mathrm{H}), 2.00-1.95(\mathrm{~m}, 2 \mathrm{H}) \mathrm{ppm} ;{ }^{13} \mathrm{C}\left\{{ }^{1} \mathrm{H}\right\}$ NMR (acetone- $\left.d_{6}, 125.8 \mathrm{MHz}\right): \delta$ 170.1, 145.2, 142.9, 141.0, 133.2, 129.1, 128.2, 128.0, 125.6, 120.6, 118.5, 63.2, 31.8, 30.1, 19.9 ppm; ${ }^{11} \mathrm{~B}$ NMR (acetone, $128.4 \mathrm{MHz}$ ): $\delta 34.7 \mathrm{ppm}$; IR: $v=3354,1717,1610,1562$, 1444, 1403, 1388, 136, 1344, 1253, 1191, 1154, 1033, 1002, 979, $815 \mathrm{~cm}^{-1} ; \mathrm{HRMS}(\mathrm{ESI}) \mathrm{m} / \mathrm{z}$ calc. for $\mathrm{C}_{19} \mathrm{H}_{20} \mathrm{BNNaO}_{2}[\mathrm{M}+\mathrm{Na}]^{+} 328.1485$, found 328.1481 .<smiles>CC(=O)NCCCc1ccc(B2C=Cc3ccccc3N2)cc1</smiles>

2-(4-(3-Acetamidopropyl)phenyl)-2,1-borazanaphthalene (2h): obtained as an off white powder $(135 \mathrm{mg}, 89 \%) ; \mathrm{mp}=157{ }^{\circ} \mathrm{C} ;{ }^{1} \mathrm{H} \mathrm{NMR}\left(\mathrm{CDCl}_{3}, 500.4 \mathrm{MHz}\right): \delta 8.17($ br s, $1 \mathrm{H}), 8.13$ $(\mathrm{d}, J=11.3 \mathrm{~Hz}, 1 \mathrm{H}), 7.86(\mathrm{~d}, J=7.8 \mathrm{~Hz}, 2 \mathrm{H}), 7.68(\mathrm{~d}, J=8.3 \mathrm{~Hz}, 1 \mathrm{H}), 7.45(\mathrm{ddd}, J=7.9$, 7.2, $1.4 \mathrm{~Hz}, 1 \mathrm{H}), 7.35(\mathrm{~d}, J=8.5 \mathrm{~Hz}, 1 \mathrm{H}), 7.30(\mathrm{~d}, J=7.5 \mathrm{~Hz}, 2 \mathrm{H}), 7.27(\mathrm{dd}, J=11.6,2.1$ Hz, $1 \mathrm{H}), 7.20$ (ddd, $J=7.9,7.0,0.9 \mathrm{~Hz}, 1 \mathrm{H}), 5.49$ (br s, $1 \mathrm{H}), 3.33$ (td, $J=6.8,6.5 \mathrm{~Hz}, 2 \mathrm{H}$ ), $2.72(\mathrm{t}, J=7.7 \mathrm{~Hz}, 2 \mathrm{H}), 1.96(\mathrm{~s}, 3 \mathrm{H}), 1.90(\mathrm{tt}, J=7.7,6.8 \mathrm{~Hz}, 2 \mathrm{H}) \mathrm{ppm} ;{ }^{13} \mathrm{C}\left\{{ }^{1} \mathrm{H}\right\} \mathrm{NMR}$ $\left(\mathrm{CDCl}_{3}, 125.8 \mathrm{MHz}\right): \delta 170.0,145.4,143.0,140.1,133.0,132.9,129.4,128.3,125.6,120.9$, 118.1, 39.3, 33.3, 31.0, $23.3 \mathrm{ppm} ;{ }^{11} \mathrm{~B}$ NMR (acetone, $128.4 \mathrm{MHz}$ ): $\delta 34.1 \mathrm{ppm}$; IR: $v=1633$, 1606, 1595, 1551, 1435, 1404, 1366, 1344, 1301, 1281, 1200, 1182, 810, 757, 745, $719 \mathrm{~cm}^{-1}$; HRMS (ESI) m/z calc. for $\mathrm{C}_{19} \mathrm{H}_{22} \mathrm{BN}_{2} \mathrm{O}[\mathrm{M}+\mathrm{H}]^{+} 305.1825$, found 305.1816 . 


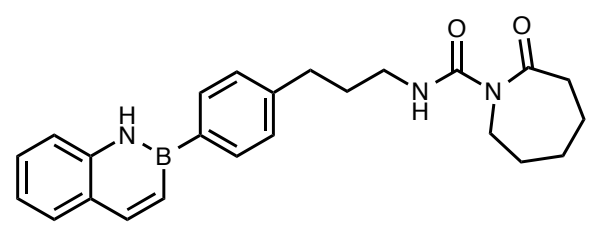

2-(4-(3-(2-Oxoazepane-1-carboxamido)propyl)phenyl)-2,1-borazanaphthalene

(2i): obtained as an off white powder (192 mg, 96\%); mp $=133{ }^{\circ} \mathrm{C} ;{ }^{1} \mathrm{H}$ NMR (DMSO- $d_{6}, 500.4$ MHz): $\delta 10.34$ (br s, $1 \mathrm{H}), 9.22$ (br s, $1 \mathrm{H}), 8.11$ (d, $J=11.4 \mathrm{~Hz}, 1 \mathrm{H}), 7.99$ (d, $J=8.0 \mathrm{~Hz}, 2$ H), $7.71(\mathrm{~d}, J=8.3 \mathrm{~Hz}, 1 \mathrm{H}), 7.66(\mathrm{~d}, J=8.0 \mathrm{~Hz}, 1 \mathrm{H}), 7.44(\mathrm{t}, J=7.6 \mathrm{~Hz}, 1 \mathrm{H}), 7.29(\mathrm{~d}, J=$ $7.1 \mathrm{~Hz}, 2 \mathrm{H}), 7.22(\mathrm{~d}, J=11.1 \mathrm{~Hz}, 1 \mathrm{H}), 7.14(\mathrm{t}, J=7.1 \mathrm{~Hz}, 1 \mathrm{H}), 3.90(\mathrm{~d}, J=2.1 \mathrm{~Hz}, 2 \mathrm{H})$, 3.18-3.24 (m, $2 \mathrm{H}), 2.71-2.68(\mathrm{~m}, 2 \mathrm{H}), 2.62(\mathrm{t}, J=4.3 \mathrm{~Hz}, 2 \mathrm{H}), 1.85-1.80$ (m, $2 \mathrm{H}), 1.68-$ $1.62(\mathrm{~m}, 4 \mathrm{H}), 1.58-1.53(\mathrm{~m}, 2 \mathrm{H}) \mathrm{ppm} ;{ }^{13} \mathrm{C}\left\{{ }^{1} \mathrm{H}\right\}$ NMR (DMSO-d, $\left.125.8 \mathrm{MHz}\right): \delta 179.4$, 154.6, 145.5, 143.3, 141.4, 133.9, 129.4, 128.6, 128.3, 125.5, 120.9, 119.0, 43.2, 39.9, 39.3, 33.0, 30.9, 28.7, 28.3, $23.4 \mathrm{ppm} ;{ }^{11} \mathrm{~B}$ NMR (acetone, $128.4 \mathrm{MHz}$ ): $\delta 33.9 \mathrm{ppm}$; IR: $v=3390$, $3258,1686,1651,1614,1568,1524,1451,1440,1396,1351,1289,1205,1174,1159,814$, 776, $748 \mathrm{~cm}^{-1}$; HRMS (ESI) m/z calc. for $\mathrm{C}_{24} \mathrm{H}_{29} \mathrm{BN}_{3} \mathrm{O}_{2}[\mathrm{M}+\mathrm{H}]^{+}$402.2344, found 402.2353.

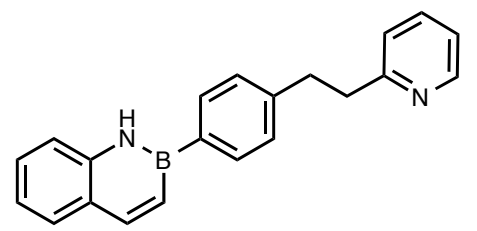

2-(4-(2-Pyridin-2-yl)ethyl)phenyl)-2,1-borazanaphthalene (2j): obtained as an off white powder $(125 \mathrm{mg}, 81 \%) ; \mathrm{mp}=117^{\circ} \mathrm{C} ;{ }^{1} \mathrm{H}$ NMR (acetone- $\left.d_{6}, 500.4 \mathrm{MHz}\right): \delta 9.73$ (br s, $\left.1 \mathrm{H}\right)$, $8.52(\mathrm{~d}, J=4.9 \mathrm{~Hz}, 1 \mathrm{H}), 8.16(\mathrm{~d}, J=11.2 \mathrm{~Hz}, 1 \mathrm{H}), 7.95$ (d, $J=7.3 \mathrm{~Hz}, 2 \mathrm{H}), 7.69-7.60$ (m, $3 \mathrm{H}), 7.44$ (ddd, $J=7.9,7.4,1.4 \mathrm{~Hz}, 1 \mathrm{H}), 7.32-7.26(\mathrm{~m}, 3 \mathrm{H}), 7.21(\mathrm{~d}, J=8.0 \mathrm{~Hz}, 1 \mathrm{H}) 7.19$ $7.12(\mathrm{~m}, 2 \mathrm{H}), 3.13-3.08(\mathrm{~m}, 4 \mathrm{H}) \mathrm{ppm} ;{ }^{13} \mathrm{C}\left\{{ }^{1} \mathrm{H}\right\}$ NMR (acetone- $\left.d_{6}, 125.8 \mathrm{MHz}\right): \delta 161.1$, $149.1,145.2$, 143.3, 141.1, 136.0, 133.2, 129.1, 128.2, 128.1, 125.6, 122.7, 121.0, 120.6, 118.5, 39.6, $35.5 \mathrm{ppm} ;{ }^{11} \mathrm{~B}$ NMR (acetone, $128.4 \mathrm{MHz}$ ): $\delta 35.1 \mathrm{ppm}$; IR: $v=3372,1605$, 1593, 1565, 1471, 1431, 1404, 1386, 1347, 1282, 1229, 1199, 1139, 977, 808, 756, $745 \mathrm{~cm}^{-1}$; HRMS (ESI) $\mathrm{m} / \mathrm{z}$ calc. for $\mathrm{C}_{21} \mathrm{H}_{20} \mathrm{BN}_{2}[\mathrm{M}+\mathrm{H}]^{+} 311.1720$, found 311.1724 .<smiles>C1=Cc2ccccc2NB1c1ccc(CCCN2CCNCC2)cc1</smiles>

2-(4-(3-(Piperazin-1-yl)propyl)phenyl)-2,1-borazanaphthalene (2k): obtained as a colorless oil (78 mg, 47\%) using silicate 11c; ${ }^{1} \mathrm{H}$ NMR (acetone- $d_{6}, 500.4 \mathrm{MHz}$ ): $\delta 8.16$ (br s, $1 \mathrm{H}), 8.13(\mathrm{~d}, J=11.5 \mathrm{~Hz}, 1 \mathrm{H}), 7.66(\mathrm{~d}, J=7.7 \mathrm{~Hz}, 2 \mathrm{H}), 7.66(\mathrm{dd}, J=8.0,1.1 \mathrm{~Hz}, 1 \mathrm{H}), 7.44$ (ddd, $J=8.3,6.9,1.2 \mathrm{~Hz}, 1 \mathrm{H}), 7.35-7.30(\mathrm{~m}, 3 \mathrm{H}), 7.29$ (d, $J=12 \mathrm{H}, 1 \mathrm{H}), 7.20(\mathrm{t}, J=7.4$ $\mathrm{Hz}, 1 \mathrm{H}), 2.96-2.90(\mathrm{~m}, 4 \mathrm{H}), 2.71(\mathrm{t}, J=7.7 \mathrm{~Hz}, 2 \mathrm{H}), 2.51-2.37(\mathrm{~m}, 6 \mathrm{H}), 2.32-2.20$ (br s, 1 $\mathrm{H}), 1.89(\mathrm{tt}, J=8.5,6.7 \mathrm{~Hz}, 2 \mathrm{H}) \mathrm{ppm} ;{ }^{13} \mathrm{C}\left\{{ }^{1} \mathrm{H}\right\}$ NMR (acetone- $d_{6}, 125.8 \mathrm{MHz}$ ): $\delta 145.3$, $143.9,140.2,132.8,129.4,128.4,128.3,125.6,120.9,118.1,58.6,54.5,46.0,33.7,28.2$ ppm; ${ }^{11} \mathrm{~B}$ NMR (acetone, $128.4 \mathrm{MHz}$ ): $\delta 34.2 \mathrm{ppm}$; IR: $v=2939,2806,1612,1595,1563$, $1437,1345,1283,1233,1217,1199,1188,1142,1116,979,801 \mathrm{~cm}^{-1}$; HRMS (ESI) m/z calc. for $\mathrm{C}_{21} \mathrm{H}_{27} \mathrm{BN}_{3}[\mathrm{M}+\mathrm{H}]^{+}$332.2298, found 332.2307. 


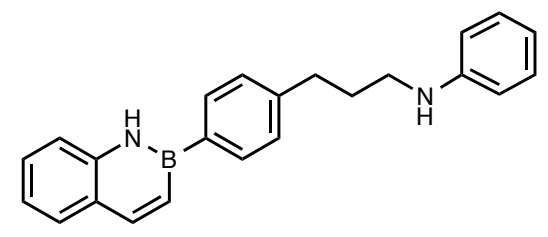

2-(4-(3-(Phenylamino)propyl)phenyl)-2,1-borazanaphthalene (2I): obtained as a white powder (154 mg, 91\%); mp $=110{ }^{\circ} \mathrm{C} ;{ }^{1} \mathrm{H}$ NMR (acetone- $d_{6}, 500.4 \mathrm{MHz}$ ): $\delta 9.69$ (br s, $1 \mathrm{H}$ ), $8.16(\mathrm{~d}, J=11.0 \mathrm{~Hz}, 1 \mathrm{H}), 7.98$ (d, $J=7.8 \mathrm{~Hz}, 2 \mathrm{H}), 7.67$ (t, $J=8.6 \mathrm{~Hz}, 2 \mathrm{H}), 7.44$ (ddd, $J=$ 8.2, 7.2, $1.5 \mathrm{~Hz}, 1 \mathrm{H}), 7.34-7.26(\mathrm{~m}, 3 \mathrm{H}), 7.17$ (ddd, $J=8.0,6.8,1.2 \mathrm{~Hz}, 1 \mathrm{H}), 7.10-7.05$ (m, $2 \mathrm{H}), 6.61(\mathrm{dd}, J=8.2,1.1 \mathrm{~Hz}, 2 \mathrm{H}), 6.56(\mathrm{t}, J=7.3 \mathrm{~Hz}, 1 \mathrm{H}), 4.85$ (br s, $1 \mathrm{H}), 3.18-3.12(\mathrm{~m}$, $2 \mathrm{H}), 2.78(\mathrm{t}, J=7.7 \mathrm{~Hz}, 2 \mathrm{H}), 2.00-1.94(\mathrm{~m}, 2 \mathrm{H}) \mathrm{ppm} ;{ }^{13} \mathrm{C}\left\{{ }^{1} \mathrm{H}\right\}$ NMR (acetone- $d_{6}, 125.8$ $\mathrm{MHz}): \delta 149.1,145.2,143.6,141.1,133.2,129.1,128.8,128.2,128.1,125.6,120.6,118.5$, 115.9, 112.2, 42.8, 33.2, 30.9 ppm; ${ }^{11} \mathrm{~B}$ NMR (acetone, $128.4 \mathrm{MHz}$ ): $\delta 34.4 \mathrm{ppm}$; IR: $v=$ 3350, 1596, 1561, 1503, 1480, 1438, 1405, 1311, 1282, 1236, 1183, 1163, 1148, 1118, 810, 757, $743 \mathrm{~cm}^{-1}$; HRMS (ESI) m/z calc. for $\mathrm{C}_{23} \mathrm{H}_{24} \mathrm{BN}_{2}[\mathrm{M}+\mathrm{H}]^{+} 339.2033$, found 339.2032.

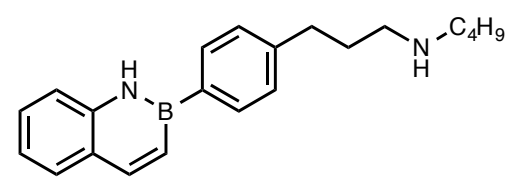

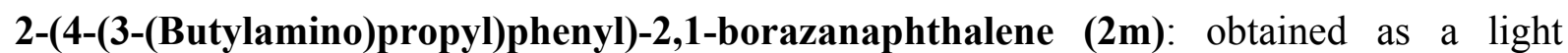
brown powder (152 mg, 95\%) using silicate 11d; $\mathrm{mp}=91{ }^{\circ} \mathrm{C} ;{ }^{1} \mathrm{H} \mathrm{NMR}\left(\mathrm{CDCl}_{3}, 500.4 \mathrm{MHz}\right)$ : $\delta$ 8.18-8.12 (m, $2 \mathrm{H}), 7.88(\mathrm{~d}, J=7.8 \mathrm{~Hz}, 2 \mathrm{H}), 7.67$ (d, $J=7.8 \mathrm{~Hz}, 1 \mathrm{H}), 7.46$ (ddd, $J=8.6$, 6.4, $1.4 \mathrm{~Hz}, 1 \mathrm{H}), 7.36-7.27$ (m, $4 \mathrm{H}), 7.21$ (ddd, $J=8.1,7.5,1.3 \mathrm{~Hz}, 1 \mathrm{H}), 2.77-2.69$ (m, 4 H), 2.64 (t, $J=7.2 \mathrm{~Hz}, 2 \mathrm{H}), 2.19$ (br s, $1 \mathrm{H}), 1.90(\mathrm{tt}, J=7.8,7.2 \mathrm{~Hz}, 2 \mathrm{H}), 1.51$ (tt, $J=8.0$, $7.2 \mathrm{~Hz}, 2 \mathrm{H}), 1.40-1.33(\mathrm{~m}, 2 \mathrm{H}), 0.95$ (t, $J=7.5 \mathrm{~Hz}, 3 \mathrm{H}) \mathrm{ppm} ;{ }^{13} \mathrm{C}\left\{{ }^{1} \mathrm{H}\right\} \mathrm{NMR}\left(\mathrm{CDCl}_{3}\right.$, $125.8 \mathrm{MHz}): \delta 145.3,143.8,140.2,132.8,129.4,128.3,128.2,125.6,120.9,118.1,49.7$, 49.5, 33.7, 32.2, 31.5, 20.4, $13.9 \mathrm{ppm} ;{ }^{11} \mathrm{~B}$ NMR (acetone, $128.4 \mathrm{MHz}$ ): $\delta 34.6 \mathrm{ppm}$; IR: $v=$ 2988, 1608, 1597, 1563, 1437, 1405, 1345, 1282, 1234, 1211, 1183, 1103, 977, 881, 796, 758, $733 \mathrm{~cm}^{-1}$; HRMS (ESI) m/z calc. for $\mathrm{C}_{21} \mathrm{H}_{28} \mathrm{BN}_{2}[\mathrm{M}+\mathrm{H}]^{+} 319.2346$, found 319.2351.<smiles>NCCCc1ccc(B2C=Cc3ccccc3N2)cc1</smiles>

2-(4-(3-Aminopropyl)phenyl)-2,1-borazanaphthalene (2n): obtained as a white powder (89 $\mathrm{mg}, 68 \%) ; \mathrm{mp}=121{ }^{\circ} \mathrm{C} ;{ }^{1} \mathrm{H}$ NMR (acetone- $\left.d_{6}, 500.4 \mathrm{MHz}\right): \delta 9.71(\mathrm{br} \mathrm{s}, 1 \mathrm{H}), 8.15(\mathrm{~d}, J=$ $11.6 \mathrm{~Hz}, 1 \mathrm{H}), 7.96(\mathrm{~d}, J=7.9 \mathrm{~Hz}, 2 \mathrm{H}), 7.69-7.65(\mathrm{~m}, 2 \mathrm{H}), 7.43$ (ddd, $J=8.5,6.8,1.4 \mathrm{~Hz}, 1$ H), 7.31-7.26 (m, $3 \mathrm{H}), 7.16(\mathrm{ddd}, J=8.1,6.8,1.4 \mathrm{~Hz}, 1 \mathrm{H}), 3.21(\mathrm{t}, J=6.8 \mathrm{~Hz}, 2 \mathrm{H}), 2.72(\mathrm{t}$, $J=7.1 \mathrm{~Hz}, 2 \mathrm{H}), 1.95-1.71(\mathrm{~m}, 4 \mathrm{H}) \mathrm{ppm} ;{ }^{13} \mathrm{C}\left\{{ }^{1} \mathrm{H}\right\}$ NMR (acetone- $\left.d_{6}, 125.8 \mathrm{MHz}\right): \delta 145.1$, $144.1,141.1,133.1,129.1,128.2,128.1,125.6,120.5,118.5,50.2,33.4,32.5$ ppm; ${ }^{11} \mathrm{~B}$ NMR (acetone, 128.4 MHz): $\delta 34.2$ ppm; IR: $v=2941,1651,1609,1575,1455,1417,1349,1287$, $1208,1193,978,941,893,813,790,759 \mathrm{~cm}^{-1}$; HRMS (ESI) m/z calc. for $\mathrm{C}_{17} \mathrm{H}_{20} \mathrm{BN}_{2}[\mathrm{M}+$ $\mathrm{H}]^{+} 263.1720$, found 263.1711 . 


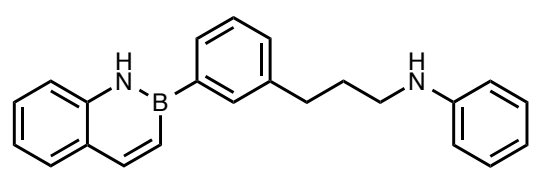

2-(3-(3-(Phenylamino)propyl)phenyl)-2,1-borazanaphthalene (3a): obtained as a white powder $(153 \mathrm{mg}, 90 \%) ; \mathrm{mp}=75{ }^{\circ} \mathrm{C} ;{ }^{1} \mathrm{H} \mathrm{NMR}\left(\mathrm{CDCl}_{3}, 500.4 \mathrm{MHz}\right): \delta 8.18(\mathrm{~d}, J=11.3 \mathrm{~Hz}, 1$ H), $8.14($ br s, $1 \mathrm{H}), 7.81(\mathrm{dt}, J=7.2,1.2 \mathrm{~Hz}, 1 \mathrm{H}), 7.78(\mathrm{~s}, 1 \mathrm{H}), 7.70(\mathrm{dd}, J=7.9,1.3 \mathrm{~Hz}, 1$ H), 7.51-7.44 (m, $2 \mathrm{H}), 7.38-7.30(\mathrm{~m}, 3 \mathrm{H}), 7.27-7.20(\mathrm{~m}, 3 \mathrm{H}), 6.75(\mathrm{tt}, J=7.2,1.0 \mathrm{~Hz}, 1$ H), $6.65(\mathrm{dd}, J=8.4,0.8 \mathrm{~Hz}, 2 \mathrm{H}), 3.67(\mathrm{~s}, 1 \mathrm{H}), 3.24(\mathrm{t}, J=7.1 \mathrm{~Hz}, 2 \mathrm{H}), 2.87(\mathrm{t}, J=7.6 \mathrm{~Hz}$, $2 \mathrm{H}), 2.06(\mathrm{tt}, J=7.6,7.1 \mathrm{~Hz}, 2 \mathrm{H}) \mathrm{ppm} ;{ }^{13} \mathrm{C}\left\{{ }^{1} \mathrm{H}\right\} \mathrm{NMR}\left(\mathrm{CDCl}_{3}, 125.8 \mathrm{MHz}\right): \delta 148.3,145.5$, 141.3, 140.1, 132.8, 130.4, 129.7, 129.4, 129.2, 128.3 [× 2], 125.7, 121.0, 118.2, 117.2, 112.7, 43.4, 33.5, $31.2 \mathrm{ppm} ;{ }^{11} \mathrm{~B}$ NMR (acetone, $128.4 \mathrm{MHz}$ ): $\delta 34.5 \mathrm{ppm}$; IR: $v=3325,1599,1579$, $1561,1507,135,1389,1346,1334,1283,1262,1245,1210,1184,1153,795,762 \mathrm{~cm}^{-1}$; HRMS (ESI) $\mathrm{m} / \mathrm{z}$ calc. for $\mathrm{C}_{23} \mathrm{H}_{24} \mathrm{BN}_{2}[\mathrm{M}+\mathrm{H}]^{+} 339.2033$, found 339.2036.

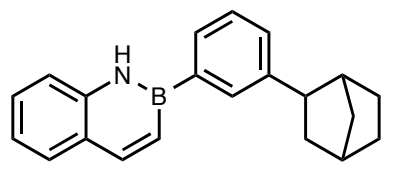

2-(3-(exo-Bicyclo[2.2.1]heptan-2-yl)phenyl)-2,1-borazanaphthalene (3b): obtained as a light brown powder $(143 \mathrm{mg}, 96 \%) ; \mathrm{mp}=115{ }^{\circ} \mathrm{C} ;{ }^{1} \mathrm{H} \mathrm{NMR}\left(\mathrm{CDCl}_{3}, 500.4 \mathrm{MHz}\right): \delta 8.13(\mathrm{~d}, J$ $=11.7 \mathrm{~Hz}, 2 \mathrm{H}), 7.75(\mathrm{~s}, 1 \mathrm{H}), 7.70(\mathrm{~d}, J=7.5 \mathrm{~Hz}, 1 \mathrm{H}), 7.65(\mathrm{~d}, J=7.7 \mathrm{~Hz}, 1 \mathrm{H}), 7.43(\mathrm{ddd}, J$ $=8.6,7.2,1.4 \mathrm{~Hz}, 1 \mathrm{H}), 7.39(\mathrm{t}, J=7.4 \mathrm{~Hz}, 1 \mathrm{H}), 7.35-7.30(\mathrm{~m}, 2 \mathrm{H}), 7.27(\mathrm{dd}, J=11.6,2.2$ $\mathrm{Hz}, 1 \mathrm{H}), 7.18(\mathrm{ddd}, J=7.9,7.0,1.1 \mathrm{~Hz}, 1 \mathrm{H}), 2.84(\mathrm{dd}, J=8.3,5.8 \mathrm{~Hz}, 1 \mathrm{H}), 2.45-2.43(\mathrm{~m}, 1$ H), 2.41-2.37 (m, $1 \mathrm{H}), 1.86-1.80(\mathrm{~m}, 1 \mathrm{H}), 1.79-1.73(\mathrm{~m}, 1 \mathrm{H}), 1.67-1.55(\mathrm{~m}, 3 \mathrm{H}), 1.44$ $1.38(\mathrm{~m}, 1 \mathrm{H}), 1.34-1.28(\mathrm{~m}, 1 \mathrm{H}), 1.26-1.20(\mathrm{~m}, 1 \mathrm{H}) \mathrm{ppm} ;{ }^{13} \mathrm{C}\left\{{ }^{1} \mathrm{H}\right\} \mathrm{NMR}\left(\mathrm{CDCl}_{3}, 125.8\right.$ $\mathrm{MHz}): \delta 147.2,145.4,140.2,131.8,129.8,129.5,128.4,128.3,128.2,125.8,121.0,118.3$, 47.6, 43.1, 39.2, 36.9, 36.2, 30.7, 29.0 ppm; ${ }^{11} \mathrm{~B}$ NMR (acetone, $128.4 \mathrm{MHz}$ ): $\delta 34.5 \mathrm{ppm}$; IR: $v=2948,2866,1613,1595,1561,1435,1349,1288,1267,1244,1210,1188,1119,818$, 791, $756 \mathrm{~cm}^{-1}$; HRMS (ESI) m/z calc. for $\mathrm{C}_{21} \mathrm{H}_{21} \mathrm{BN}[\mathrm{M}-\mathrm{H}]^{-} 298.1767$, found 298.1756.<smiles>CC(=O)NCCCc1cccc(B2C=Cc3ccccc3N2)c1</smiles>

2-(3-(3-Acetamidopropyl)phenyl)-2,1-borazanaphthalene (3c): obtained as an off white powder $(146 \mathrm{mg}, 96 \%) ; \mathrm{mp}=78{ }^{\circ} \mathrm{C} ;{ }^{1} \mathrm{H} \mathrm{NMR}\left(\mathrm{CDCl}_{3}, 500.4 \mathrm{MHz}\right): \delta 8.49$ (br s, $\left.1 \mathrm{H}\right), 8.11$ $(\mathrm{d}, J=11.4 \mathrm{~Hz}, 1 \mathrm{H}), 7.76-7.71(\mathrm{~m}, 2 \mathrm{H}), 7.63(\mathrm{~d}, J=8.0 \mathrm{~Hz}, 1 \mathrm{H}), 7.44-7.41(\mathrm{~m}, 2 \mathrm{H}), 7.37$ $(\mathrm{t}, J=7.5 \mathrm{~Hz}, 1 \mathrm{H}), 7.26-7.21(\mathrm{~m}, 2 \mathrm{H}), 7.19-7.15(\mathrm{~m}, 1 \mathrm{H}), 5.65(\mathrm{br} \mathrm{s}, 1 \mathrm{H}), 3.30(\mathrm{td}, J=6.6$, $6.7 \mathrm{~Hz}, 2 \mathrm{H}), 2.70(\mathrm{t}, J=7.4 \mathrm{~Hz}, 2 \mathrm{H}), 1.94(\mathrm{~s}, 3 \mathrm{H}), 1.87(\mathrm{tt}, J=7.7,7.1 \mathrm{~Hz}, 2 \mathrm{H}) \mathrm{ppm} ;{ }^{13} \mathrm{C}$ $\left\{{ }^{1} \mathrm{H}\right\}$ NMR $\left(\mathrm{CDCl}_{3}, 125.8 \mathrm{MHz}\right): \delta 170.2,145.4,140.8,140.4,132.8,130.6,129.6,129.3$, 128.3, 128.2, 125.6, 120.9, 118.4, 39.3, 33.1, 31.0, 23.3 ppm; ${ }^{11} \mathrm{~B}$ NMR (acetone, 128.4 MHz): $\delta 34.7$ ppm; IR: $v=3321,1649,1613,1595,1562,1436,1412,1386,1366,1347$, $1285,1242,1207,1181,810,785,759 \mathrm{~cm}^{-1}$; HRMS (ESI) m/z calc. for $\mathrm{C}_{19} \mathrm{H}_{21} \mathrm{BN}_{2} \mathrm{NaO}[\mathrm{M}+$ $\mathrm{Na}]^{+} 327.1645$, found 327.1635 . 


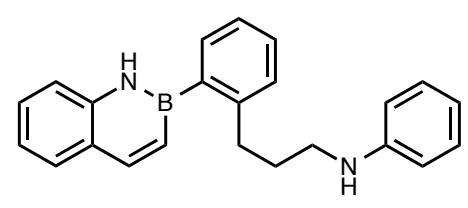

2-(2-(3-(Phenylamino)propyl)phenyl)-2,1-borazanaphthalene (4a): obtained as a light yellow oil $(158 \mathrm{mg}, 93 \%) ;{ }^{1} \mathrm{H} \mathrm{NMR}\left(\mathrm{CDCl}_{3}, 500.4 \mathrm{MHz}\right): \delta 8.08(\mathrm{~d}, J=11.7 \mathrm{~Hz}, 1 \mathrm{H}), 7.92$ (br s, $1 \mathrm{H}), 7.66(\mathrm{~d}, J=8.3 \mathrm{~Hz}, 1 \mathrm{H}), 7.48-7.44(\mathrm{~m}, 1 \mathrm{H}), 7.40$ (ddd, $J=8.2,7.1,1.5 \mathrm{~Hz}, 1 \mathrm{H})$, 7.37-7.32 (m, $1 \mathrm{H}), 7.28-7.23(\mathrm{~m}, 2 \mathrm{H}), 7.19$ (ddd, $J=7.9,7.0,1.1 \mathrm{~Hz}, 1 \mathrm{H}), 7.15(\mathrm{~d}, J=8.3$ $\mathrm{Hz}, 1 \mathrm{H}), 7.09-7.02(\mathrm{~m}, 3 \mathrm{H}), 6.64(\mathrm{tt}, J=7.3,1.1 \mathrm{~Hz}, 1 \mathrm{H}), 6.38(\mathrm{dd}, J=8.6,1.1 \mathrm{~Hz}, 2 \mathrm{H})$, 3.37 (br s, $1 \mathrm{H}), 3.03(\mathrm{t}, J=6.8 \mathrm{~Hz}, 2 \mathrm{H}), 2.90(\mathrm{t}, J=7.6 \mathrm{~Hz}, 2 \mathrm{H}), 1.85(\mathrm{tt}, J=7.6,6.8 \mathrm{~Hz}, 2$ H) ppm; ${ }^{13} \mathrm{C}\left\{{ }^{1} \mathrm{H}\right\}$ NMR $\left(\mathrm{CDCl}_{3}, 125.8 \mathrm{MHz}\right): \delta 148.2,144.9,140.0,133.0,129.4,129.1$, $128.7,128.6,128.4[\times 2], 125.5,125.3,121.2,118.3,117.2,112.7,43.4,33.6,31.9 \mathrm{ppm} ;{ }^{11} \mathrm{~B}$ NMR (acetone, $128.4 \mathrm{MHz}$ ): $\delta 36.1 \mathrm{ppm}$; IR: $v=3331,1614,1597,1559,1504,1430,1345$, 1320, 1280, 1262, 1235, 1203, 1179, 978, 907, 811, $746 \mathrm{~cm}^{-1}$; HRMS (ESI) m/z calc. for $\mathrm{C}_{23} \mathrm{H}_{24} \mathrm{BN}_{2}[\mathrm{M}+\mathrm{H}]^{+}$339.2033, found 339.2034.

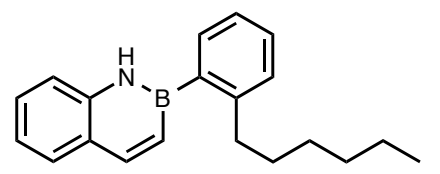

2-(2-Hexylphenyl)-2,1-borazanaphthalene (4b): obtained as a colorless oil (140 $\mathrm{mg}, 97 \%)$; ${ }^{1} \mathrm{H} \mathrm{NMR}\left(\mathrm{CDCl}_{3}, 500.4 \mathrm{MHz}\right): \delta 8.09(\mathrm{~d}, J=11.4 \mathrm{~Hz}, 1 \mathrm{H}), 7.93($ br s, $1 \mathrm{H}), 7.67$ (d, $J=8.3$ $\mathrm{Hz}, 1 \mathrm{H}), 7.47$ (dd, $J=7.3,1.3 \mathrm{~Hz}, 1 \mathrm{H}), 7.43$ (ddd, $J=8.4,6.8,1.2 \mathrm{~Hz}, 1 \mathrm{H}), 7.33$ (ddd, $J=$ 7.6, 7.4, 1.3 Hz, $1 \mathrm{H}), 7.29-7.16(\mathrm{~m}, 4 \mathrm{H}), 7.07(\mathrm{dd}, J=11.4,2.1 \mathrm{~Hz}, 1 \mathrm{H}), 2.77(\mathrm{t}, J=8.3 \mathrm{~Hz}$, $2 \mathrm{H}), 1.60-1.51(\mathrm{~m}, 2 \mathrm{H}), 1.31-1.17(\mathrm{~m}, 6 \mathrm{H}), 0.8(\mathrm{t}, J=6.8 \mathrm{~Hz}, 3 \mathrm{H}) \mathrm{ppm} ;{ }^{13} \mathrm{C}\left\{{ }^{1} \mathrm{H}\right\} \mathrm{NMR}$ $\left(\mathrm{CDCl}_{3}, 125.8 \mathrm{MHz}\right): \delta 146.3,144.7,140.1,133.0,129.5,128.7,128.5,128.3,125.4,125.3$, $121.1,118.2,36.2,32.7,31.7,29.3,22.6,14.1 \mathrm{ppm} ;{ }^{11} \mathrm{~B}$ NMR (acetone, $128.4 \mathrm{MHz}$ ): $\delta 36.3$ ppm; IR: $v=2952,2924,2854,1614,1596,1559,1430,1383,1345,1278,1232,1213,1203$, $1114,978,809 \mathrm{~cm}^{-1}$; HRMS (ESI) $\mathrm{m} / \mathrm{z}$ calc. for $\mathrm{C}_{20} \mathrm{H}_{23} \mathrm{BN}[\mathrm{M}-\mathrm{H}]^{-}$288.1924, found 288.1942 .<smiles>CC(=O)OCCCc1ccccc1B1C=Cc2ccccc2N1</smiles>

2-(2-(3-Acetoxypropyl)phenyl)-2,1-borazanaphthalene (4c): obtained as a colorless oil $(142 \mathrm{mg}, 93 \%) ;{ }^{1} \mathrm{H}$ NMR $\left(\mathrm{CDCl}_{3}, 500.4 \mathrm{MHz}\right): \delta 8.10(\mathrm{~d}, J=11.2 \mathrm{~Hz}, 1 \mathrm{H}), 7.80(\mathrm{br} \mathrm{s}, 1 \mathrm{H})$, $7.68(\mathrm{~d}, J=8.0 \mathrm{~Hz}, 1 \mathrm{H}), 7.50-7.41(\mathrm{~m}, 2 \mathrm{H}), 7.37-7.30(\mathrm{~m}, 2 \mathrm{H}), 7.28-7.24(\mathrm{~m}, 2 \mathrm{H}), 7.22$ (ddd, $J=8.0,7.0,1.2 \mathrm{~Hz}, 1 \mathrm{H}), 7.06(\mathrm{dd}, J=11.6,1.8 \mathrm{~Hz}, 1 \mathrm{H}), 3.99(\mathrm{t}, J=6.6 \mathrm{~Hz}, 2 \mathrm{H}), 2.87$ $(\mathrm{t}, J=7.8 \mathrm{~Hz}, 2 \mathrm{H}), 1.91-1.85(\mathrm{~m}, 2 \mathrm{H}), 1.84(\mathrm{~s}, 3 \mathrm{H}) \mathrm{ppm} ;{ }^{13} \mathrm{C}\left\{{ }^{1} \mathrm{H}\right\} \mathrm{NMR}\left(\mathrm{CDCl}_{3}, 125.8\right.$ $\mathrm{MHz}): \delta 171.1,144.8,144.4,140.2,133.0,129.4,128.6,128.5,128.4,125.6,125.3,121.2$, 118.3, 63.8, 32.3, 31.2, 20.7 ppm; ${ }^{11} \mathrm{~B}$ NMR (acetone, $128.4 \mathrm{MHz}$ ): $\delta 36.1 \mathrm{ppm}$; IR: $v=1723$, $1614,1596,1560,1432,1286,1365,1347,1236,1031,979,908,812,753,729,662 \mathrm{~cm}^{-1}$; HRMS (ESI) $\mathrm{m} / \mathrm{z}$ calc. for $\mathrm{C}_{19} \mathrm{H}_{21} \mathrm{BNO}_{2}[\mathrm{M}+\mathrm{H}]^{+}$306.1665, found 306.1666. 


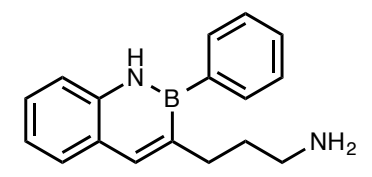

3-(3-Aminopropyl)-2-phenyl-2,1-borazanaphthalene (5a): obtained as a brown oil (124 mg, 95\%); ${ }^{1} \mathrm{H}$ NMR $\left(\mathrm{CDCl}_{3}, 500.4 \mathrm{MHz}\right): \delta 7.95$ (br s, $\left.1 \mathrm{H}\right), 7.86(\mathrm{~s}, 1 \mathrm{H}), 7.70-7.65$ (m, 2 H), $7.63(\mathrm{dd}, J=7.8,0.9 \mathrm{~Hz}, 1 \mathrm{H}), 7.48-7.37(\mathrm{~m}, 4 \mathrm{H}), 7.27(\mathrm{~d}, J=7.4 \mathrm{~Hz}, 1 \mathrm{H}), 7.20(\mathrm{t}, J=$ $7.5 \mathrm{~Hz}, 1 \mathrm{H}), 2.78(\mathrm{t}, J=7.6 \mathrm{~Hz}, 2 \mathrm{H}), 2.68(\mathrm{t}, J=7.6 \mathrm{~Hz}, 2 \mathrm{H}), 1.72(\mathrm{br} \mathrm{s}, 2 \mathrm{H}), 1.66(\mathrm{tt}, J=$ $7.6 \mathrm{~Hz}, 2 \mathrm{H}) \mathrm{ppm} ;{ }^{13} \mathrm{C}\left\{{ }^{1} \mathrm{H}\right\} \mathrm{NMR}\left(\mathrm{CDCl}_{3}, 125.8 \mathrm{MHz}\right): \delta 141.9,139.0,132.3,128.7,128.4$, $127.9,127.4,125.3,121.1,117.6,41.8,34.9,32.4 \mathrm{ppm} ;{ }^{11} \mathrm{~B}$ NMR (acetone, $128.4 \mathrm{MHz}$ ): $\delta$ 36.1 ppm; IR: $v=2926,2854,1661,1614,1597,1567,1453,1427,1372,1347,1285,1240$, $1208,945,909,751 \mathrm{~cm}^{-1}$; HRMS (ESI) $\mathrm{m} / \mathrm{z}$ calc. for $\mathrm{C}_{17} \mathrm{H}_{20} \mathrm{BN}_{2}[\mathrm{M}+\mathrm{H}]^{+} 263.1720$, found 263.1724 .

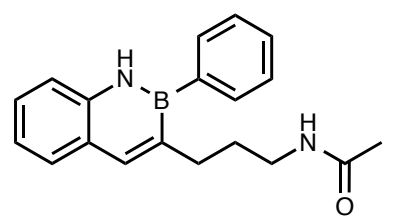

3-(3-Acetamidopropyl)-2-phenyl-2,1-borazanaphthalene (5b): obtained as an off white solid $(146 \mathrm{mg}, 96 \%) ; \mathrm{mp}=92{ }^{\circ} \mathrm{C} ;{ }^{1} \mathrm{H}$ NMR $\left(\mathrm{CDCl}_{3}, 500.4 \mathrm{MHz}\right): \delta 7.99$ (br s, $\left.1 \mathrm{H}\right), 7.83$ (s, 1 H), 7.65 (dd, $J=7.6,1.4 \mathrm{~Hz}, 2 \mathrm{H}), 7.60$ (d, $J=7.7 \mathrm{~Hz}, 1 \mathrm{H}), 7.46-7.40$ (m, $3 \mathrm{H}), 7.37$ (ddd, $J$ $=8.3,6.8,1.4 \mathrm{~Hz}, 1 \mathrm{H}), 7.26(\mathrm{~d}, J=7.8 \mathrm{~Hz}, 1 \mathrm{H}), 7.17(\mathrm{ddd}, J=7.9,7.3,1.1 \mathrm{~Hz}, 1 \mathrm{H}), 5.26$ (br s, $1 \mathrm{H}), 3.13(\mathrm{td}, J=6.4,6.3 \mathrm{~Hz}, 2 \mathrm{H}), 2.73(\mathrm{t}, J=7.6 \mathrm{~Hz}, 2 \mathrm{H}), 1.80(\mathrm{~s}, 3 \mathrm{H}), 1.63(\mathrm{tt}, J=$ 7.4, $7.2 \mathrm{~Hz}, 2 \mathrm{H}) \mathrm{ppm} ;{ }^{13} \mathrm{C}\left\{{ }^{1} \mathrm{H}\right\} \mathrm{NMR}\left(\mathrm{CDCl}_{3}, 125.8 \mathrm{MHz}\right): \delta 169.9,142.5,139.1,132.3$, $128.8,128.4,128.0,127.6,125.3,121.1,117.7,39.0,32.1,31.1,23.2 \mathrm{ppm} ;{ }^{11} \mathrm{~B}$ NMR (acetone, $128.4 \mathrm{MHz}$ ): $\delta 35.8$ ppm; IR: $v=3281,1650,1614,1597,1565,1453,1429,1365$, $1347,1286,1239,1208,948,752,731,701 \mathrm{~cm}^{-1}$; HRMS (ESI) m/z calc. for $\mathrm{C}_{19} \mathrm{H}_{22} \mathrm{BN}_{2} \mathrm{O}[\mathrm{M}$ $+\mathrm{H}]^{+} 305.1825$, found 305.1830 .<smiles>CC(=O)OCCCC1=Cc2ccccc2NB1c1ccccc1</smiles>

3-(3-Acetoxypropyl)-2-phenyl-2,1-borazanaphthalene (5c): obtained as a thick white wax (139 mg, 91\%); ${ }^{1} \mathrm{H}$ NMR $\left(\mathrm{CDCl}_{3}, 500.4 \mathrm{MHz}\right): \delta 7.93$ (br s, $\left.1 \mathrm{H}\right), 7.85(\mathrm{~s}, 1 \mathrm{H}), 7.67$ (dd, $J=$ 7.8, $1.6 \mathrm{~Hz}, 2 \mathrm{H}), 7.62(\mathrm{~d}, J=7.7 \mathrm{~Hz}, 1 \mathrm{H}), 7.46-7.41(\mathrm{~m}, 3 \mathrm{H}), 7.39$ (ddd, $J=8.2,7.2,1.4$ $\mathrm{Hz}, 1 \mathrm{H}), 7.26(\mathrm{~d}, J=8.2 \mathrm{~Hz}, 1 \mathrm{H}), 7.19(\mathrm{ddd}, J=7.8,7.1,1.0 \mathrm{~Hz}, 1 \mathrm{H}), 4.02(\mathrm{t}, J=6.6 \mathrm{~Hz}, 2$ $\mathrm{H}), 2.80(\mathrm{t}, J=7.9 \mathrm{~Hz}, 2 \mathrm{H}), 1.98(\mathrm{~s}, 3 \mathrm{H}), 1.81(\mathrm{tt}, J=7.8,6.4 \mathrm{~Hz}, 2 \mathrm{H}) \mathrm{ppm} ;{ }^{13} \mathrm{C}\left\{{ }^{1} \mathrm{H}\right\} \mathrm{NMR}$ $\left(\mathrm{CDCl}_{3}, 125.8 \mathrm{MHz}\right): \delta 171.1,142.3,139.1,132.3,128.8,128.4,128.0,127.6,125.3,121.2$, 117.7, 64.1, 31.4, 29.9, 20.9 ppm; ${ }^{11} \mathrm{~B}$ NMR (acetone, $128.4 \mathrm{MHz}$ ): $\delta 36.2 \mathrm{ppm}$; IR: $v=1721$, 1614, 1597, 1567, 1452, 1427, 1385, 1364, 1347, 1238, 1035, 999, 946, 918, 854, $751 \mathrm{~cm}^{-1}$; HRMS (ESI) $\mathrm{m} / \mathrm{z}$ calc. for $\mathrm{C}_{19} \mathrm{H}_{20} \mathrm{BNNaO}_{2}[\mathrm{M}+\mathrm{Na}]^{+} 328.1485$, found 328.1496 . 


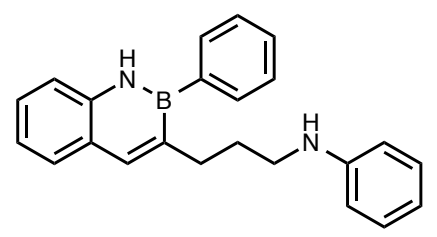

3-(3-(Phenylamino)propyl)-2-phenyl-2,1-borazanaphthalene (5d): obtained as a brown oil $(147 \mathrm{mg}, 87 \%) ;{ }^{1} \mathrm{H}$ NMR $\left(\mathrm{CDCl}_{3}, 500.4 \mathrm{MHz}\right): \delta 8.00$ (br s, $\left.1 \mathrm{H}\right), 7.96$ (s, $\left.1 \mathrm{H}\right), 7.79-7.75$ (m, $2 \mathrm{H}), 7.72(\mathrm{~d}, J=8.0 \mathrm{~Hz}, 1 \mathrm{H}), 7.58-7.54(\mathrm{~m}, 3 \mathrm{H}), 7.48(\mathrm{t}, J=7.4 \mathrm{~Hz}, 1 \mathrm{H}), 7.33-7.21$ (m, 4 H), $6.78(\mathrm{t}, J=7.2 \mathrm{~Hz}, 1 \mathrm{H}), 6.59(\mathrm{~d}, J=8.1 \mathrm{~Hz}, 2 \mathrm{H}), 3.51(\mathrm{br} \mathrm{s}, 1 \mathrm{H}), 3.15(\mathrm{t}, J=7.0 \mathrm{~Hz}, 2$ $\mathrm{H}), 2.94(\mathrm{t}, J=7.5 \mathrm{~Hz}, 2 \mathrm{H}), 1.87(\mathrm{tt}, J=7.5,7.0 \mathrm{~Hz}, 2 \mathrm{H}) \mathrm{ppm} ;{ }^{13} \mathrm{C}\left\{{ }^{1} \mathrm{H}\right\} \mathrm{NMR}\left(\mathrm{CDCl}_{3}\right.$, $125.8 \mathrm{MHz}): \delta 148.4,142.3,139.2,132.4,129.2,128.9,128.6,128.1,127.6,125.4,121.3$, $117.8,117.0,112.7,43.5,32.7,31.0 \mathrm{ppm} ;{ }^{11} \mathrm{~B}$ NMR (acetone, $128.4 \mathrm{MHz}$ ): $\delta 35.9 \mathrm{ppm}$; IR: $v$ $=1613,1600,1565,1504,1473,1451,1423,1345,1320,1282,1255,1240,1206,1179,944$, $765 \mathrm{~cm}^{-1}$; HRMS (ESI) m/z calc. for $\mathrm{C}_{23} \mathrm{H}_{24} \mathrm{BN}_{2}[\mathrm{M}+\mathrm{H}]^{+} 339.2031$, found 339.2033.

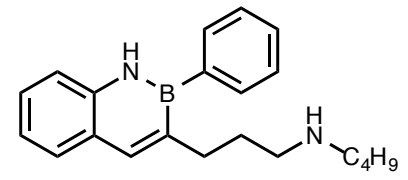

3-(3-(Butylamino)propyl)-2-phenyl-2,1-borazanaphthalene (5e): obtained as a light brown powder (141 mg, 89\%); ${ }^{1} \mathrm{H}$ NMR $\left(\mathrm{CDCl}_{3}, 500.4 \mathrm{MHz}\right): \delta 7.98$ (br s, $\left.1 \mathrm{H}\right), 7.88(\mathrm{~s}, 1 \mathrm{H}), 7.70$ $(\mathrm{dd}, J=7.8,1.5 \mathrm{~Hz}, 2 \mathrm{H}), 7.65(\mathrm{~d}, J=8.2 \mathrm{~Hz}, 1 \mathrm{H}), 7.49-7.43(\mathrm{~m}, 3 \mathrm{H}), 7.40$ (ddd, 8.1, 7.4, $1.7 \mathrm{~Hz}, 1 \mathrm{H}), 7.27(\mathrm{~d}, J=8.3 \mathrm{~Hz}, 1 \mathrm{H}), 7.21(\mathrm{ddd}, J=7.8,7.2,1.0 \mathrm{~Hz}, 1 \mathrm{H}), 2.79$ (t, $J=8.1$ $\mathrm{Hz}, 2 \mathrm{H}), 2.62(\mathrm{t}, J=7.3 \mathrm{~Hz}, 2 \mathrm{H}), 2.54$ (t, $J=7.5 \mathrm{~Hz}, 2 \mathrm{H}), 1.98$ (br s, $1 \mathrm{H}), 1.73$ (tt, $J=7.5$, $7.3 \mathrm{~Hz}, 2 \mathrm{H}), 1.48-1.40(\mathrm{~m}, 2 \mathrm{H}), 1.37-1.27(\mathrm{~m}, 2 \mathrm{H}), 0.92(\mathrm{t}, J=7.5 \mathrm{~Hz}, 3 \mathrm{H}) \mathrm{ppm} ;{ }^{13} \mathrm{C}\left\{{ }^{1} \mathrm{H}\right\}$ NMR $\left(\mathrm{CDCl}_{3}, 125.8 \mathrm{MHz}\right): \delta 142.0,139.1,132.3,128.7,128.4,127.9,127.4,125.4,121.1$, 117.6, 49.6, 49.4, 32.8, 32.0, 31.3, 20.4, $14.0 \mathrm{ppm} ;{ }^{11} \mathrm{~B}$ NMR (acetone, $128.4 \mathrm{MHz}$ ): $\delta 35.0$ ppm; IR: $v=2954,2927,2857,1615,1597,1567,1454,1423,1346,1283,1240,1207,954$, 943, 910, $764 \mathrm{~cm}^{-1}$; HRMS (ESI) $\mathrm{m} / \mathrm{z}$ calc. for $\mathrm{C}_{21} \mathrm{H}_{28} \mathrm{BN}_{2}[\mathrm{M}+\mathrm{H}]^{+} 319.2346$, found 319.2333 .<smiles>C1=Cc2ccccc2NB1C1=CC2CCC(C1)C2</smiles>

3-(exo-Bicyclo[2.2.1]heptan-2-yl)-2-phenyl-2,1-borazanaphthalene (5f): obtained as an off white solid (144 mg, 96\%); mp $=62{ }^{\circ} \mathrm{C} ;{ }^{1} \mathrm{H} \mathrm{NMR}\left(\mathrm{CDCl}_{3}, 500.4 \mathrm{MHz}\right): \delta 7.84$ (br s, $\left.1 \mathrm{H}\right)$, $7.81(\mathrm{~s}, 1 \mathrm{H}), 7.68-7.61(\mathrm{~m}, 3 \mathrm{H}), 7.46-7.38(\mathrm{~m}, 3 \mathrm{H}), 7.34(\mathrm{ddd}, J=8.2,6.9,1.5 \mathrm{~Hz}, 1 \mathrm{H})$, $7.22-7.19(\mathrm{~m}, 1 \mathrm{H}), 7.17(\mathrm{ddd}, J=8.1,6.9,1.1 \mathrm{~Hz}, 1 \mathrm{H}), 2.98(\mathrm{dd}, J=8.9,6.0 \mathrm{~Hz}, 1 \mathrm{H})$, 2.35-2.33 (m, $1 \mathrm{H}), 2.32-2.30(\mathrm{~m}, 1 \mathrm{H}), 1.66-1.44(\mathrm{~m}, 5 \mathrm{H}), 1.30-1.23(\mathrm{~m}, 1 \mathrm{H}), 1.21-1.11$ $(\mathrm{m}, 2 \mathrm{H}) \mathrm{ppm} ;{ }^{13} \mathrm{C}\left\{{ }^{1} \mathrm{H}\right\}$ NMR $\left(\mathrm{CDCl}_{3}, 125.8 \mathrm{MHz}\right): \delta 138.6,137.4,132.4,1291,128.1$, $127.8,127.2,125.4,121.1,117.5,44.9,42.4,38.7,37.2,35.5,30.2,29.0$ ppm; ${ }^{11} \mathrm{~B}$ NMR (acetone, 128.4 MHz): $\delta 36.6$ ppm; IR: $v=2946,2866,1612,1597,1563,1453,1419,1345$, $1307,1235,1206,940,909,763,747,700 \mathrm{~cm}^{-1}$; HRMS (ESI) $\mathrm{m} / \mathrm{z}$ calc. for $\mathrm{C}_{21} \mathrm{H}_{23} \mathrm{BN}[\mathrm{M}+$ $\mathrm{H}]^{+} 300.1924$, found 300.1928 . 


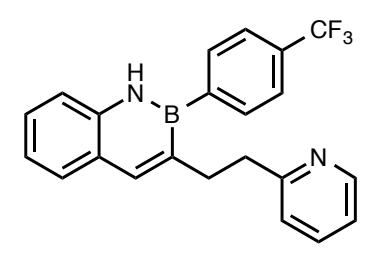

3-(2-Pyridin-2-yl)ethyl)-2-(4-trifluoromethylphenyl)-2,1-borazanaphthalene (6): obtained as a white solid $(145 \mathrm{mg}, 77 \%) ; \mathrm{mp}=100{ }^{\circ} \mathrm{C} ;{ }^{1} \mathrm{H}$ NMR (acetone- $d_{6}, 500.4 \mathrm{MHz}$ ): $\delta 9.71(\mathrm{br} \mathrm{s}$, $1 \mathrm{H}), 8.44(\mathrm{~d}, J=4.2 \mathrm{~Hz}, 1 \mathrm{H}), 8.01-7.87(\mathrm{~m}, 3 \mathrm{H}), 7.71(\mathrm{~d}, J=8.6 \mathrm{~Hz}, 2 \mathrm{H}), 7.67(\mathrm{~d}, J=7.7$ $\mathrm{Hz}, 1 \mathrm{H}$ ), 7.61 (d, $J=7.8 \mathrm{~Hz}, 1 \mathrm{H}), 7.57$ (ddd, $J=7.8,7.7,1.8 \mathrm{~Hz}, 1 \mathrm{H}), 7.40$ (ddd, $J=7.9$, 7.5, 1.7 Hz, $1 \mathrm{H}), 7.18$ (t, $J=7.9 \mathrm{~Hz}, 1 \mathrm{H}), 7.09-7.00$ (m, $2 \mathrm{H}), 3.13(\mathrm{~d}, J=8.6 \mathrm{~Hz}, 2 \mathrm{H}), 2.93$ $(\mathrm{dd}, J=9.6,7.3 \mathrm{~Hz}, 2 \mathrm{H}) \mathrm{ppm} ;{ }^{13} \mathrm{C}\left\{{ }^{1} \mathrm{H}\right\}$ NMR (acetone- $d_{6}, 125.8 \mathrm{MHz}$ ): $\delta 161.5,149.0$, $142.5,139.8,135.9,133.2,129.4$ (q, $J=32.4 \mathrm{~Hz}$ ), 128.7, 127.6, 125.5, 124.7 (q, $J=271.8$ $\mathrm{Hz}), 124.0$ (q, $J=4.0 \mathrm{~Hz}$ ), 122.5, 121.1, 120.9, 118.2, 39.5, $34.9 \mathrm{ppm} ;{ }^{11} \mathrm{~B}$ NMR (acetone, $128.4 \mathrm{MHz}$ ): $\delta 35.2 \mathrm{ppm} ;{ }^{19} \mathrm{~F}\left\{{ }^{1} \mathrm{H}\right\}$ NMR (acetone- $\left.d_{6}, 470.8 \mathrm{MHz}\right):-62.9 \mathrm{ppm} ; \mathrm{IR}: v=1595$, 1571, 1459, 1438, 1322, 1293, 1150, 1112, 1104, 1063, 1019, 969, 953, 835, 763, $749 \mathrm{~cm}^{-1}$; HRMS (ESI) $\mathrm{m} / \mathrm{z}$ calc. for $\mathrm{C}_{22} \mathrm{H}_{19} \mathrm{BF}_{3} \mathrm{~N}_{2}[\mathrm{M}+\mathrm{H}]^{+} 379.1593$, found 379.1599 .

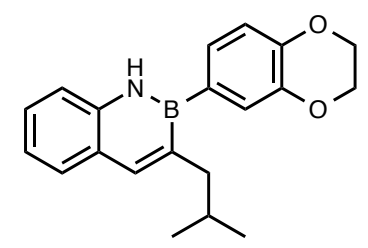

3-(Isobutyl)-2-(2,3-dihydro-1,4-benzodioxin-6-yl)-2,1-borazanaphthalene (7): obtained as a white solid (78 mg, 94\%), starting from 1f $(69 \mathrm{mg}, 0.26 \mathrm{mmol}) ; \mathrm{mp}=78{ }^{\circ} \mathrm{C} ;{ }^{1} \mathrm{H} \mathrm{NMR}$ $\left(\mathrm{CDCl}_{3}, 500.4 \mathrm{MHz}\right): \delta 8.09($ br s, $1 \mathrm{H}), 7.77(\mathrm{~s}, 1 \mathrm{H}), 7.60(\mathrm{~d}, J=7.5 \mathrm{~Hz}, 1 \mathrm{H}), 7.33$ (ddd, $J=$ 8.2, 7.0, 1.1 Hz, $1 \mathrm{H}), 7.20(\mathrm{~d}, J=8.3 \mathrm{~Hz}, 1 \mathrm{H}), 7.15(\mathrm{t}, J=7.2 \mathrm{~Hz}, 1 \mathrm{H}), 8.97(\mathrm{dd}, J=5.8,3.2$ $\mathrm{Hz}, 1 \mathrm{H}), 6.92-6.86(\mathrm{~m}, 2 \mathrm{H}), 4.26-4.17(\mathrm{~m}, 4 \mathrm{H}), 2.52$ (d, $J=7.2 \mathrm{~Hz}, 2 \mathrm{H}), 1.67$ (tsept, 6.6, $7.2 \mathrm{~Hz}, 1 \mathrm{H}), 0.79(\mathrm{~d}, J=6.6 \mathrm{~Hz}, 6 \mathrm{H}) \mathrm{ppm} ;{ }^{13} \mathrm{C}\left\{{ }^{1} \mathrm{H}\right\} \mathrm{NMR}\left(\mathrm{CDCl}_{3}, 125.8 \mathrm{MHz}\right): \delta 145.3$, 143.1, 142.3, 138.8, 128.7, 127.1, 125.8, 125.4, 121.3, 121.0, 117.6, 117.5, 64.3, 64.2, 45.1, 29.2, $22.6 \mathrm{ppm} ;{ }^{11} \mathrm{~B}$ NMR (acetone, $128.4 \mathrm{MHz}$ ): $\delta 36.5 \mathrm{ppm}$; IR: $v=2946,1614,1597$, $1565,1454,1437,1422,1377,1280,1252,1217,1200,1093,1082,930,895,843 \mathrm{~cm}^{-1}$; HRMS (ESI) $\mathrm{m} / \mathrm{z}$ calc. for $\mathrm{C}_{20} \mathrm{H}_{23} \mathrm{BNO}_{2}[\mathrm{M}+\mathrm{H}]^{+} 320.1822$, found 320.1836 .<smiles>COCCCC1=Cc2ccccc2NB1c1c(F)cccc1F</smiles>

3-(3-Methoxypropyl)-2-(2,6-difluorophenyl)-2,1-borazanaphthalene (8): obtained as a white solid from $(150 \mathrm{mg}, 96 \%) ; \mathrm{mp}=73{ }^{\circ} \mathrm{C} ;{ }^{1} \mathrm{H}$ NMR (acetone- $d_{6}, 500.4 \mathrm{MHz}$ ): $\delta 9.91$ (br s, $1 \mathrm{H}), 7.96(\mathrm{~s}, 1 \mathrm{H}), 7.71(\mathrm{~d}, J=9.1 \mathrm{~Hz}, 1 \mathrm{H}), 7.61(\mathrm{~d}, J=8.0 \mathrm{~Hz}, 1 \mathrm{H}), 7.47-7.40(\mathrm{~m}, 2 \mathrm{H})$, 7.22 (ddd, $J=8.1,7.0,1.1 \mathrm{~Hz}, 1 \mathrm{H}), 7.03-6.95(\mathrm{~m}, 2 \mathrm{H}), 3.25(\mathrm{t}, J=6.7 \mathrm{~Hz}, 2 \mathrm{H}), 3.15(\mathrm{~s}, 3$ $\mathrm{H}), 2.63(\mathrm{t}, J=8.2 \mathrm{~Hz}, 2 \mathrm{H}), 1.69(\mathrm{tt}, J=8.3,6.7 \mathrm{~Hz}, 2 \mathrm{H}) \mathrm{ppm} ;{ }^{13} \mathrm{C}\left\{{ }^{1} \mathrm{H}\right\} \mathrm{NMR}\left(\mathrm{CDCl}_{3}\right.$, $125.8 \mathrm{MHz}): \delta 164.4(\mathrm{dd}, J=238.9,16.7 \mathrm{~Hz}), 141.7,139.4,131.0(\mathrm{t}, J=10.1 \mathrm{~Hz}), 128.7$, $127.5,125.6,121.3,118.1,110.7(\mathrm{dd}, J=21.8,5.7 \mathrm{~Hz}), 71.8,57.3,31.4,30.6 \mathrm{ppm} ;{ }^{11} \mathrm{~B}$ NMR (acetone, $128.4 \mathrm{MHz}): \delta 33.6 \mathrm{ppm} ;{ }^{19} \mathrm{~F}\left\{{ }^{1} \mathrm{H}\right\}$ NMR (acetone- $d_{6}, 470.8 \mathrm{MHz}$ ): $-103.2 \mathrm{ppm}$; IR: $v=3274,1617,1573,1462,1444,1227,1213,1110,1101,1060,976,966,943,852,777$, $760 \mathrm{~cm}^{-1}$; HRMS (ESI) m/z calc. for $\mathrm{C}_{18} \mathrm{H}_{19} \mathrm{BF}_{2} \mathrm{NO}[\mathrm{M}+\mathrm{H}]^{+} 314.1528$, found 314.1536. 
<smiles>CC(=O)OCC1=Cc2ccccc2NB1c1ccc(Cl)cc1</smiles>

3-(Acetoxymethyl)-2-(4-chlorophenyl)-2,1-borazanaphthalene (9): obtained as a white solid (94 mg, 60\%); mp = $122{ }^{\circ} \mathrm{C} ;{ }^{1} \mathrm{H}$ NMR (acetone- $\left.d_{6}, 500.4 \mathrm{MHz}\right): \delta 9.73(\mathrm{br} \mathrm{s}, 1 \mathrm{H}), 8.13$ $(\mathrm{s}, 1 \mathrm{H}), 7.76-7.71(\mathrm{~m}, 3 \mathrm{H}), 7.65(\mathrm{~d}, J=8.7 \mathrm{~Hz}, 1 \mathrm{H}), 7.50-7.42(\mathrm{~m}, 3 \mathrm{H}), 7.22$ (ddd, $J=8.2$, 6.7, $1.1 \mathrm{~Hz}, 1 \mathrm{H}), 5.19(\mathrm{~s}, 2 \mathrm{H}), 2.03(\mathrm{~s}, 3 \mathrm{H}) \mathrm{ppm} ;{ }^{13} \mathrm{C}\left\{{ }^{1} \mathrm{H}\right\}$ NMR (acetone- $\left.d_{6}, 125.8 \mathrm{MHz}\right): \delta$ $169.9,142.9,140.5,134.6,134.2,129.2$, 128.6, 127.7, 124.6, 121.2, 118.3, 66.2, 20.0 ppm; ${ }^{11} \mathrm{~B}$ NMR (acetone, $128.4 \mathrm{MHz}$ ): $\delta 34.9$ ppm; IR: $v=3324,1720,1616,1587,1575,1454$, 1431, 1367, 1340, 1246, 1210, 1100, 1088, 1034, 1013, 961, $792 \mathrm{~cm}^{-1}$; HRMS (ESI) m/z calc. for $\mathrm{C}_{17} \mathrm{H}_{15} \mathrm{BClNNaO} 2[\mathrm{M}+\mathrm{Na}]^{+}$334.0782, found 334.0796.<smiles>FC(F)(F)c1ccc(B2C=Cc3ccccc3N2)cc1</smiles>

2-(4-(Trifluoromethyl)phenyl)-2,1-borazanaphthalene (10a): obtained as an off white powder $(0.42 \mathrm{~g}, 27 \%, 5.00 \mathrm{mmol}$ scale $) ; \mathrm{mp}=154{ }^{\circ} \mathrm{C} ;{ }^{1} \mathrm{H}$ NMR (DMSO- $\left.d_{6}, 500.4 \mathrm{MHz}\right): \delta$ $10.67($ br s, $1 \mathrm{H}), 8.28(\mathrm{~d}, J=6.6 \mathrm{~Hz}, 2 \mathrm{H}), 8.22(\mathrm{~d}, J=12.1 \mathrm{~Hz}, 1 \mathrm{H}), 7.82(\mathrm{~d}, J=7.7 \mathrm{~Hz}, 2$ H), 7.75 (t, $J=8.8 \mathrm{~Hz}, 2 \mathrm{H}), 7.51$ (ddd, $J=8.7,7.3,1.7 \mathrm{~Hz}, 1 \mathrm{H}), 7.28$ (dd, $J=11.9,1.5 \mathrm{~Hz}, 1$ H), 7.22 (ddd, $J=7.8,7.4,1.5 \mathrm{~Hz}, 1 \mathrm{H}) \mathrm{ppm} ;{ }^{13} \mathrm{C}\left\{{ }^{1} \mathrm{H}\right\}$ NMR (DMSO- $d_{6}, 125.8 \mathrm{MHz}$ ): $\delta$ 146.2, 141.1, 134.4, 130.0 (q, $J=31.1 \mathrm{~Hz}), 129.5,128.9,125.7,124.9$ (q, $J=273.3 \mathrm{~Hz})$, $124.8(\mathrm{q}, J=5.3 \mathrm{~Hz}), 121.4,119.2 \mathrm{ppm} ;{ }^{11} \mathrm{~B}$ NMR (acetone, $128.4 \mathrm{MHz}$ ): $\delta 33.8 \mathrm{ppm} ;{ }^{19} \mathrm{~F}$ $\left\{{ }^{1} \mathrm{H}\right\}$ NMR (DMSO- $\left.d_{6}, 470.8 \mathrm{MHz}\right):-61.2 \mathrm{ppm}$; IR: $v=1614,1560,1434,1326,1205,1168$, 1159, 1117, 1104, 1069, 1015, 989, 978, 838, 811, $761 \mathrm{~cm}^{-1}$; HRMS (ESI) $\mathrm{m} / \mathrm{z}$ calc. for $\mathrm{C}_{15} \mathrm{H}_{10} \mathrm{BF}_{3} \mathrm{~N}[\mathrm{M}-\mathrm{H}]^{+}$272.0858, found 272.0853.<smiles>C1=Cc2ccccc2NB1c1ccc2c(c1)OCCO2</smiles>

2-(2,3-Dihydro-1,4-benzodioxin-6-yl)-2,1-borazanaphthalene (10b): obtained as an off white powder $\left(1.73 \mathrm{~g}, 84 \%, 7.80 \mathrm{mmol}\right.$ scale) from $10 \mathrm{~b} ; \mathrm{mp}=89{ }^{\circ} \mathrm{C} ;{ }^{1} \mathrm{H}$ NMR (DMSO- $d_{6}$, $500.4 \mathrm{MHz}$ ): $\delta 10.23$ (br s, $1 \mathrm{H}), 8.07$ (d, $J=10.6 \mathrm{~Hz}, 1 \mathrm{H}), 7.68(\mathrm{~d}, J=8.7 \mathrm{~Hz}, 1 \mathrm{H}), 7.64(\mathrm{~d}$, $J=7.8 \mathrm{~Hz}, 1 \mathrm{H}), 7.62-7.59(\mathrm{~m}, 1 \mathrm{H}), 7.54(\mathrm{dd}, J=8.1,1.4 \mathrm{~Hz}, 1 \mathrm{H}), 7.42(\mathrm{ddd}, J=8.4,6.9$, $1.4 \mathrm{~Hz}, 1 \mathrm{H}), 7.17(\mathrm{dd}, J=11.2,1.6 \mathrm{~Hz}, 1 \mathrm{H}), 7.13(\mathrm{ddd}, J=8.4,7.2,1.0 \mathrm{~Hz}, 1 \mathrm{H}), 6.93(\mathrm{~d}, J$ $=7.9 \mathrm{~Hz}, 1 \mathrm{H}), 4.28(\mathrm{~s}, 4 \mathrm{H}) \mathrm{ppm} ;{ }^{13} \mathrm{C}\left\{{ }^{1} \mathrm{H}\right\}$ NMR $\left(\mathrm{DMSO}-d_{6}, 125.8 \mathrm{MHz}\right): \delta 145.4,145.3$, $143.7,141.7,129.3,128.6,127.2,125.3,122.2,120.8,118.9,117.2,64.7,64.4 \mathrm{ppm} ;{ }^{11} \mathrm{~B}$ NMR (acetone, $128.4 \mathrm{MHz}$ ): $\delta 33.8 \mathrm{ppm}$; IR: $v=3341,1613,1605,1596,1564,1510,1455$, 1438, 1303, 1284, 1250, 1216, 1127, 1068, 1047, 898, $803 \mathrm{~cm}^{-1}$; HRMS (CI) m/z calc. for $\mathrm{C}_{16} \mathrm{H}_{14} \mathrm{BNO}_{2}[\mathrm{M}]^{+}$263.1118, found 263.1132.<smiles>Fc1cccc(F)c1B1C=Cc2ccccc2N1</smiles>

2-(2,6-Difluorophenyl)-2,1-borazanaphthalene (10c): obtained as an off white powder $(0.67 \mathrm{~g}, 56 \%, 5.00 \mathrm{mmol} \mathrm{scale}) ; \mathrm{mp}=54{ }^{\circ} \mathrm{C} ;{ }^{1} \mathrm{H}$ NMR (DMSO- $\left.d_{6}, 500.4 \mathrm{MHz}\right): \delta 10.69$ (br s, 
$1 \mathrm{H}), 8.20(\mathrm{~d}, J=10.9 \mathrm{~Hz}, 1 \mathrm{H}), 7.76(\mathrm{~d}, J=7.3 \mathrm{~Hz}, 1 \mathrm{H}), 7.68(\mathrm{~d}, J=8.5 \mathrm{~Hz}, 1 \mathrm{H}), 7.55-7.48$ $(\mathrm{m}, 2 \mathrm{H}), 7.24(\mathrm{t}, J=7.6 \mathrm{~Hz}, 1 \mathrm{H}), 7.12(\mathrm{t}, J=7.3 \mathrm{~Hz}, 2 \mathrm{H}), 7.02(\mathrm{~d}, J=11.5 \mathrm{~Hz}, 1 \mathrm{H}) \mathrm{ppm}$; ${ }^{13} \mathrm{C}\left\{{ }^{1} \mathrm{H}\right\}$ NMR (DMSO- $\left.d_{6}, 125.8 \mathrm{MHz}\right): \delta 165.0(\mathrm{dd}, J=240.1,16.1 \mathrm{~Hz}), 145.5,140.7,132.5$ $(\mathrm{t}, J=12.0 \mathrm{~Hz}), 129.5,128.9,125.4,121.6,119.2,111.5(\mathrm{dd}, J=22.1,5.9 \mathrm{~Hz}) \mathrm{ppm} ;{ }^{19} \mathrm{~F}$ NMR (DMSO- $d_{6}, 470.8 \mathrm{MHz}$ ): $\delta-102.7 \mathrm{ppm} ;{ }^{11} \mathrm{~B}$ NMR (acetone, $\left.128.4 \mathrm{MHz}\right): \delta 32.3 \mathrm{ppm}$; IR: $v=$ $1615,1558,1463,1452,1429,1385,1345,1276,1247,1217,1202,990,973,943,810,779$ $\mathrm{cm}^{-1}$; HRMS (CI) m/z calc. for $\mathrm{C}_{14} \mathrm{H}_{10} \mathrm{BF}_{2} \mathrm{~N}[\mathrm{M}]^{+} 241.0874$, found 241.0871.

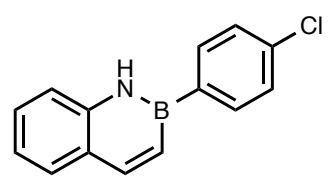

2-(4-Chlorophenyl)-2,1-borazanaphthalene (10d): obtained as an off white powder $(0.40 \mathrm{~g}$, $83 \%, 2.00 \mathrm{mmol} \mathrm{scale}$ ); $\mathrm{mp}=140{ }^{\circ} \mathrm{C} ;{ }^{1} \mathrm{H}$ NMR (DMSO- $\left.d_{6}, 500.4 \mathrm{MHz}\right): \delta 10.50$ (br s, $1 \mathrm{H}$ ), $8.17(\mathrm{~d}, J=11.5 \mathrm{~Hz}, 1 \mathrm{H}), 8.10(\mathrm{~d}, J=8.1 \mathrm{~Hz}, 2 \mathrm{H}), 7.73(\mathrm{~d}, J=8.3 \mathrm{~Hz}, 1 \mathrm{H}), 7.70(\mathrm{~d}, J=7.7$ $\mathrm{Hz}, 1 \mathrm{H}), 7.53$ (d, $J=8.3 \mathrm{~Hz}, 2 \mathrm{H}), 7.48$ (ddd, $J=8.5,7.2,1.5 \mathrm{~Hz}, 1 \mathrm{H}), 7.24$ (dd, $J=11.6$, $1.8 \mathrm{~Hz}, 1 \mathrm{H}$ ), 7.19 (ddd, $J=8.1,6.6,1.1 \mathrm{~Hz}, 1 \mathrm{H}$ ) ppm; ${ }^{13} \mathrm{C}\left\{{ }^{1} \mathrm{H}\right\}$ NMR (DMSO- $d_{6}, 125.8$ $\mathrm{MHz}): \delta 145.8,141.2,135.6,135.0,129.4,128.8,128.3,125.5,121.1,119.0$ ppm; ${ }^{11} \mathrm{~B}$ NMR (acetone, $128.4 \mathrm{MHz}$ ): $\delta 34.1 \mathrm{ppm}$; IR: $v=1611,1582,1556,1494,1434,1392,1345,1280$, $1223,1205,1117,1089,1010,975,822,808 \mathrm{~cm}^{-1}$; HRMS (ESI) m/z calc. for $\mathrm{C}_{14} \mathrm{H}_{11} \mathrm{BCl}_{2} \mathrm{~N}$ $[\mathrm{M}+\mathrm{Cl}]^{-} 274.0362$, found 274.0363 .<smiles>NNC(=O)c1ccc2c(c1)OC(CCF)(CCCF)O2</smiles>

Diisopropylammonium bis(catecholato)3,3,3-trifluoropropylsilicate (11a): obtained as a white powder $\left(6.31 \mathrm{~g}, 92 \%, 15.00 \mathrm{mmol}\right.$ scale), crystallized with 0.2 equiv THF, mp $237{ }^{\circ} \mathrm{C}$; ${ }^{1} \mathrm{H}$ NMR (DMSO- $\left.d_{6}, 500.4 \mathrm{MHz}\right): \delta 8.02$ (br s, $\left.2 \mathrm{H}\right), 6.59-6.55(\mathrm{~m}, 4 \mathrm{H}), 6.49-6.45(\mathrm{~m}, 4 \mathrm{H})$, 3.34 (sept, $J=6.9 \mathrm{~Hz}, 2 \mathrm{H}), 2.04-1.96(\mathrm{~m}, 2 \mathrm{H}), 1.19$ (d, $J=6.9 \mathrm{~Hz}, 12 \mathrm{H}), 0.68-0.62$ (m, 2 H) ppm; ${ }^{13} \mathrm{C}\left\{{ }^{1} \mathrm{H}\right\}$ NMR (DMSO- $\left.d_{6}, 125.8 \mathrm{MHz}\right): \delta 150.6,128.8(\mathrm{q}, J=277.9 \mathrm{~Hz}), 117.9$, 110.2, 46.8, 28.9 (q, $J=28.9 \mathrm{~Hz}), 19.2,9.54 \mathrm{ppm} ;{ }^{19} \mathrm{~F}\left\{{ }^{1} \mathrm{H}\right\} \mathrm{NMR}\left(\mathrm{CDCl}_{3}, 470.8 \mathrm{MHz}\right):-$ 67.3 ppm; IR: $v=2987,1707,1599,1484,1392,1349,1321,1297,1238,1167,1149,1098$, 1014, 938, 891, $811 \mathrm{~cm}^{-1}$; HRMS (ESI) $\mathrm{m} / \mathrm{z}$ calc. for $\mathrm{C}_{15} \mathrm{H}_{12} \mathrm{~F}_{3} \mathrm{O}_{4} \mathrm{Si}\left[\mathrm{M}-i-\mathrm{Pr}_{2} \mathrm{NH}_{2}\right]^{-}$ 341.0457 , found 341.0462 .<smiles></smiles>

Diisopropylammonium bis(catecholato)acetoxymethylsilicate (11b): obtained as a white powder $\left(1.71 \mathrm{~g}, 76 \%, 5.15 \mathrm{mmol}\right.$ scale), mp $127{ }^{\circ} \mathrm{C} ;{ }^{1} \mathrm{H}$ NMR (acetone- $d_{6}, 500.4 \mathrm{MHz}$ ): $\delta$ 7.43 (br s, 2 H), 6.69-6.63 (m, 4 H), 6.54-6.50 (m, 4 H), 3.82 (sept, $J=6.7$ Hz, 2 H), 3.80 (s, $2 \mathrm{H}), 1.74(\mathrm{~s}, 3 \mathrm{H}), 1.43(\mathrm{~d}, J=6.7 \mathrm{~Hz}, 12 \mathrm{H}) \mathrm{ppm} ;{ }^{13} \mathrm{C}\left\{{ }^{1} \mathrm{H}\right\}$ NMR (acetone- $\left.d_{6}, 125.8 \mathrm{MHz}\right)$ : $\delta 170.5,150.0,118.0,110.3,56.2,48.3,19.9,18.6 \mathrm{ppm}$; IR: $v=1725,1483,1393,1365$, 1347, 1294, 1235, 1148, 1098, 1038, 1014, 887, 814, 771, 738, $707 \mathrm{~cm}^{-1}$; HRMS (ESI) m/z calc. for $\mathrm{C}_{15} \mathrm{H}_{13} \mathrm{O}_{6} \mathrm{Si}\left[\mathrm{M}-i-\mathrm{Pr}_{2} \mathrm{NH}_{2}\right]^{-}$317.0481, found 317.0482 . 


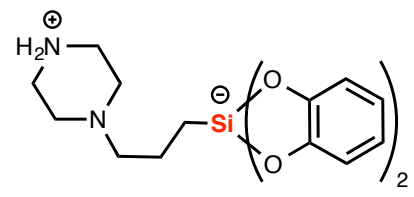

Bis(catecholato)3-(piperazin-1-ium-1-yl)propyl silicate (11c): obtained as a white powder (909 mg, 71\%, $3.44 \mathrm{mmol} \mathrm{scale),} \mathrm{mp}>260{ }^{\circ} \mathrm{C} ;{ }^{1} \mathrm{H}$ NMR (DMSO- $d_{6}, 500.4 \mathrm{MHz}$ ): $\delta 8.16$ (br $\mathrm{s}, 2 \mathrm{H}), 6.53-6.50(\mathrm{~m}, 4 \mathrm{H}), 6.46-6.41(\mathrm{~m}, 4 \mathrm{H}), 2.98-2.96(\mathrm{~m}, 4 \mathrm{H}), 2.43-2.42(\mathrm{~m}, 4 \mathrm{H}), 2.20$ $(\mathrm{t}, J=7.0 \mathrm{~Hz}, 2 \mathrm{H}), 1.36(\mathrm{tt}, J=7.7,7.7 \mathrm{~Hz}, 2 \mathrm{H}), 0.47(\mathrm{t}, J=7.0 \mathrm{~Hz}, 2 \mathrm{H}) \mathrm{ppm} ;{ }^{13} \mathrm{C}\left\{{ }^{1} \mathrm{H}\right\}$ NMR (DMSO- $\left.d_{6}, 125.8 \mathrm{MHz}\right): \delta 150.9,117.5,109.9,61.1,49.9,43.4,21.4,15.7$ ppm; IR: $v$ $=2812,1488,1457,1352,1243,1223,1198,1174,1104,1015,889,828,769,738,724,716$ $\mathrm{cm}^{-1}$; HRMS (ESI) m/z calc. for $\mathrm{C}_{19} \mathrm{H}_{24} \mathrm{~N}_{2} \mathrm{O}_{4} \mathrm{Si}[\mathrm{M}]^{+} 372.1505$, found 372.1506 .

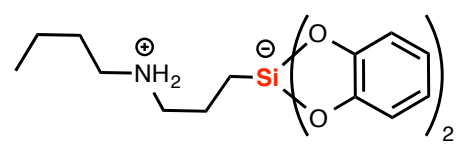

Bis(catecholato)3-(butylammonio)propyl silicate (11d): obtained as a white powder (6.86 g, 90\%, $21.25 \mathrm{mmol} \mathrm{scale}$ ), $\mathrm{mp} 225^{\circ} \mathrm{C} ;{ }^{1} \mathrm{H}$ NMR (DMSO- $d_{6}, 500.4 \mathrm{MHz}$ ): $\delta 8.00$ (br s, $2 \mathrm{H}$ ), 6.57-6.51 (m, 4 H), 6.48-6.42 (m, 4 H), 2.76-2.68 (m, 4 H), 1.58-1.49 (m, 2 H), 1.48-1.40 $(\mathrm{m}, 2 \mathrm{H}), 1.25(\mathrm{tt}, J=7.0,7.0 \mathrm{~Hz}, 2 \mathrm{H}), 0.84(\mathrm{t}, J=7.4 \mathrm{~Hz}, 3 \mathrm{H}), 0.52(\mathrm{t}, J=8.0 \mathrm{~Hz}, 2 \mathrm{H})$ ppm; ${ }^{13} \mathrm{C}\left\{{ }^{1} \mathrm{H}\right\}$ NMR (DMSO- $d_{6}, 125.8 \mathrm{MHz}$ ): $\delta 150.7,117.7,110.1,50.3,46.9,27.8,21.9$, 19.6, 15.3, 13.8 ppm; IR: $v=3031,1485,1460,1435,1352,1257,1236,1222,1194,1102$, $1015,888,830,753,730,697,656 \mathrm{~cm}^{-1}$; HRMS (ESI) m/z calc. for $\mathrm{C}_{19} \mathrm{H}_{24} \mathrm{NO}_{4} \mathrm{Si}[\mathrm{M}-\mathrm{H}]^{-}$ 358.1475 , found 358.1479 . 


\section{Spectral data}

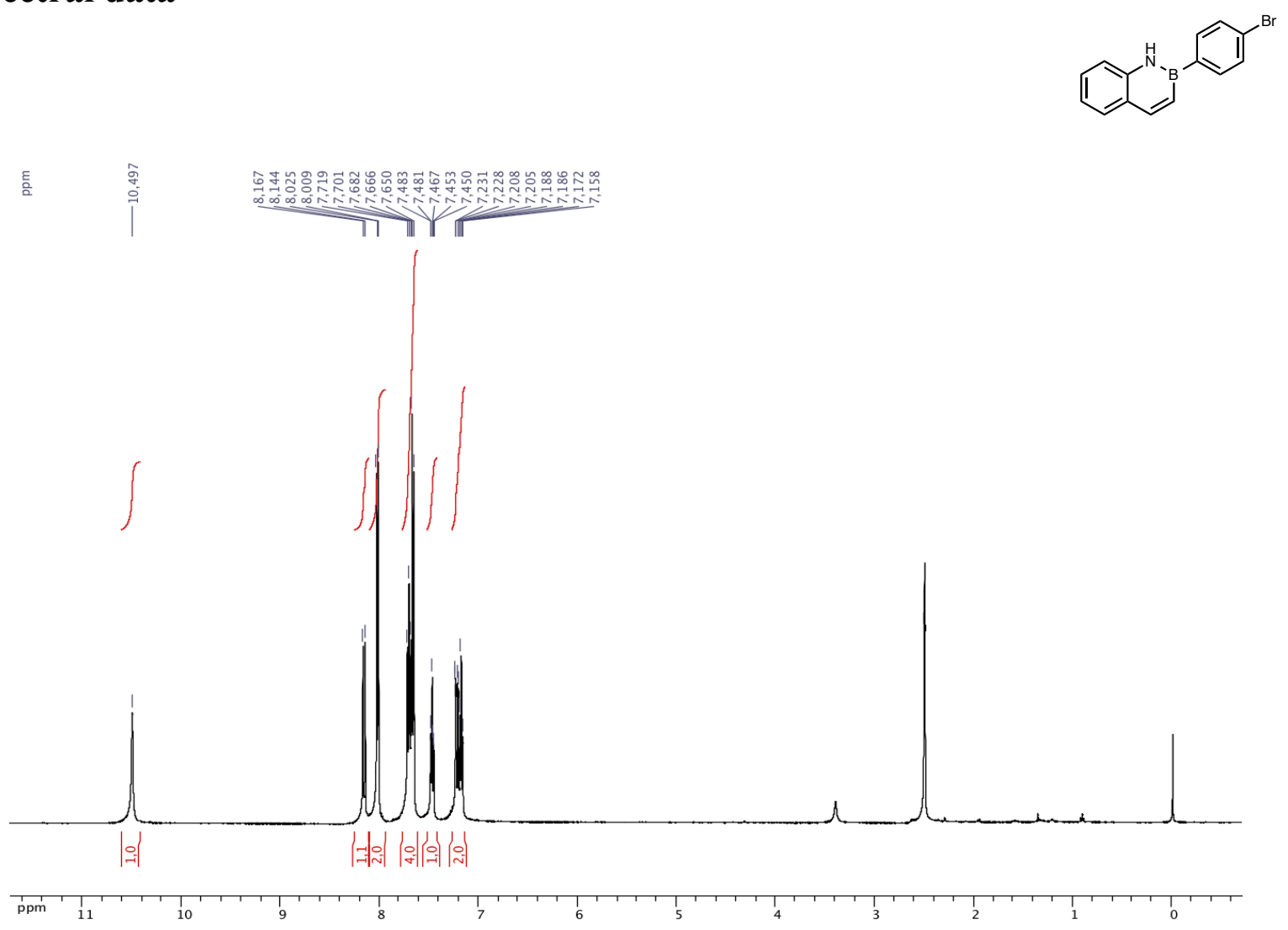

${ }^{1} \mathrm{H}$ NMR (DMSO- $d_{6}, 500.4 \mathrm{MHz}$ ) of 2-(4-bromophenyl)-2,1-borazanaphthalene (1a)

言

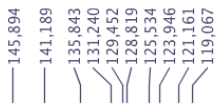
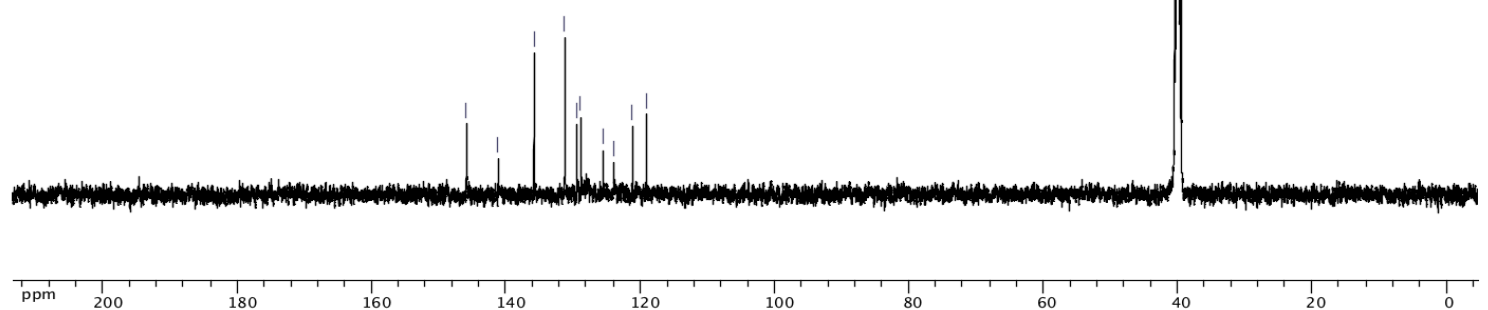

${ }^{13} \mathrm{C}\left\{{ }^{1} \mathrm{H}\right\}$ NMR (DMSO- $\left.d_{6}, 125.8 \mathrm{MHz}\right)$ of 2-(4-bromophenyl)-2,1-borazanaphthalene (1a) 

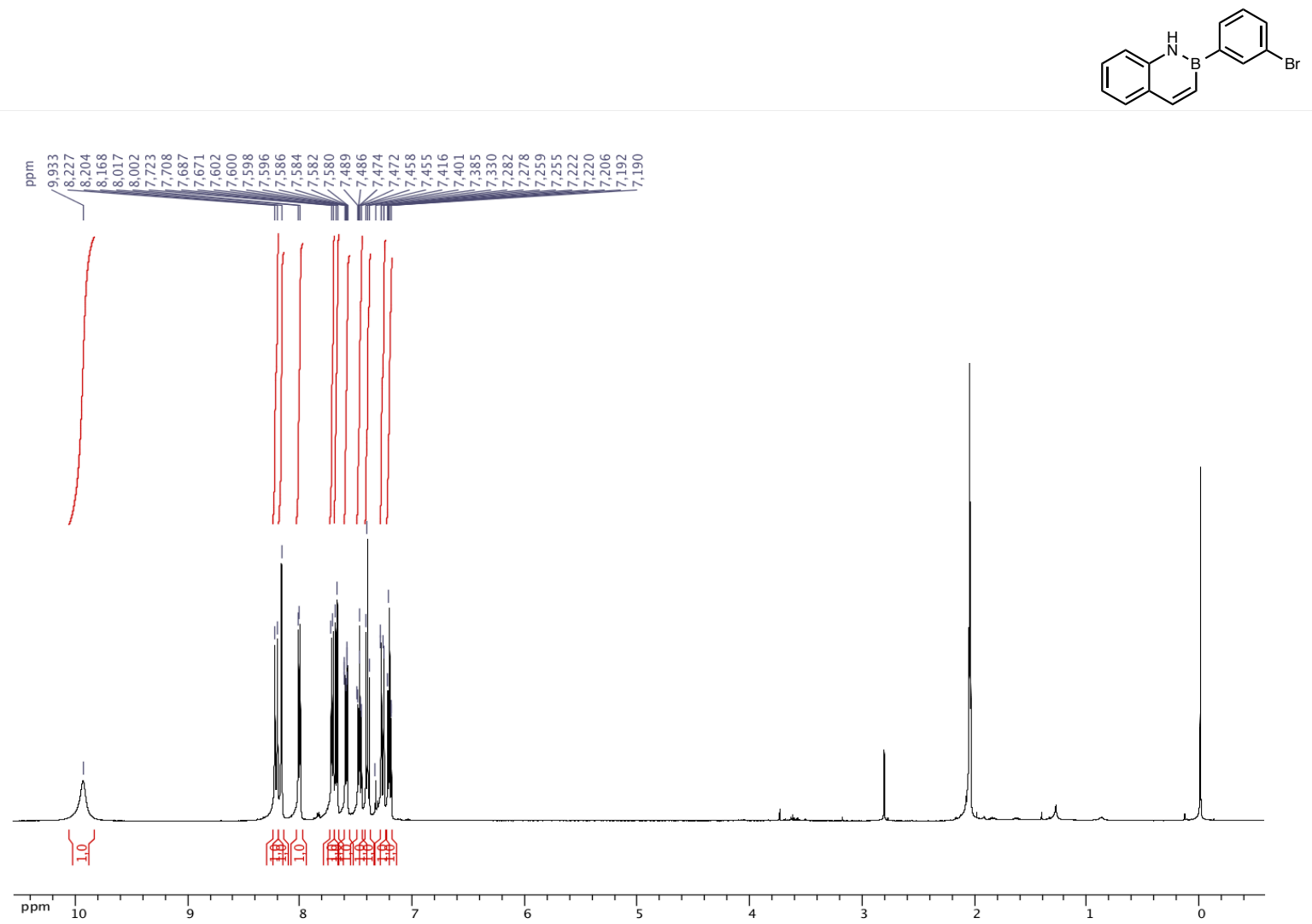

${ }^{1} \mathrm{H}$ NMR (acetone- $d_{6}, 500.4 \mathrm{MHz}$ ) of 2-(3-bromophenyl)-2,1-borazanaphthalene (1b)
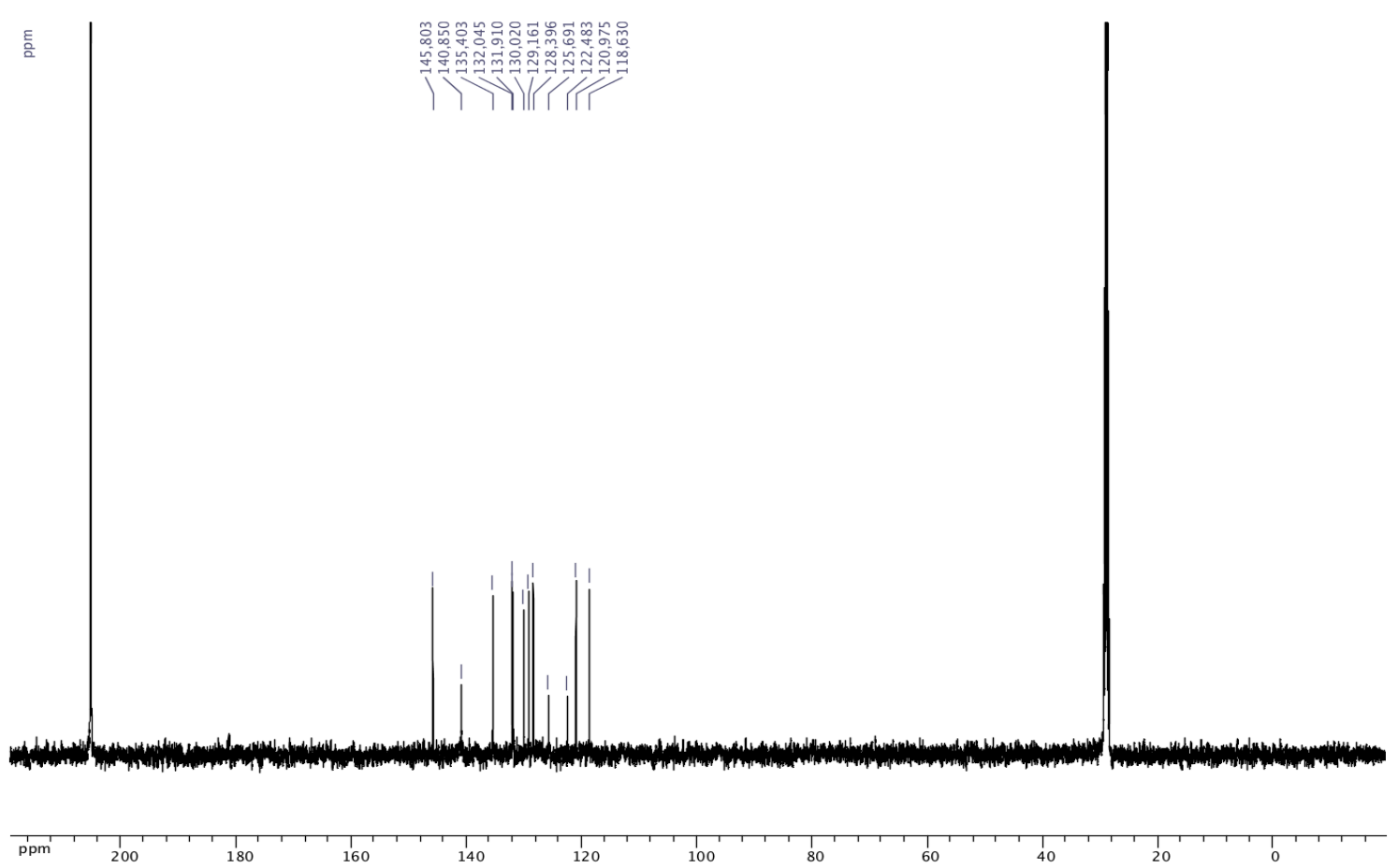

${ }^{13} \mathrm{C}\left\{{ }^{1} \mathrm{H}\right\}$ NMR (acetone- $d_{6}, 125.8 \mathrm{MHz}$ ) of 2-(3-bromophenyl)-2,1-borazanaphthalene (1b) 

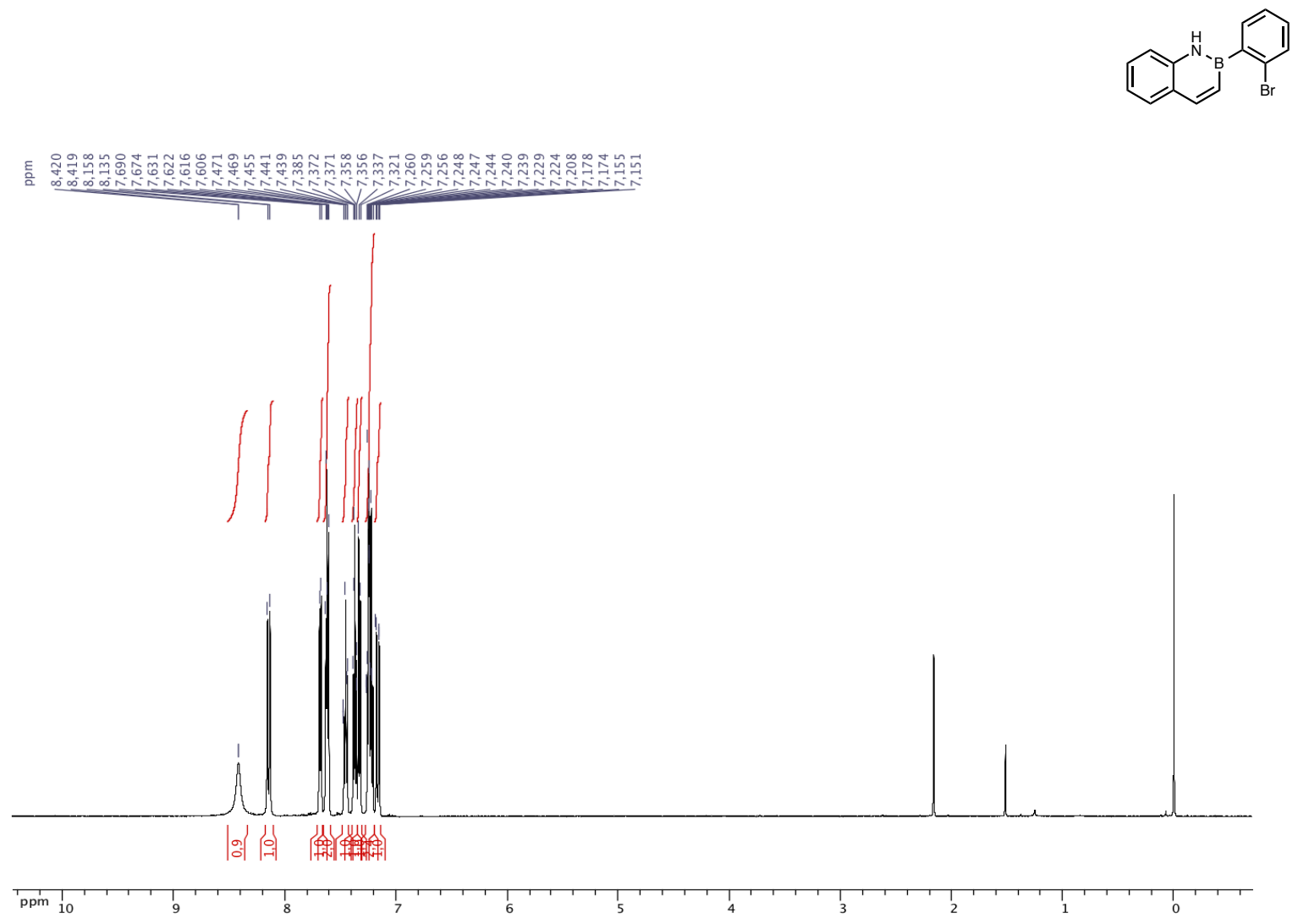

${ }^{1} \mathrm{H}$ NMR $\left(\mathrm{CDCl}_{3}, 500.4 \mathrm{MHz}\right)$ of 2-(2-bromophenyl)-2,1-borazanaphthalene (1c)

言

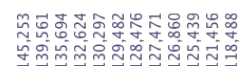

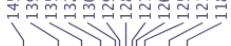
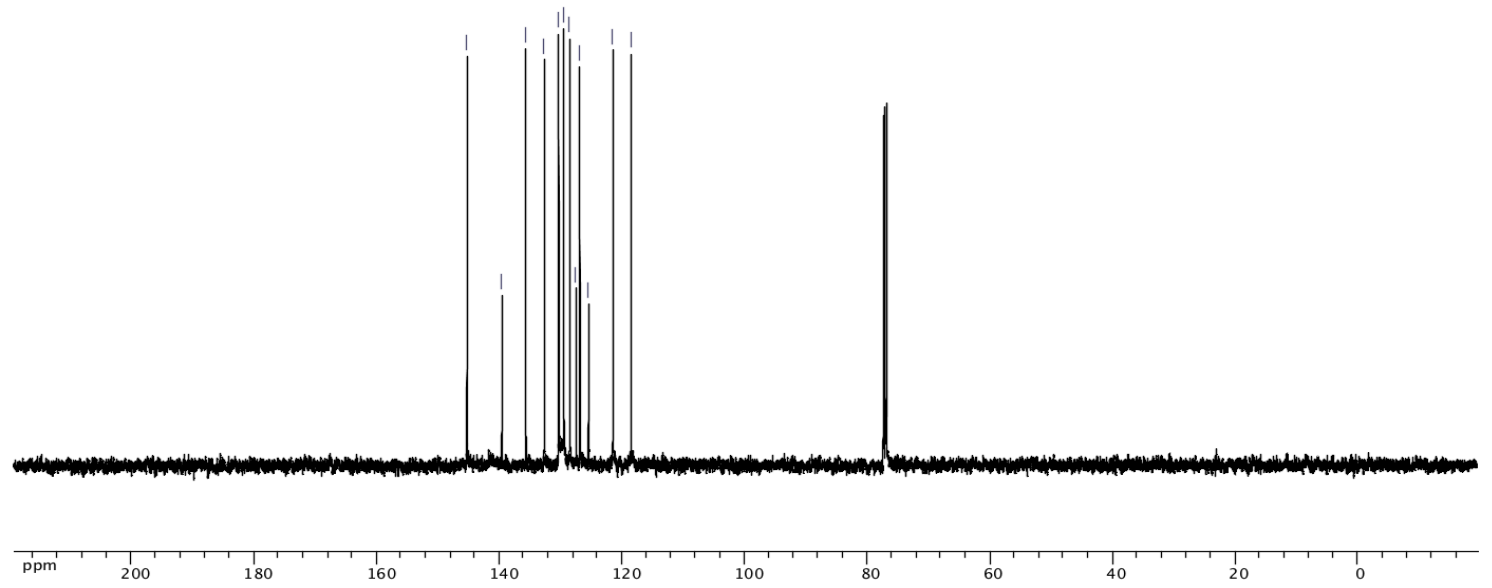

${ }^{13} \mathrm{C}\left\{{ }^{1} \mathrm{H}\right\}$ NMR $\left(\mathrm{CDCl}_{3}, 125.8 \mathrm{MHz}\right)$ of 2-(2-bromophenyl)-2,1-borazanaphthalene (1c) 


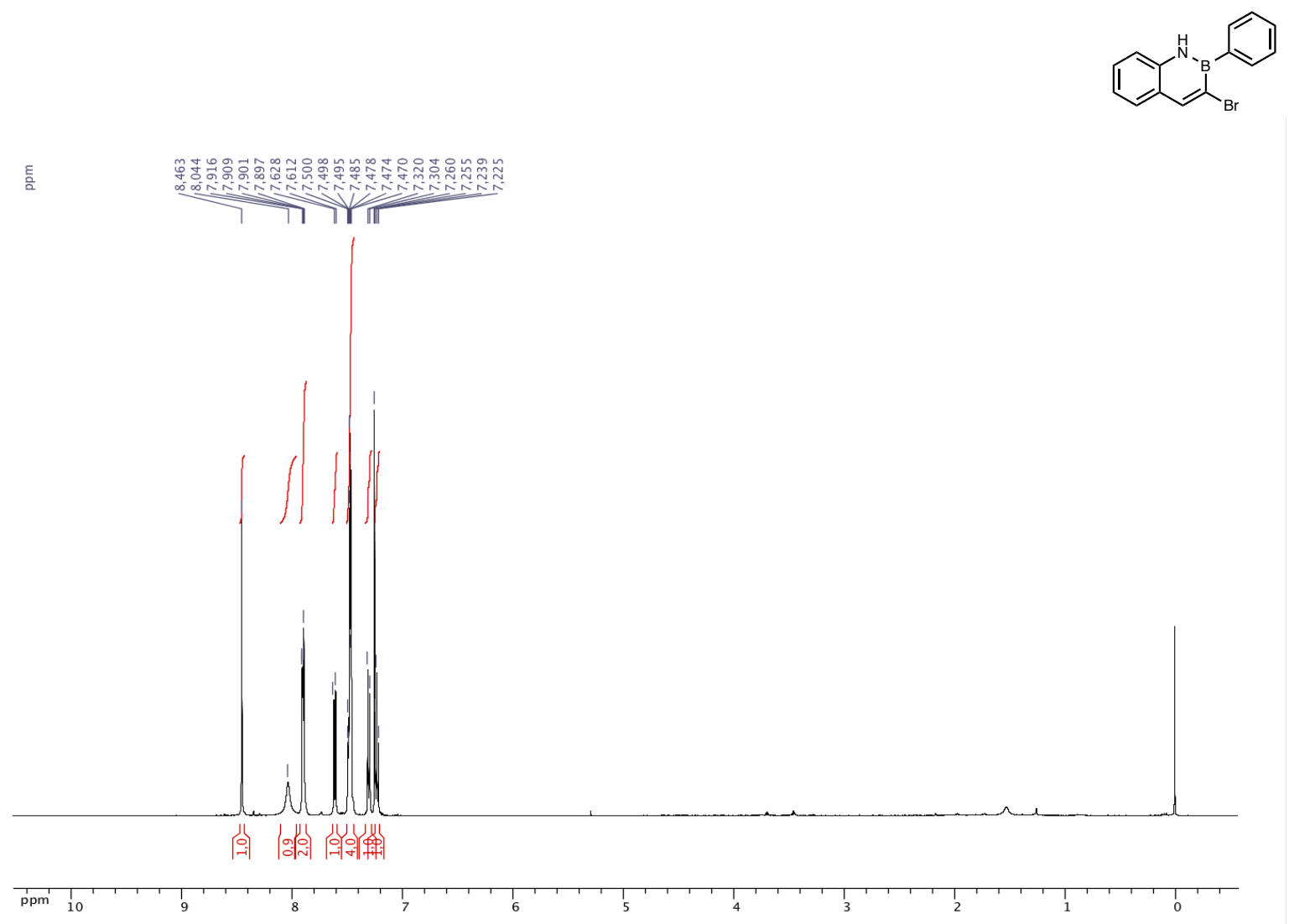

${ }^{1} \mathrm{H}$ NMR (acetone- $d_{6}, 500.4 \mathrm{MHz}$ ) of 3-bromo-2-phenyl-2,1-borazanaphthalene (1d) 

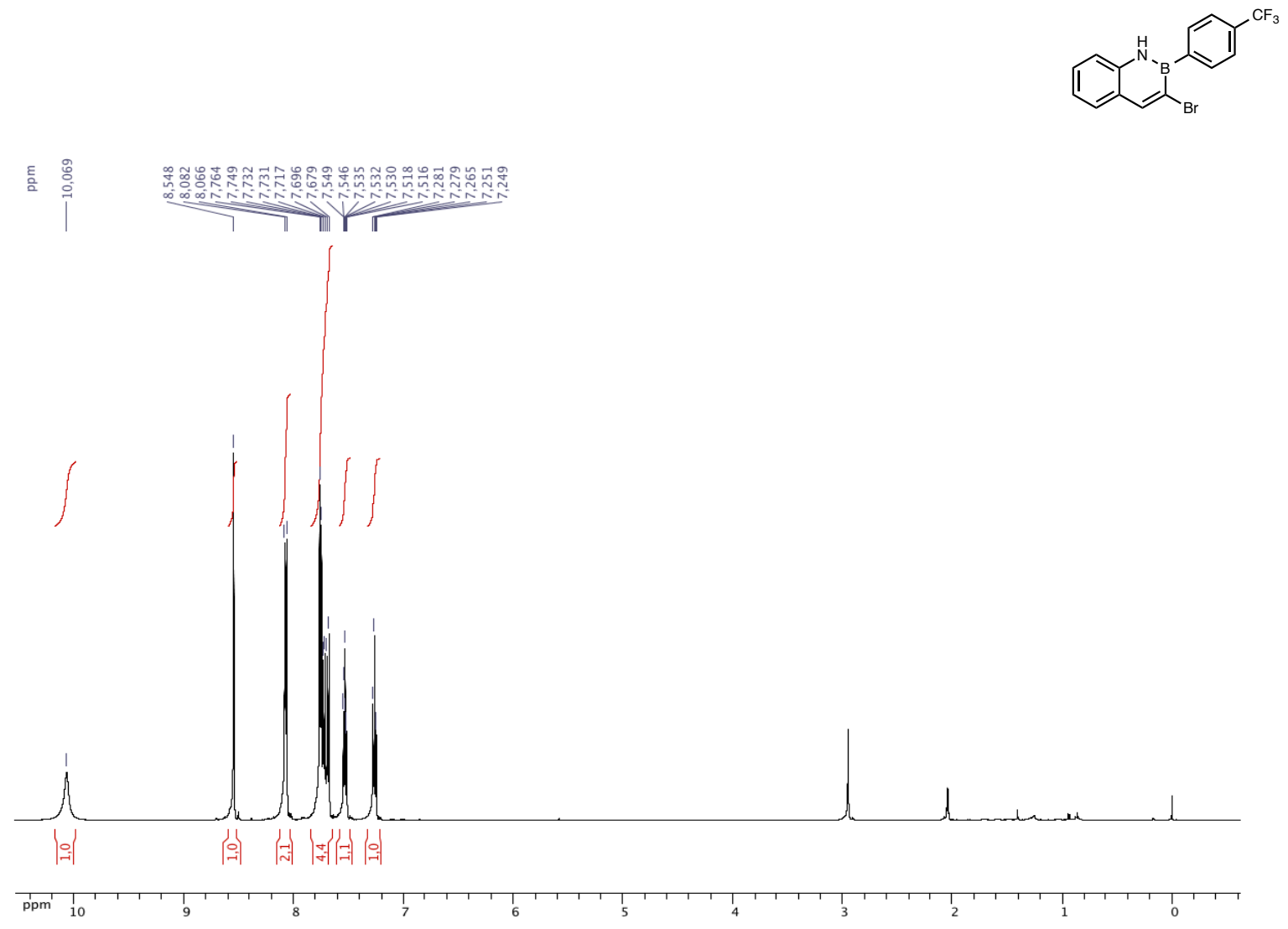

${ }^{1} \mathrm{H}$ NMR (acetone- $d_{6}, 500.4 \mathrm{MHz}$ ) of 3-bromo-2-(4-(trifluoromethyl)phenyl)-2,1-borazanaphthalene (1e)

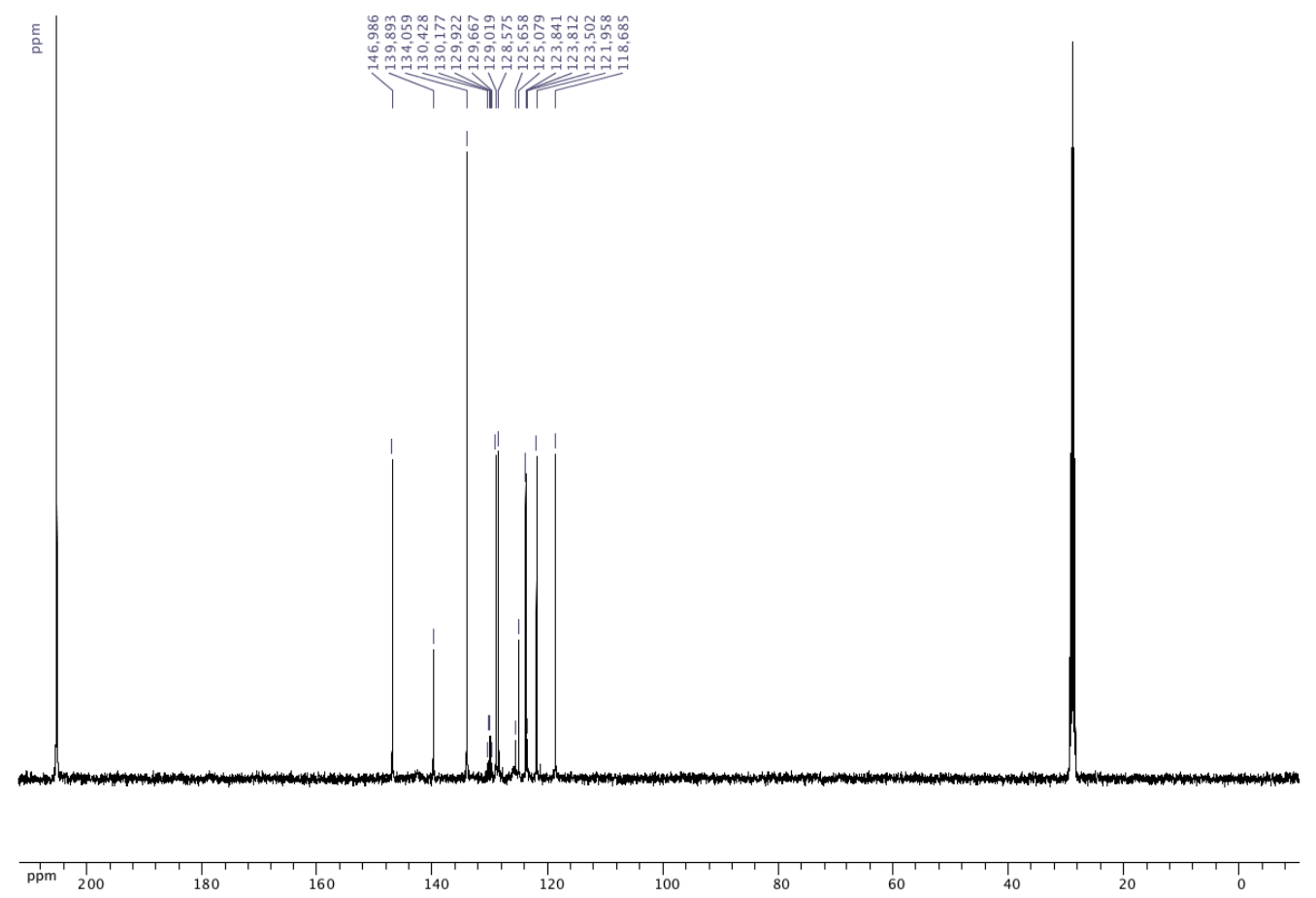

${ }^{13} \mathrm{C}\left\{{ }^{1} \mathrm{H}\right\}$ NMR (acetone- $d_{6}, 125.8 \mathrm{MHz}$ ) of 3-bromo-2-(4-(trifluoromethyl)phenyl)-2,1-borazanaphthalene (1e) 


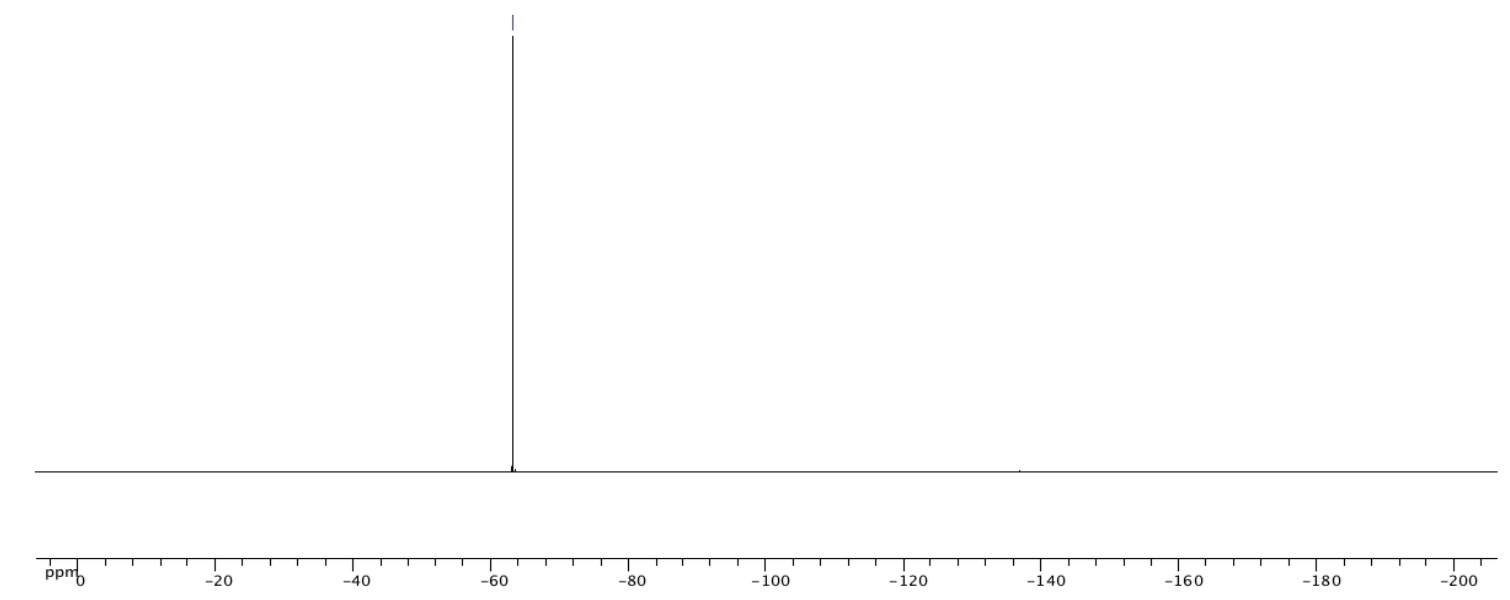

${ }^{19} \mathrm{~F}\left\{{ }^{1} \mathrm{H}\right\}$ NMR (acetone- $d_{6}, 470.8 \mathrm{MHz}$ ) of 3-bromo-2-(4-(trifluoromethyl)phenyl)-2,1-borazanaphthalene (1e) 


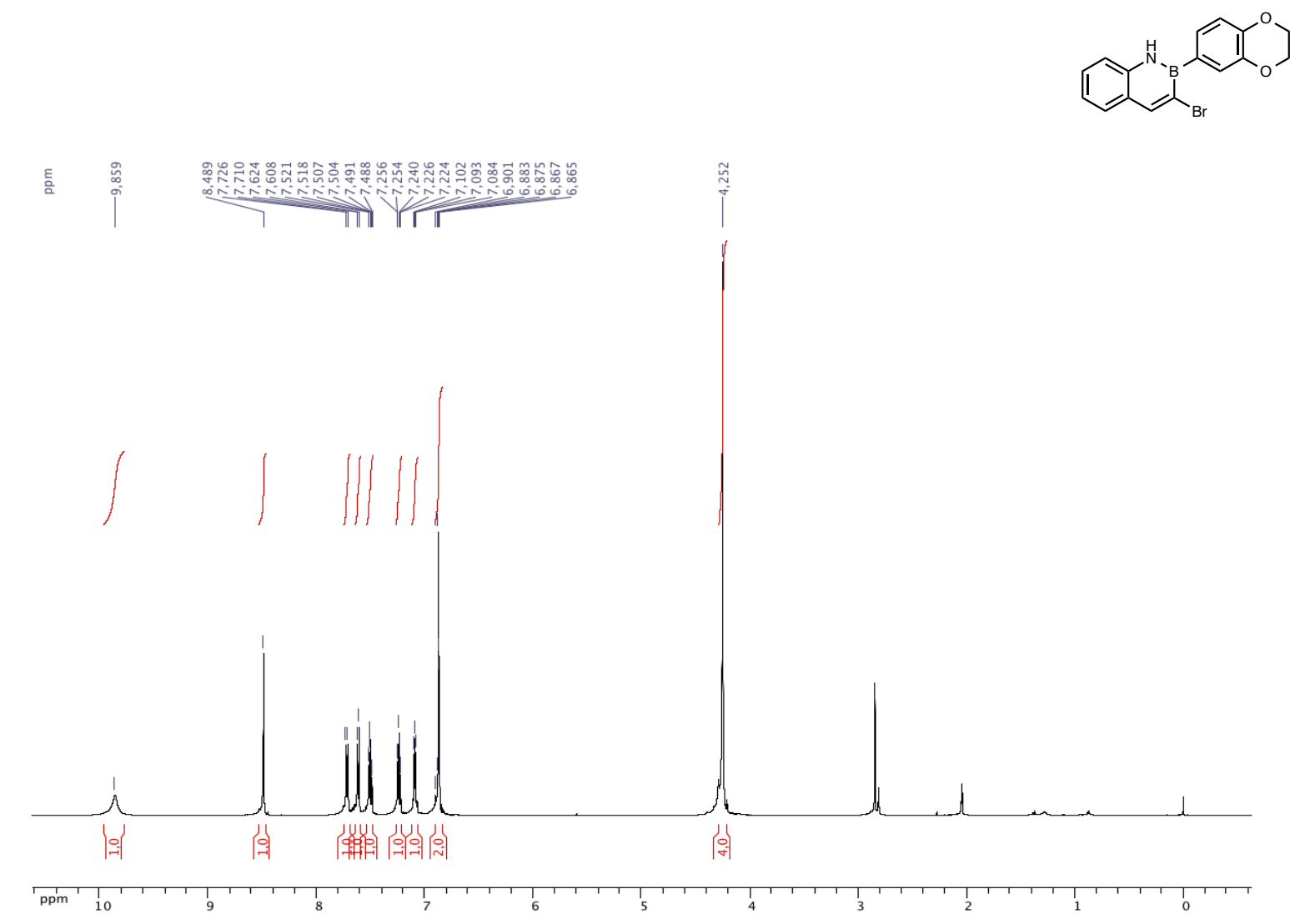

${ }^{1} \mathrm{H}$ NMR (acetone- $d_{6}, 500.4 \mathrm{MHz}$ ) of 3-bromo-2-(2,3-dihydro-1,4-benzodioxin-6-yl)-2,1-borazanaphthalene (1f)
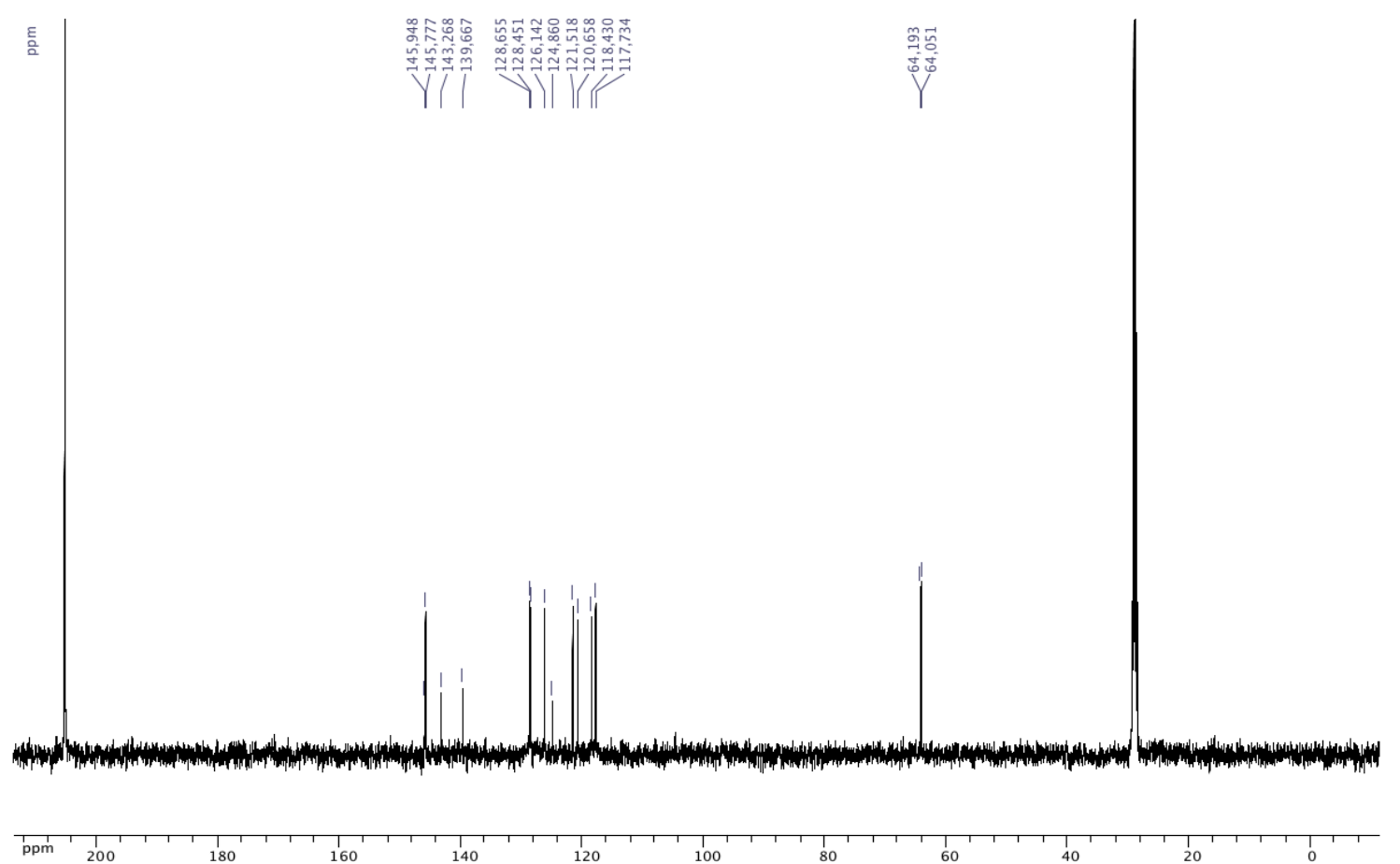

${ }^{13} \mathrm{C}\left\{{ }^{1} \mathrm{H}\right\}$ NMR (acetone- $d_{6}, 125.8 \mathrm{MHz}$ ) of 3-bromo-2-(2,3-dihydro-1,4-benzodioxin-6-yl)-2,1borazanaphthalene (1f) 

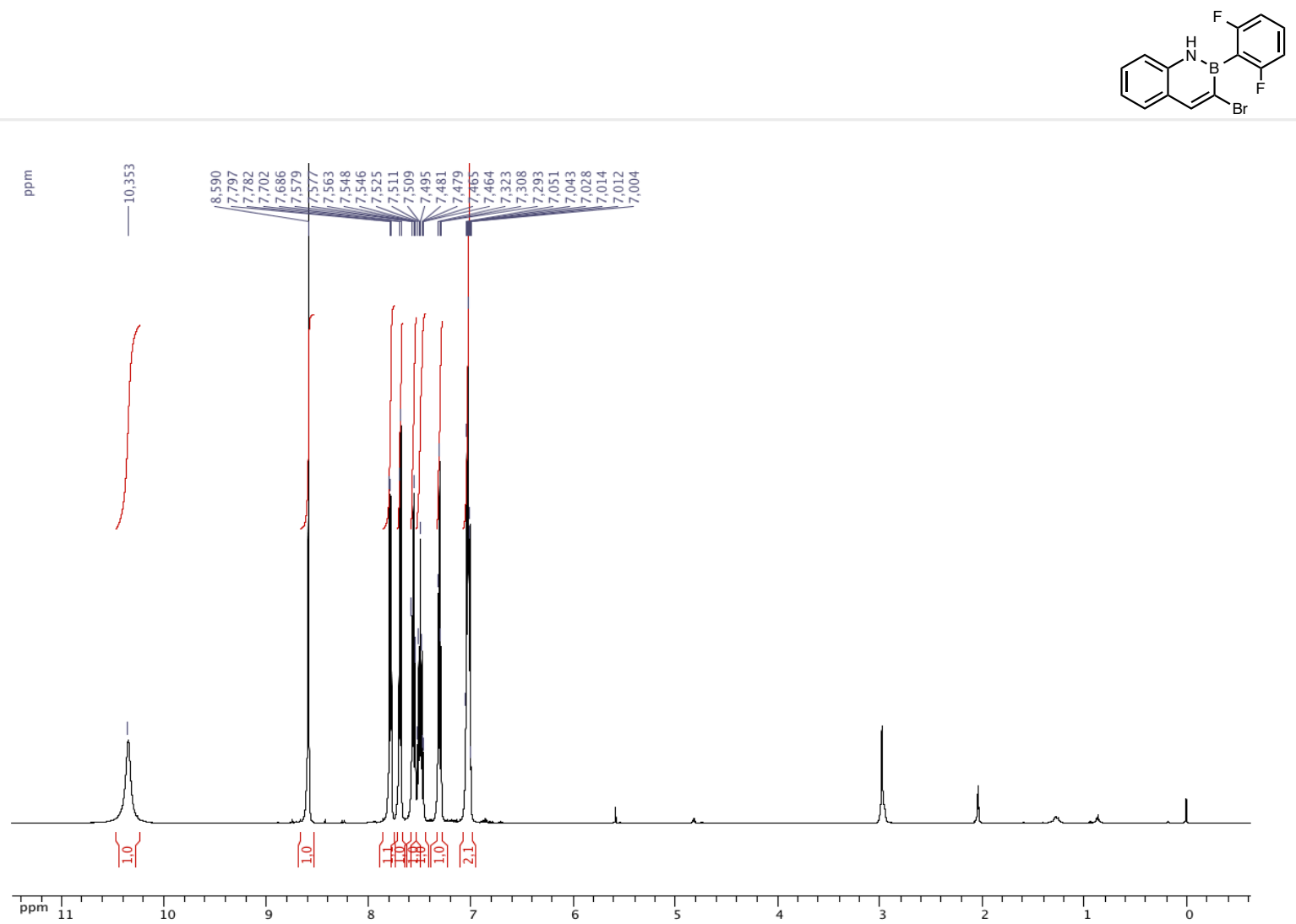

${ }^{1} \mathrm{H}$ NMR (acetone- $d_{6}, 500.4 \mathrm{MHz}$ ) of 3-bromo-2-(2,6-difluorophenyl)-2,1-borazanaphthalene (1g)
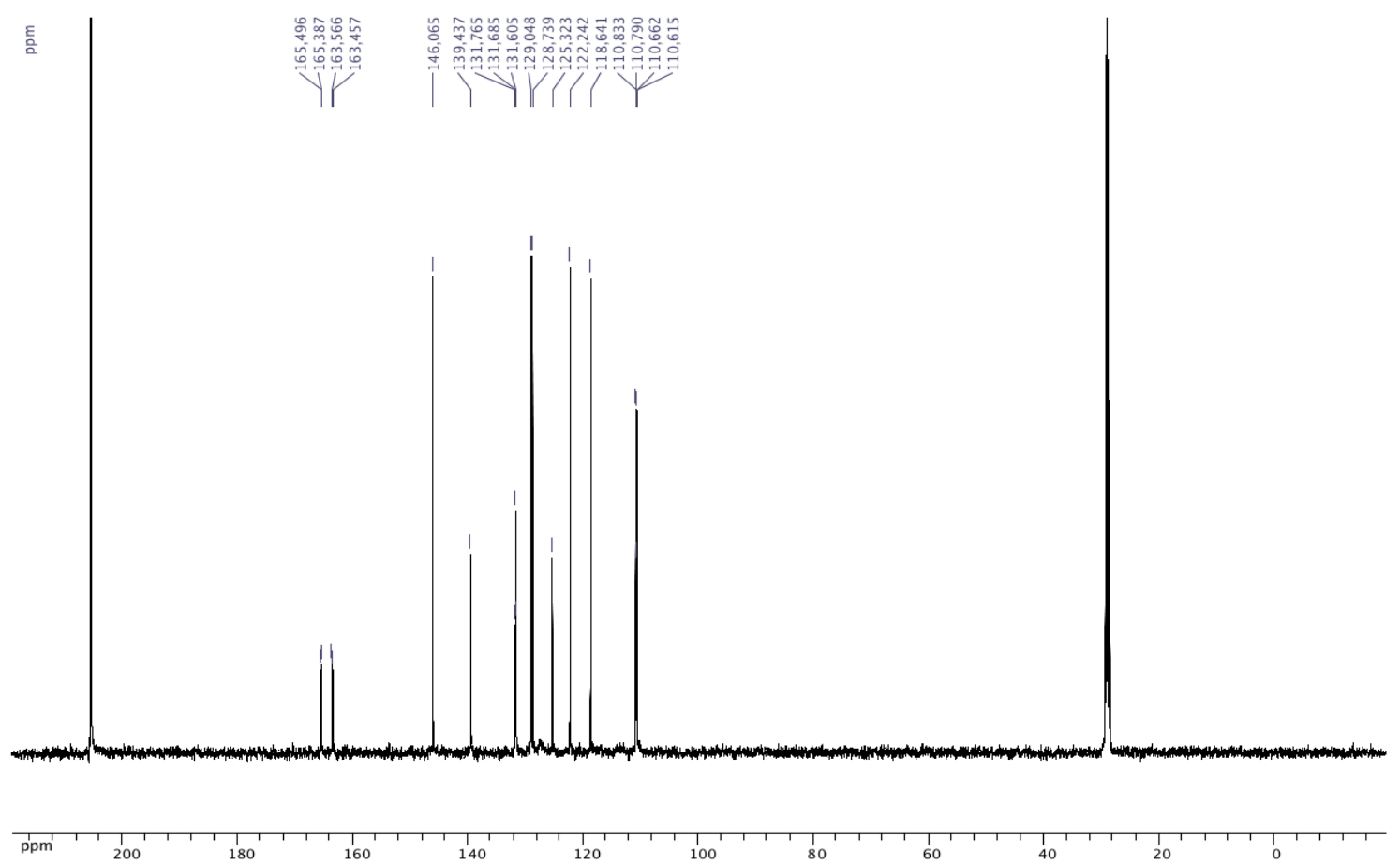

${ }^{13} \mathrm{C}\left\{{ }^{1} \mathrm{H}\right\}$ NMR (acetone- $d_{6}, 125.8 \mathrm{MHz}$ ) of 3-bromo-2-(2,6-difluorophenyl)-2,1-borazanaphthalene (19) 


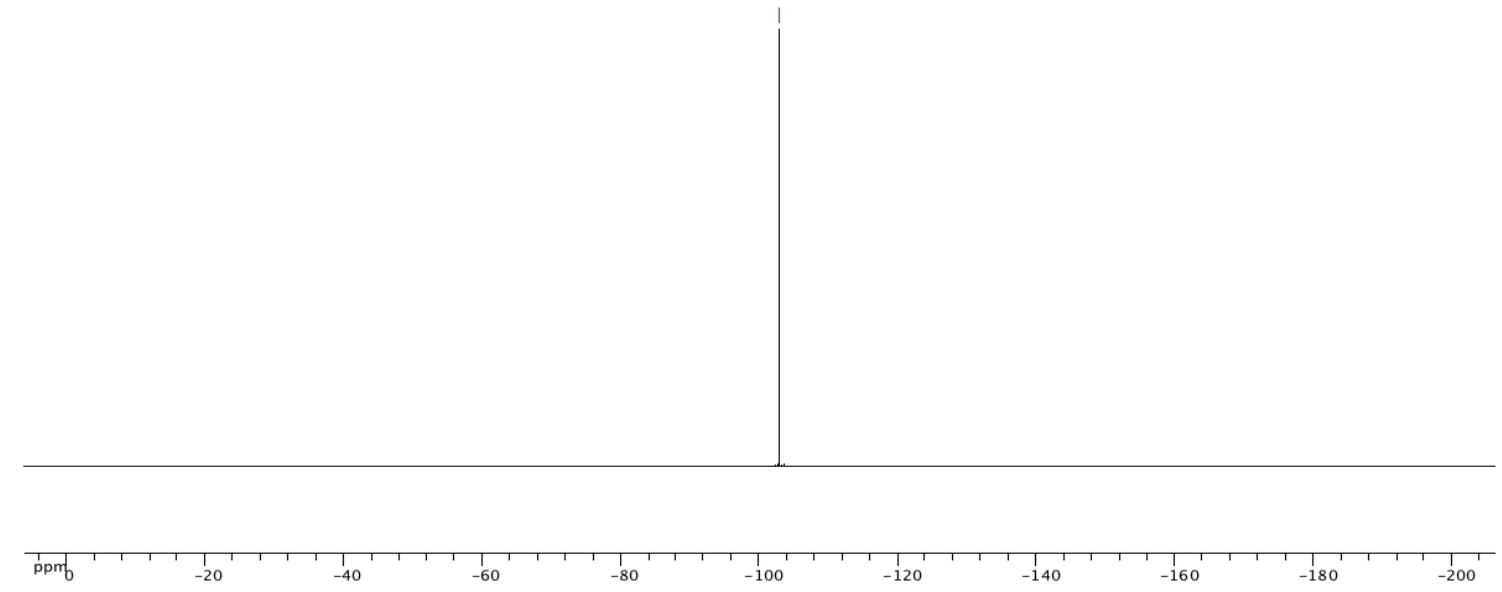

${ }^{19} \mathrm{~F}\left\{{ }^{1} \mathrm{H}\right\}$ NMR (acetone- $d_{6}, 470.8 \mathrm{MHz}$ ) of 3-bromo-2-(2,6-difluorophenyl)-2,1-borazanaphthalene (1g) 


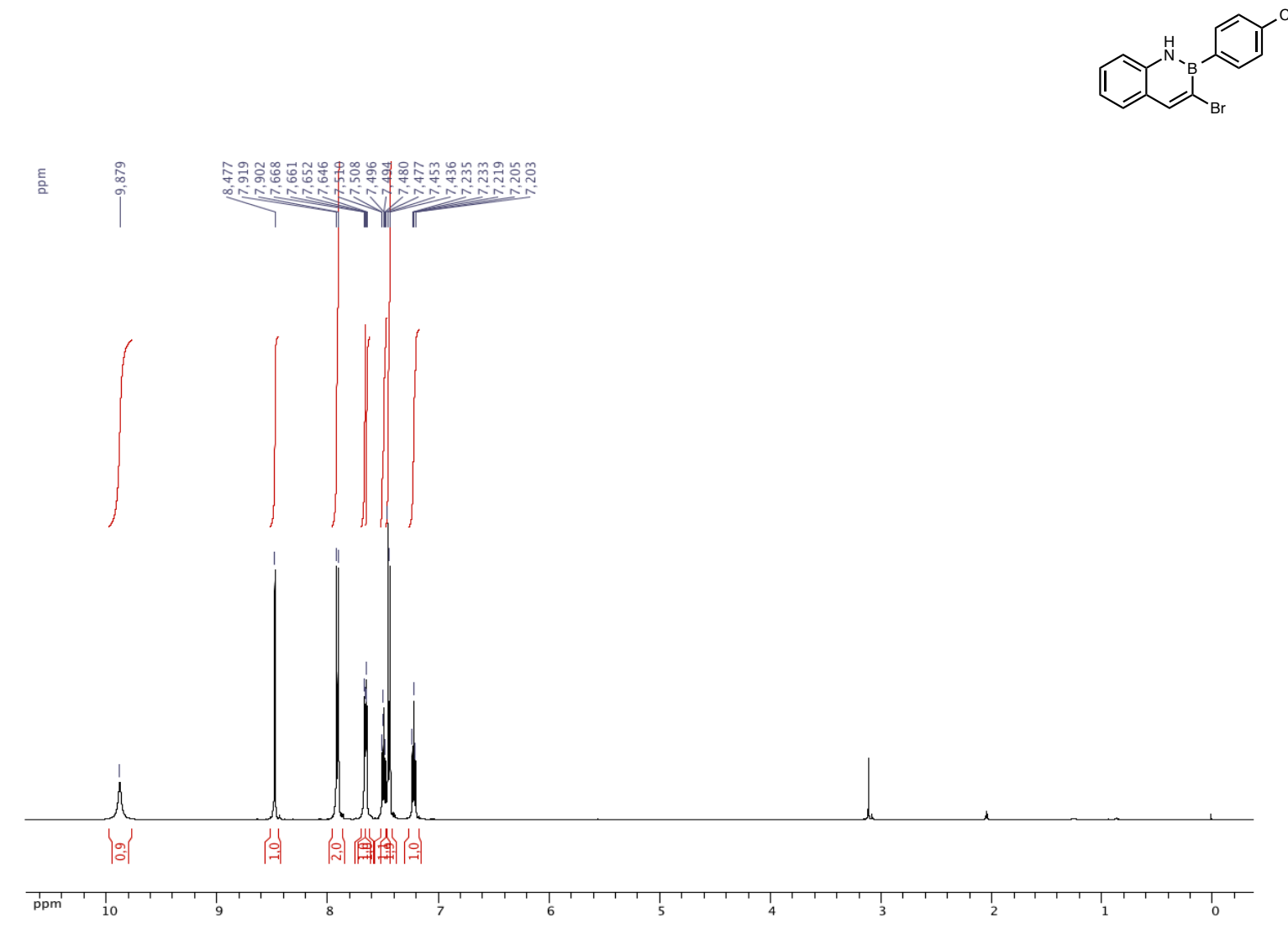

${ }^{1} \mathrm{H}$ NMR (acetone- $d_{6}, 500.4 \mathrm{MHz}$ ) of 3-bromo-2-(4-chlorophenyl)-2,1-borazanaphthalene (1h)
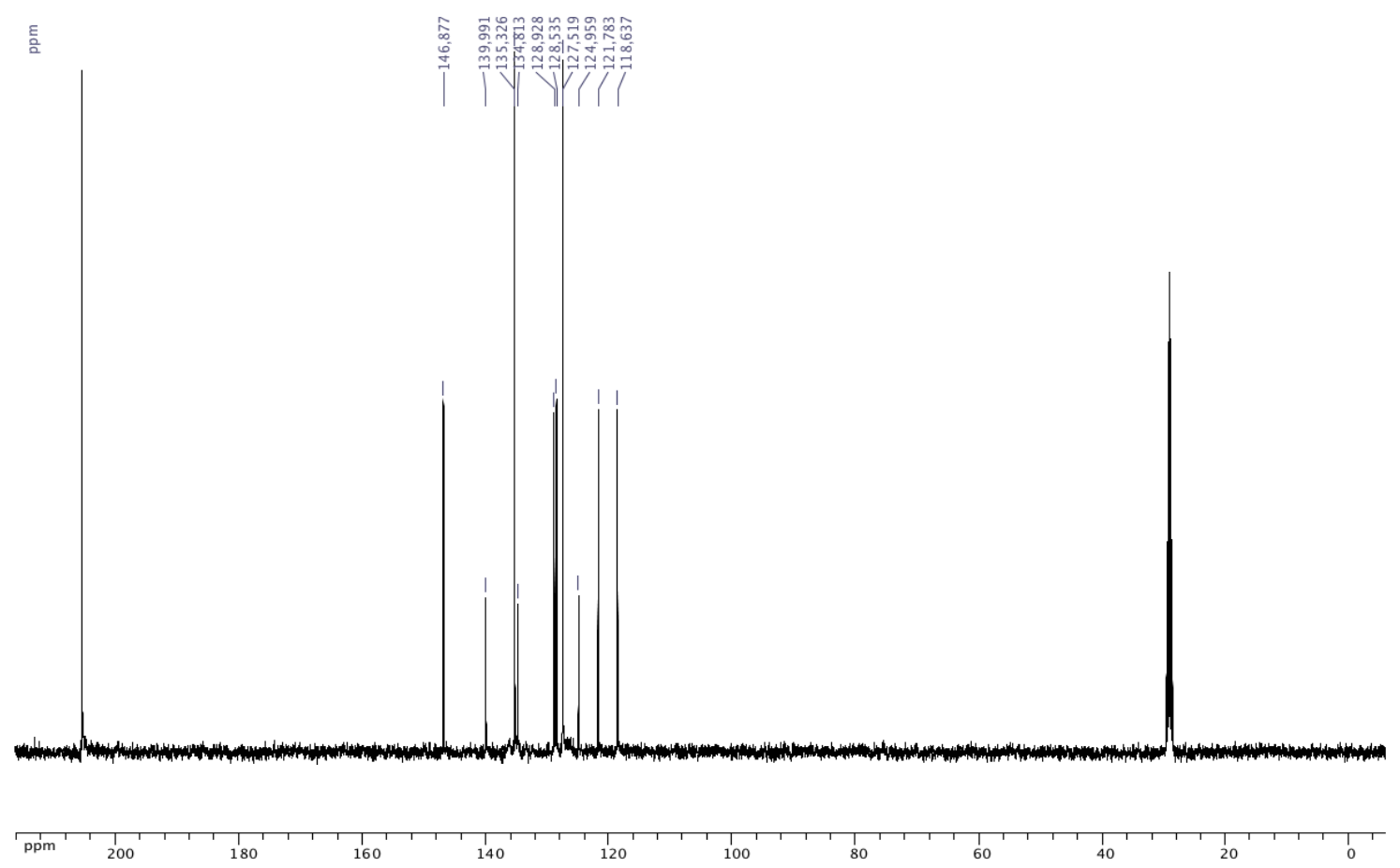

${ }^{13} \mathrm{C}\left\{{ }^{1} \mathrm{H}\right\}$ NMR (acetone- $d_{6}, 125.8 \mathrm{MHz}$ ) of 3-bromo-2-(4-chlorophenyl)-2,1-borazanaphthalene (1h) 


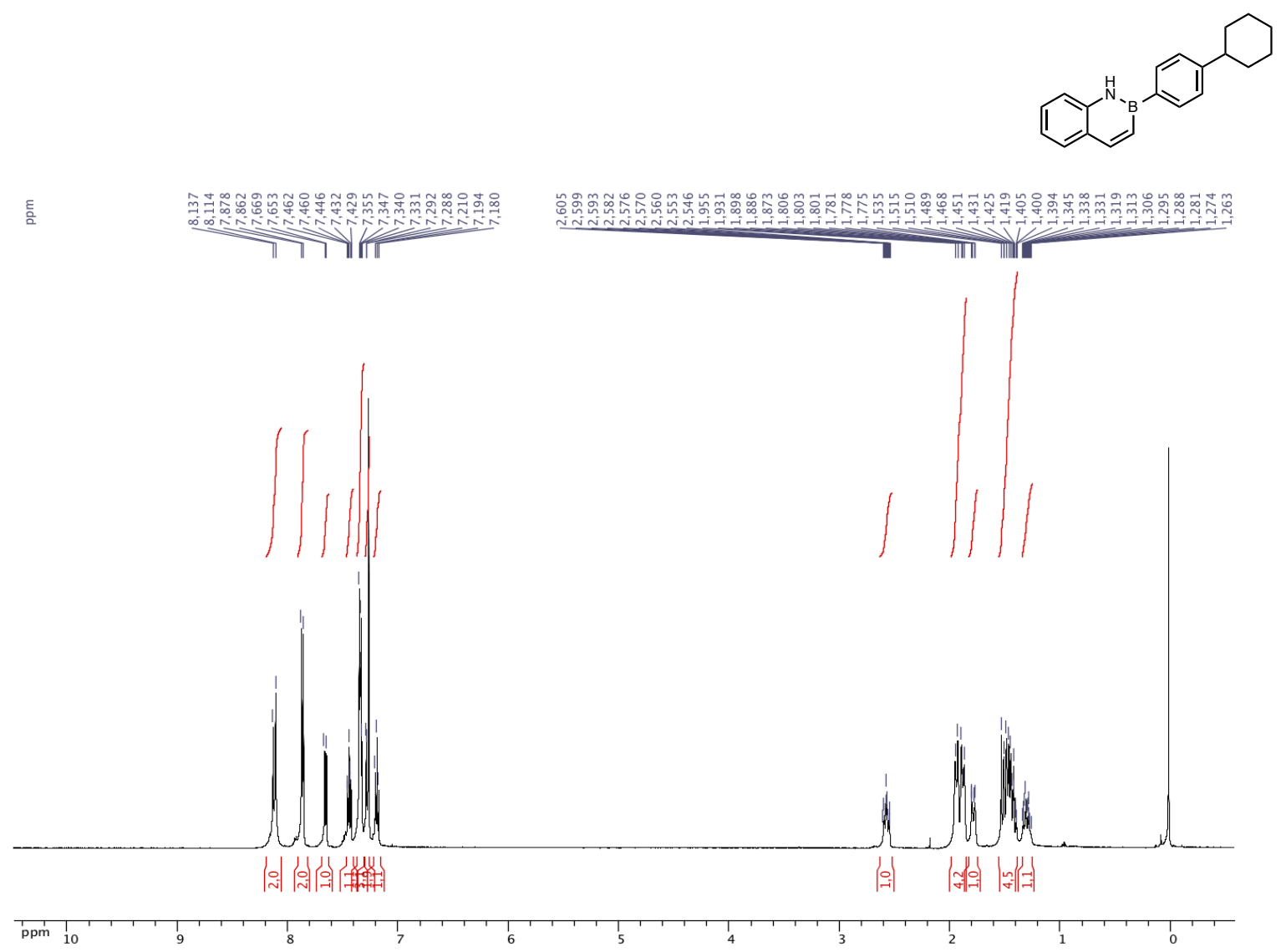

$\left.{ }^{1} \mathrm{H} \mathrm{NMR} \mathrm{(CDCl} 3,500.4 \mathrm{MHz}\right)$ of 2-(4-cyclohexylphenyl)-2,1-borazanaphthalene (2a)
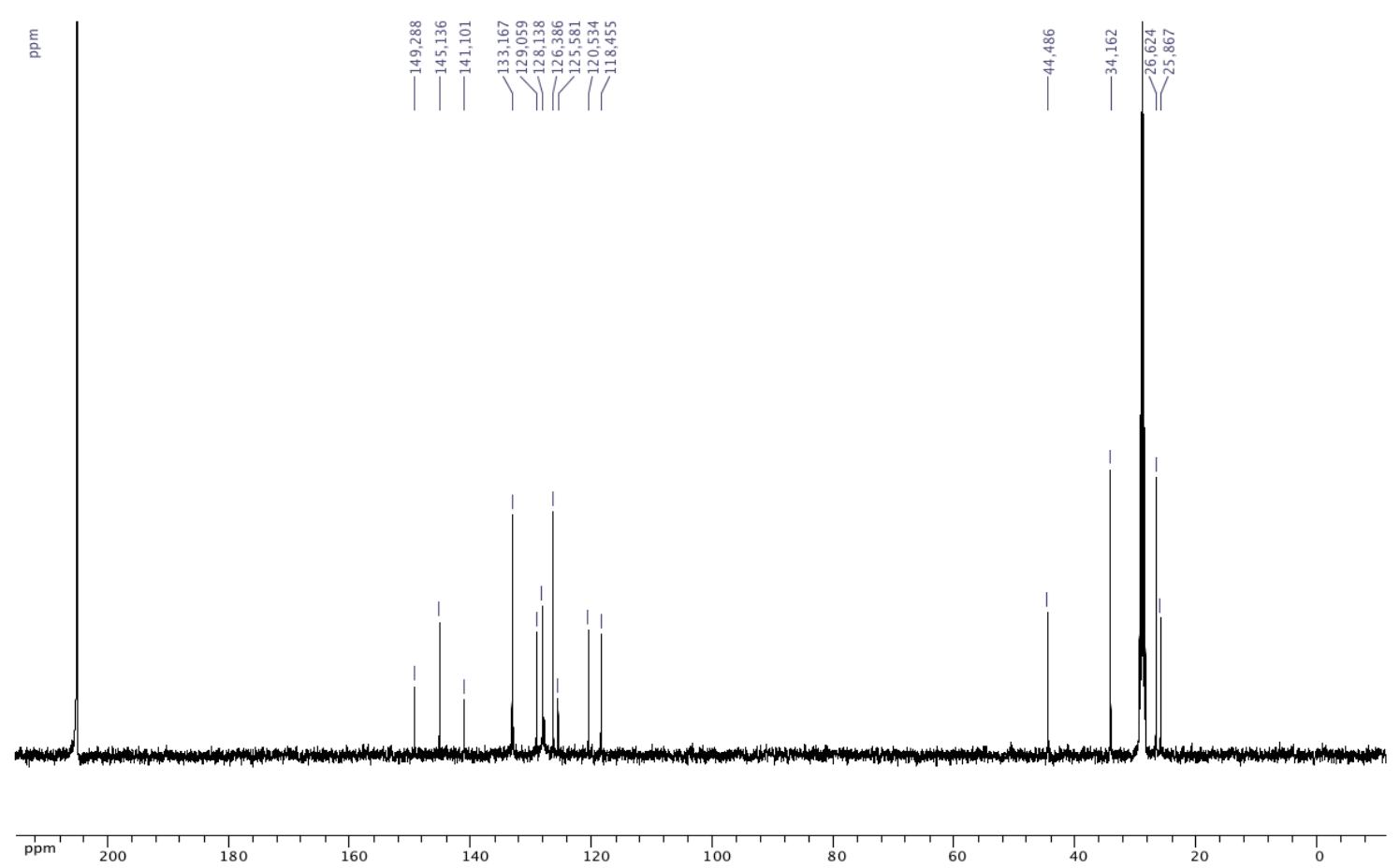

${ }^{13} \mathrm{C}\left\{{ }^{1} \mathrm{H}\right\}$ NMR $\left(\mathrm{CDCl}_{3}, 125.8 \mathrm{MHz}\right)$ of 2-(4-cyclohexylphenyl)-2,1-borazanaphthalene (2a) 


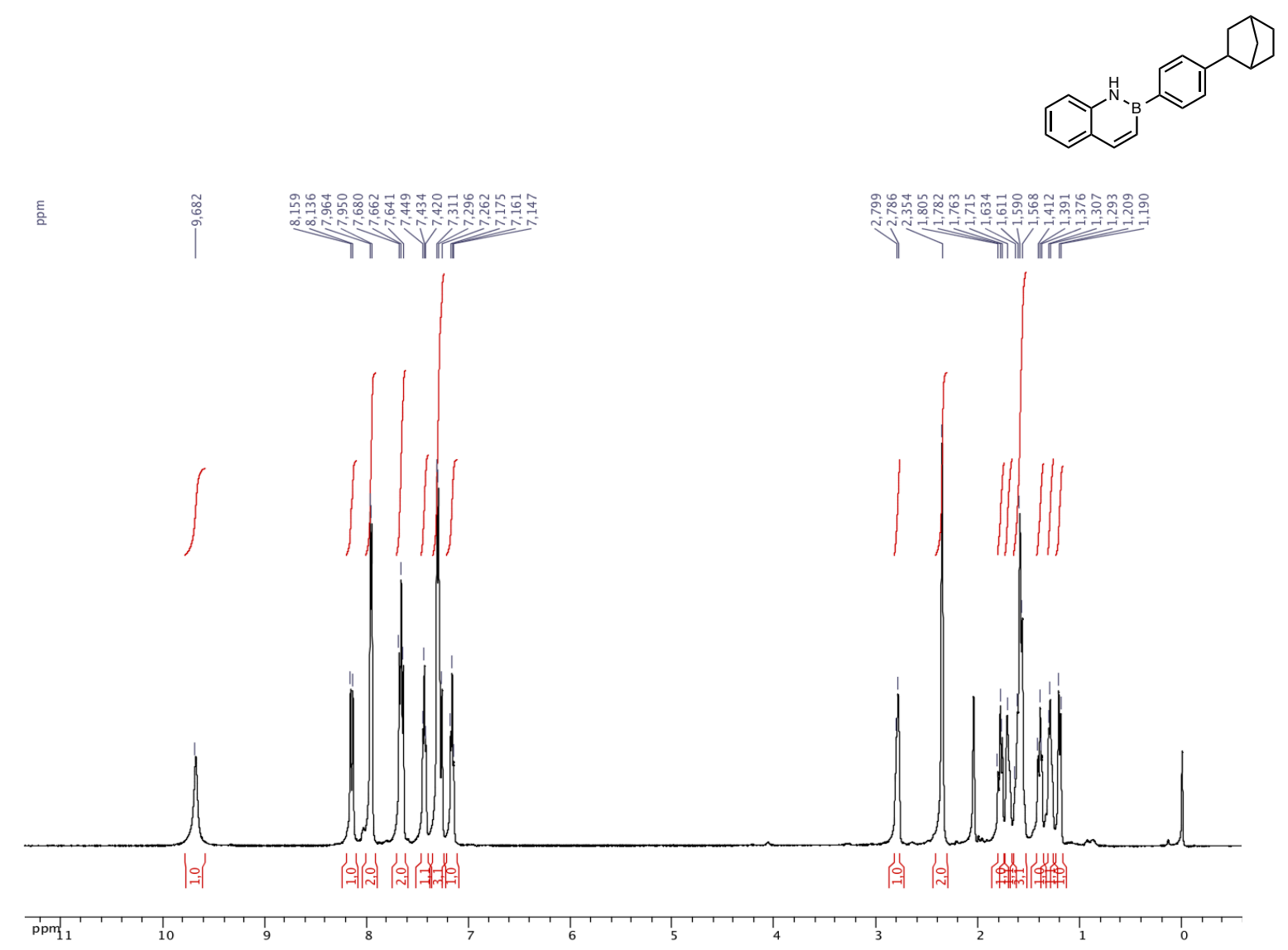

${ }^{1} \mathrm{H}$ NMR (acetone- $d_{6}, 500.4 \mathrm{MHz}$ ) of 2-(4-(exo-bicyclo[2.2.1]heptan-2-yl)phenyl)-2,1-borazanaphthalene (2b)
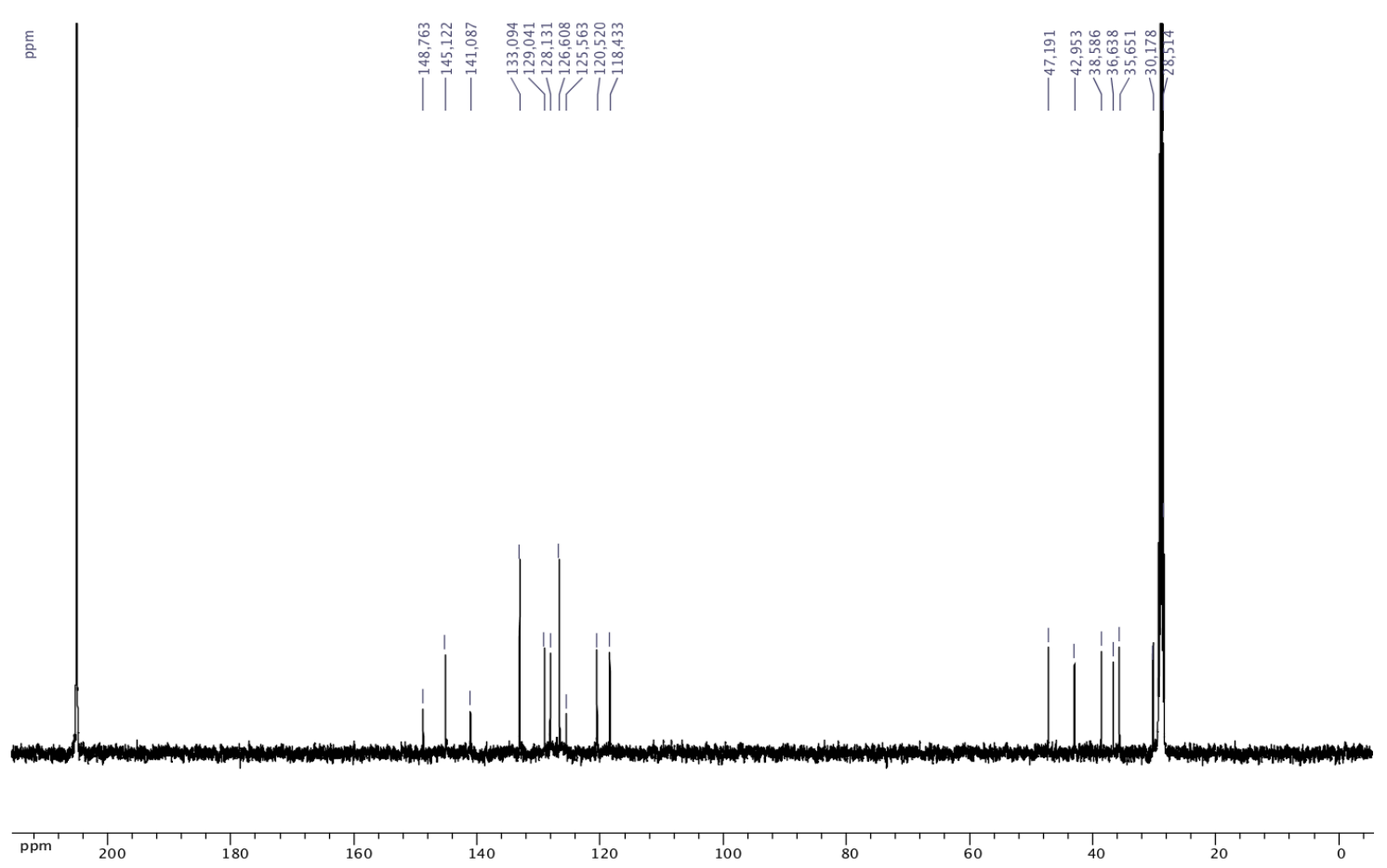

${ }^{13} \mathrm{C}\left\{{ }^{1} \mathrm{H}\right\}$ NMR (acetone- $d_{6}, 125.8 \mathrm{MHz}$ ) of 2-(4-(exo-bicyclo[2.2.1]heptan-2-yl)phenyl)-2,1-borazanaphthalene (2b) 


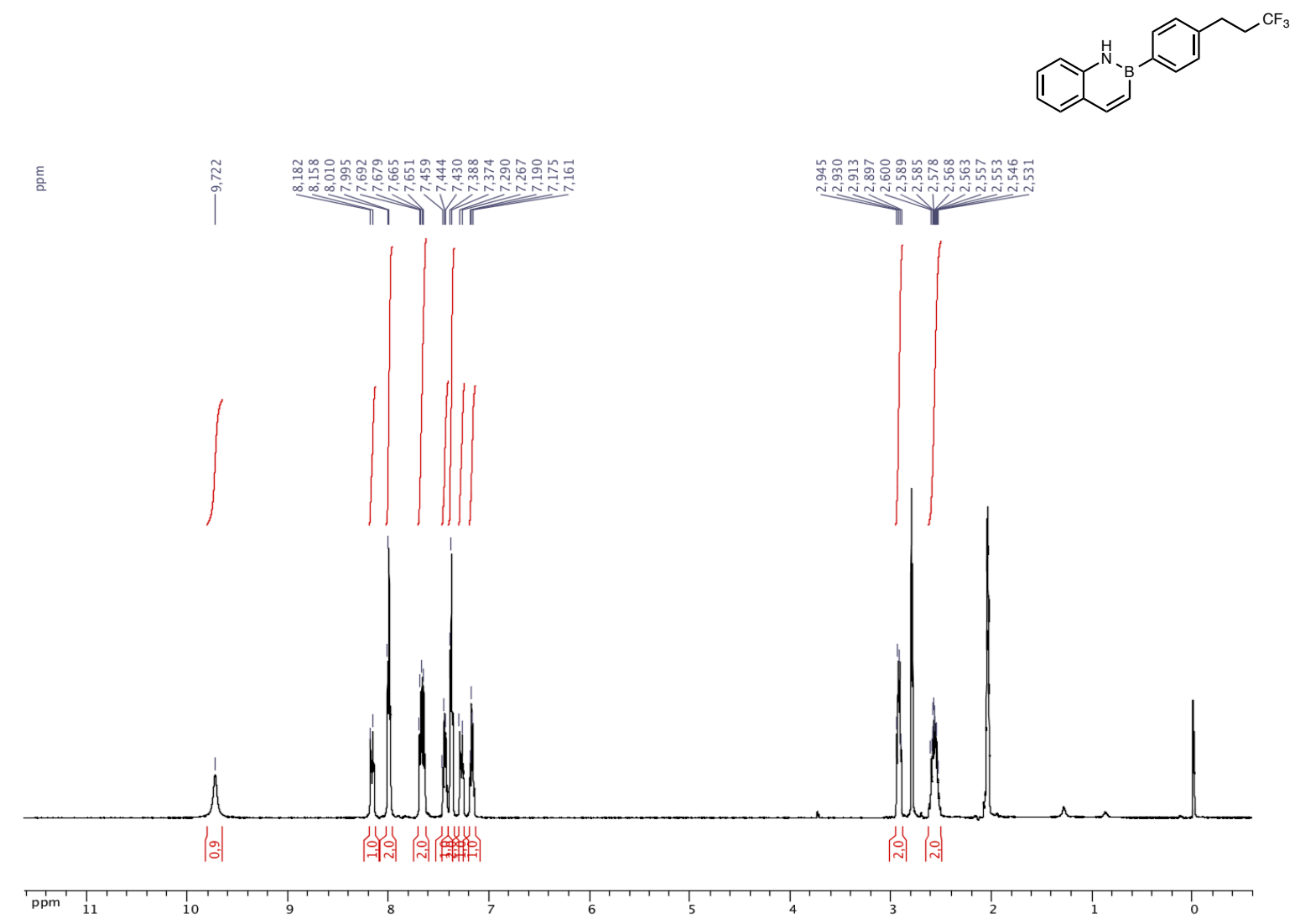

${ }^{1} \mathrm{H}$ NMR (acetone- $d_{6}, 500.4 \mathrm{MHz}$ ) of 2-(4-(3,3,3-trifluoropropyl)phenyl)-2,1-borazanaphthalene (2c) 言
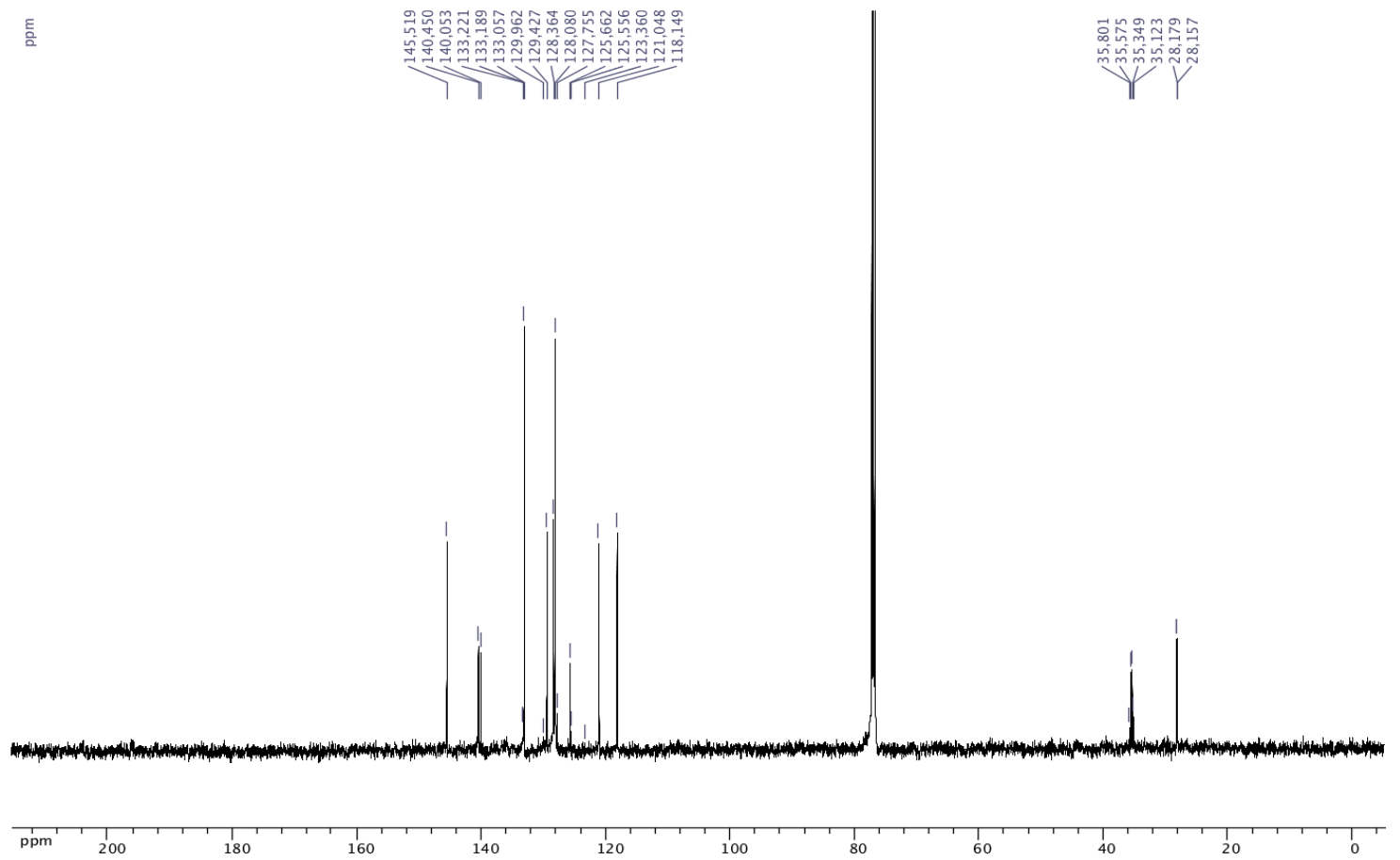

${ }^{13} \mathrm{C}\left\{{ }^{1} \mathrm{H}\right\}\left(\mathrm{CDCl}_{3}, 125.8 \mathrm{MHz}\right)$ of 2-(4-(3,3,3-trifluoropropyl)phenyl)-2,1-borazanaphthalene (2c) 


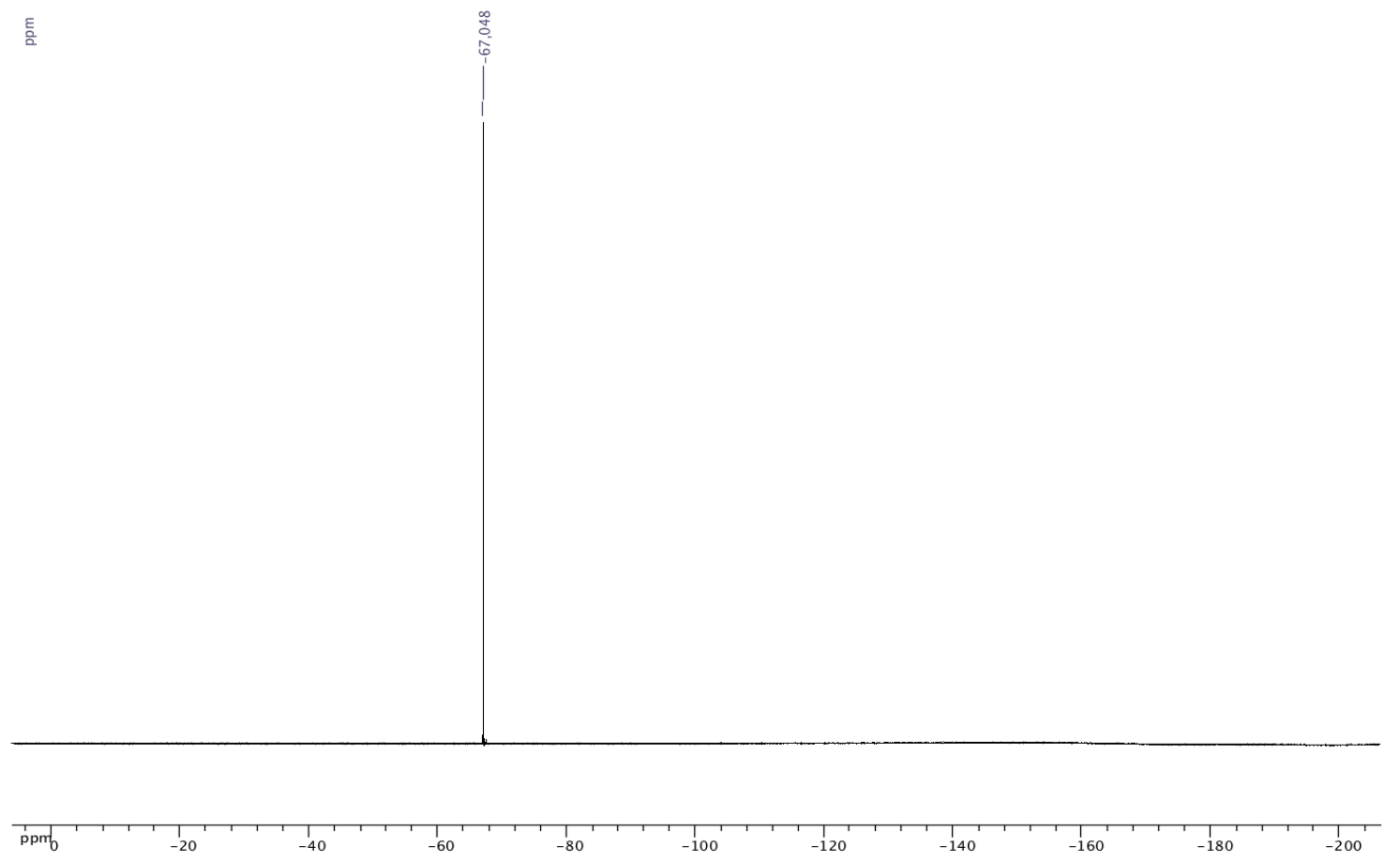

${ }^{19} \mathrm{~F}\left\{{ }^{1} \mathrm{H}\right\}$ NMR (acetone- $d_{6}, 470.8 \mathrm{MHz}$ ) of 2-(4-(3,3,3-trifluoropropyl)phenyl)-2,1-borazanaphthalene (2c) 


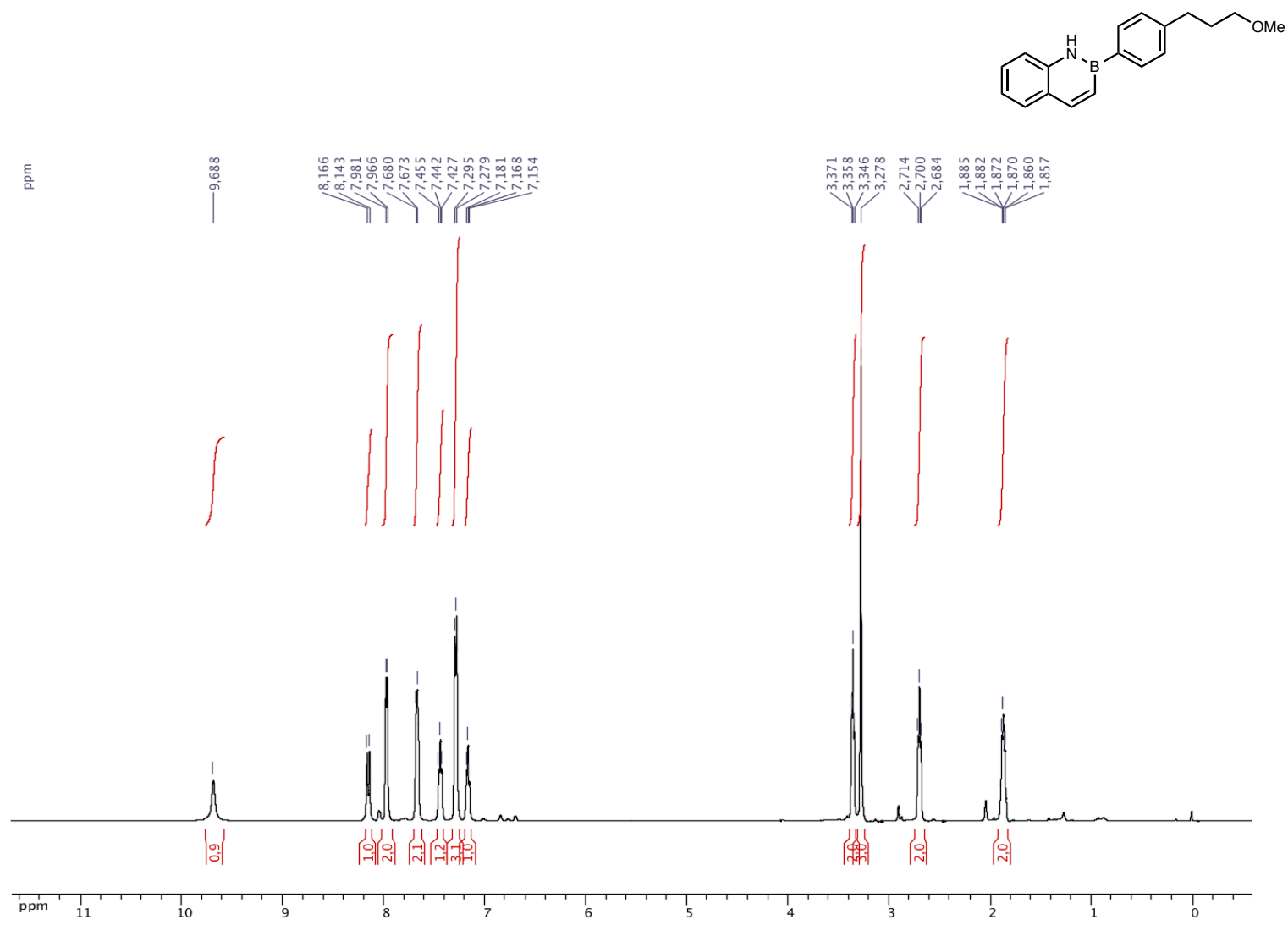

${ }^{1} \mathrm{H}$ NMR (acetone- $d_{6}, 500.4 \mathrm{MHz}$ ) of 2-(4-(3-methoxypropyl)phenyl)-2,1-borazanaphthalene (2d)
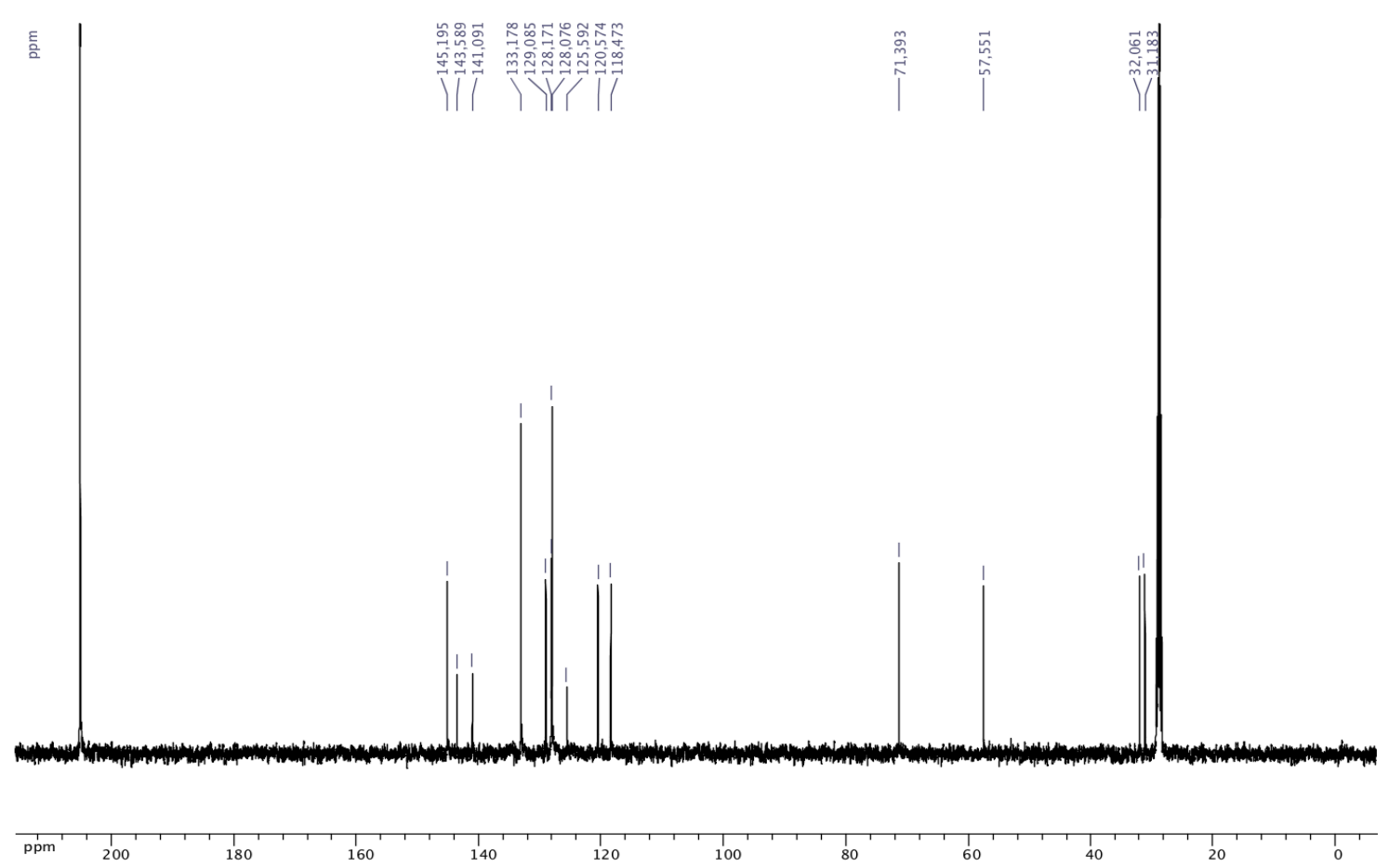

${ }^{13} \mathrm{C}\left\{{ }^{1} \mathrm{H}\right\}$ (acetone- $d_{6}, 125.8 \mathrm{MHz}$ ) of 2-(4-(3-methoxypropyl)phenyl)-2,1-borazanaphthalene (2d) 


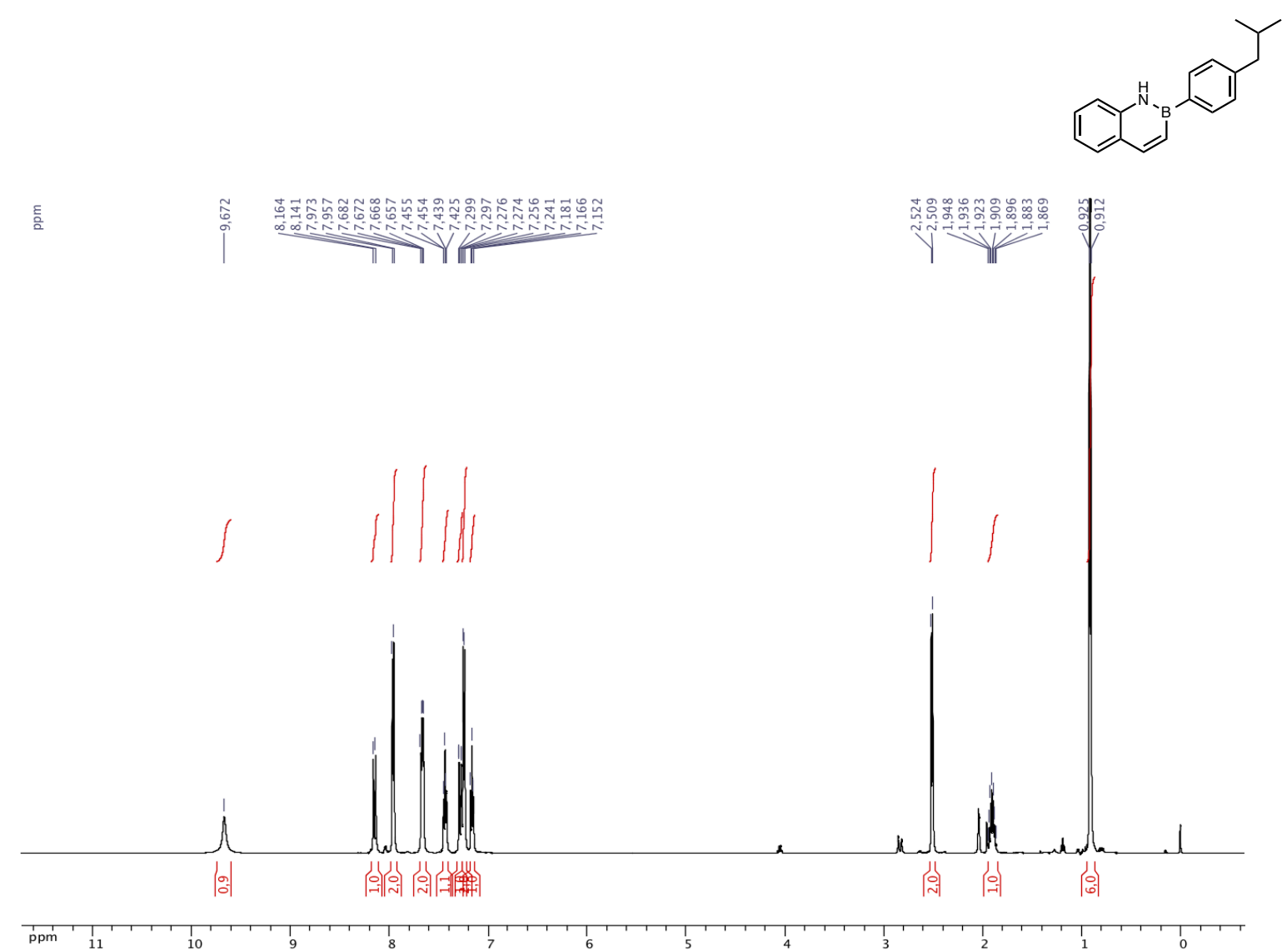

${ }^{1} \mathrm{H}$ NMR (acetone- $d_{6}, 500.4 \mathrm{MHz}$ ) of 2-(4-isobutylphenyl)-2,1-borazanaphthalene (2e)
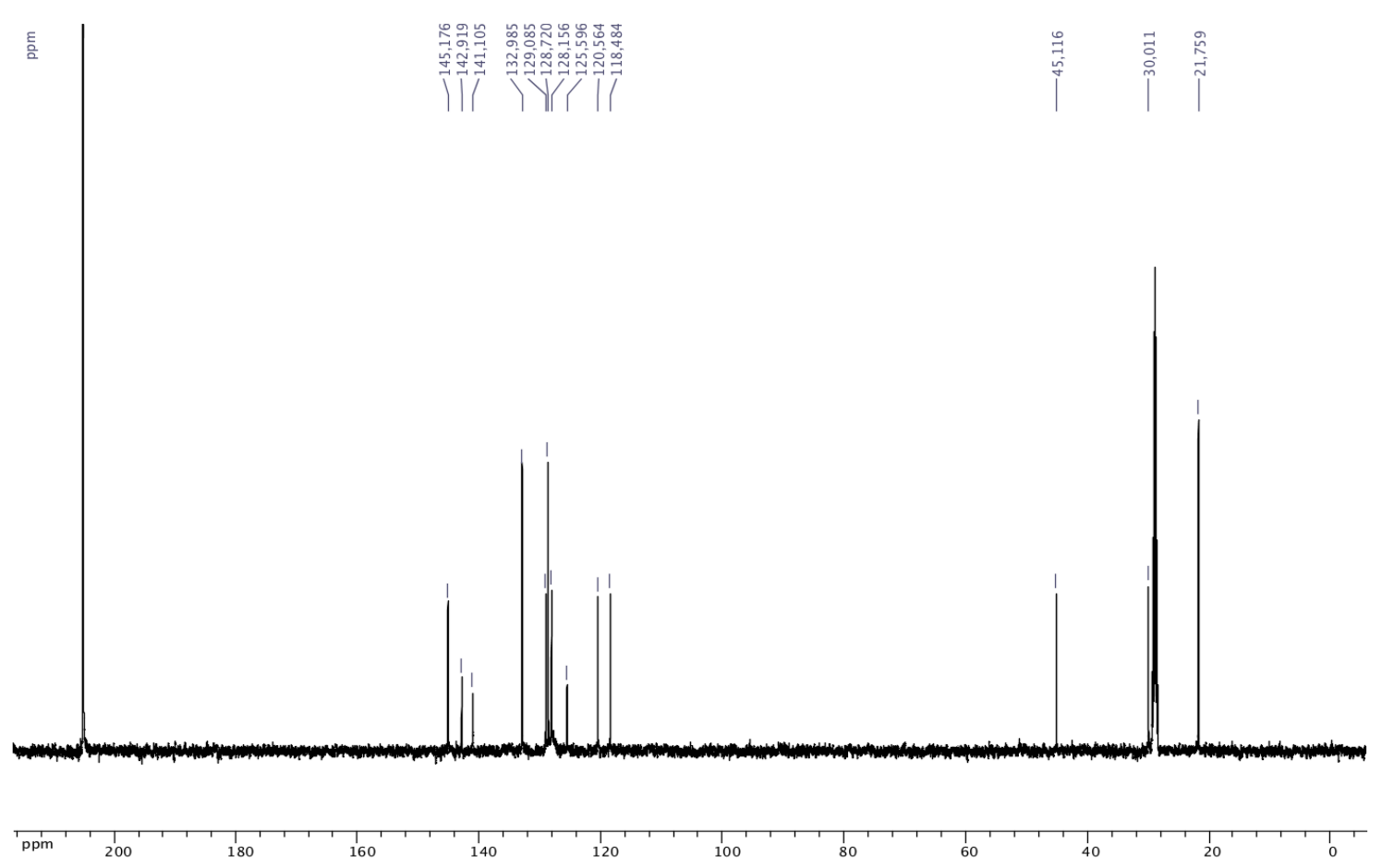

${ }^{13} \mathrm{C}\left\{{ }^{1} \mathrm{H}\right\}$ NMR (acetone- $\left.d_{6}, 125.8 \mathrm{MHz}\right)$ of 2-(4-isobutylphenyl)-2,1-borazanaphthalene (2e) 


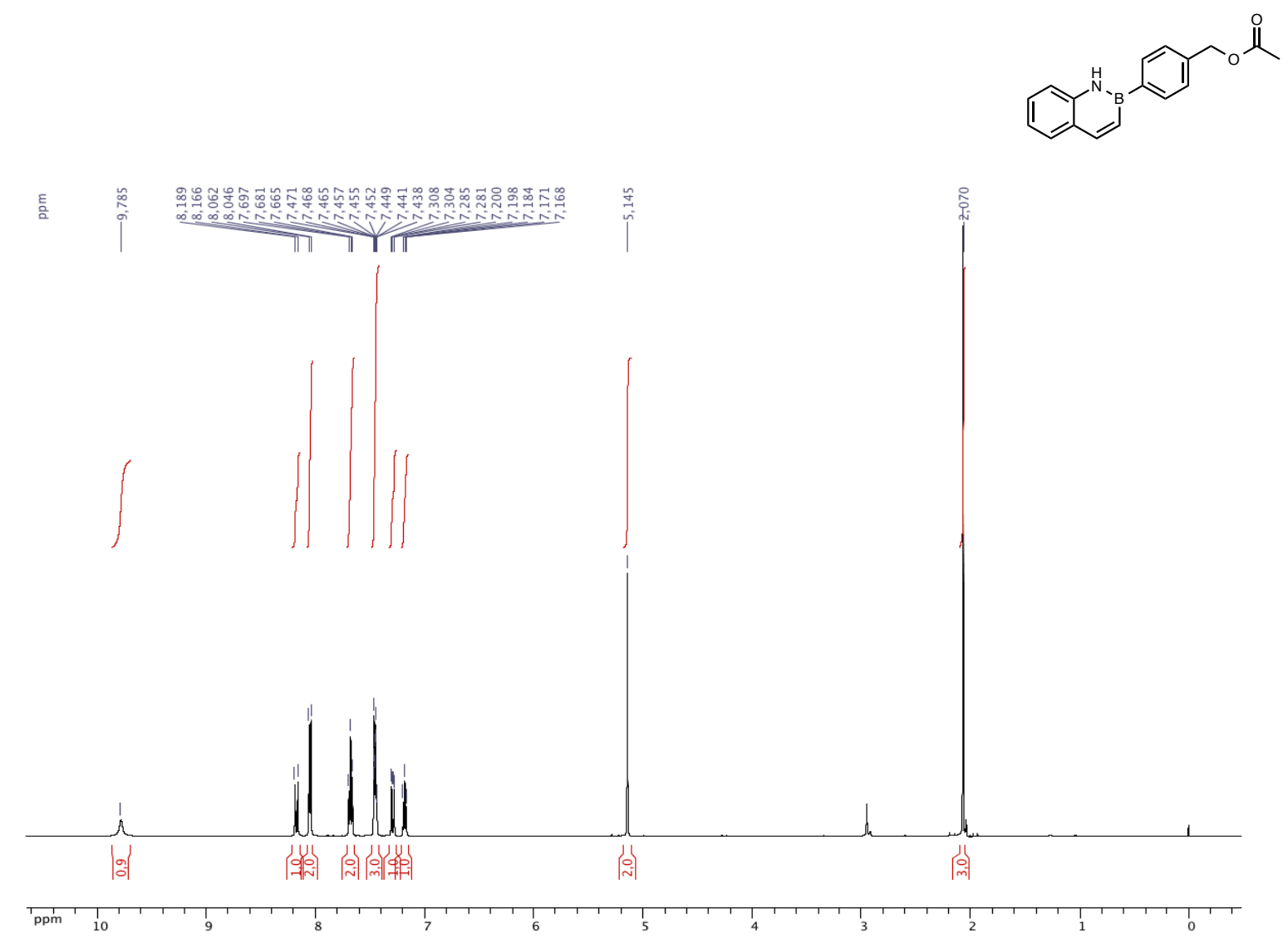

${ }^{1} \mathrm{H}$ NMR (acetone- $d_{6}, 500.4 \mathrm{MHz}$ ) of 2-(4-(acetoxymethyl)phenyl)-2,1-borazanaphthalene (2f)
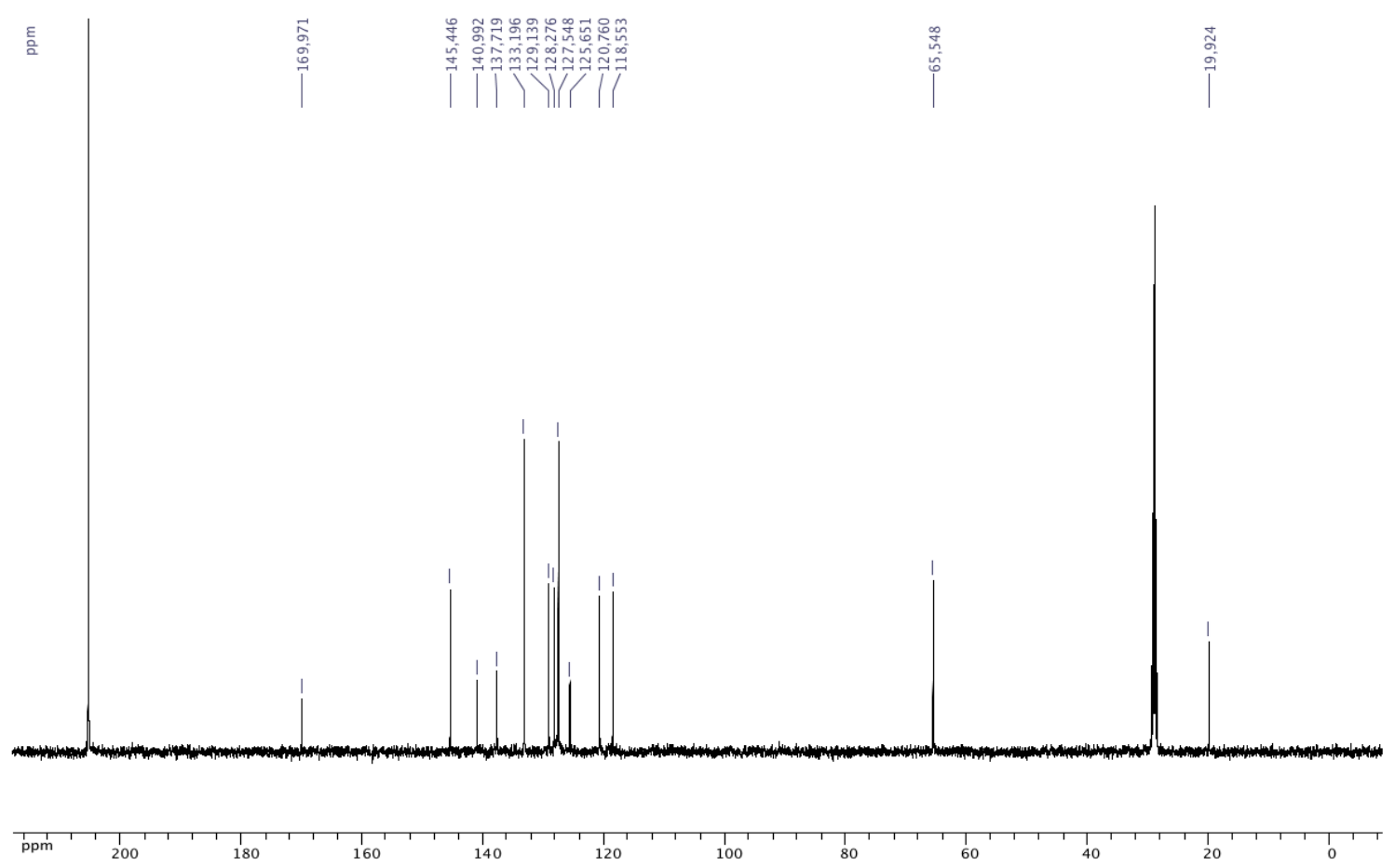

${ }^{13} \mathrm{C}\left\{{ }^{1} \mathrm{H}\right\}$ NMR (acetone- $d_{6}, 125.8 \mathrm{MHz}$ ) of 2-(4-(acetoxymethyl)phenyl)-2,1-borazanaphthalene (2f) 


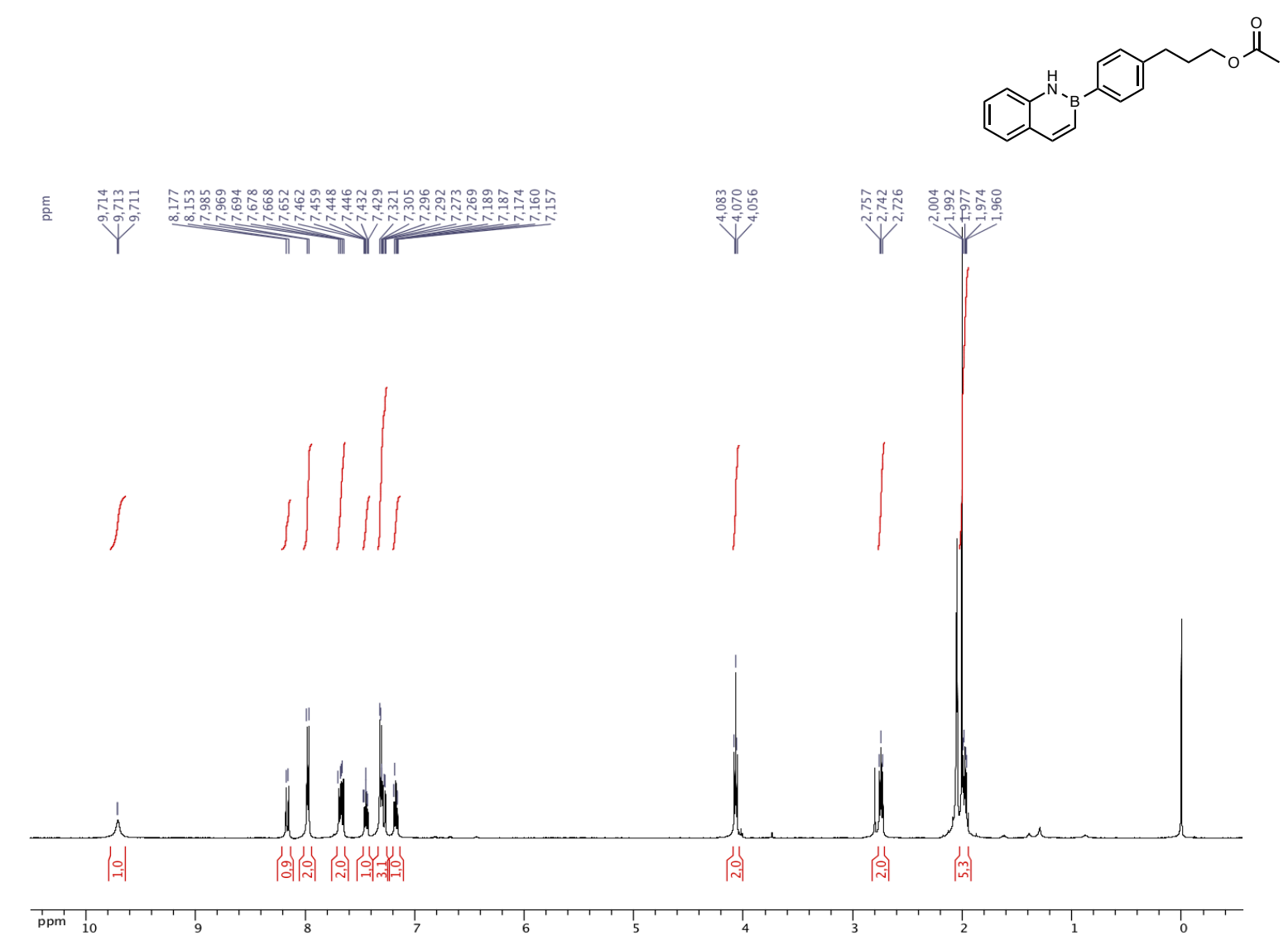

${ }^{1} \mathrm{H}$ NMR (acetone- $d_{6}, 500.4 \mathrm{MHz}$ ) of 2-(4-(3-acetoxypropyl)phenyl)-2,1-borazanaphthalene (2g)

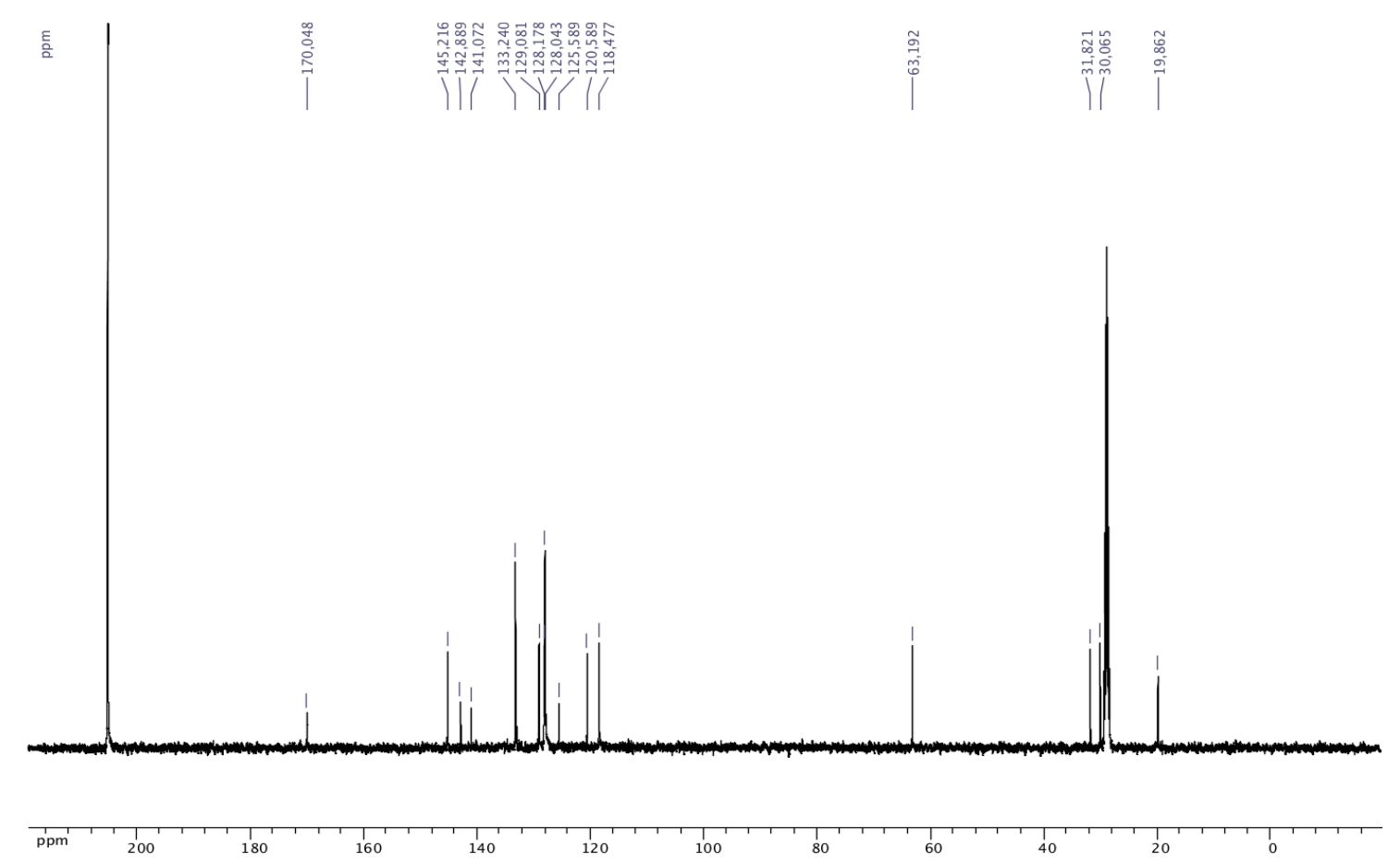

${ }^{13} \mathrm{C}\left\{{ }^{1} \mathrm{H}\right\}$ NMR (acetone- $d_{6}, 125.8 \mathrm{MHz}$ ) of 2-(4-(3-acetoxypropyl)phenyl)-2,1-borazanaphthalene (2g) 


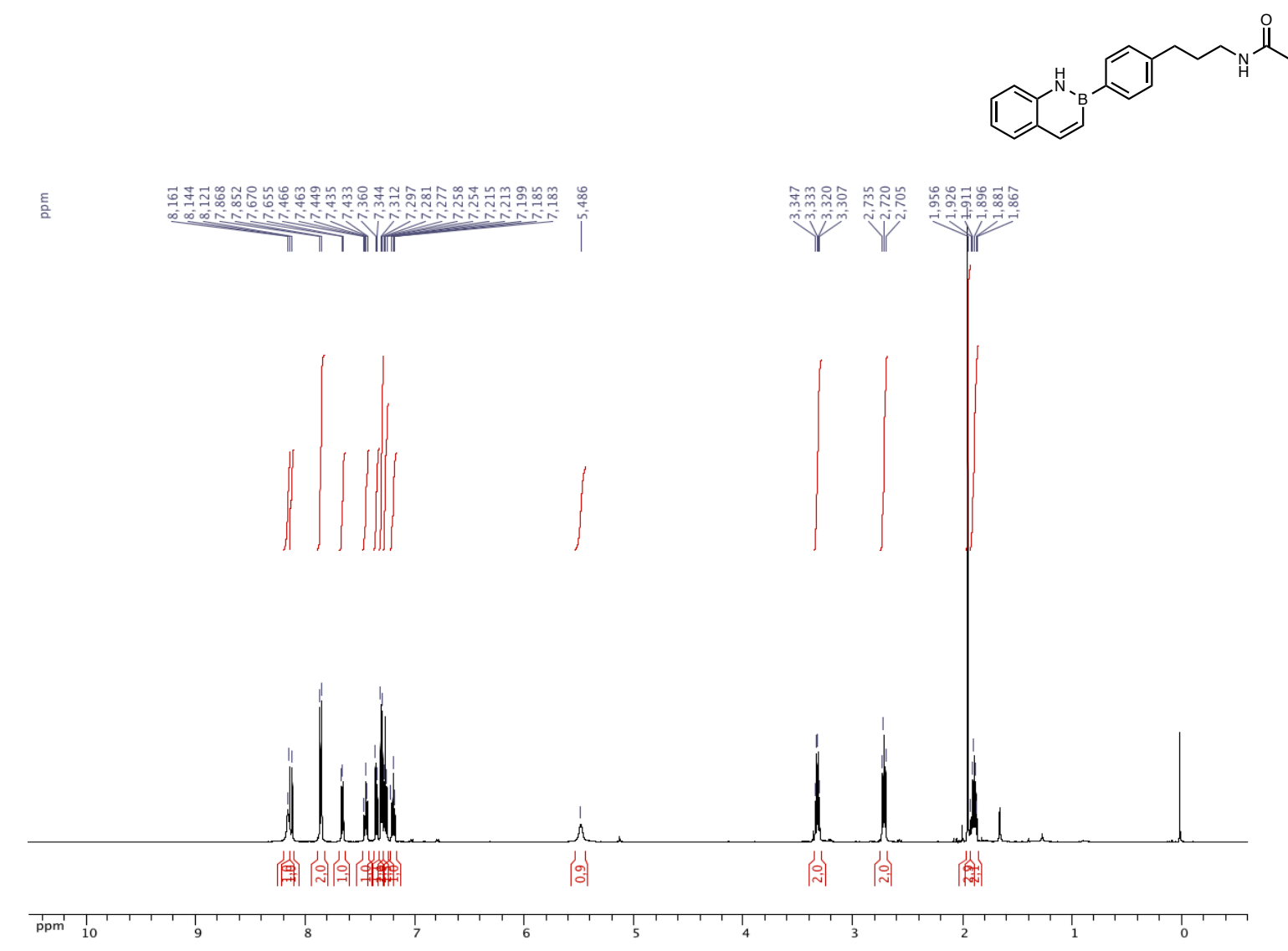

${ }^{1} \mathrm{H} \mathrm{NMR}\left(\mathrm{CDCl}_{3}, 500.4 \mathrm{MHz}\right)$ of 2-(4-(3-acetamidopropyl)phenyl)-2,1-borazanaphthalene (2h)
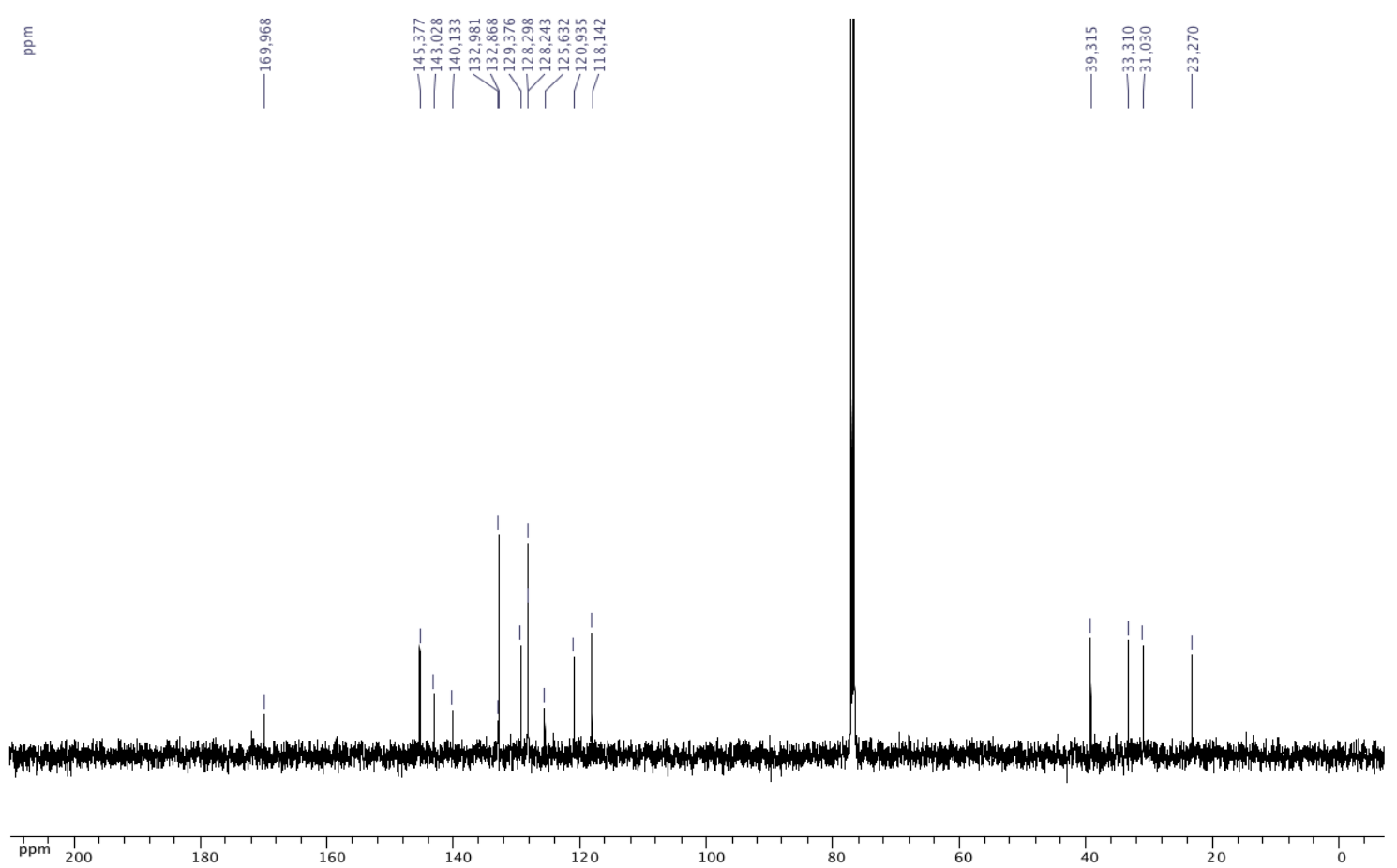

${ }^{13} \mathrm{C}\left\{{ }^{1} \mathrm{H}\right\}$ NMR $\left(\mathrm{CDCl}_{3}, 125.8 \mathrm{MHz}\right)$ of 2-(4-(3-acetamidopropyl)phenyl)-2,1-borazanaphthalene (2h) 


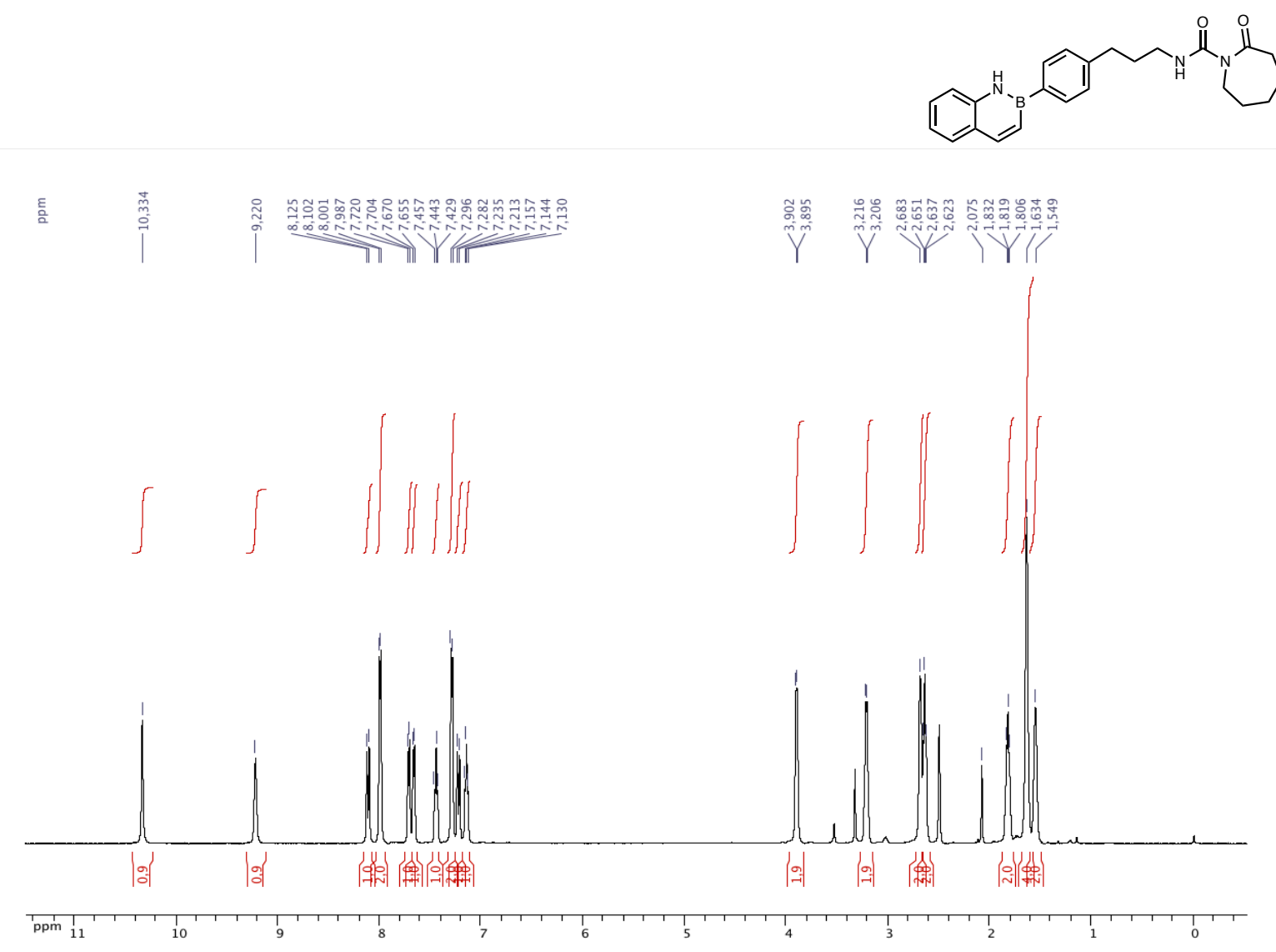

${ }^{1} \mathrm{H}$ NMR (DMSO- $d_{6}, 500.4 \mathrm{MHz}$ ) 2-(4-(3-(2-oxoazepane-1-carboxamido)propyl)phenyl)-2,1-borazanaphthalene (2i)
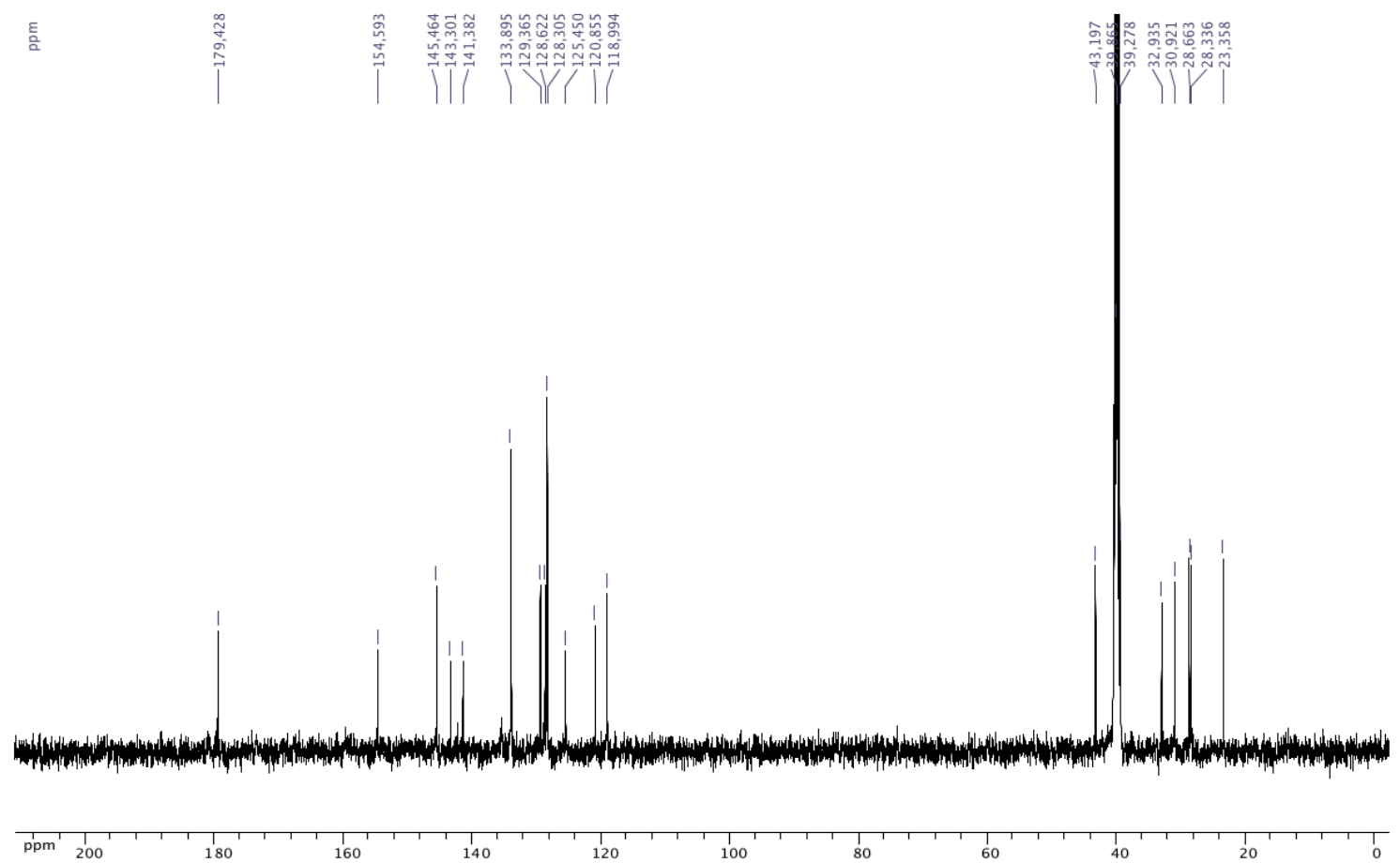

${ }^{13} \mathrm{C}\left\{{ }^{1} \mathrm{H}\right\}$ NMR (DMSO-d $\left.d_{6}, 125.8 \mathrm{MHz}\right)$ 2-(4-(3-(2-oxoazepane-1-carboxamido)propyl)phenyl)-2,1borazanaphthalene (2i) 


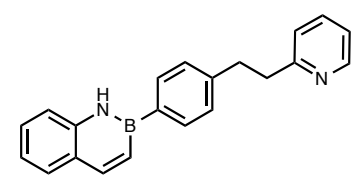

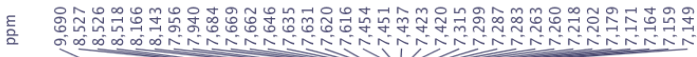

(1)
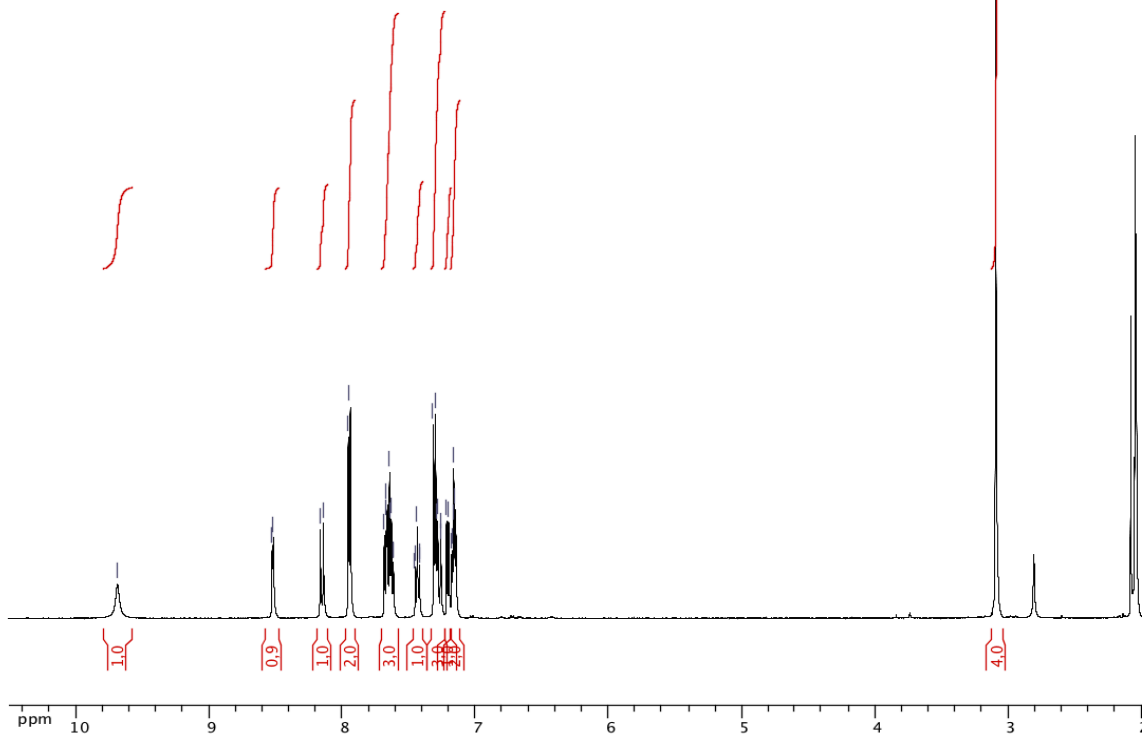

${ }^{1} \mathrm{H}$ NMR (acetone- $d_{6}, 500.4 \mathrm{MHz}$ ) of 2-(4-(2-pyridin-2-yl)ethyl)phenyl)-2,1-borazanaphthalene (2j)
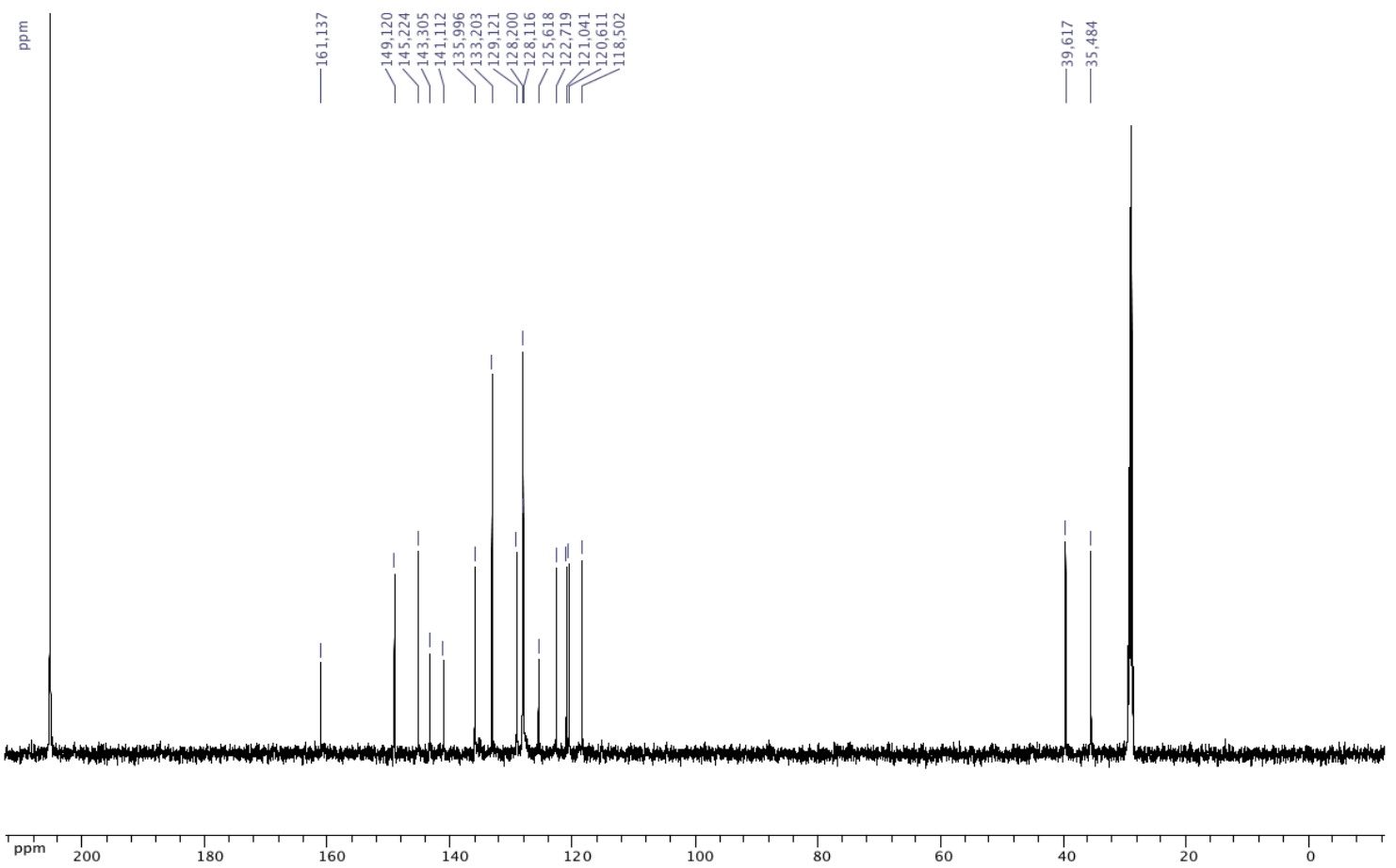

${ }^{13} \mathrm{C}\left\{{ }^{1} \mathrm{H}\right\}$ NMR (acetone- $d_{6}, 125.8 \mathrm{MHz}$ ) of 2-(4-(2-pyridin-2-yl)ethyl)phenyl)-2,1-borazanaphthalene (2j) 


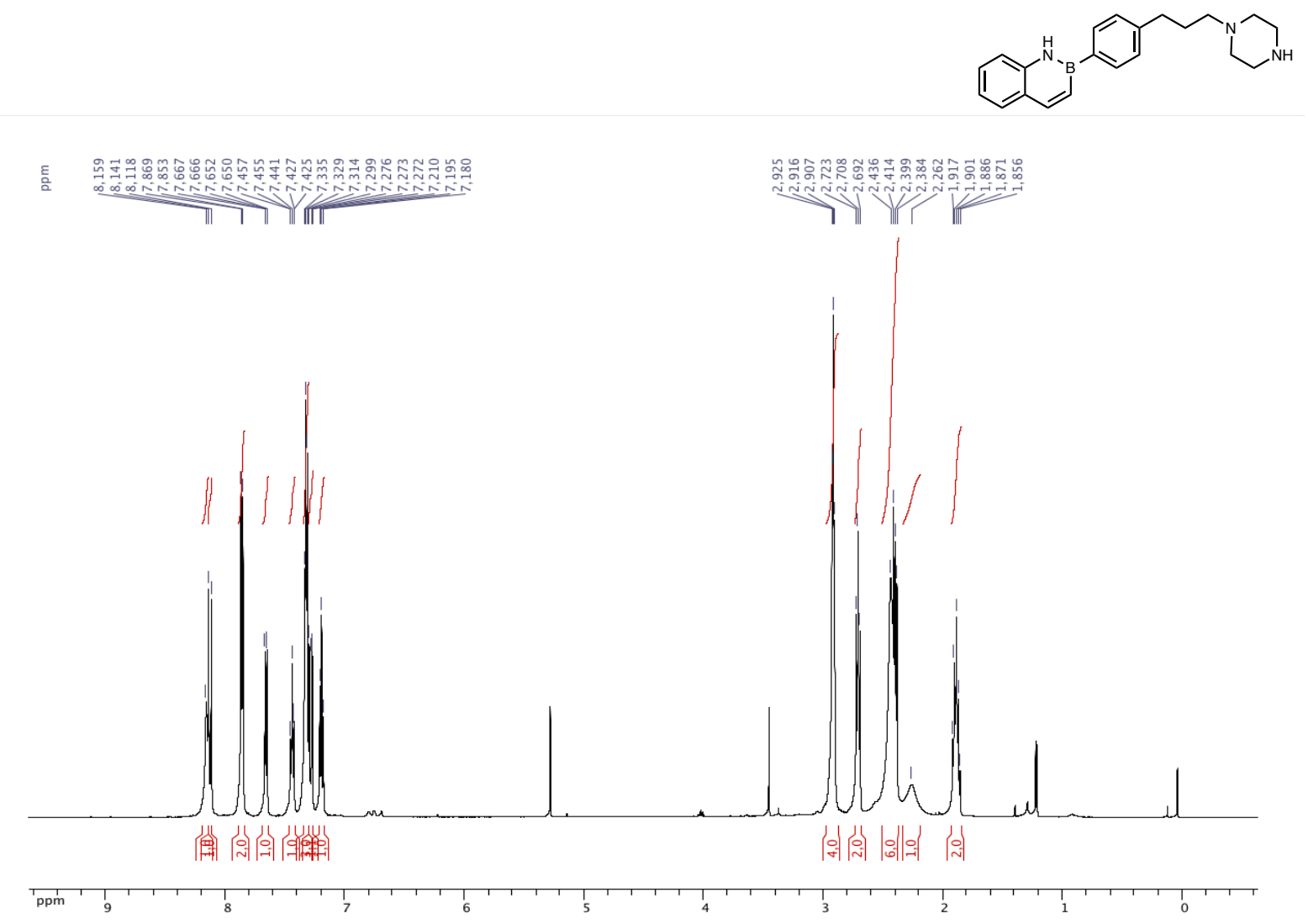

${ }^{1}$ H NMR (acetone- $d_{6}, 500.4$ MHz) of 2-(4-(3-(piperazin-1-yl)propyl)phenyl)-2,1-borazanaphthalene (2k)
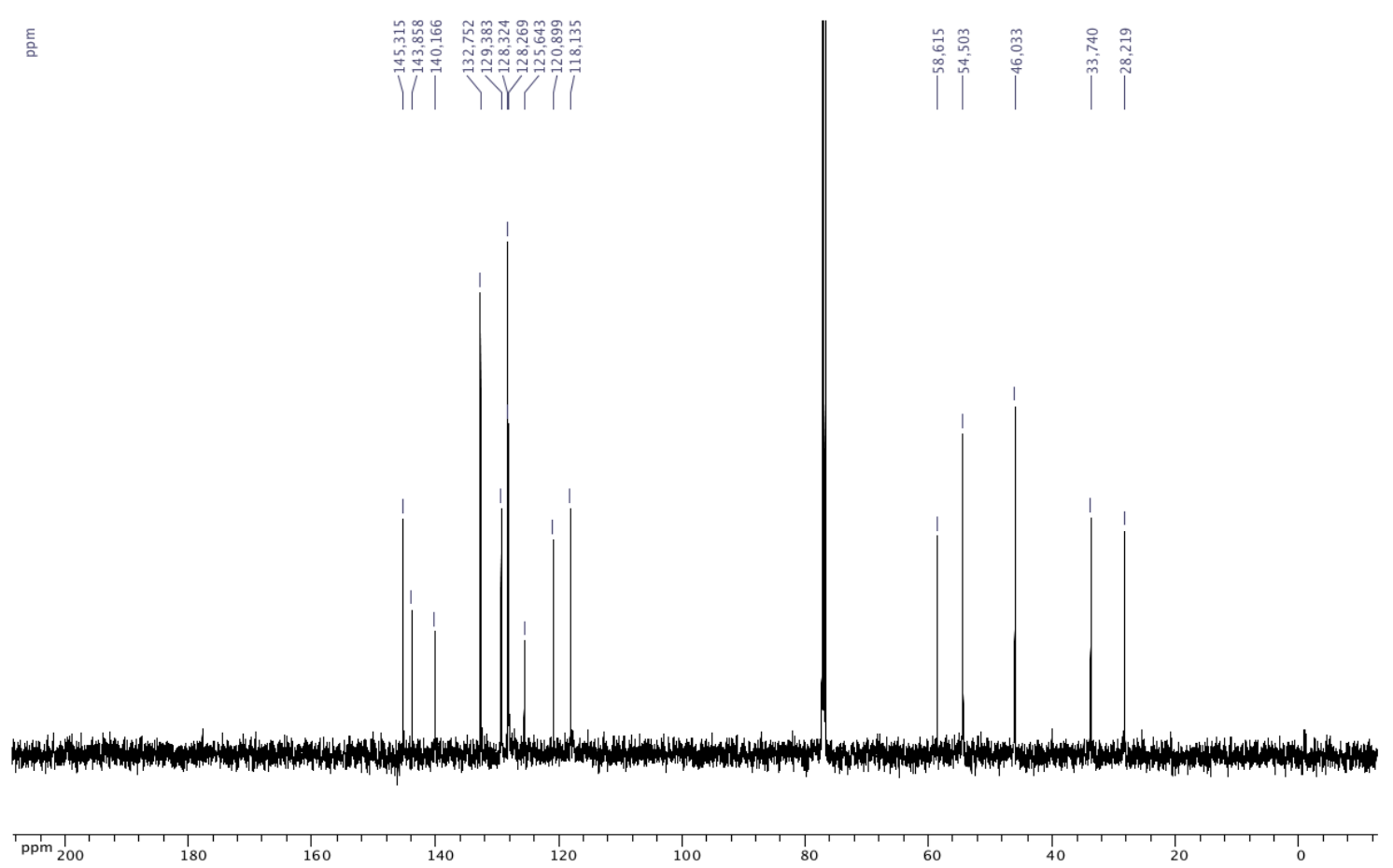

${ }^{13} \mathrm{C}\left\{{ }^{1} \mathrm{H}\right\}$ NMR (acetone- $\left.d_{6}, 125.8 \mathrm{MHz}\right)$ of 2-(4-(3-(piperazin-1-yl)propyl)phenyl)-2,1-borazanaphthalene (2k) 


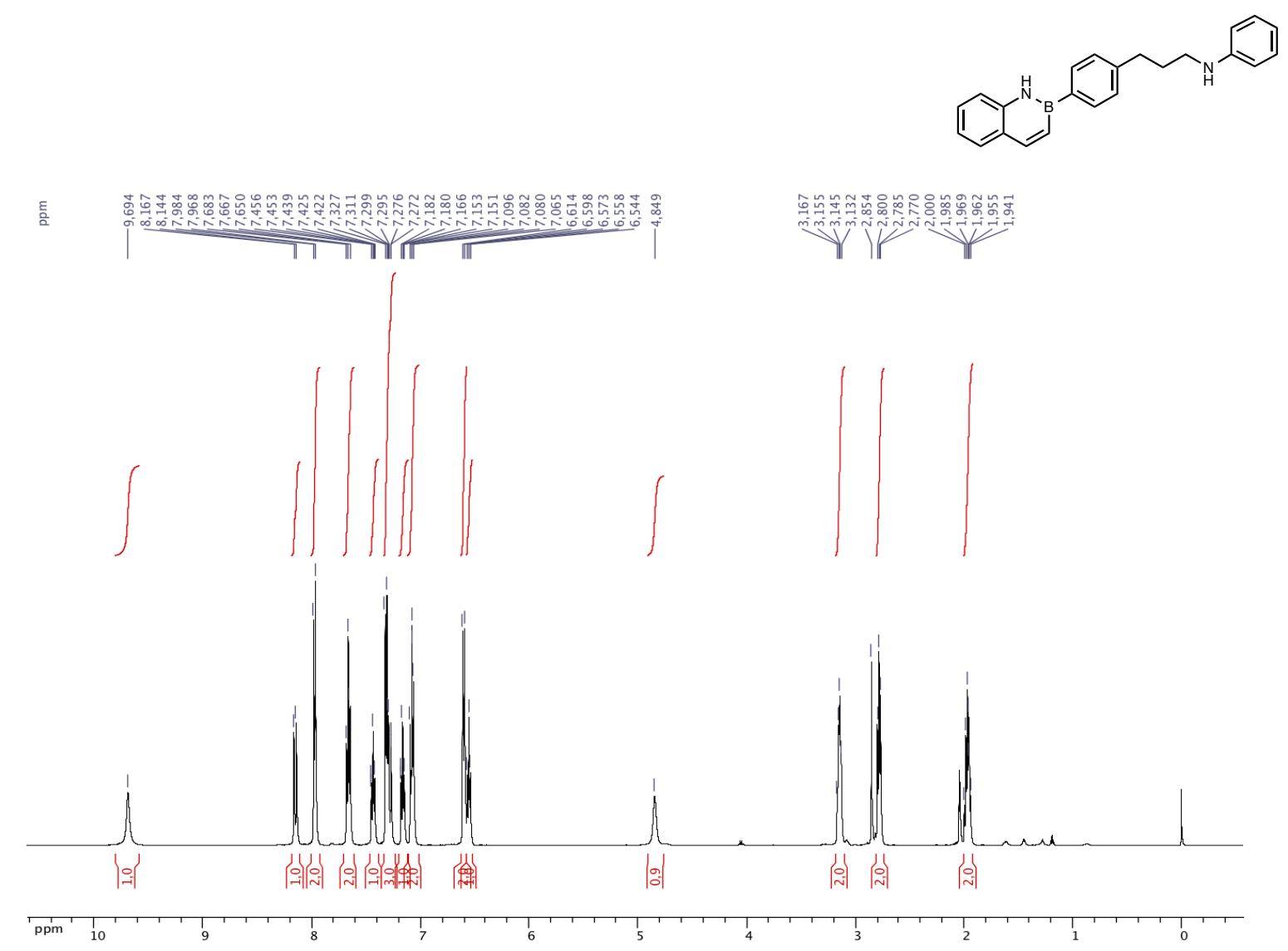

${ }^{1} \mathrm{H}$ NMR (acetone- $d_{6}, 500.4 \mathrm{MHz}$ ) of 2-(4-(3-(phenylamino)propyl)phenyl)-2,1-borazanaphthalene (2I)

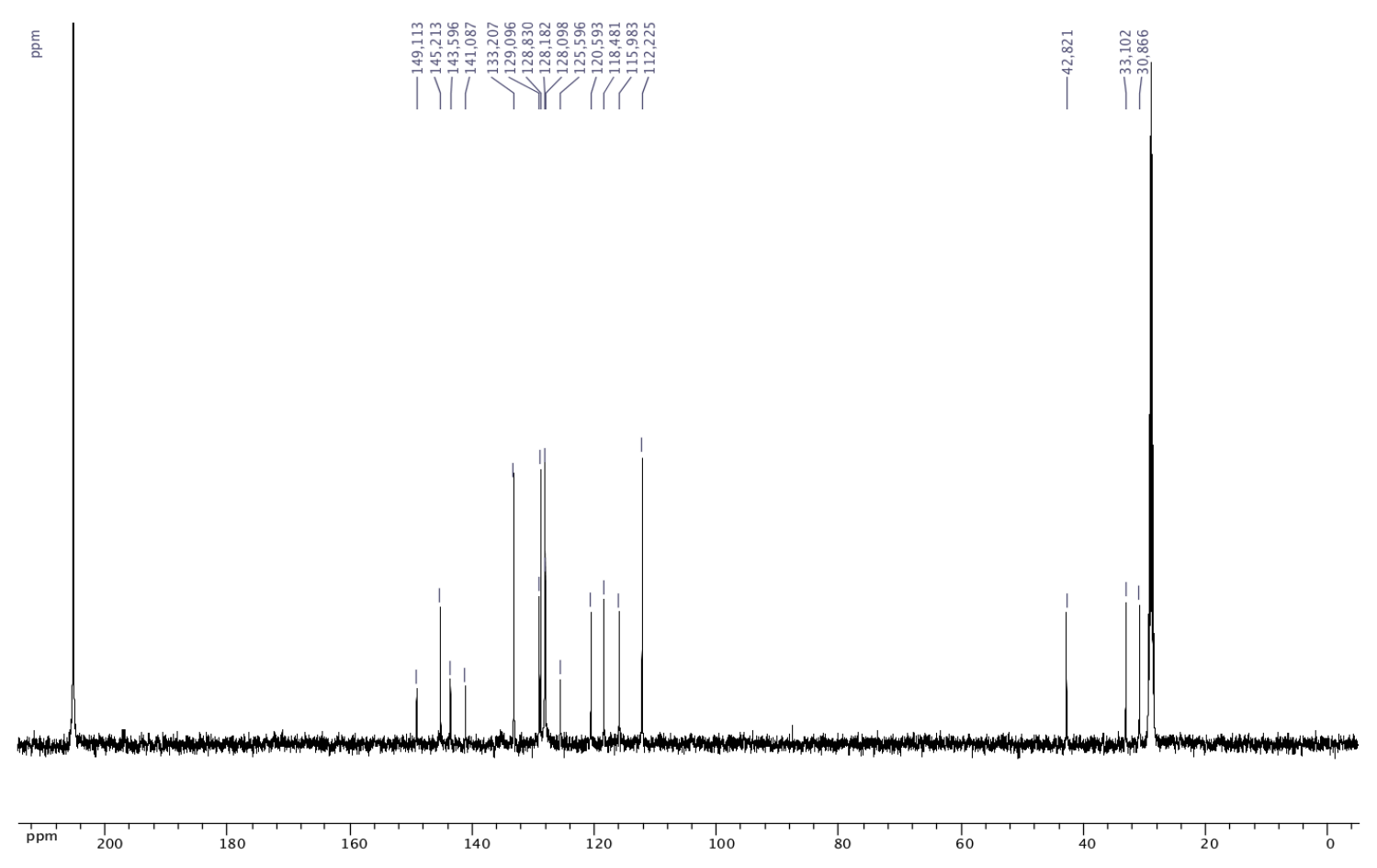

${ }^{13} \mathrm{C}\left\{{ }^{1} \mathrm{H}\right\}$ NMR (acetone- $d_{6}, 125.8 \mathrm{MHz}$ ) of 2-(4-(3-(phenylamino)propyl)phenyl)-2,1-borazanaphthalene (2l) 


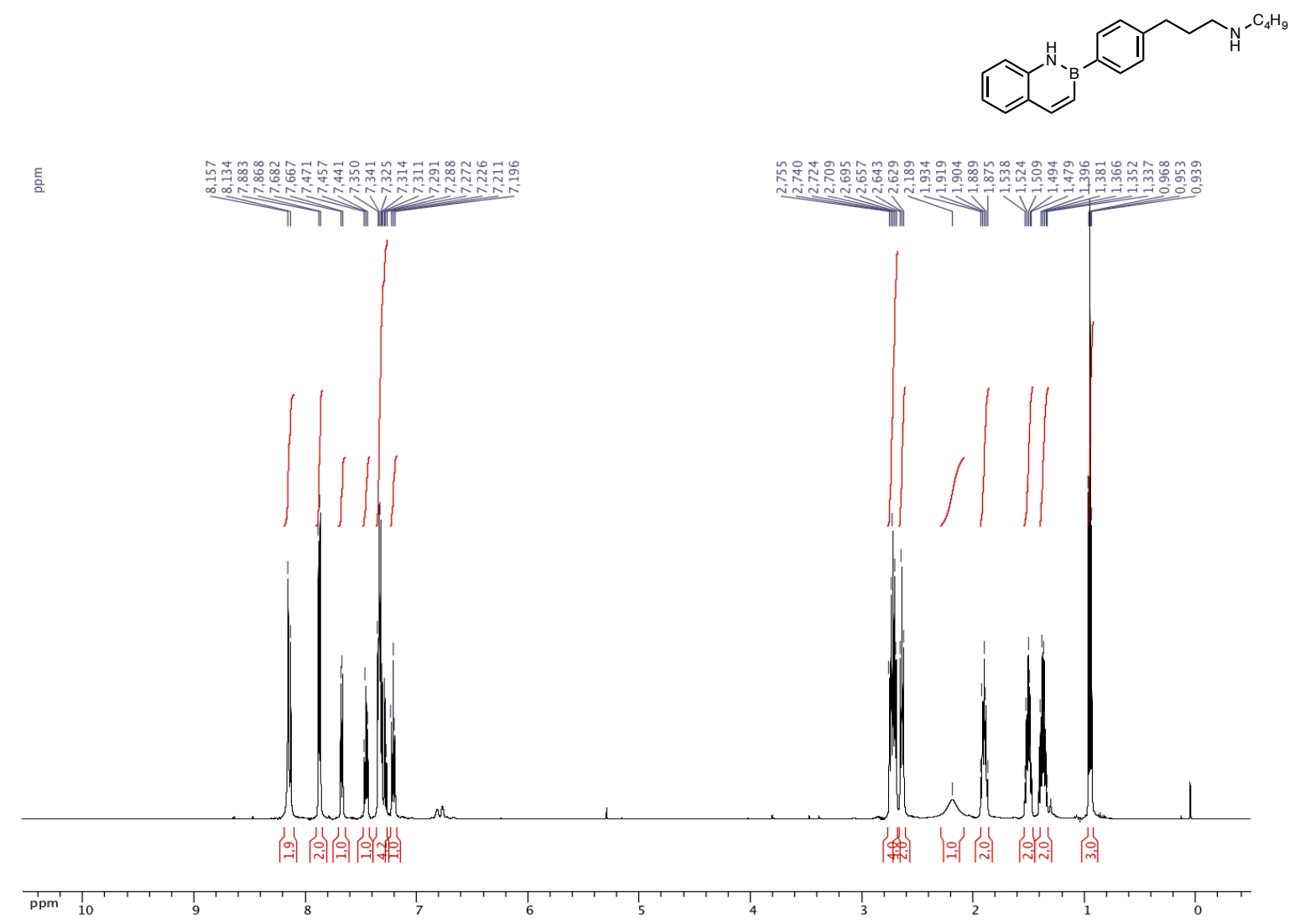

${ }^{1} \mathrm{H}$ NMR ( $\left.\mathrm{CDCl}_{3}, 500.4 \mathrm{MHz}\right)$ of 2-(4-(3-(butylamino)propyl)phenyl)-2,1-borazanaphthalene (2m)

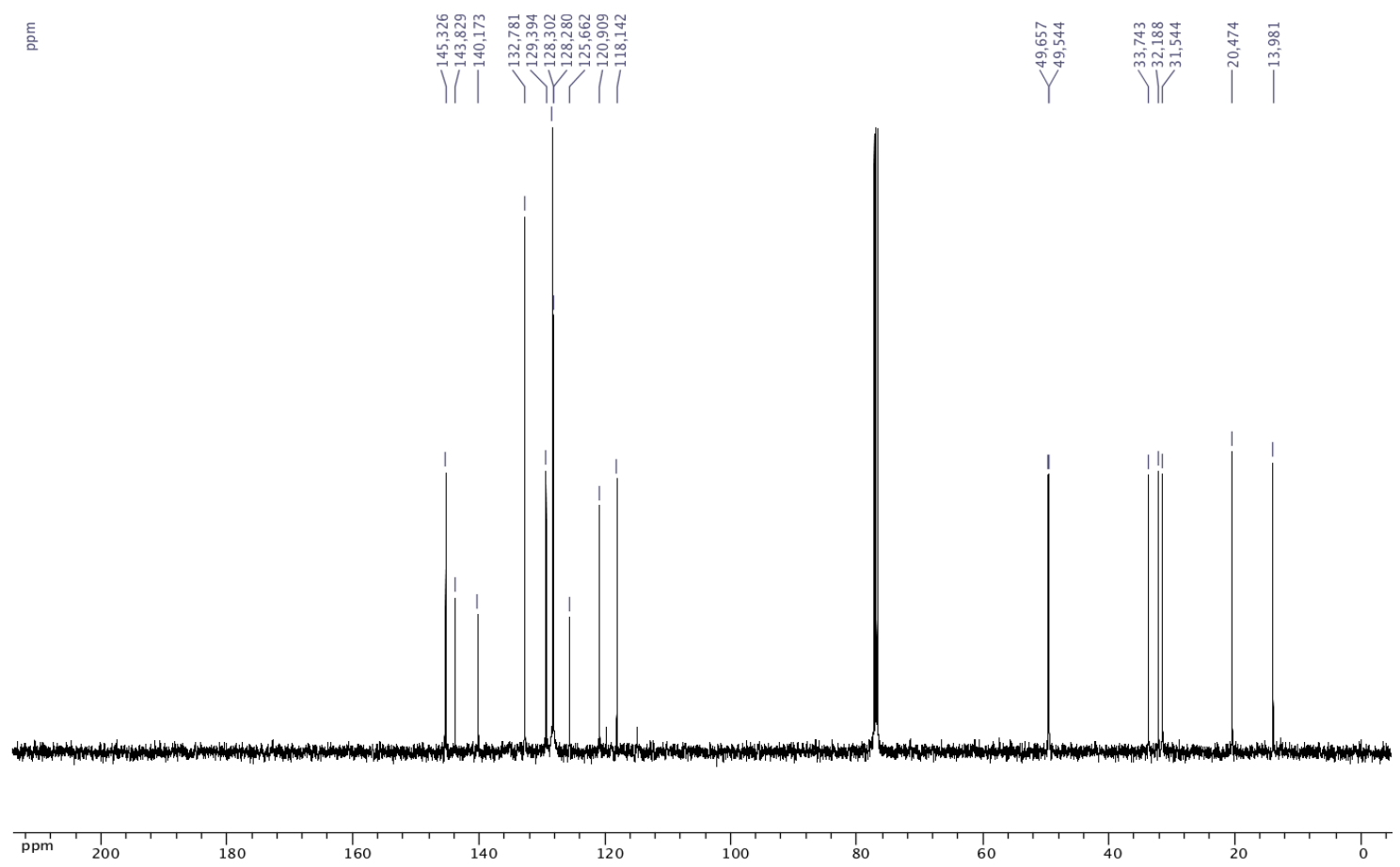

${ }^{13} \mathrm{C}\left\{{ }^{1} \mathrm{H}\right\} \mathrm{NMR}\left(\mathrm{CDCl}_{3}, 125.8 \mathrm{MHz}\right)$ of 2-(4-(3-(butylamino)propyl)phenyl)-2,1-borazanaphthalene (2m) 


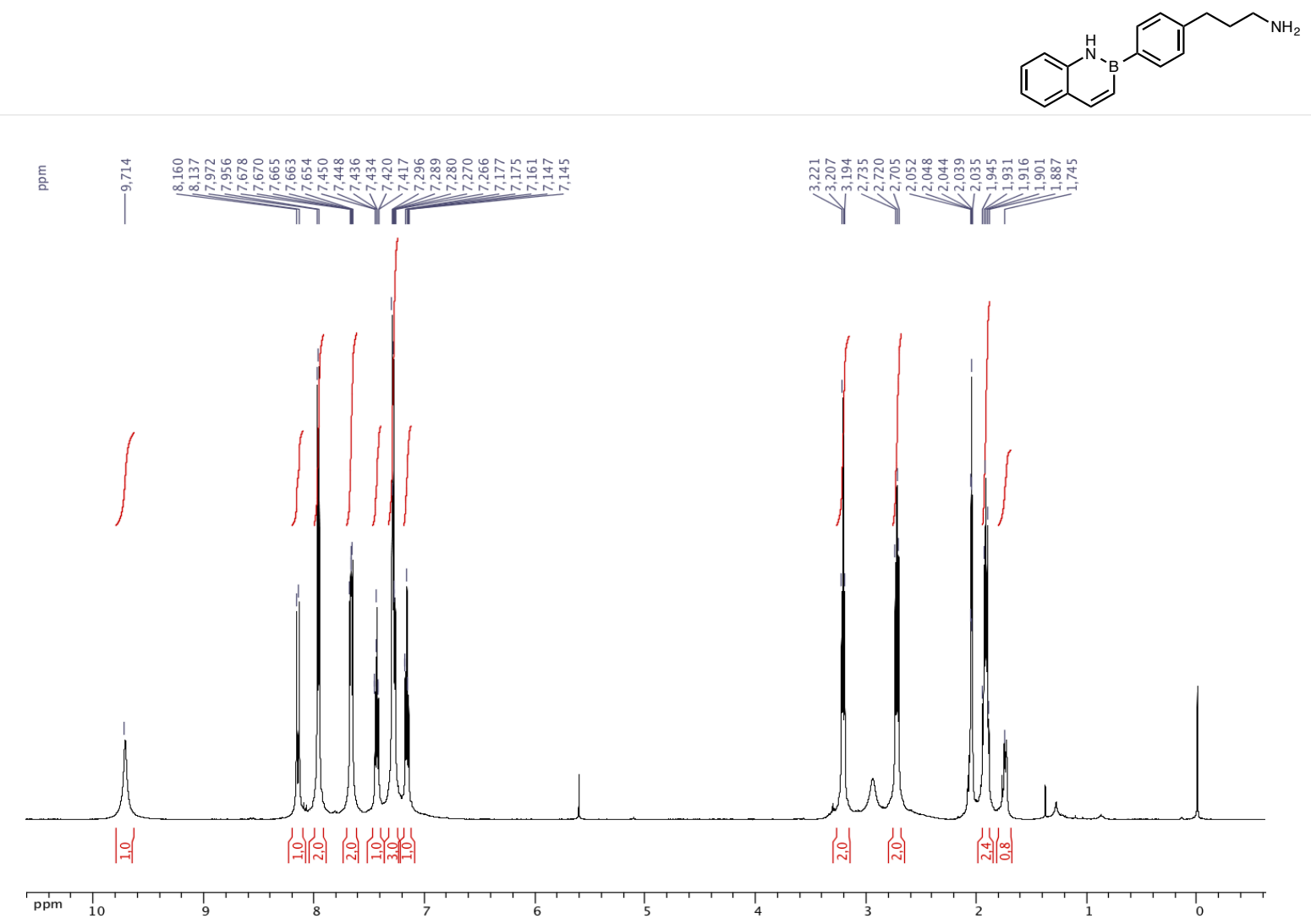

${ }^{1} \mathrm{H}$ NMR (acetone- $\left.d_{6}, 500.4 \mathrm{MHz}\right)$ of 2-(4-(3-aminopropyl)phenyl)-2,1-borazanaphthalene (2n)
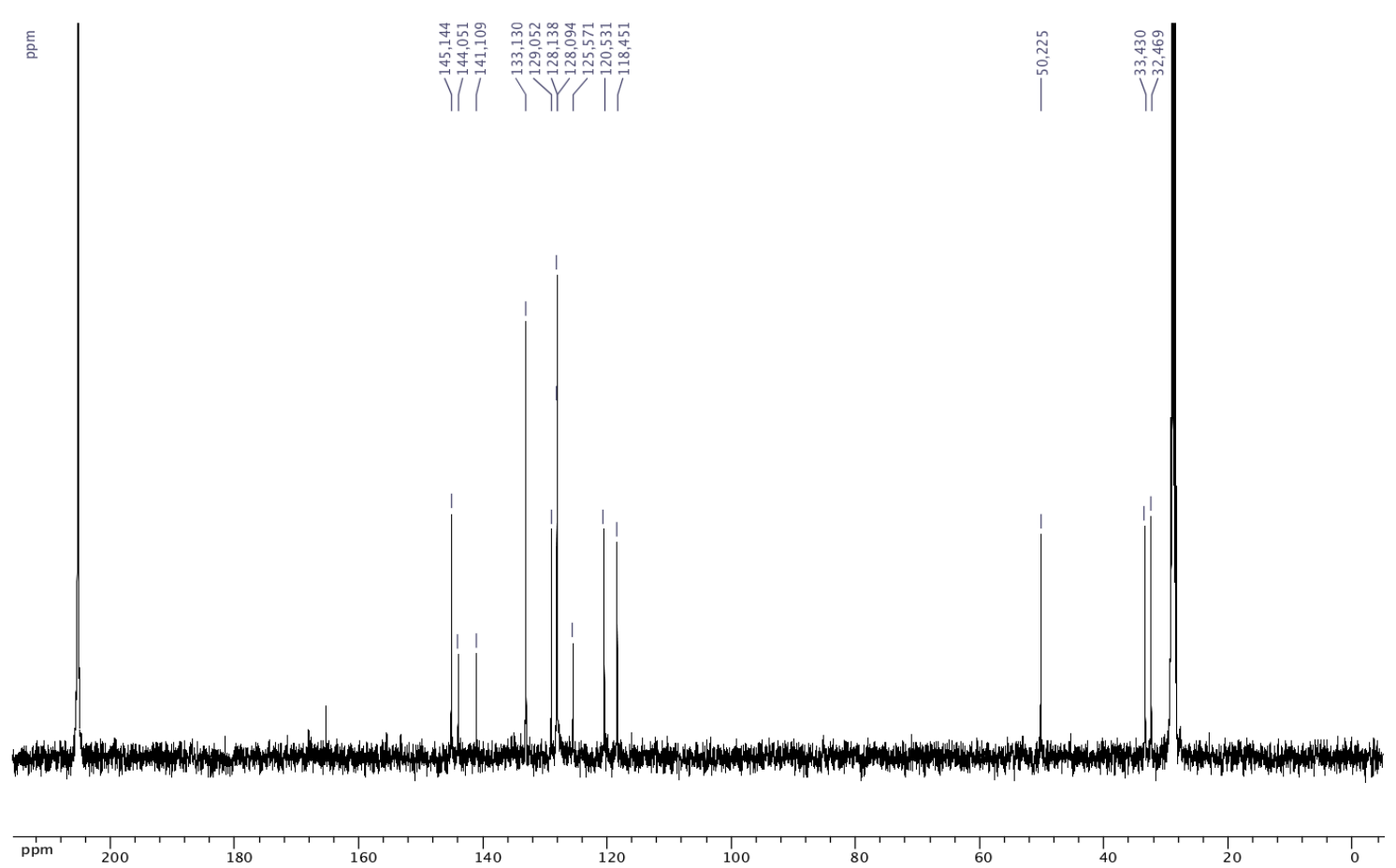

${ }^{13} \mathrm{C}\left\{{ }^{1} \mathrm{H}\right\}$ NMR (acetone- $\left.d_{6}, 125.8 \mathrm{MHz}\right)$ of 2-(4-(3-aminopropyl)phenyl)-2,1-borazanaphthalene (2n) 


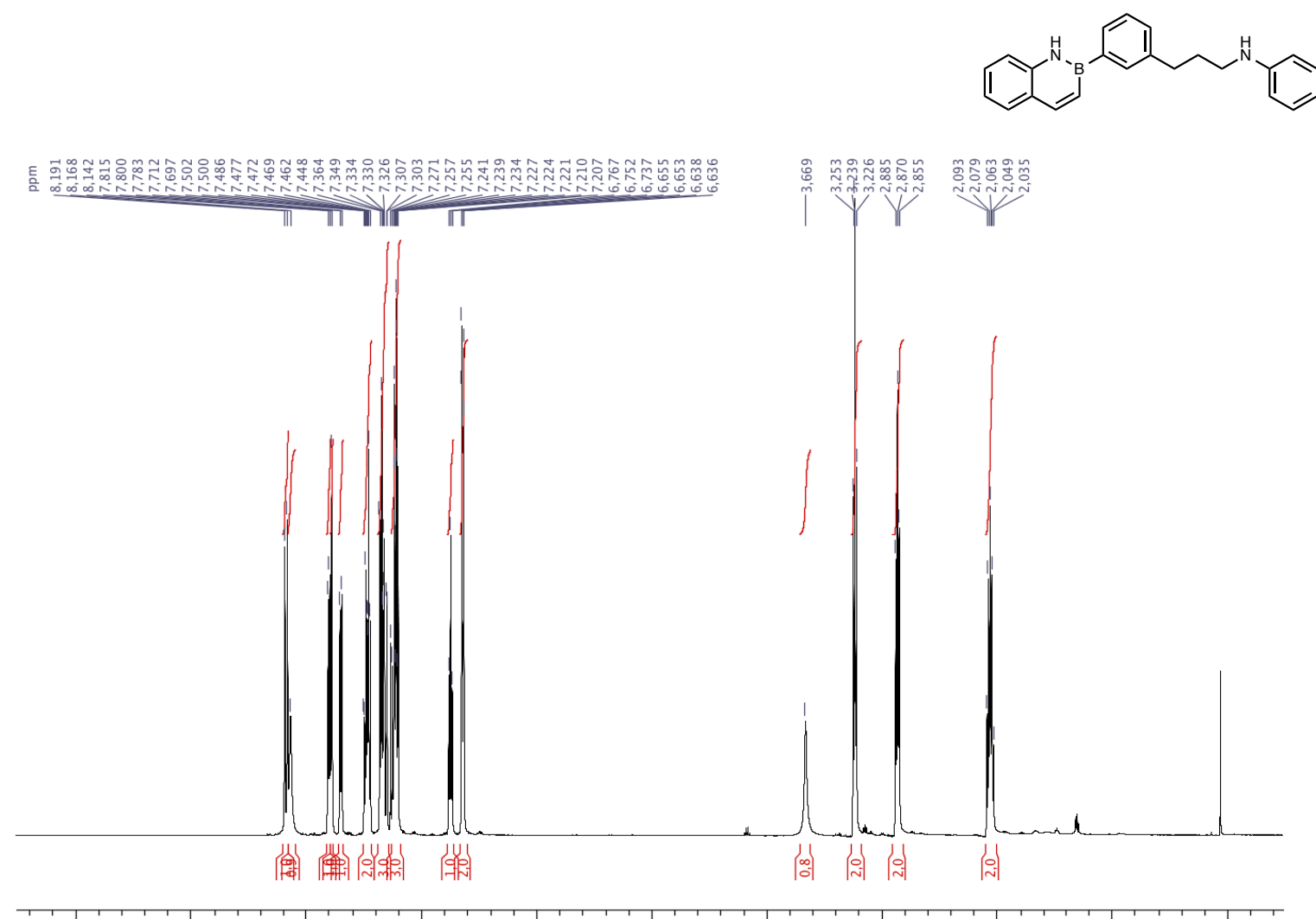

${ }^{1} \mathrm{H} \mathrm{NMR}\left(\mathrm{CDCl}_{3}, 500.4 \mathrm{MHz}\right)$ of 2-(3-(3-(phenylamino)propyl)phenyl)-2,1-borazanaphthalene (3a)

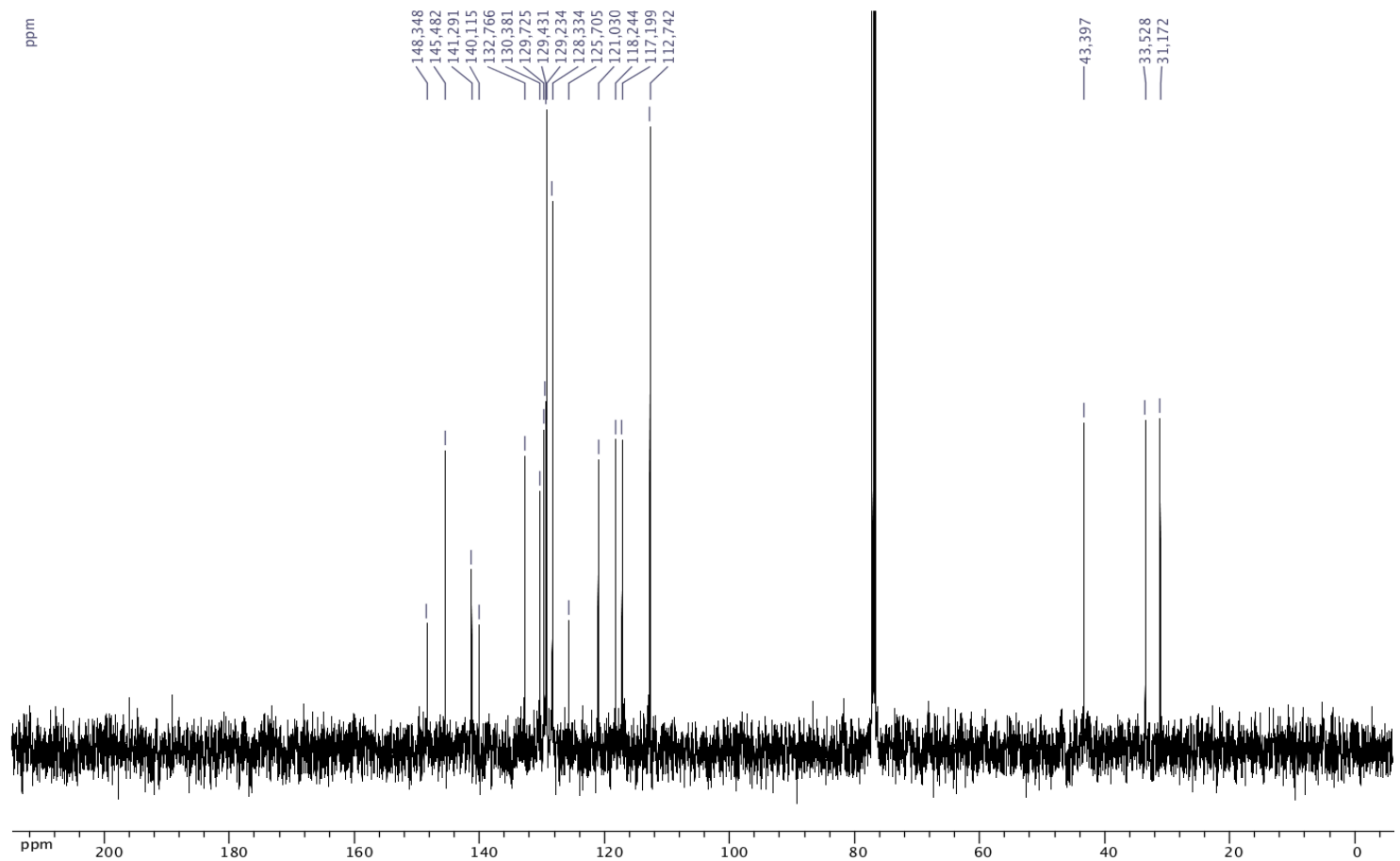

${ }^{13} \mathrm{C}\left\{{ }^{1} \mathrm{H}\right\}$ NMR $\left(\mathrm{CDCl}_{3}, 125.8 \mathrm{MHz}\right)$ of 2-(3-(3-(phenylamino)propyl)phenyl)-2,1-borazanaphthalene (3a) 


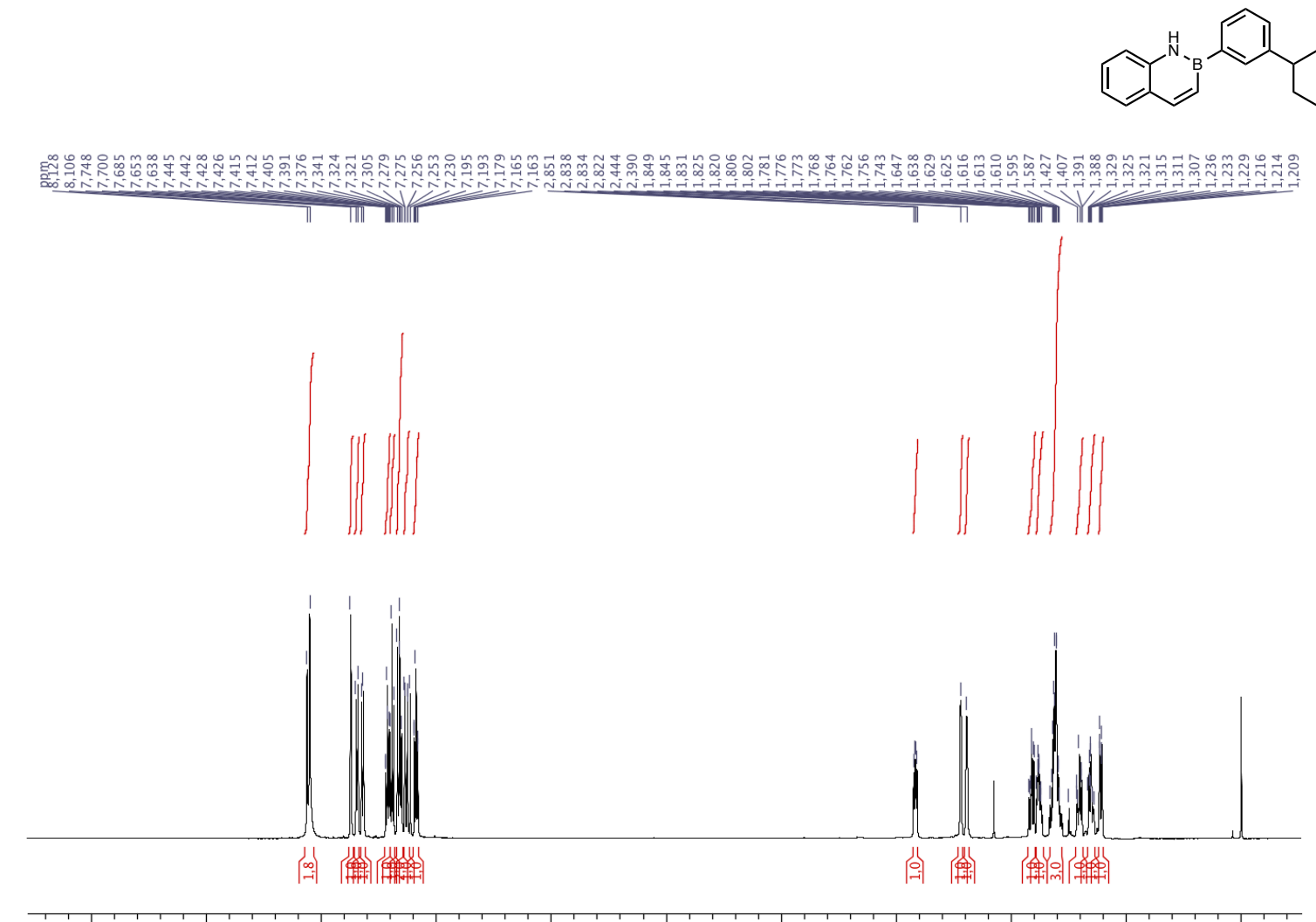

${ }^{1} \mathrm{H} \mathrm{NMR}\left(\mathrm{CDCl}_{3}, 500.4 \mathrm{MHz}\right)$ of 2-(3-(exo-bicyclo[2.2.1]heptan-2-yl)phenyl)-2,1-borazanaphthalene (3b)

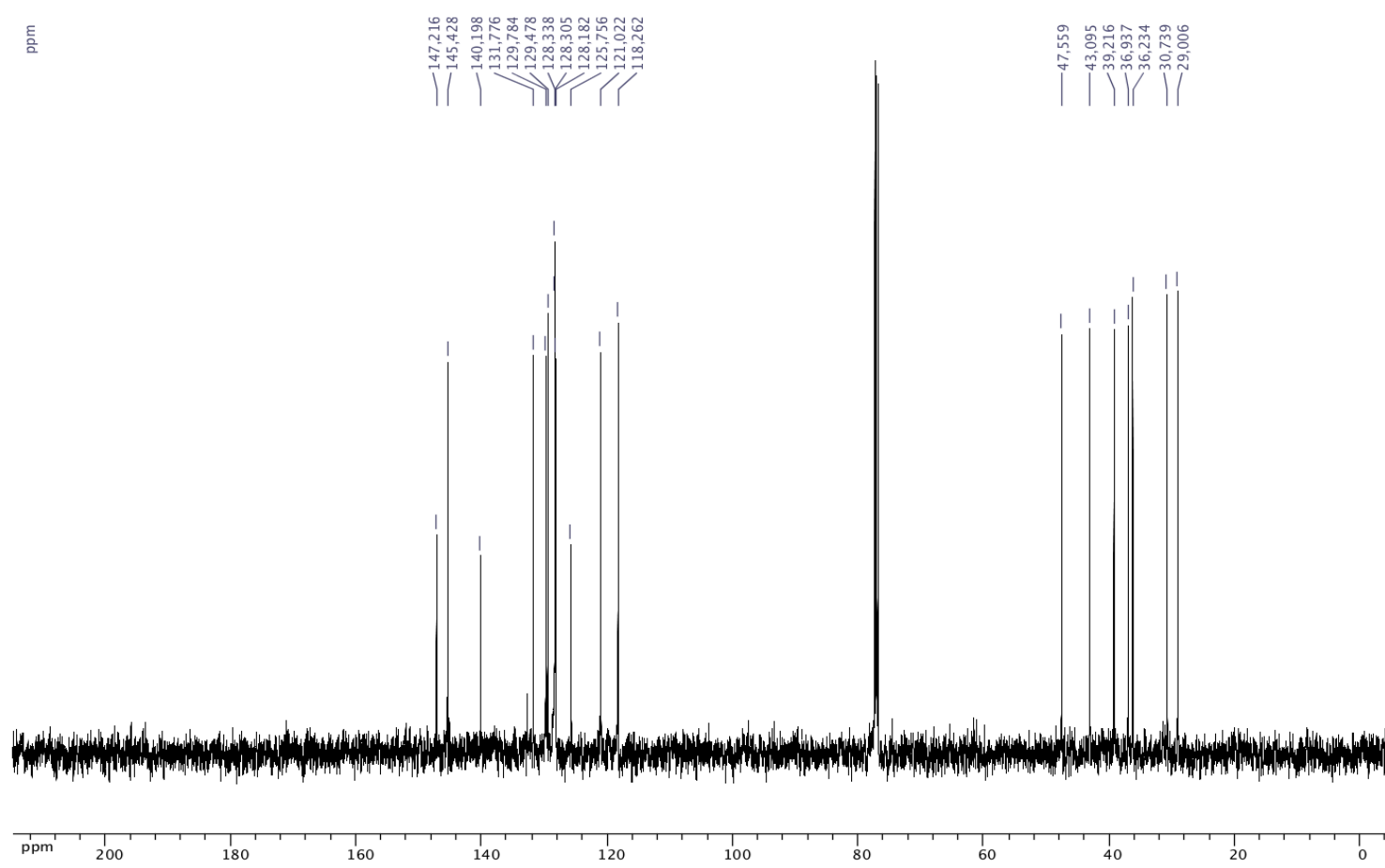

${ }^{13} \mathrm{C}\left\{{ }^{1} \mathrm{H}\right\}$ NMR $\left(\mathrm{CDCl}_{3}, 125.8 \mathrm{MHz}\right)$ of 2-(3-(exo-bicyclo[2.2.1]heptan-2-yl)phenyl)-2,1-borazanaphthalene (3b) 


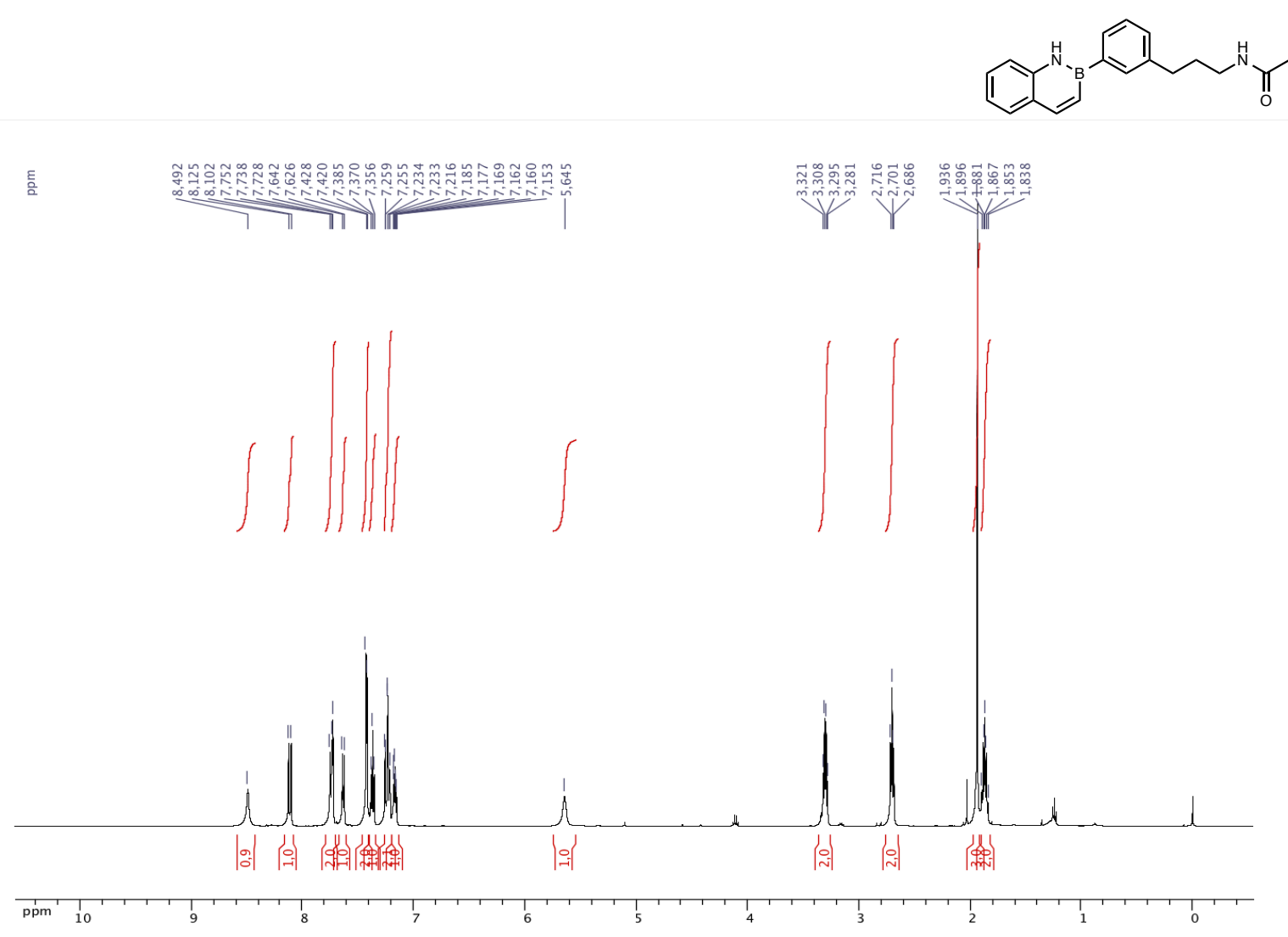

${ }^{1} \mathrm{H}$ NMR (CDCl $\left.3,500.4 \mathrm{MHz}\right)$ of 2-(3-(3-acetamidopropyl)phenyl)-2,1-borazanaphthalene (3c)
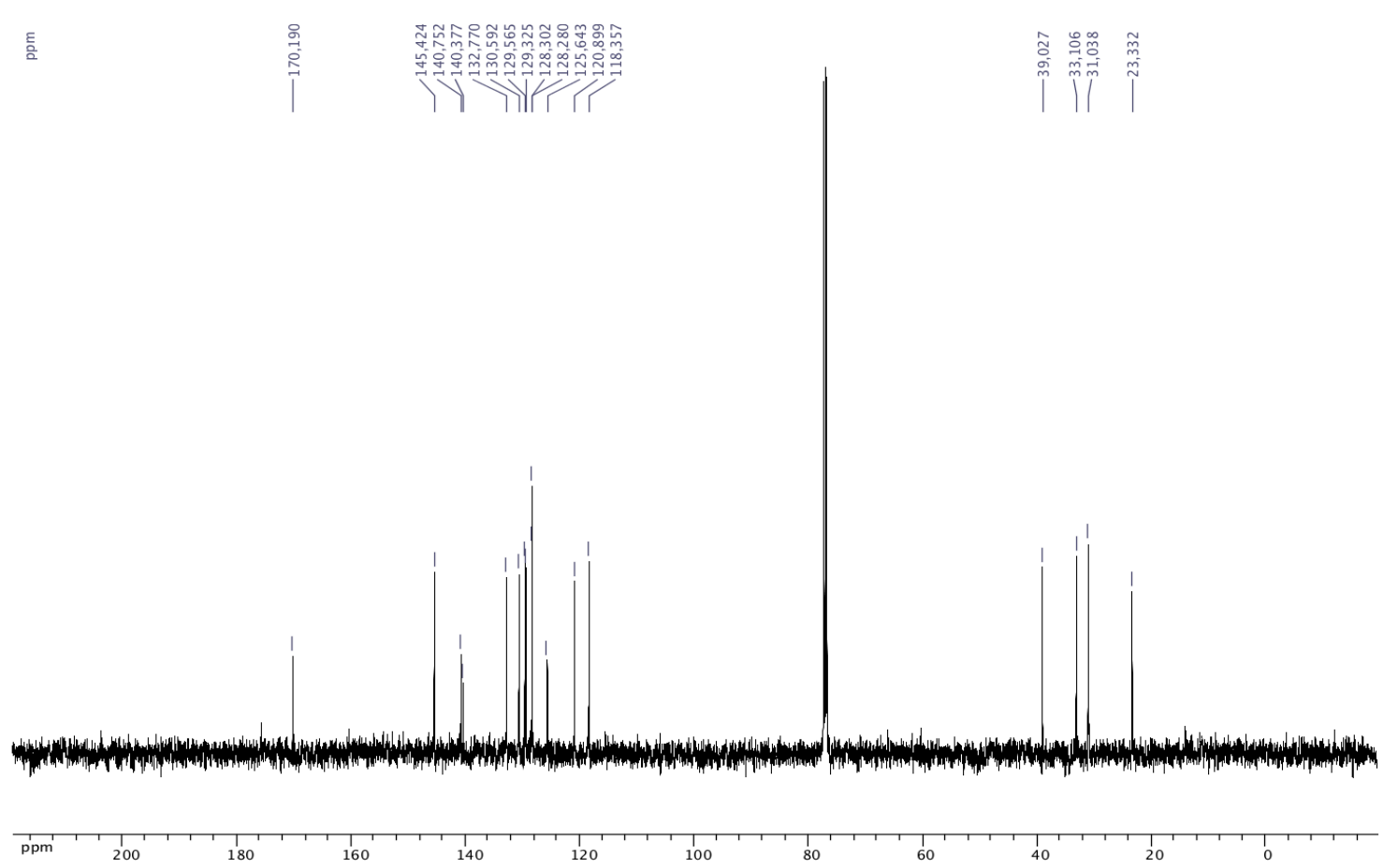

${ }^{13} \mathrm{C}\left\{{ }^{1} \mathrm{H}\right\} \mathrm{NMR}\left(\mathrm{CDCl}_{3}, 125.8 \mathrm{MHz}\right)$ of 2-(3-(3-acetamidopropyl)phenyl)-2,1-borazanaphthalene (3c) 


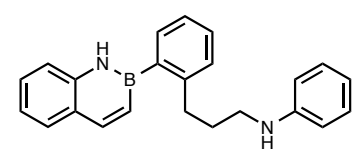

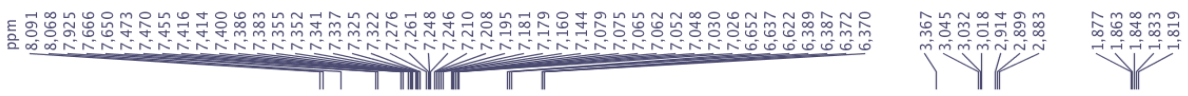

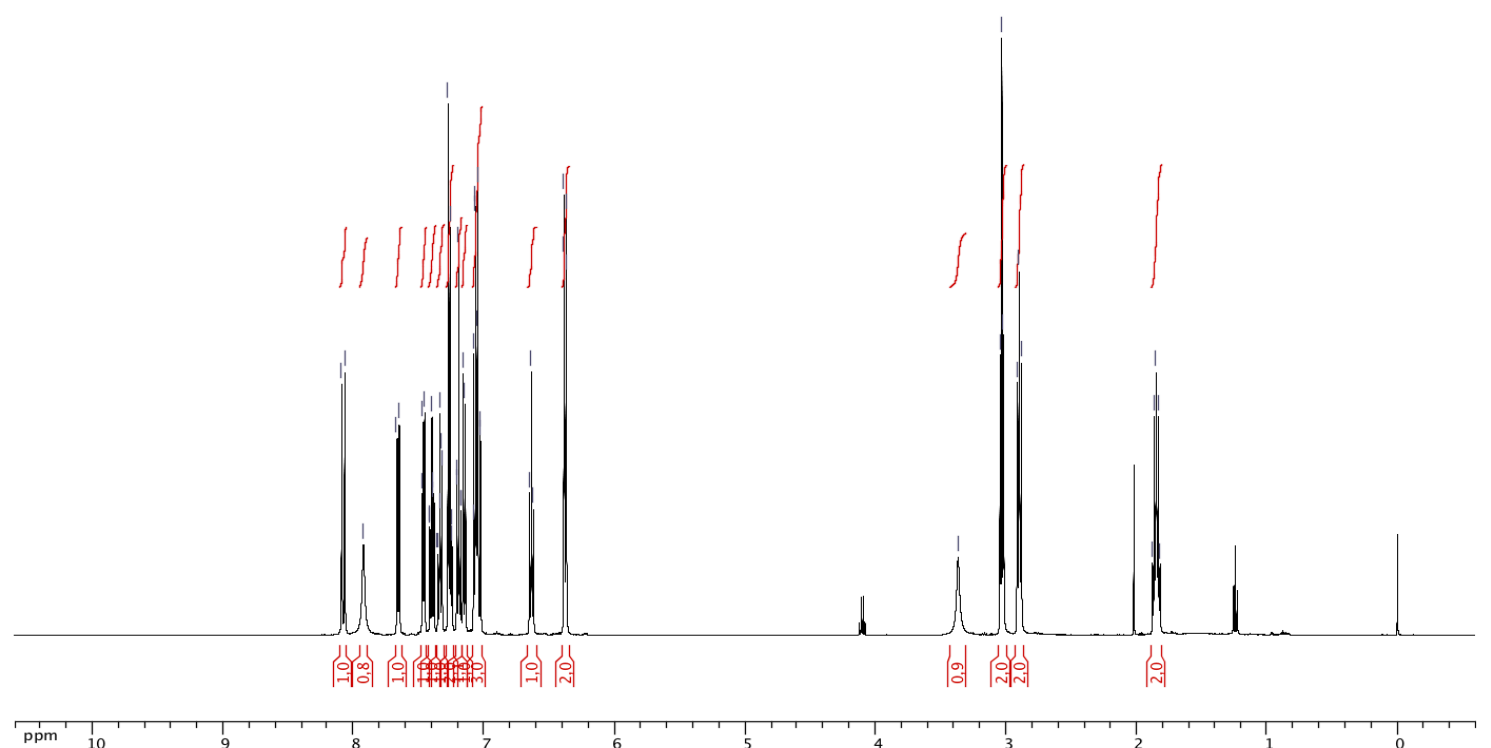

${ }^{1} \mathrm{H} \mathrm{NMR}\left(\mathrm{CDCl}_{3}, 500.4 \mathrm{MHz}\right)$ of 2-(2-(3-(phenylamino)propyl)phenyl)-2,1-borazanaphthalene (4a) 言

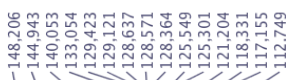
1

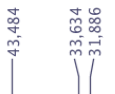

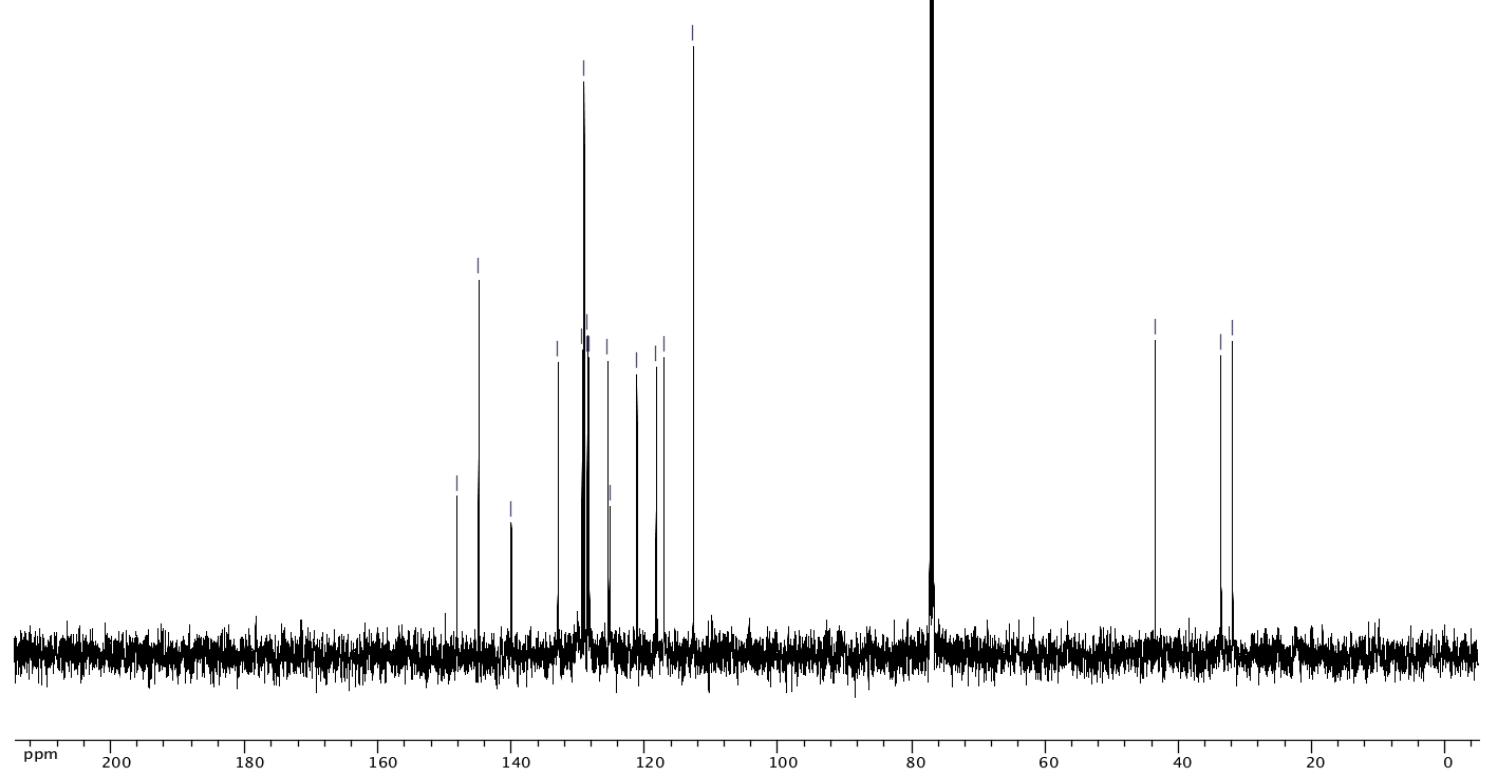

${ }^{13} \mathrm{C}\left\{{ }^{1} \mathrm{H}\right\} \mathrm{NMR}\left(\mathrm{CDCl}_{3}, 125.8 \mathrm{MHz}\right)$ of 2-(2-(3-(phenylamino)propyl)phenyl)-2,1-borazanaphthalene (4a) 


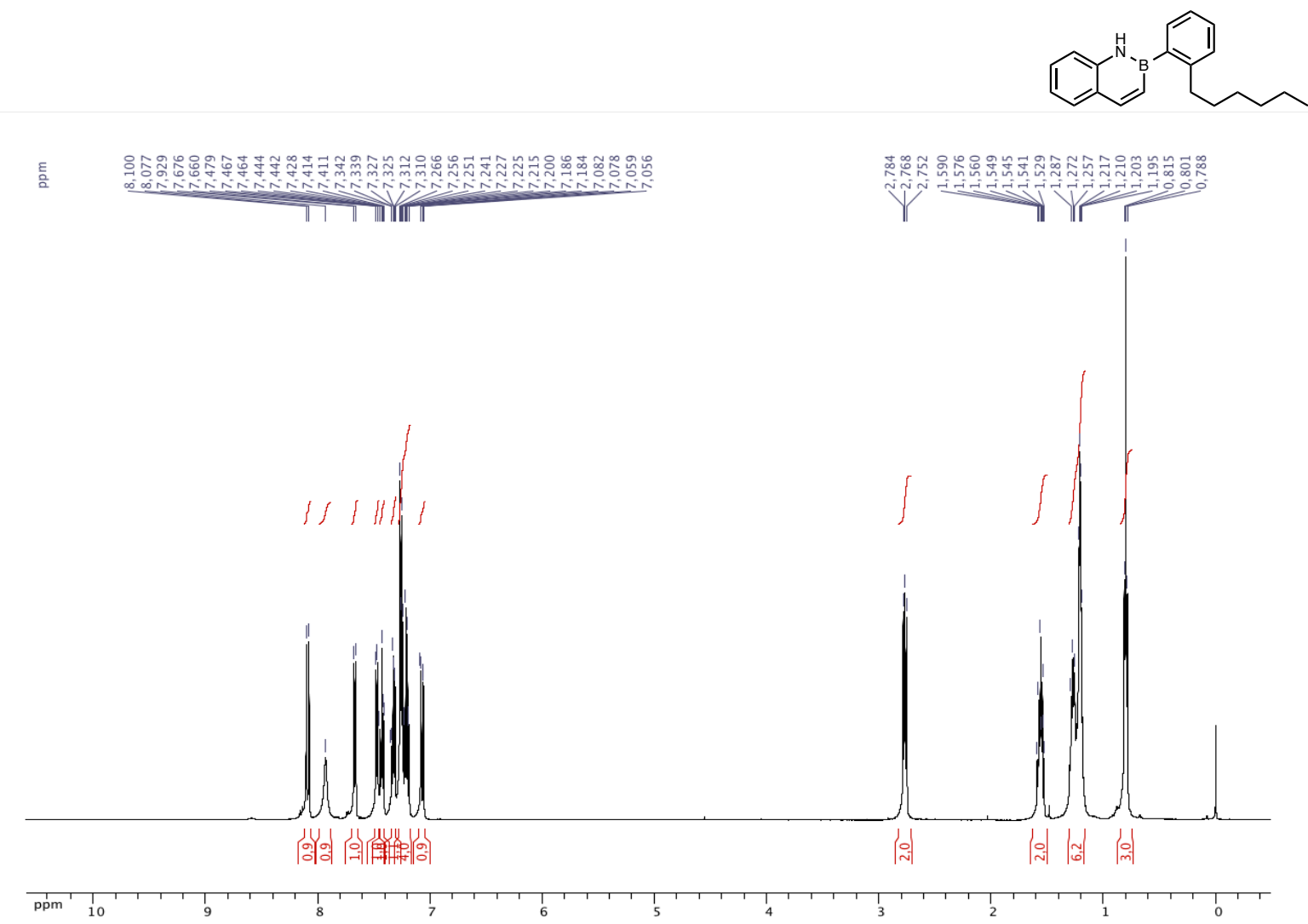

${ }^{1} \mathrm{H} \mathrm{NMR}\left(\mathrm{CDCl}_{3}, 500.4 \mathrm{MHz}\right)$ of 2-(2-hexylphenyl)-2,1-borazanaphthalene (4b)

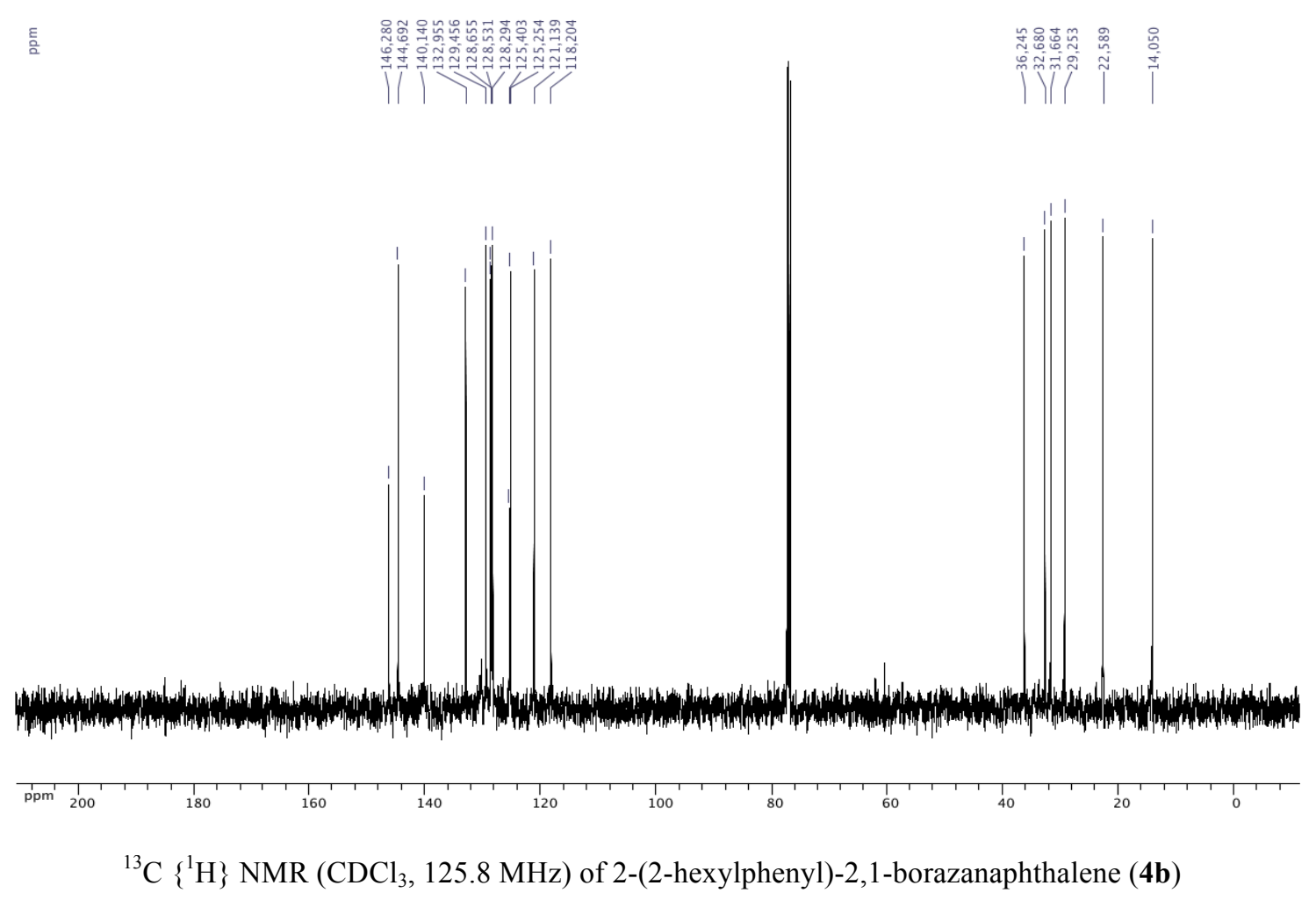




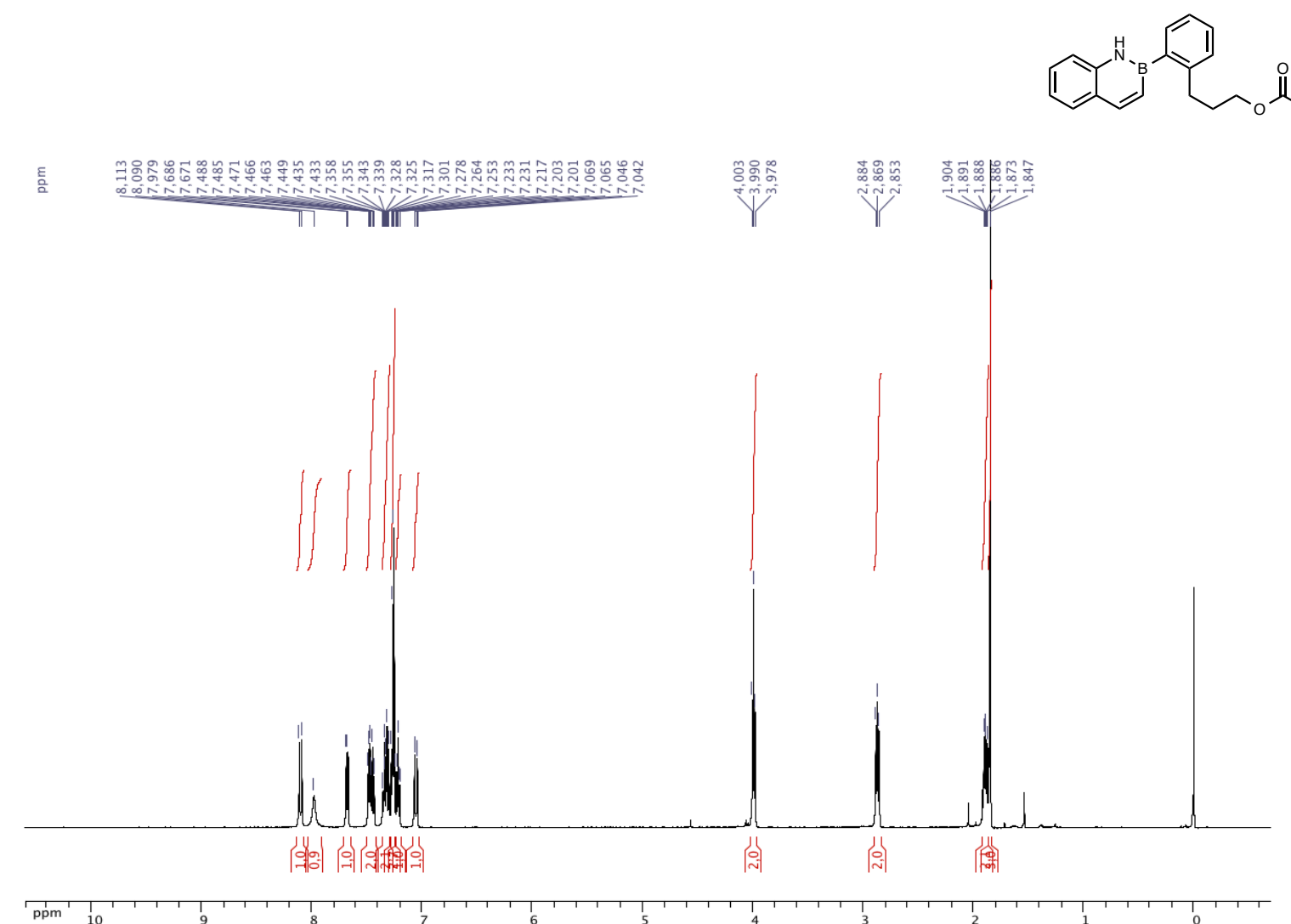

${ }^{1} \mathrm{H}$ NMR ( $\left.\mathrm{CDCl}_{3}, 500.4 \mathrm{MHz}\right)$ of 2-(2-(3-acetoxypropyl)phenyl)-2,1-borazanaphthalene (4c)

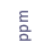

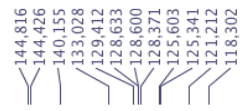

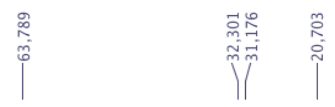
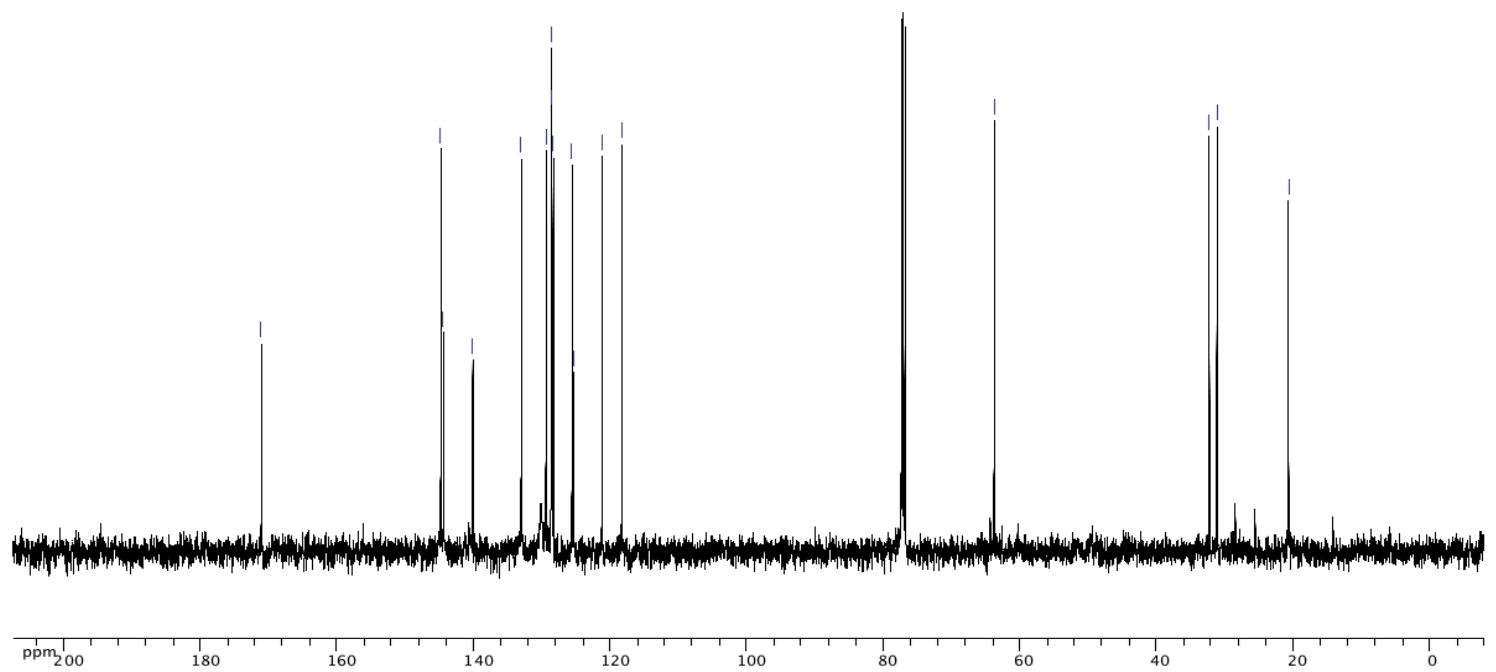

${ }^{13} \mathrm{C}\left\{{ }^{1} \mathrm{H}\right\}$ NMR $\left(\mathrm{CDCl}_{3}, 125.8 \mathrm{MHz}\right)$ of 2-(2-(3-acetoxypropyl)phenyl)-2,1-borazanaphthalene (4c) 


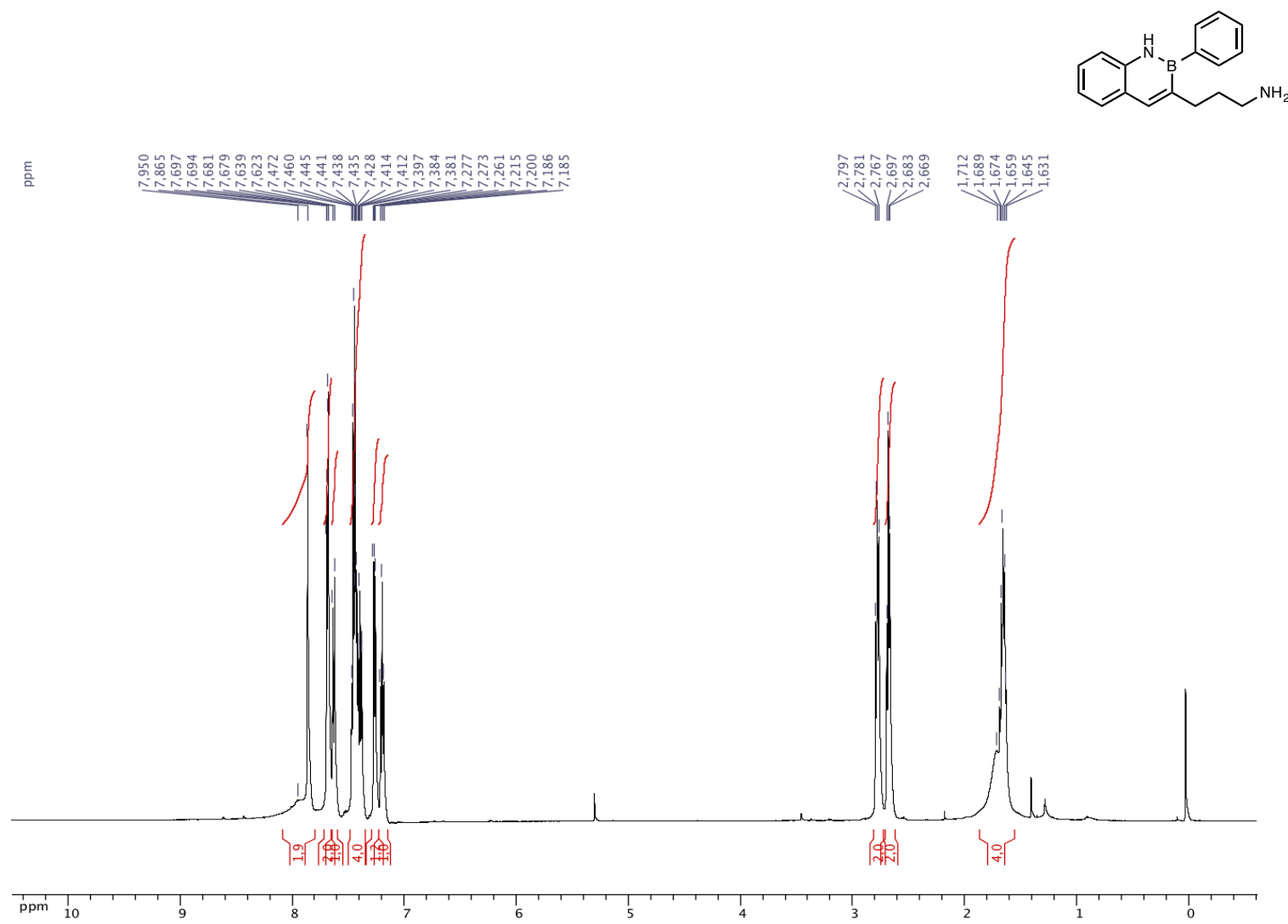

${ }^{1} \mathrm{H}$ NMR $\left(\mathrm{CDCl}_{3}, 500.4 \mathrm{MHz}\right)$ of 3-(3-aminopropyl)-2-phenyl-2,1-borazanaphthalene (5a)

言

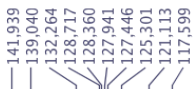

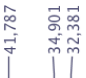
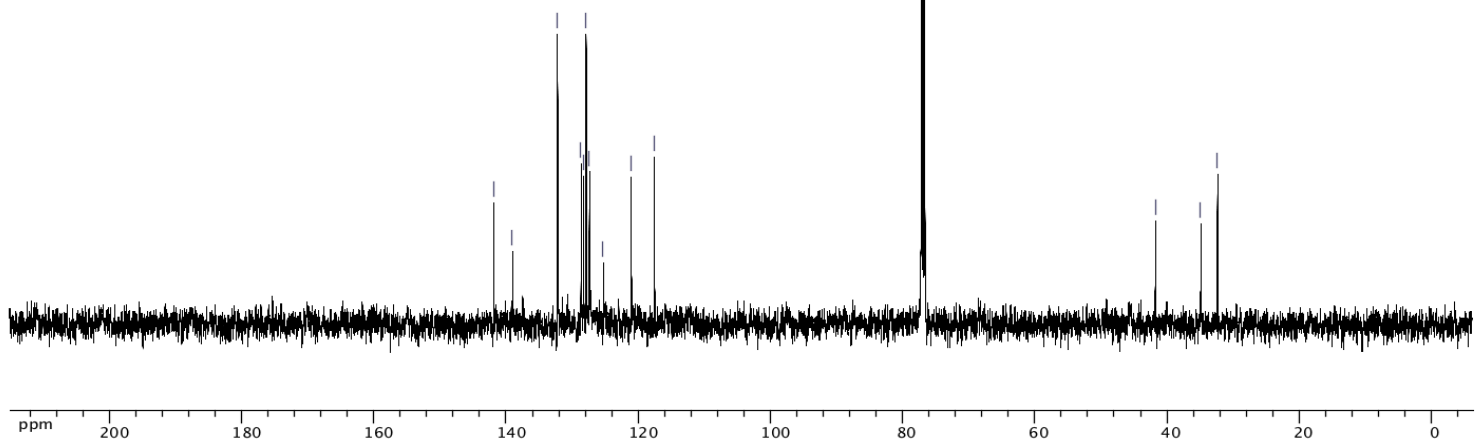

${ }^{13} \mathrm{C}\left\{{ }^{1} \mathrm{H}\right\}$ NMR $\left(\mathrm{CDCl}_{3}, 125.8 \mathrm{MHz}\right)$ of 3-(3-aminopropyl)-2-phenyl-2,1-borazanaphthalene (5a) 


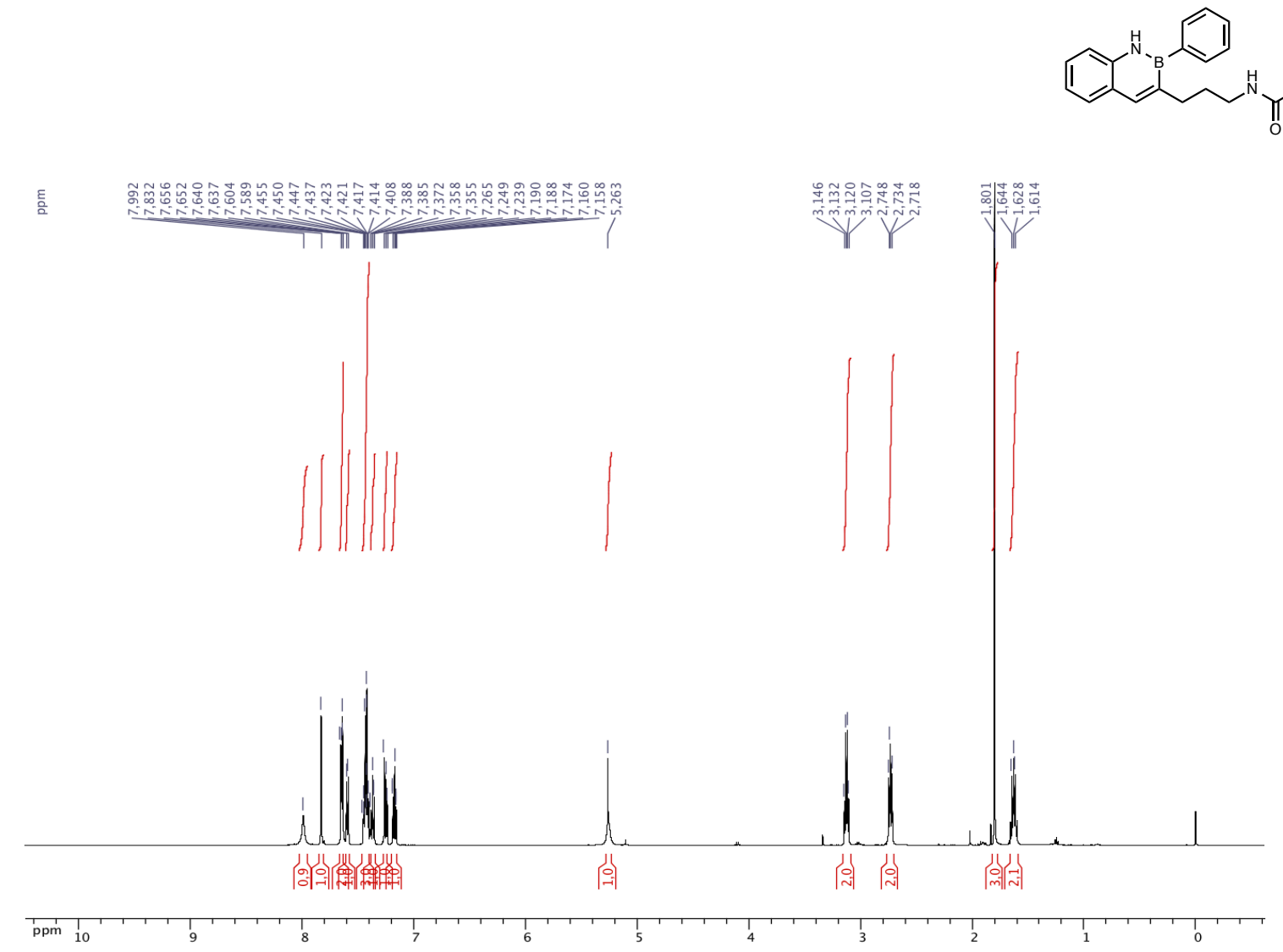

${ }^{1} \mathrm{H}$ NMR $\left(\mathrm{CDCl}_{3}, 500.4 \mathrm{MHz}\right)$ of 3-(3-acetamidopropyl)-2-phenyl-2,1-borazanaphthalene (5b) 言

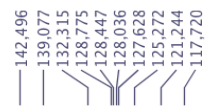

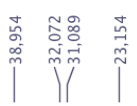
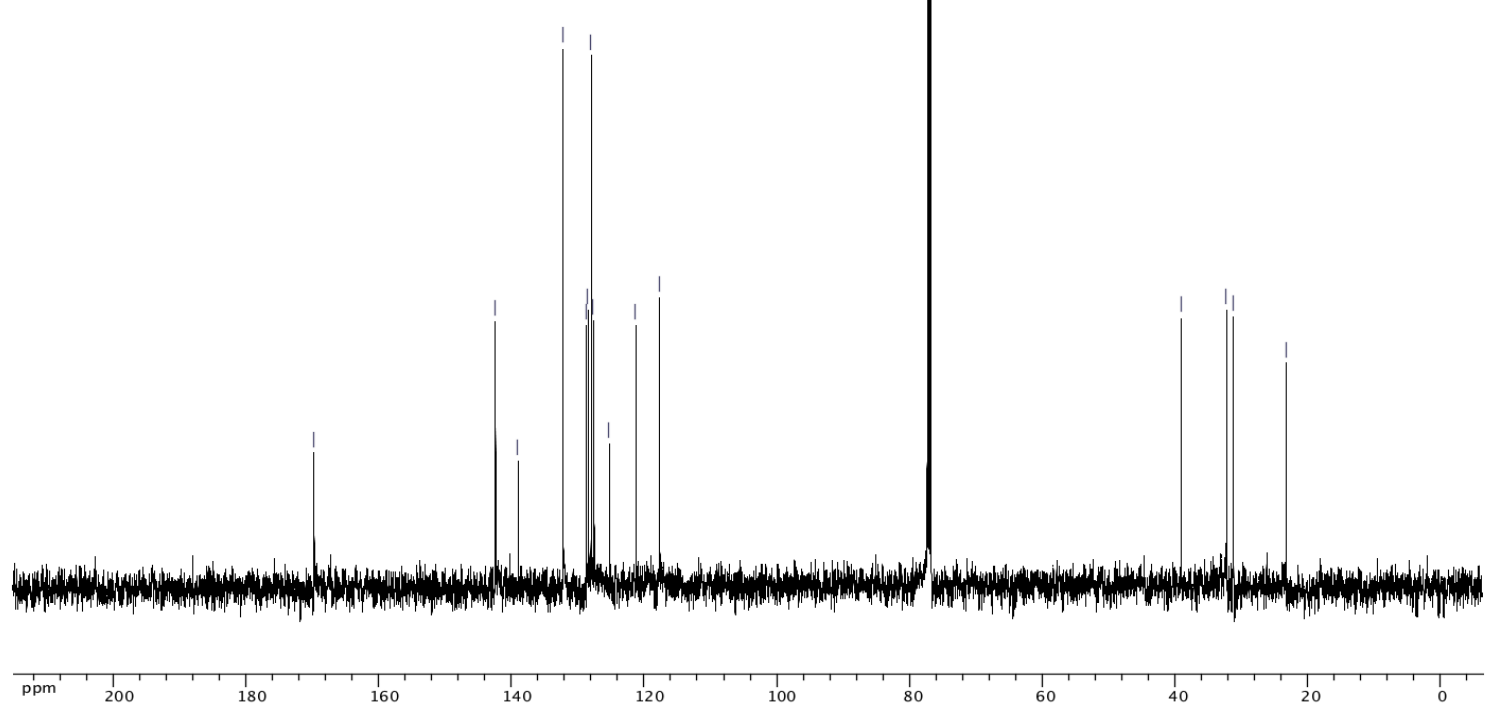

${ }^{13} \mathrm{C}\left\{{ }^{1} \mathrm{H}\right\}\left(\mathrm{CDCl}_{3}, 125.8 \mathrm{MHz}\right)$ of 3-(3-acetamidopropyl)-2-phenyl-2,1-borazanaphthalene (5b) 


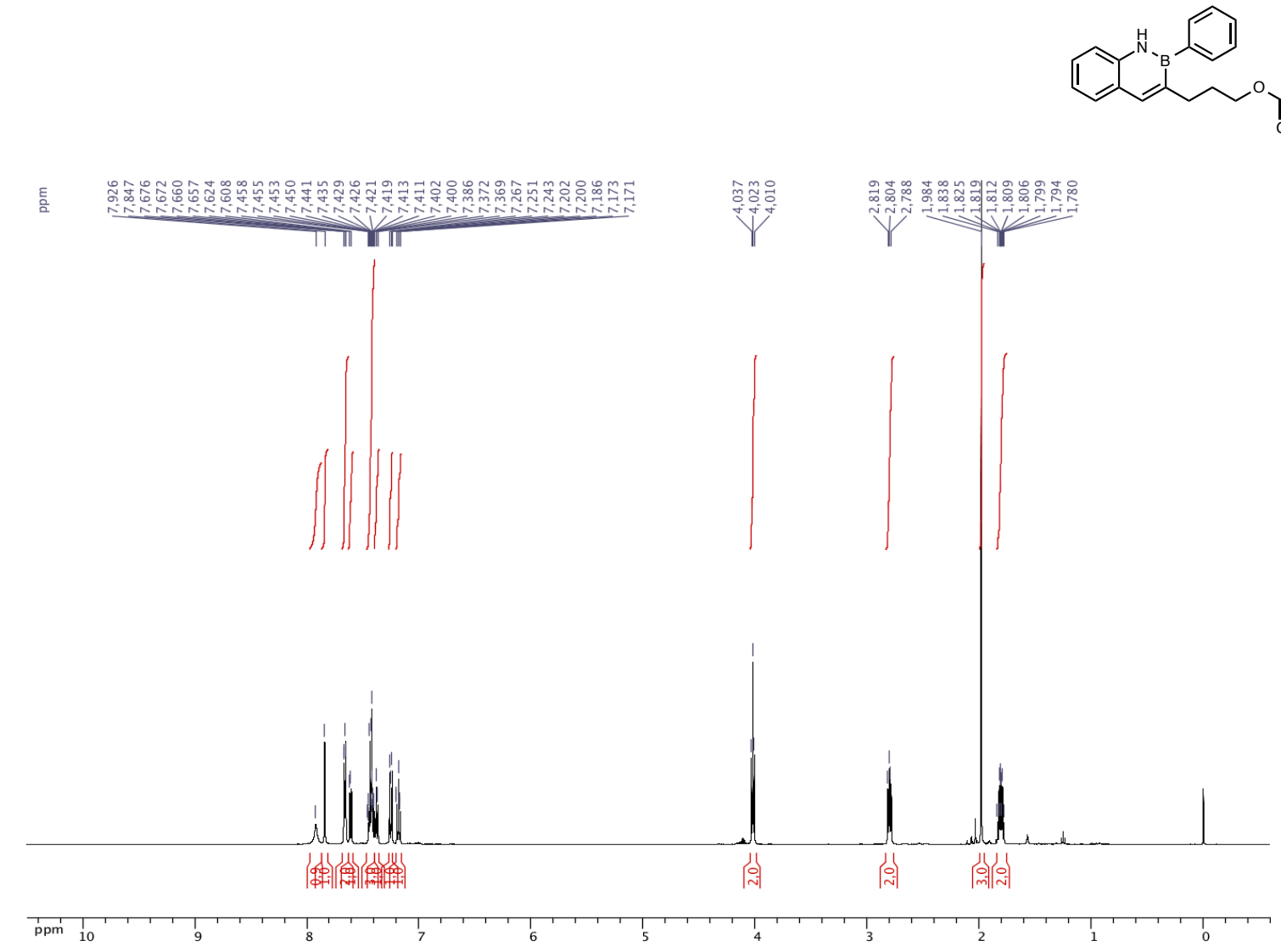

${ }^{1} \mathrm{H} \mathrm{NMR}\left(\mathrm{CDCl}_{3}, 500.4 \mathrm{MHz}\right)$ of 3-(3-acetoxypropyl)-2-phenyl-2,1-borazanaphthalene (5c)

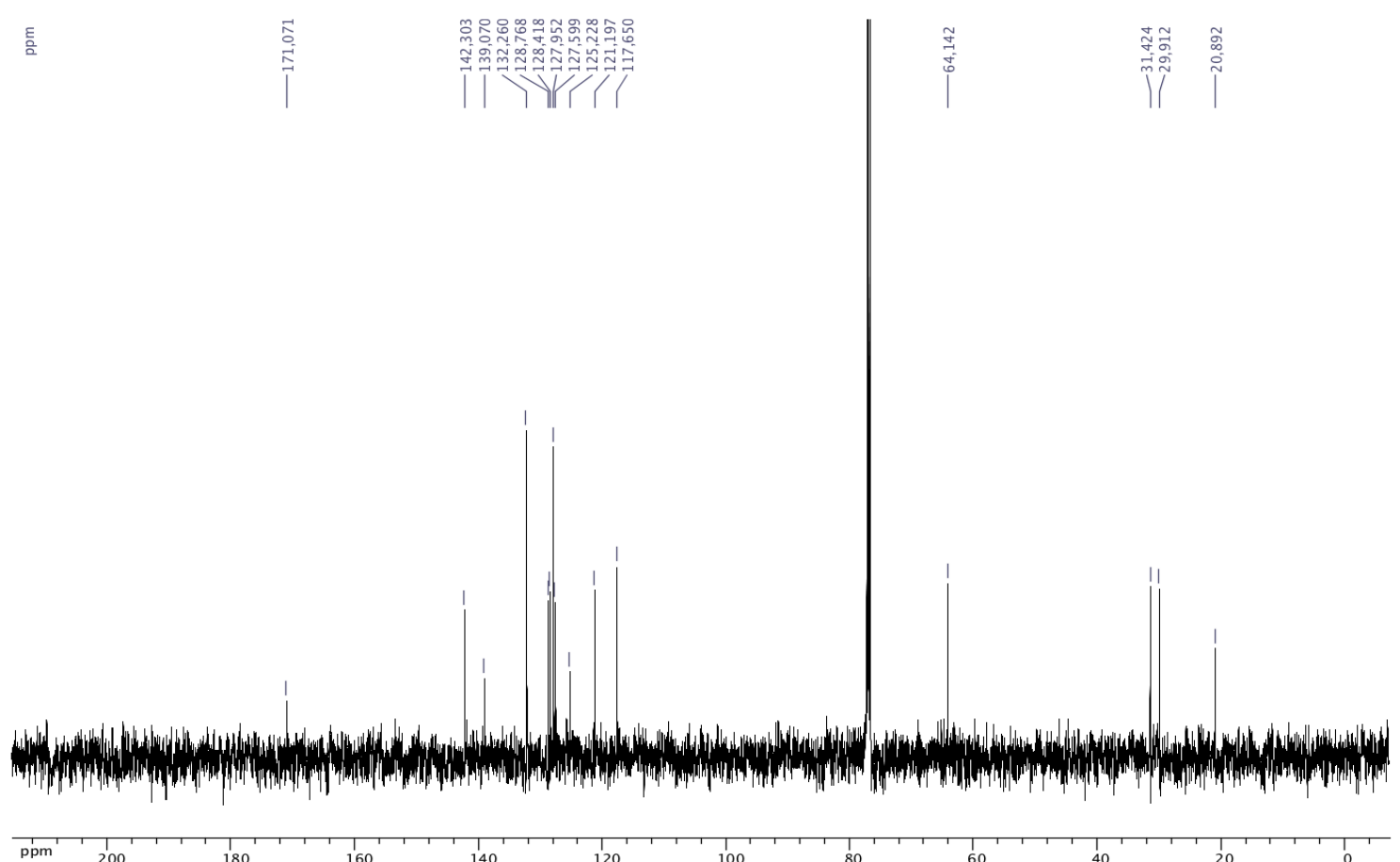

${ }^{13} \mathrm{C}\left\{{ }^{1} \mathrm{H}\right\}$ NMR $\left(\mathrm{CDCl}_{3}, 125.8 \mathrm{MHz}\right)$ of 3-(3-acetoxypropyl)-2-phenyl-2,1-borazanaphthalene (5c) 


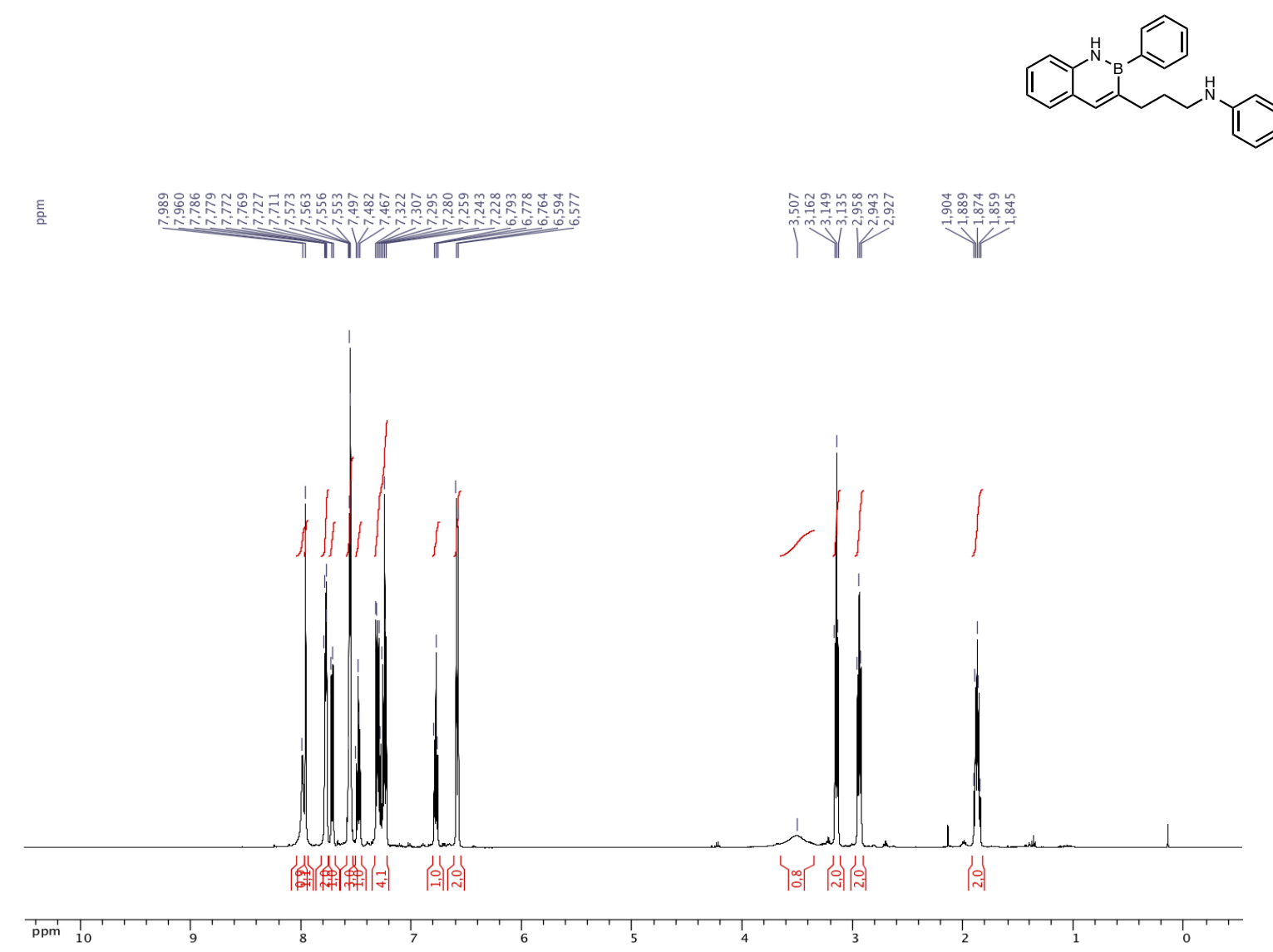

${ }^{1} \mathrm{H}$ NMR $\left(\mathrm{CDCl}_{3}, 500.4 \mathrm{MHz}\right)$ of 3-(3-(phenylamino)propyl)-2-phenyl-2,1-borazanaphthalene (5d) 言
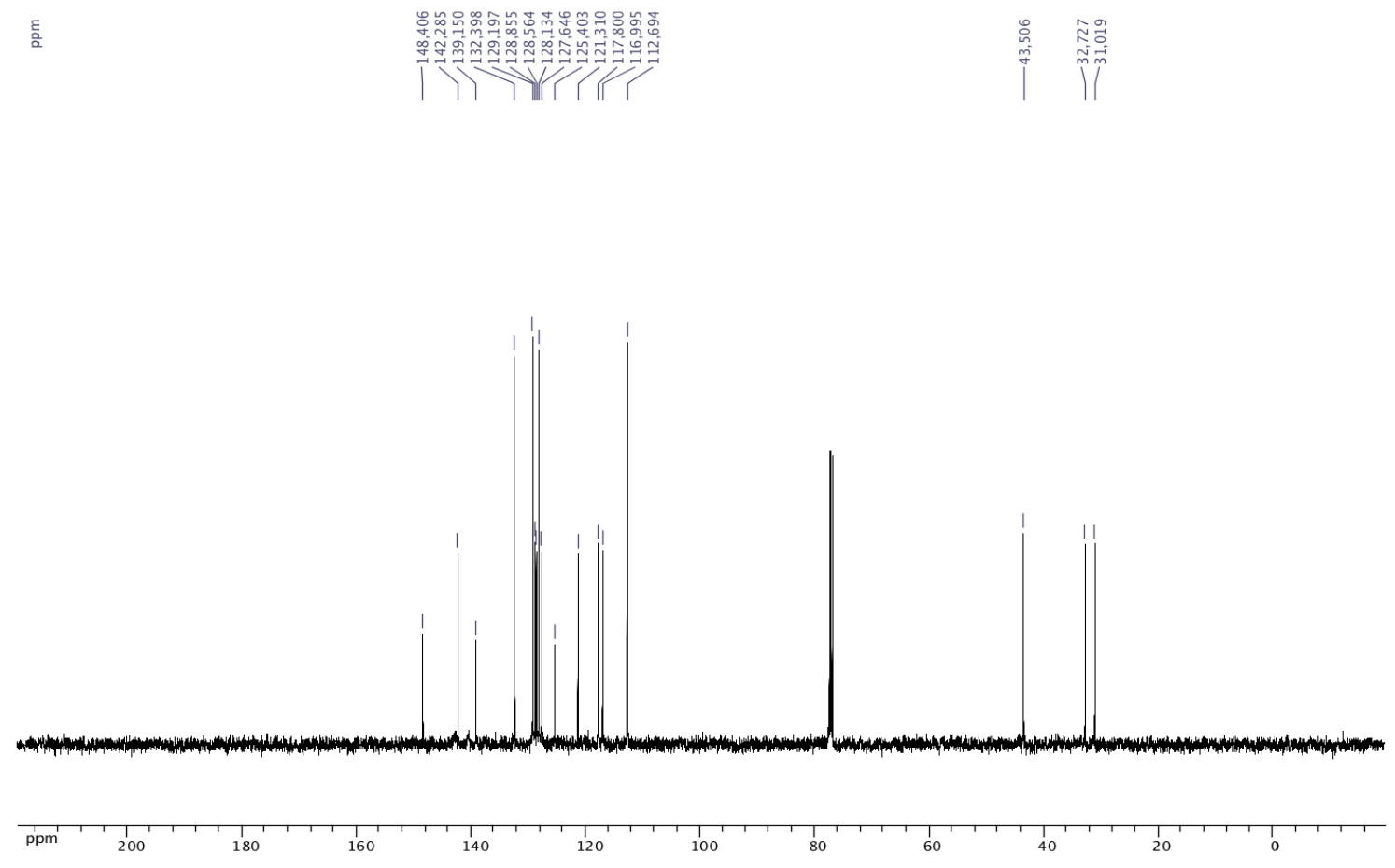

${ }^{13} \mathrm{C}\left\{{ }^{1} \mathrm{H}\right\}$ NMR $\left(\mathrm{CDCl}_{3}, 125.8 \mathrm{MHz}\right)$ of 3-(3-(phenylamino)propyl)-2-phenyl-2,1-borazanaphthalene (5d) 


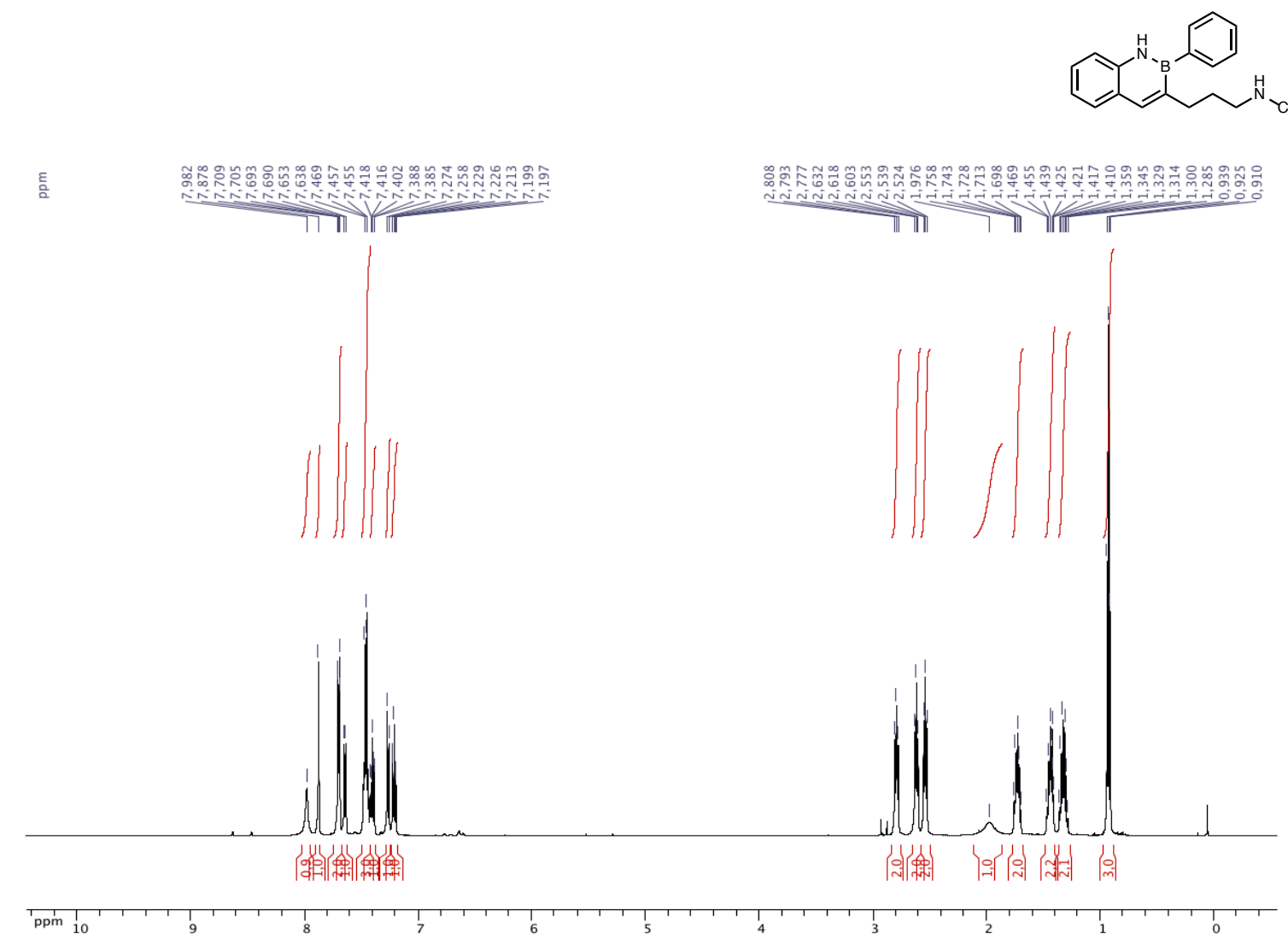

${ }^{1} \mathrm{H} \mathrm{NMR}\left(\mathrm{CDCl}_{3}, 500.4 \mathrm{MHz}\right.$ ) of 3-(3-(butylamino)propyl)-2-phenyl-2,1-borazanaphthalene (5e)
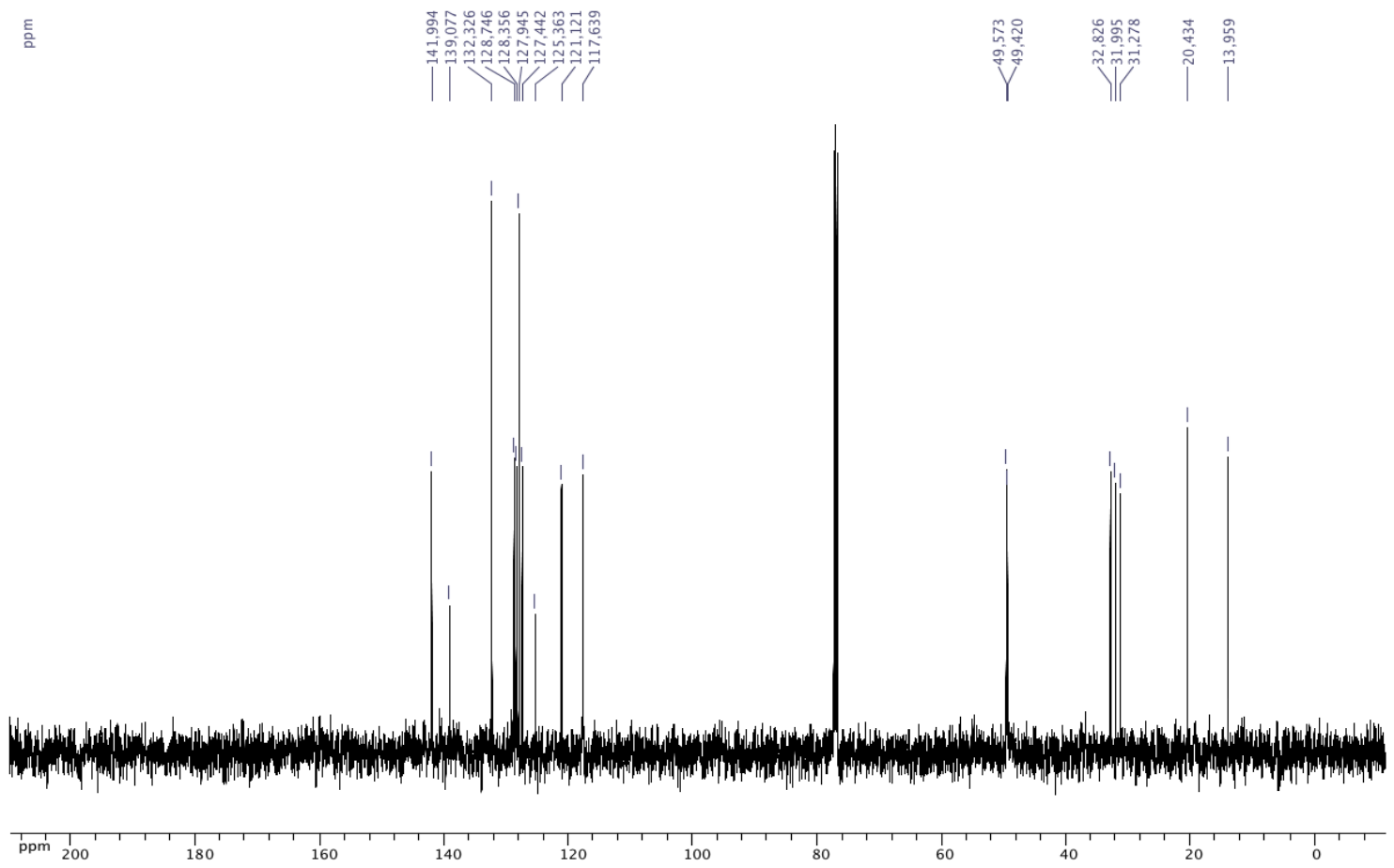

${ }^{13} \mathrm{C}\left\{{ }^{1} \mathrm{H}\right\}$ NMR $\left(\mathrm{CDCl}_{3}, 125.8 \mathrm{MHz}\right)$ of 3-(3-(butylamino)propyl)-2-phenyl-2,1-borazanaphthalene (5e) 


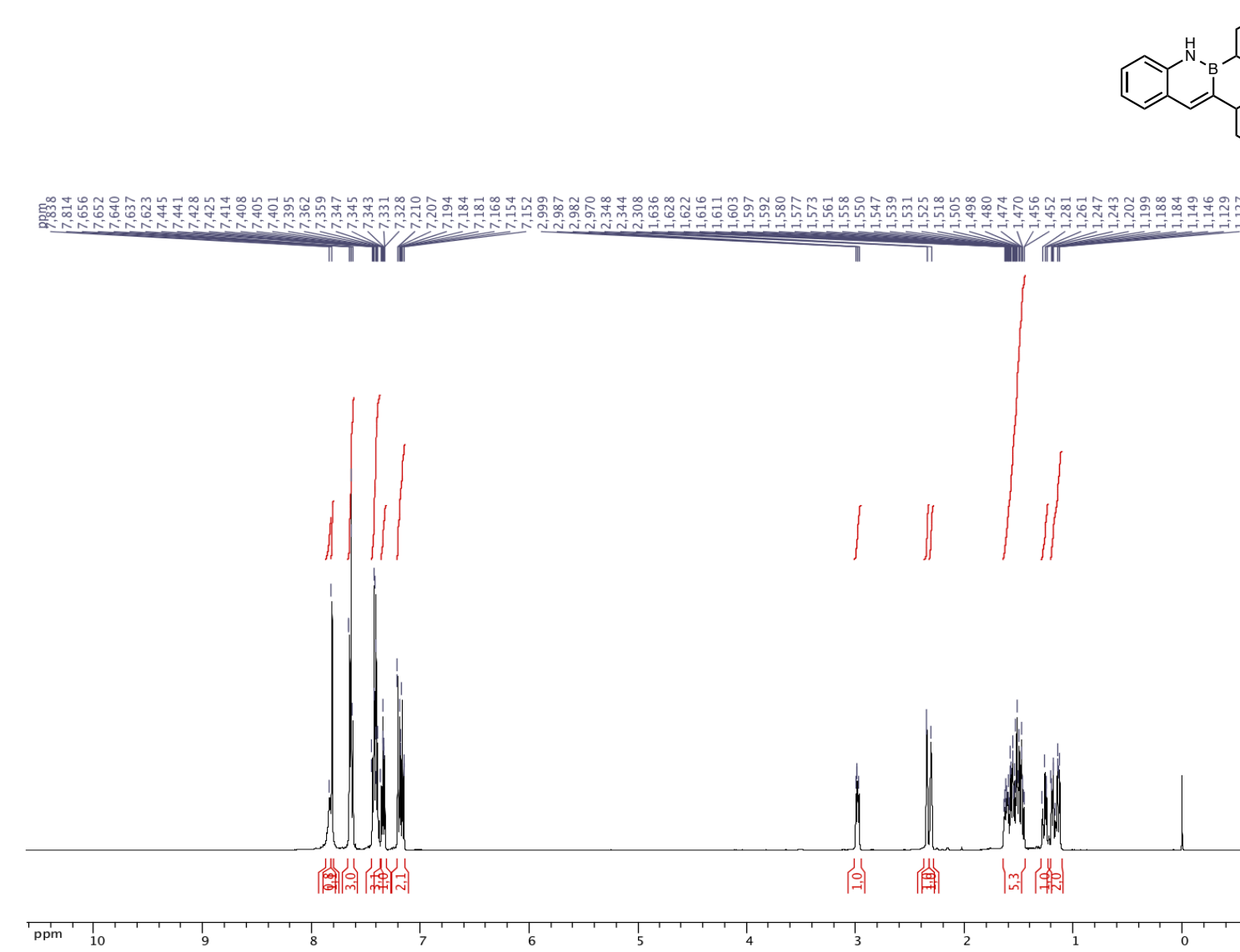

${ }^{1} \mathrm{H}$ NMR ( $\left.\mathrm{CDCl}_{3}, 500.4 \mathrm{MHz}\right)$ of 3-(exo-bicyclo[2.2.1]heptan-2-yl)-2-phenyl-2,1-borazanaphthalene (5f) 言
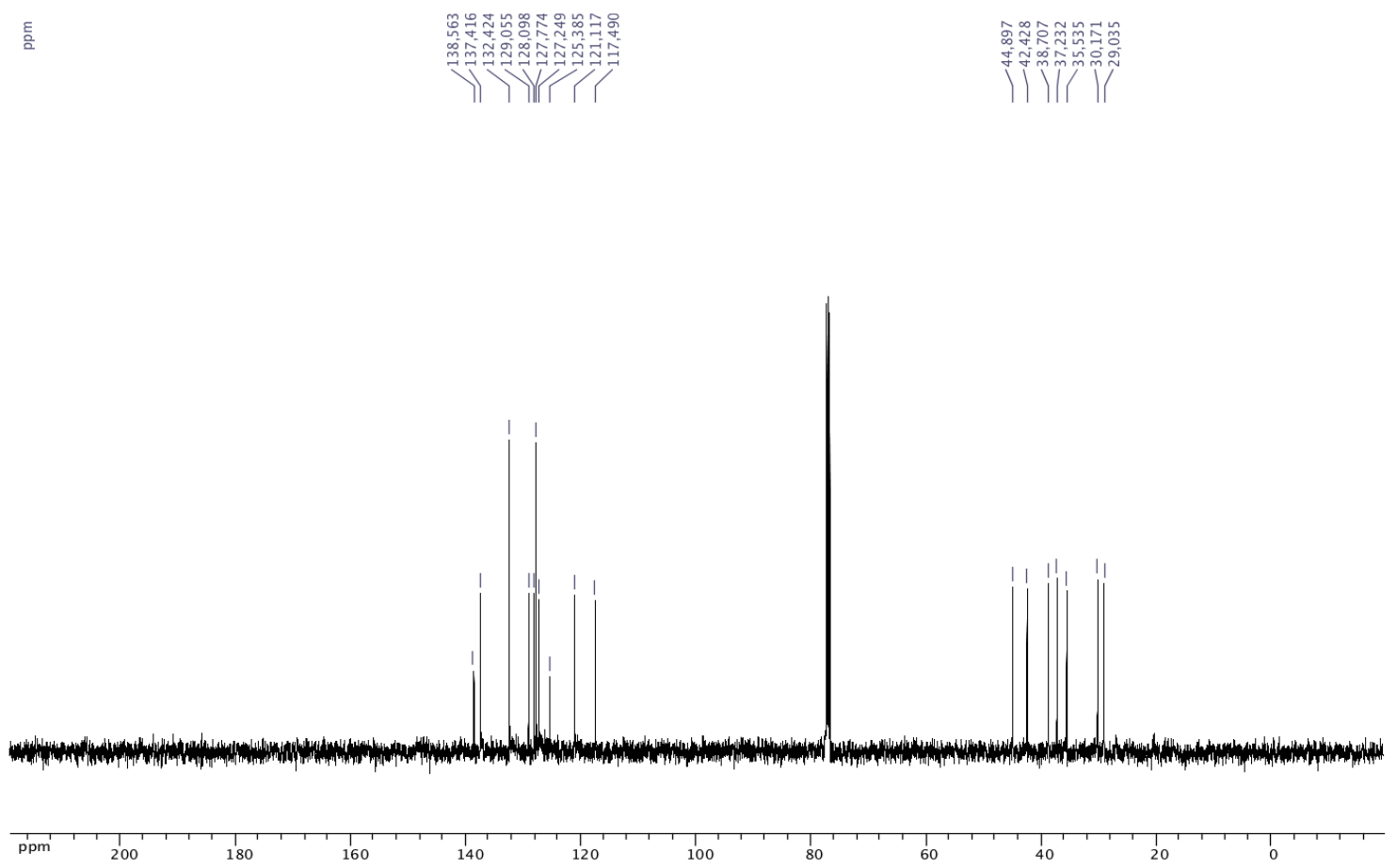

${ }^{13} \mathrm{C}\left\{{ }^{1} \mathrm{H}\right\} \mathrm{NMR}\left(\mathrm{CDCl}_{3}, 125.8 \mathrm{MHz}\right)$ of 3-(exo-bicyclo[2.2.1]heptan-2-yl)-2-phenyl-2,1-borazanaphthalene (5f) 

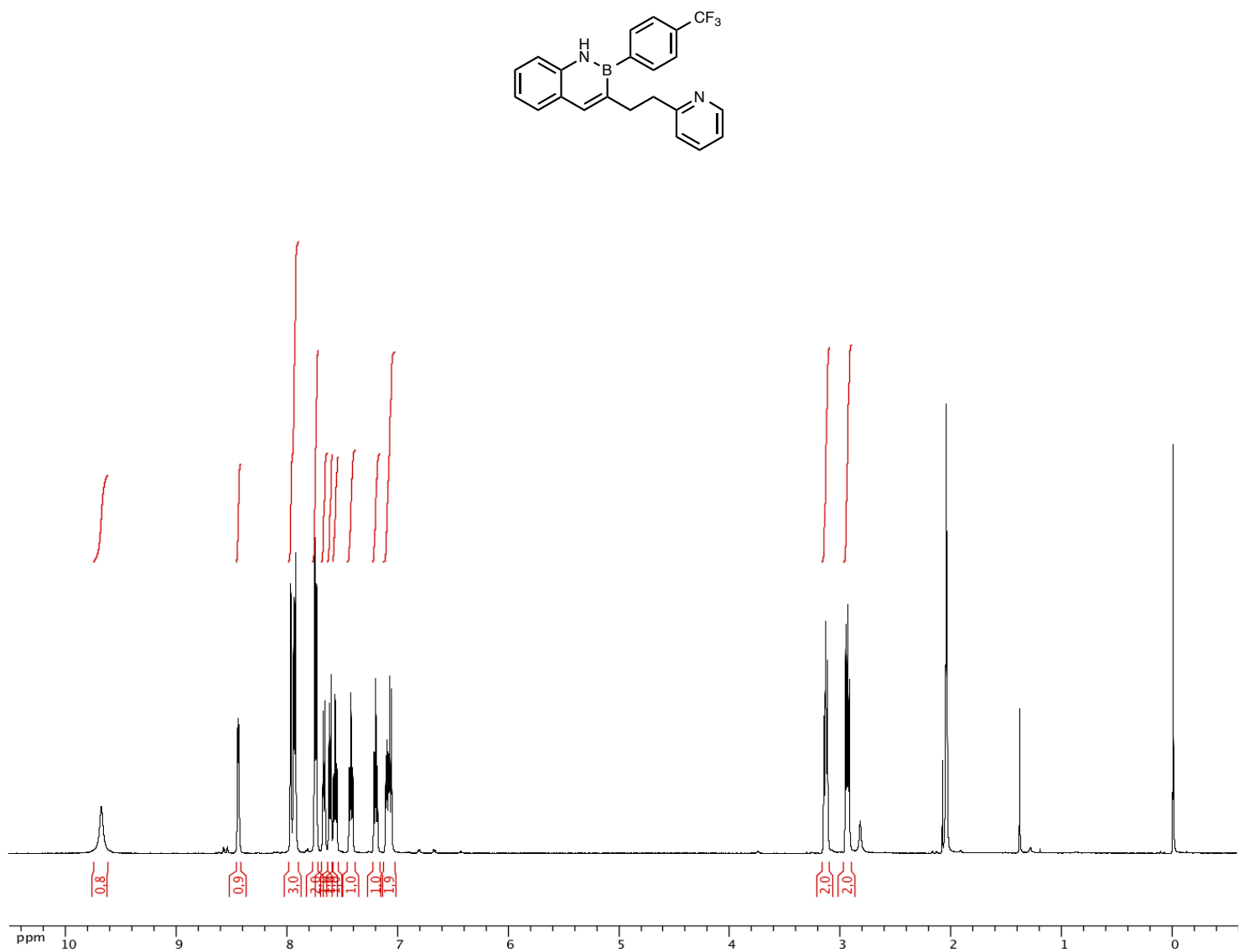

${ }^{1} \mathrm{H}$ NMR (acetone- $d_{6}, 500.4 \mathrm{MHz}$ ) of 3-(2-pyridin-2-yl)ethyl)-2-(4-trifluoromethylphenyl)-2,1borazanaphthalene (6)
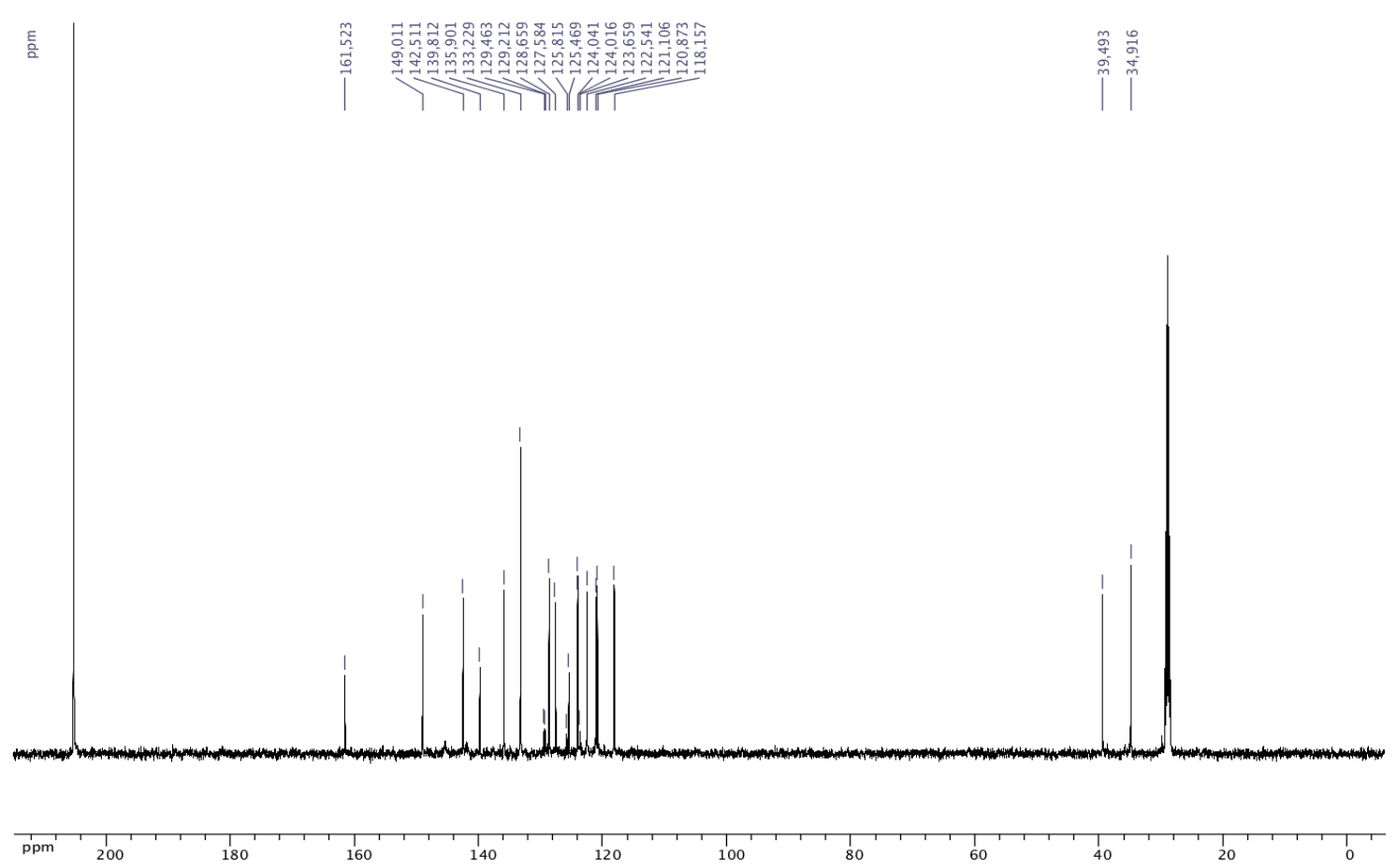

${ }^{13} \mathrm{C}\left\{{ }^{1} \mathrm{H}\right\}$ NMR (acetone- $d_{6}, 125.8 \mathrm{MHz}$ ) of 3-(2-pyridin-2-yl)ethyl)-2-(4-trifluoromethylphenyl)-2,1borazanaphthalene $(\mathbf{6})$ 


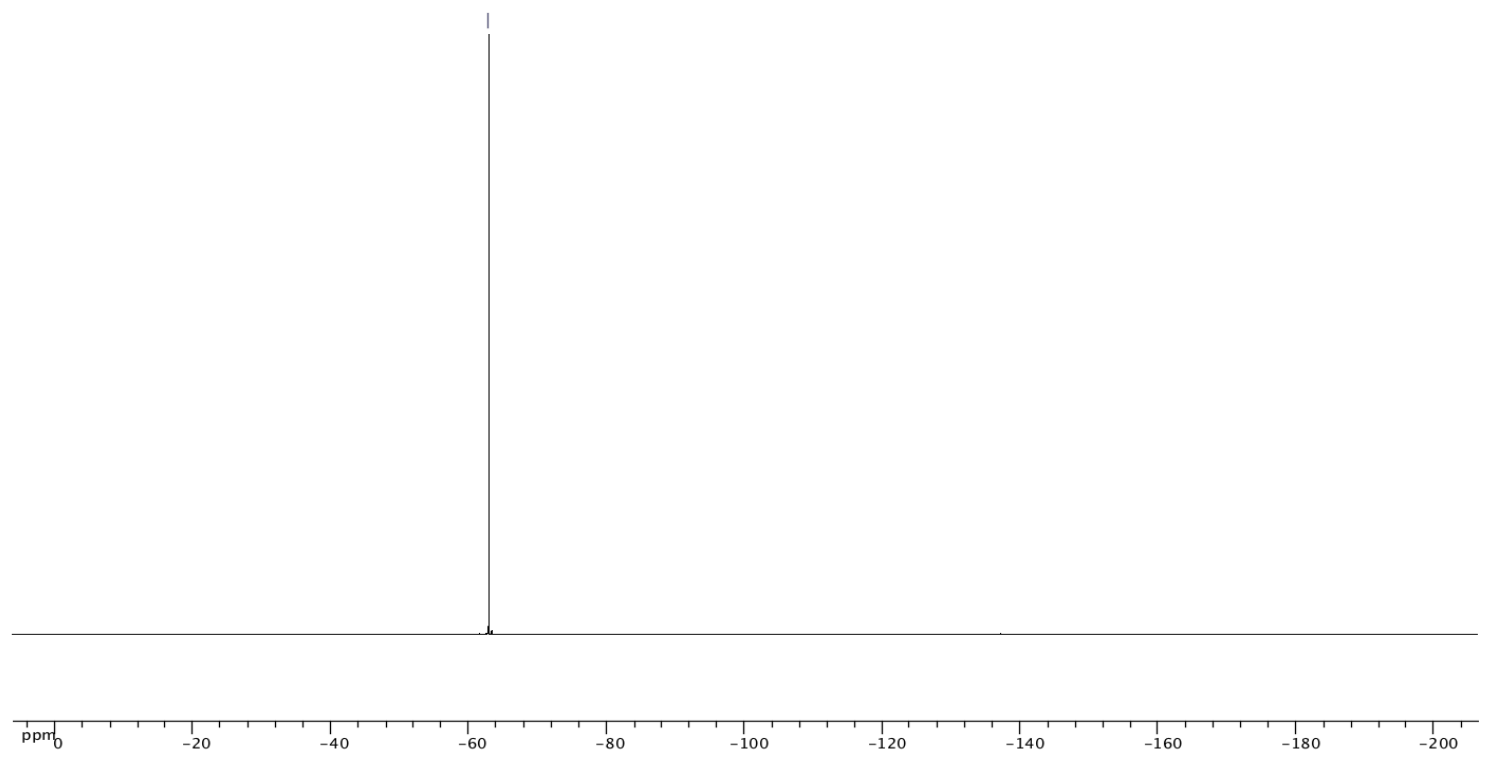

${ }^{19} \mathrm{~F}\left\{{ }^{1} \mathrm{H}\right\}$ NMR (acetone- $d_{6}, 470.8 \mathrm{MHz}$ ) of 3-(2-pyridin-2-yl)ethyl)-2-(4-trifluoromethylphenyl)-2,1borazanaphthalene (6) 


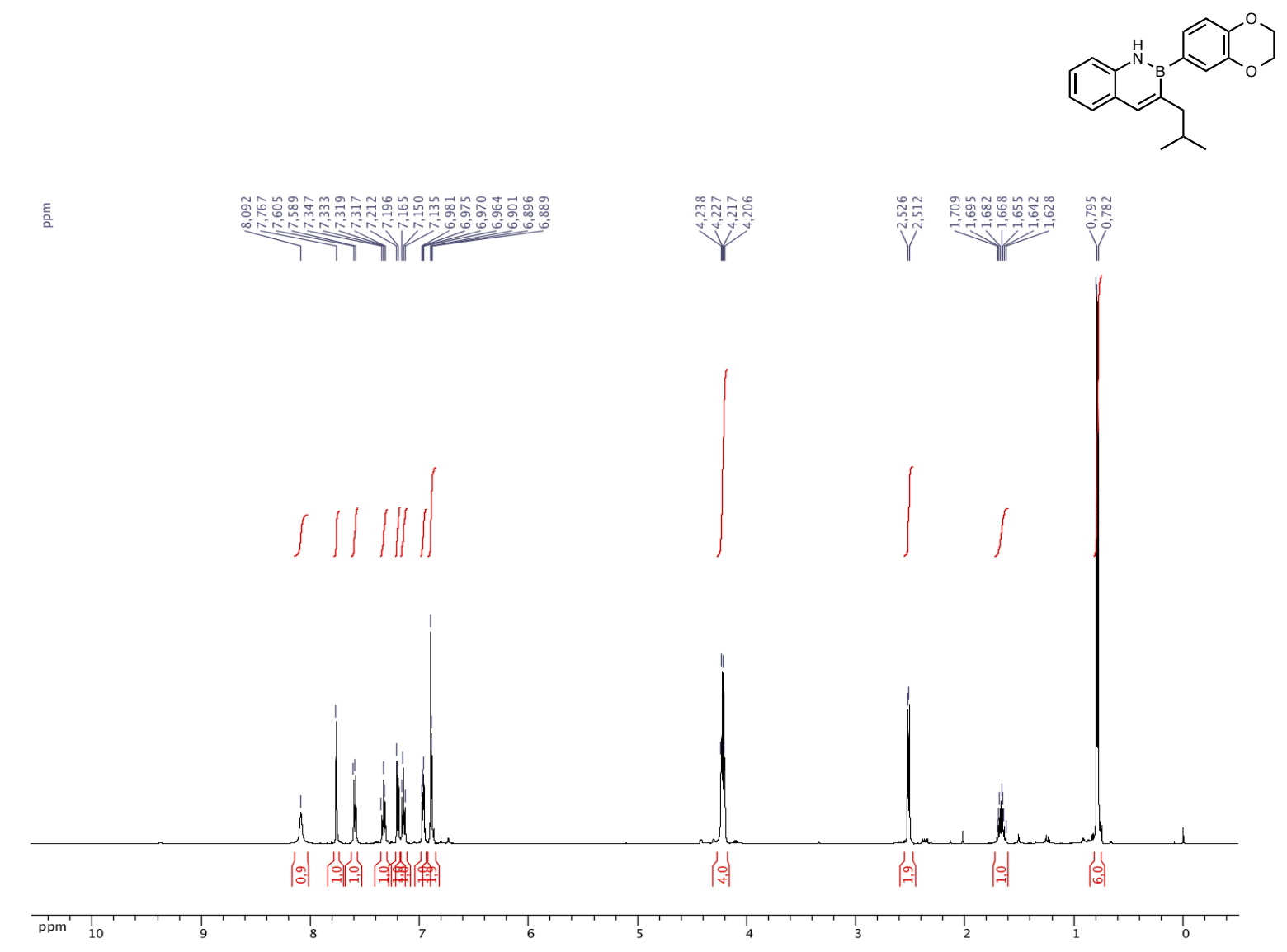

${ }^{1} \mathrm{H}$ NMR ( $\mathrm{CDCl}_{3}, 500.4 \mathrm{MHz}$ ) of 3-(isobutyl)-2-(2,3-dihydro-1,4-benzodioxin-6-yl)-2,1-borazanaphthalene (7)
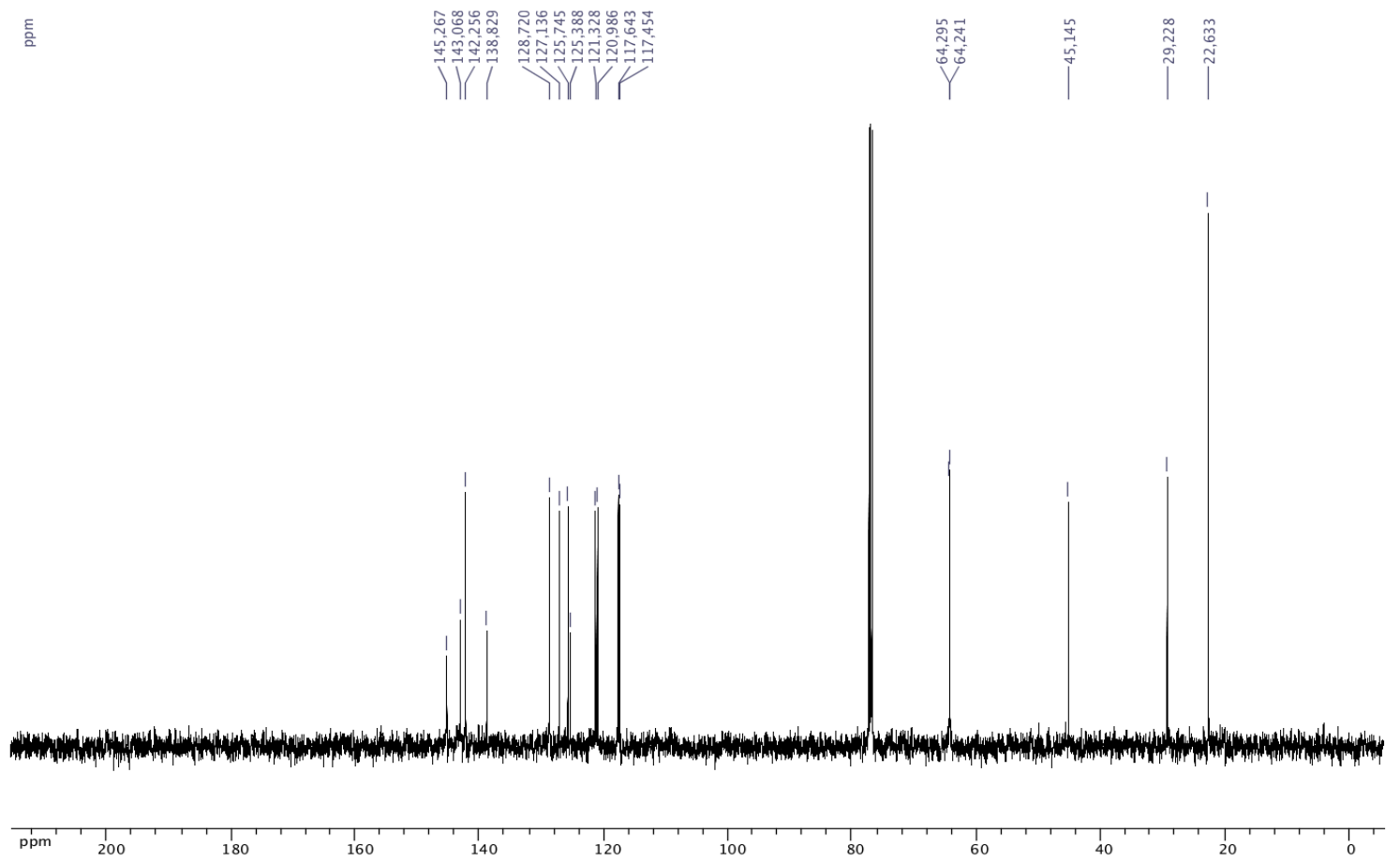

${ }^{13} \mathrm{C}\left\{{ }^{1} \mathrm{H}\right\}$ NMR ( $\left.\mathrm{CDCl}_{3}, 125.8 \mathrm{MHz}\right)$ of 3-(isobutyl)-2-(2,3-dihydro-1,4-benzodioxin-6-yl)-2,1borazanaphthalene (7) 

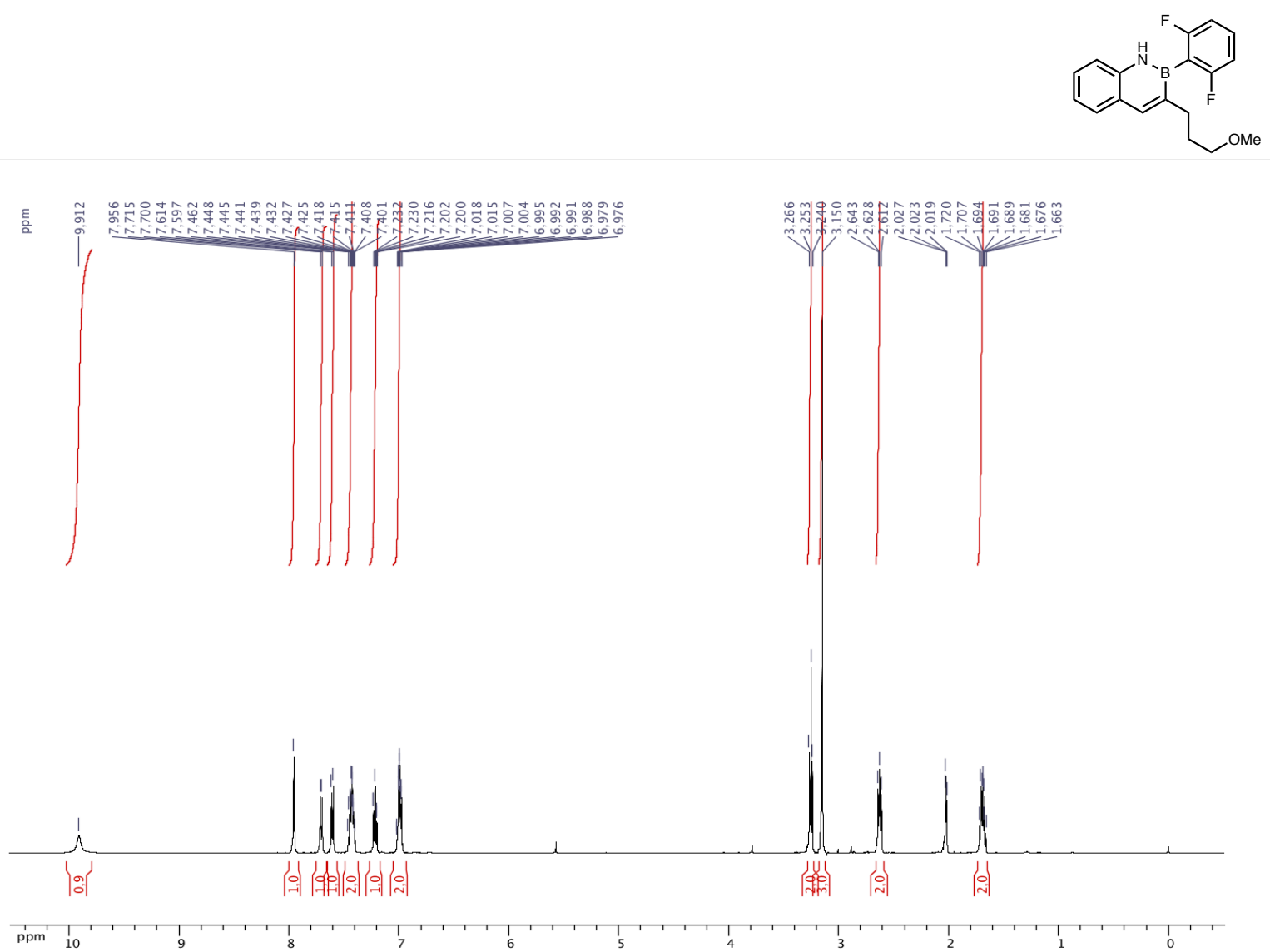

${ }^{1} \mathrm{H}$ NMR (acetone- $d_{6}, 500.4 \mathrm{MHz}$ ) of 3-(3-methoxypropyl)-2-(2,6-difluorophenyl)-2,1-borazanaphthalene (8)

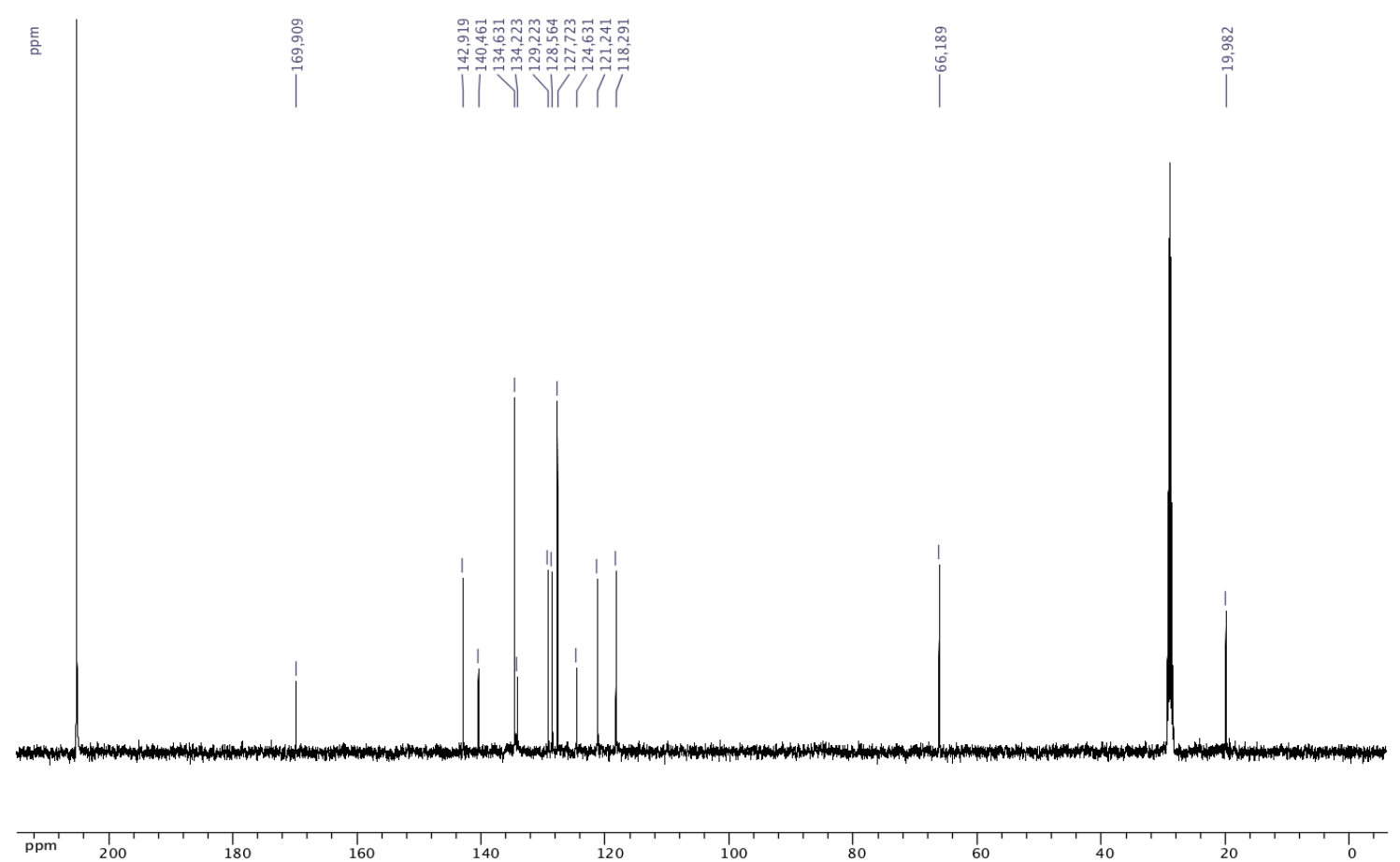

${ }^{13} \mathrm{C}\left\{{ }^{1} \mathrm{H}\right\}$ NMR (acetone- $d_{6}, 125.8 \mathrm{MHz}$ ) of 3-(3-methoxypropyl)-2-(2,6-difluorophenyl)-2,1-borazanaphthalene (8) 


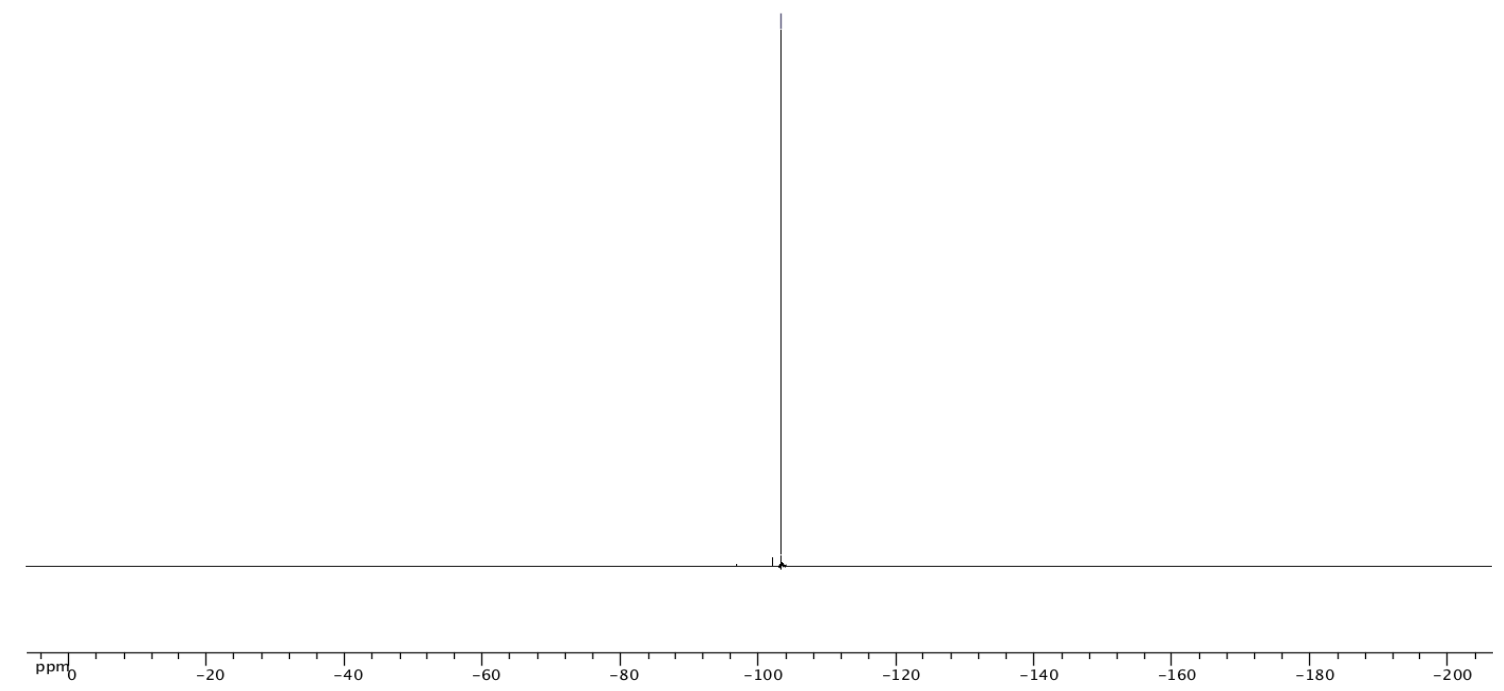

${ }^{19} \mathrm{~F}\left\{{ }^{1} \mathrm{H}\right\}$ NMR (acetone- $d_{6}, 470.8 \mathrm{MHz}$ ) of 3-(3-methoxypropyl)-2-(2,6-difluorophenyl)-2,1-borazanaphthalene (8) 


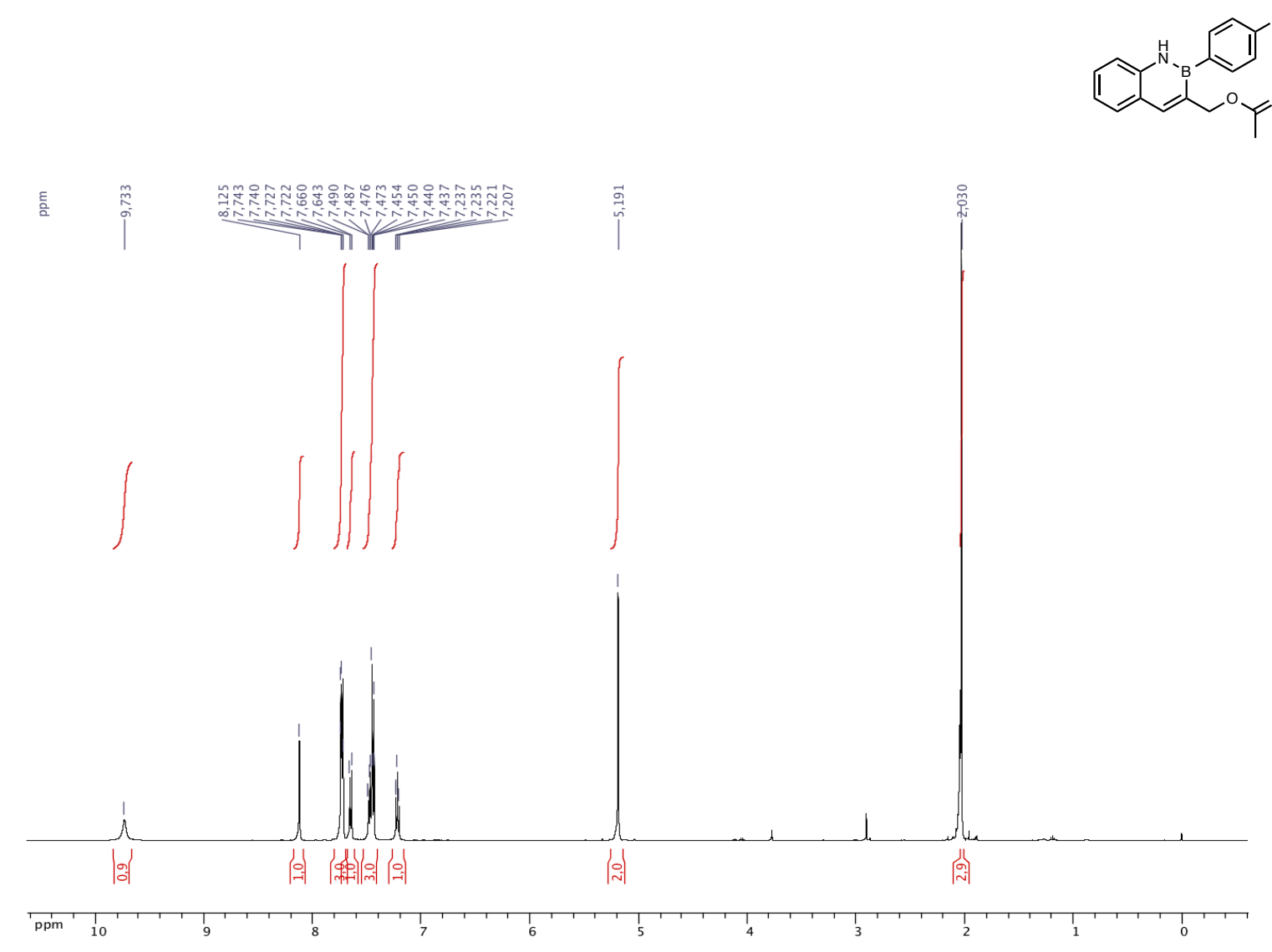

${ }^{1} \mathrm{H}$ NMR (acetone- $d_{6}, 500.4 \mathrm{MHz}$ ) of 3-(acetoxymethyl)-2-(4-chlorophenyl)-2,1-borazanaphthalene (9)

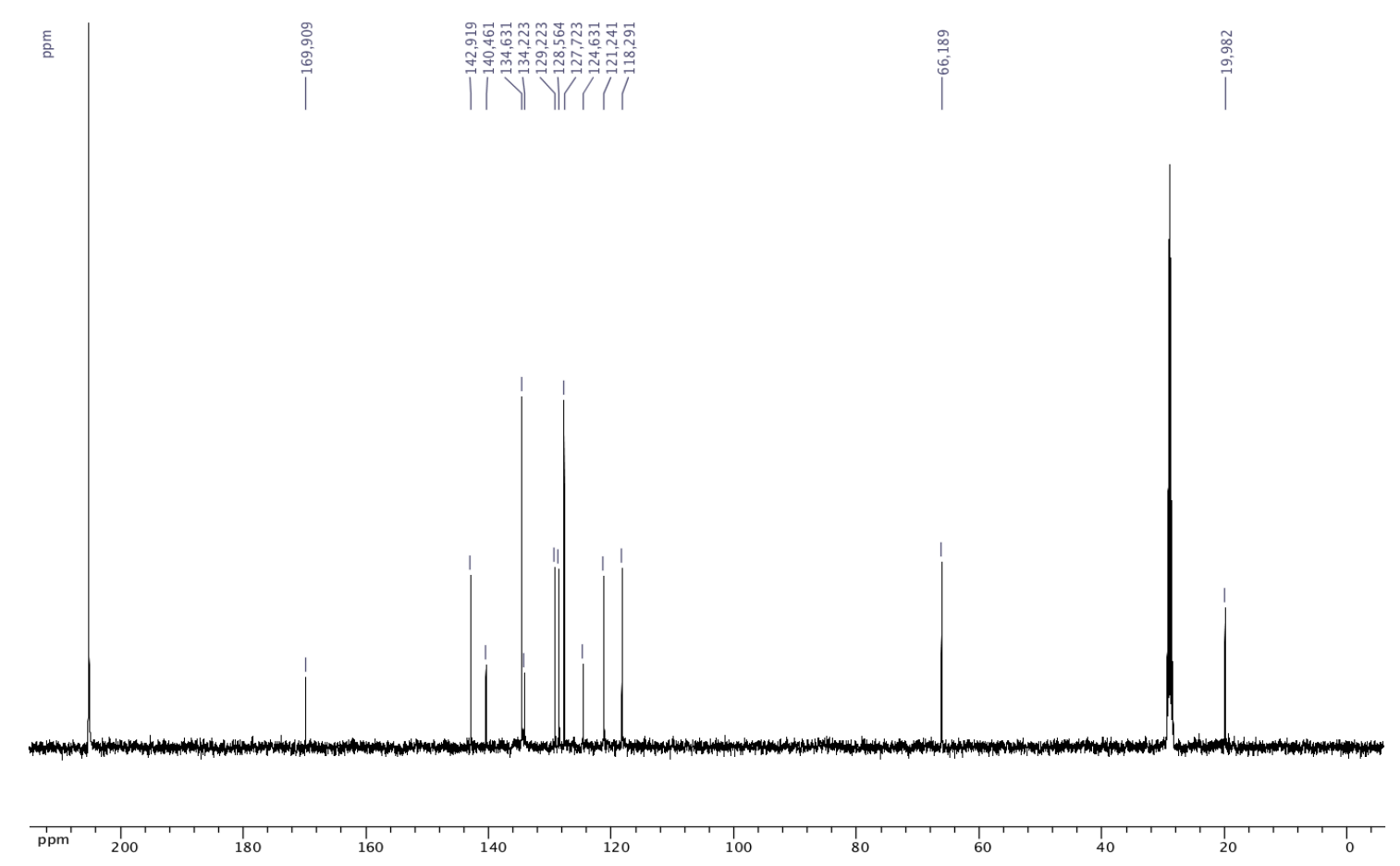

${ }^{13} \mathrm{C}\left\{{ }^{1} \mathrm{H}\right\}$ NMR (acetone- $d_{6}, 125.8 \mathrm{MHz}$ ) of 3-(acetoxymethyl)-2-(4-chlorophenyl)-2,1-borazanaphthalene (9) 


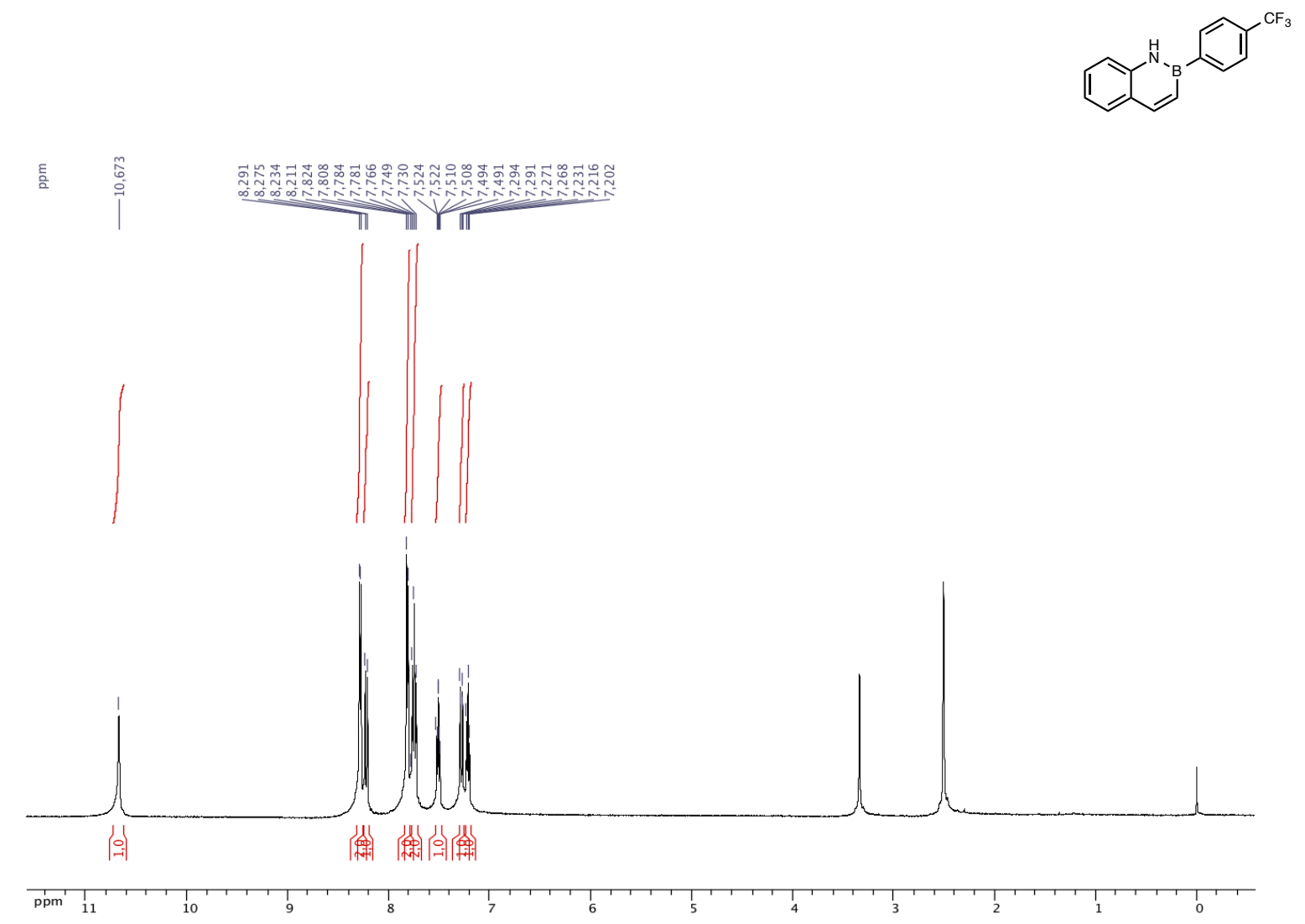

${ }^{1} \mathrm{H}$ NMR (DMSO- $d_{6}, 500.4 \mathrm{MHz}$ ) of 2-(4-(trifluoromethyl)phenyl)-2,1-borazanaphthalene (10a)
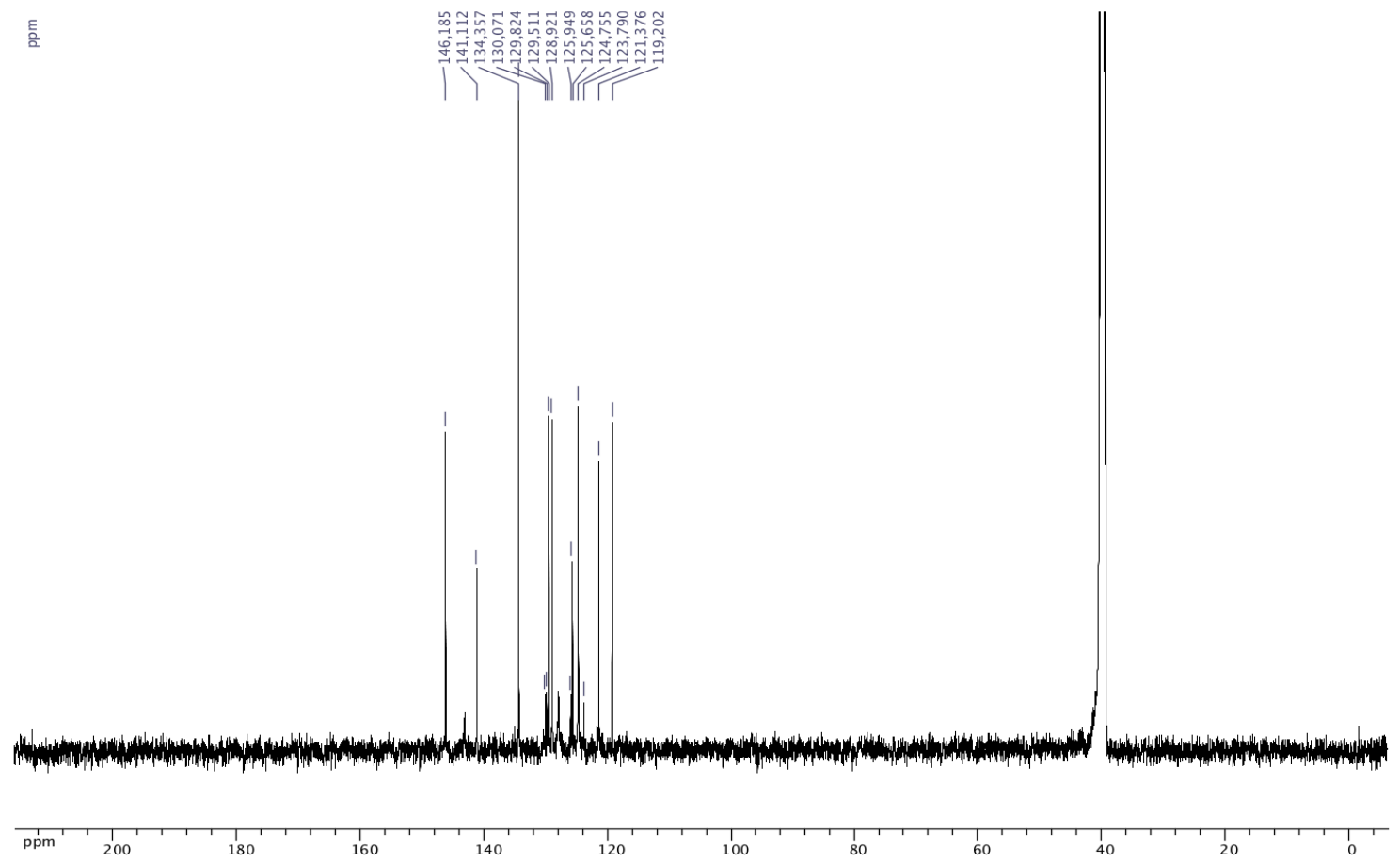

${ }^{13} \mathrm{C}\left\{{ }^{1} \mathrm{H}\right\}$ NMR (DMSO- $\left.d_{6}, 125.8 \mathrm{MHz}\right)$ of 2-(4-(trifluoromethyl)phenyl)-2,1-borazanaphthalene (10a) 


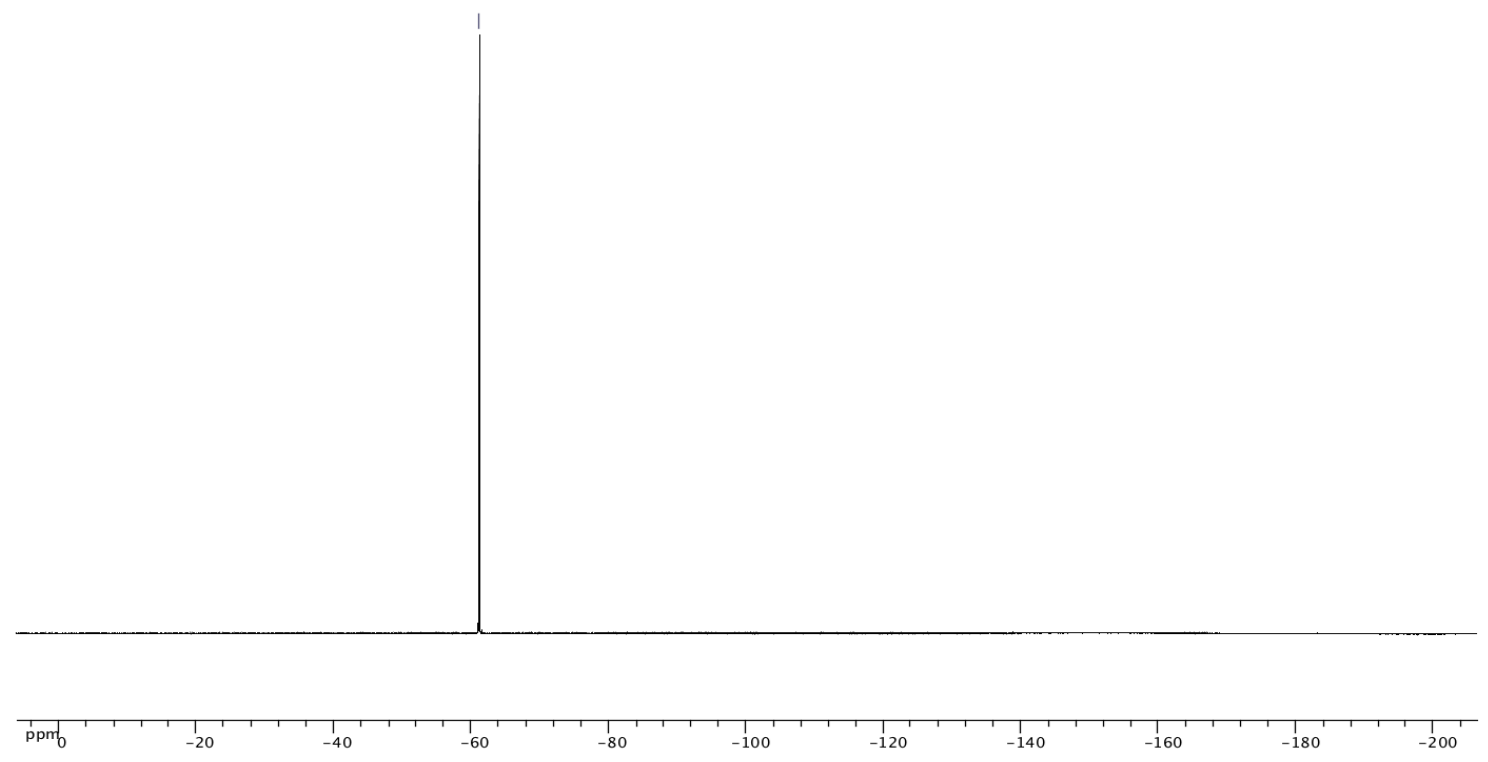

${ }^{19} \mathrm{~F}\left\{{ }^{1} \mathrm{H}\right\}$ NMR (DMSO- $d_{6}, 470.8 \mathrm{MHz}$ ) of of 2-(4-(trifluoromethyl)phenyl)-2,1-borazanaphthalene (10a) 


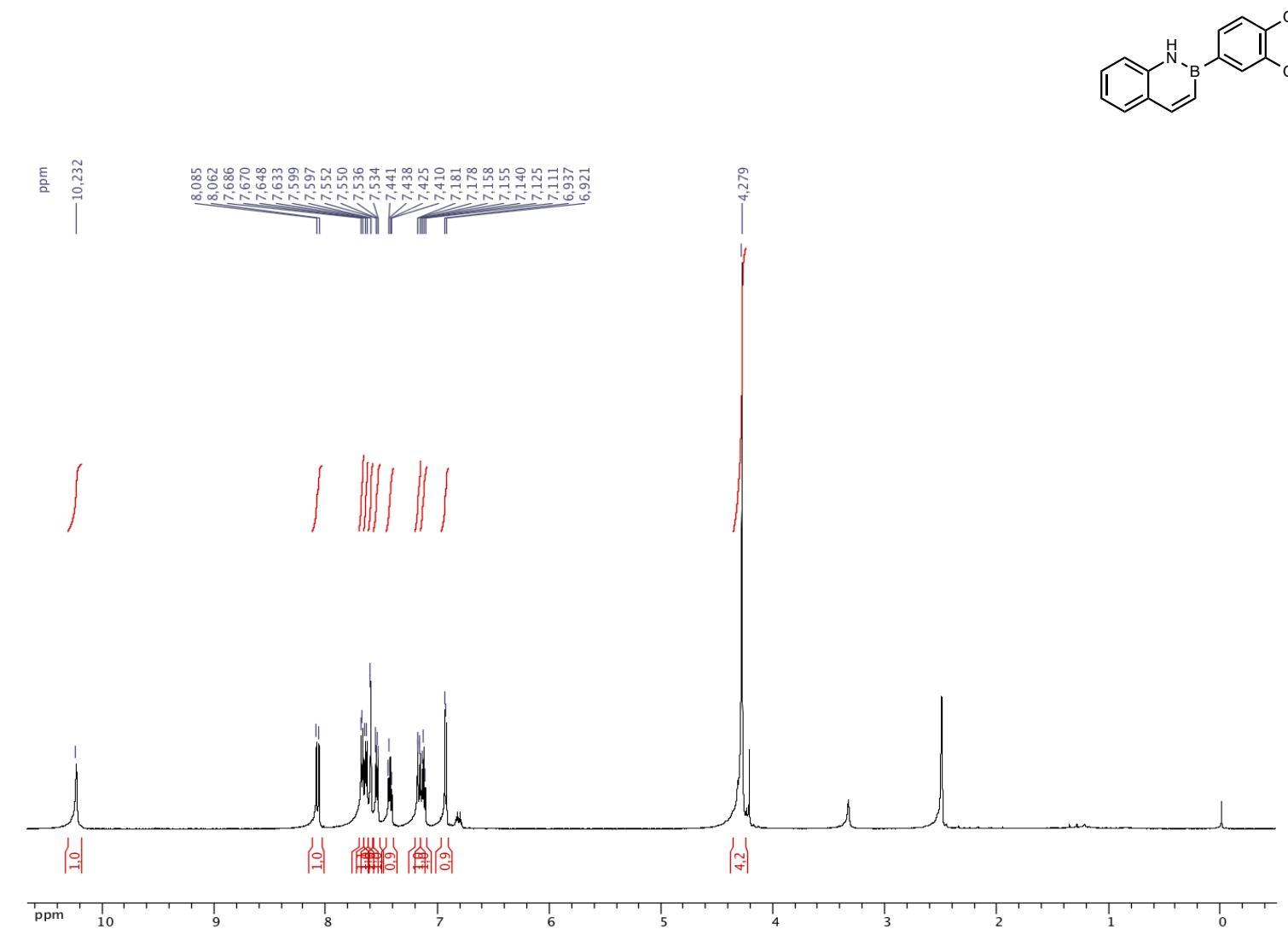

${ }^{1} \mathrm{H}$ NMR (DMSO- $d_{6}, 500.4 \mathrm{MHz}$ ) of 2-(2,3-dihydro-1,4-benzodioxin-6-yl)-2,1-borazanaphthalene (10b)
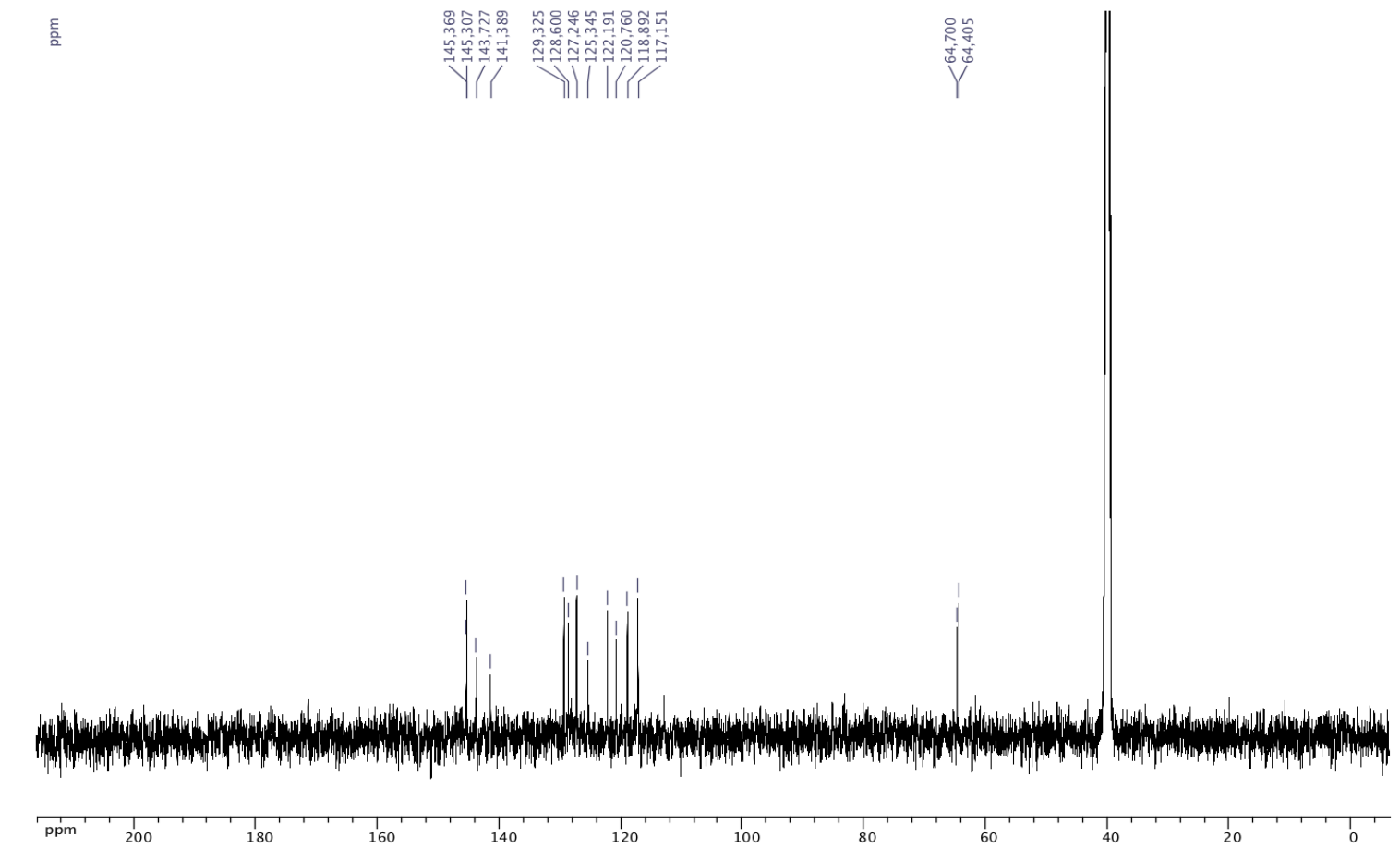

${ }^{13} \mathrm{C}\left\{{ }^{1} \mathrm{H}\right\}$ NMR (DMSO- $d_{6}, 125.8 \mathrm{MHz}$ ) of 2-(2,3-dihydro-1,4-benzodioxin-6-yl)-2,1-borazanaphthalene (10b) 


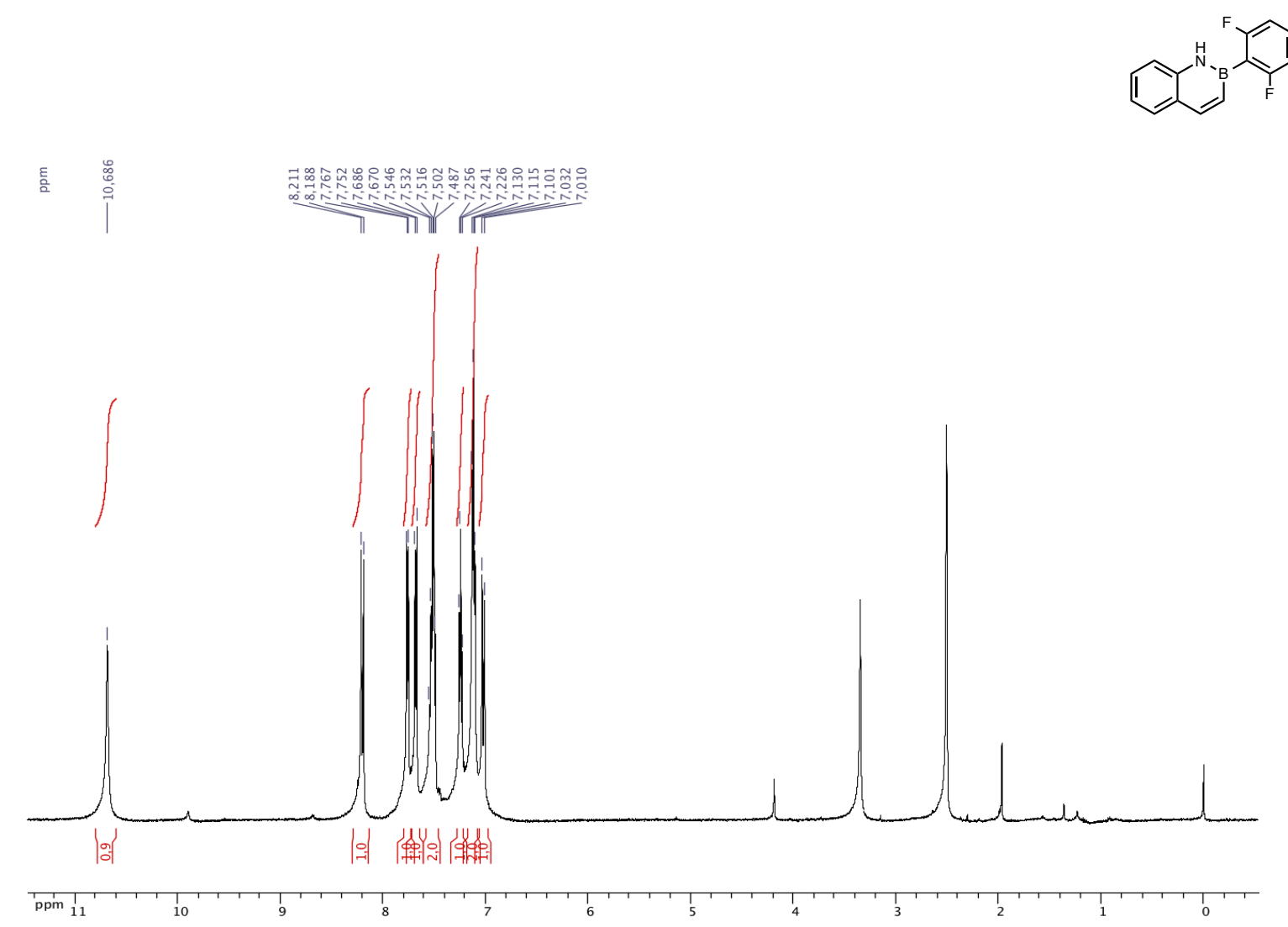

${ }^{1} \mathrm{H}$ NMR (DMSO- $d_{6}, 500.4 \mathrm{MHz}$ ) of 2-(2,6-difluorophenyl)-2,1-borazanaphthalene (10c)
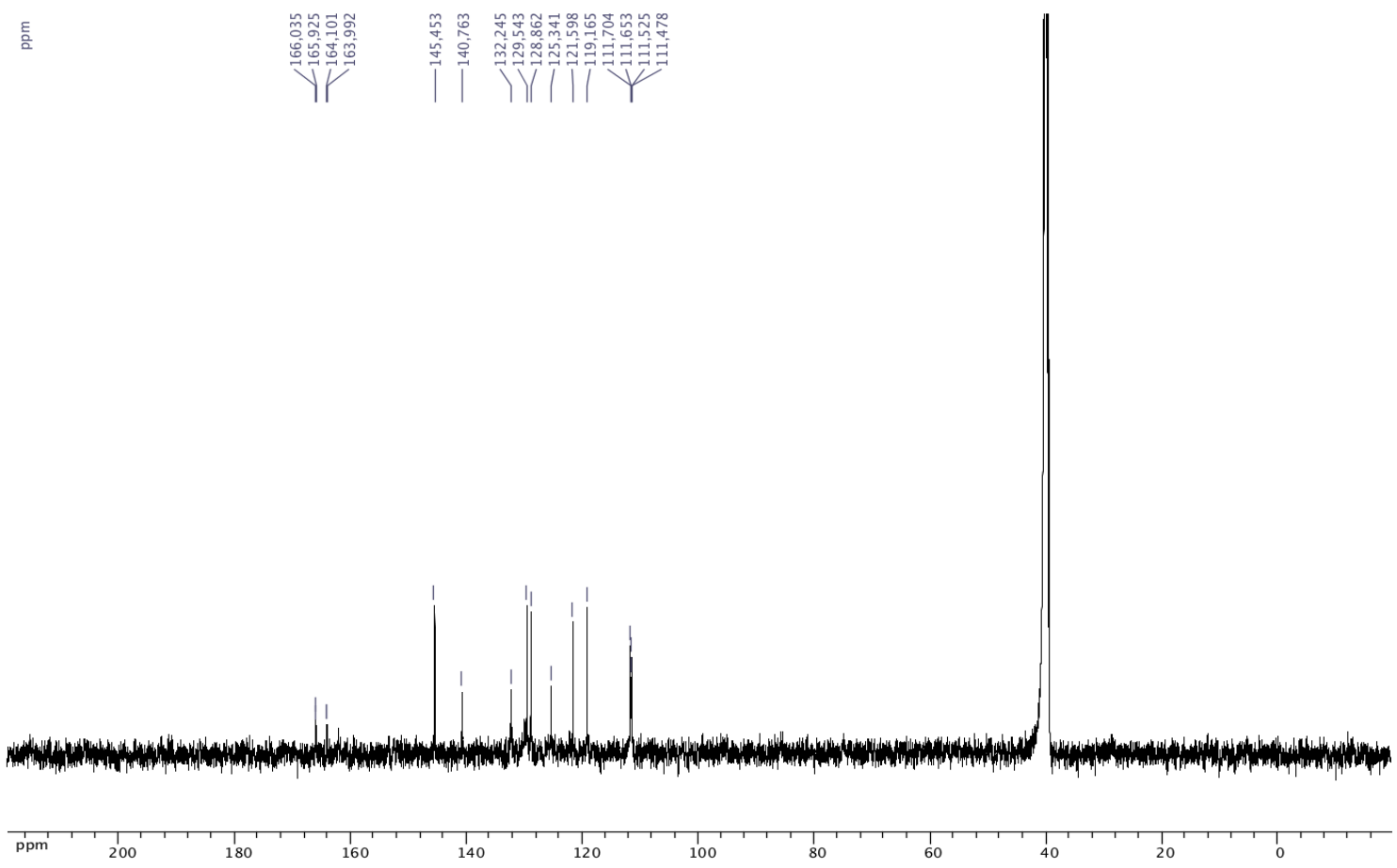

${ }^{13} \mathrm{C}\left\{{ }^{1} \mathrm{H}\right\}$ NMR (DMSO- $d_{6}, 125.8 \mathrm{MHz}$ ) of 2-(2,6-difluorophenyl)-2,1-borazanaphthalene (10c) 


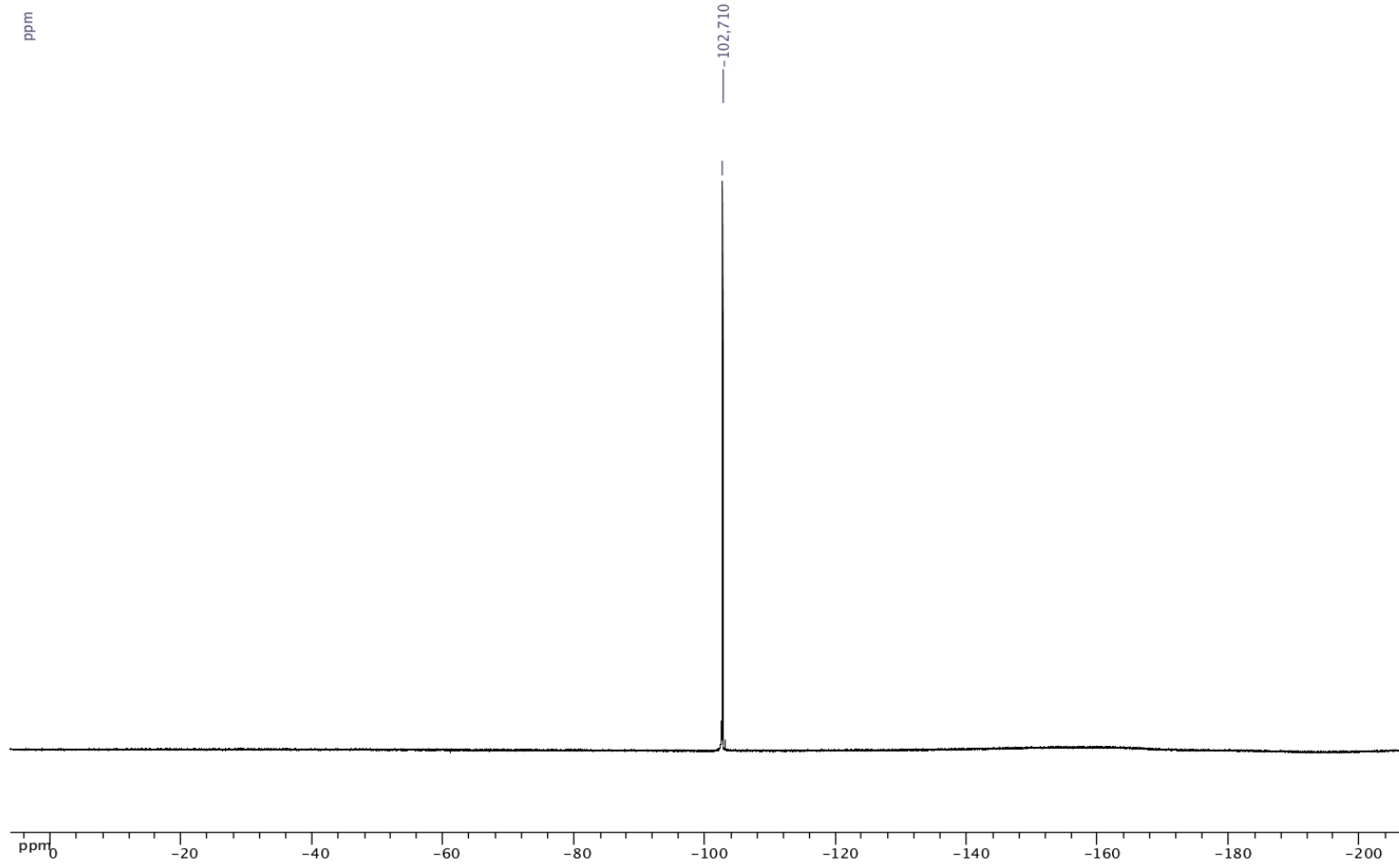

${ }^{19} \mathrm{~F}\left\{{ }^{1} \mathrm{H}\right\}$ NMR (DMSO- $d_{6}, 470.8 \mathrm{MHz}$ ) of 2-(2,6-difluorophenyl)-2,1-borazanaphthalene (10c) 


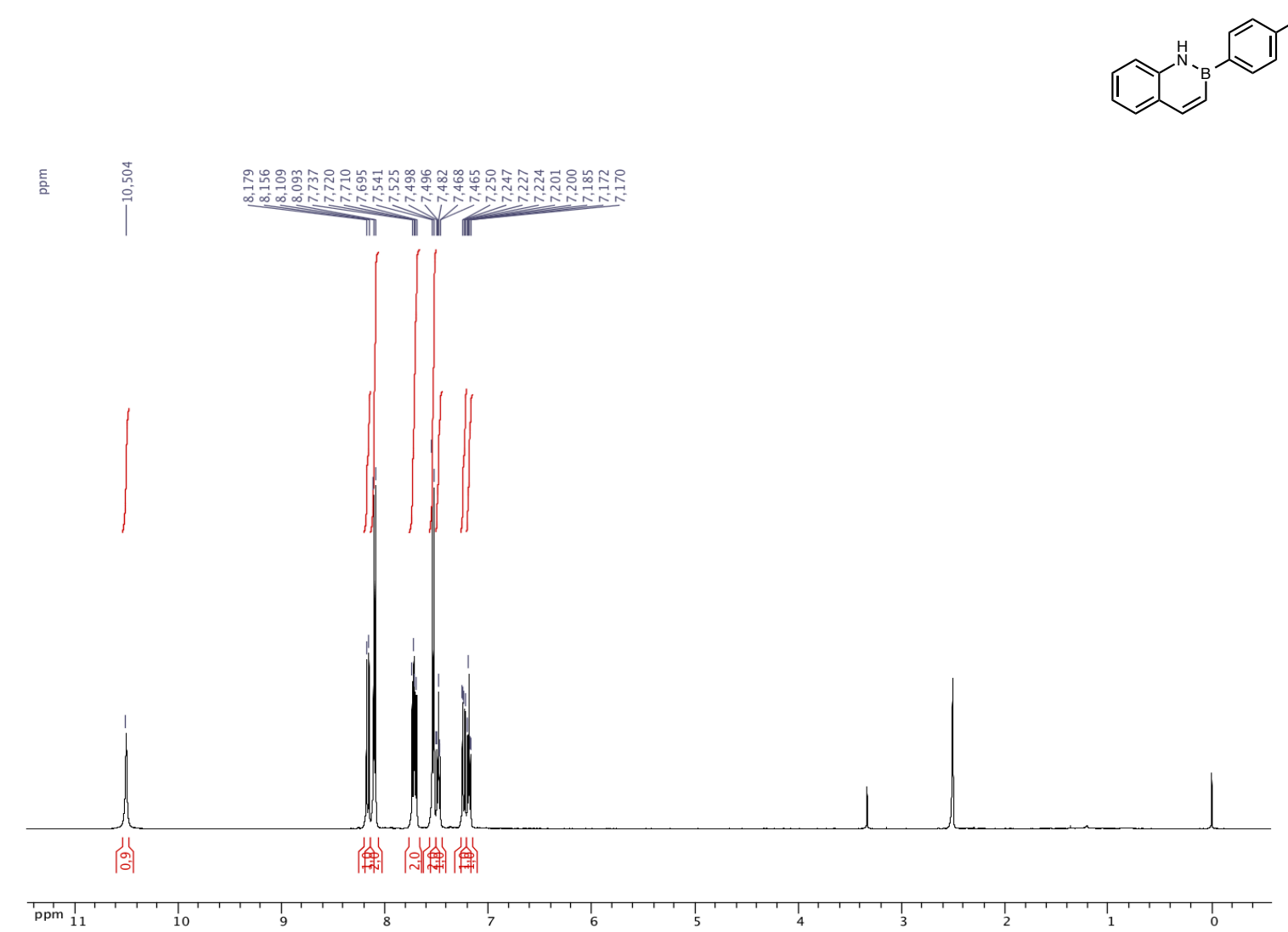

${ }^{1} \mathrm{H}$ NMR (DMSO- $d_{6}, 500.4 \mathrm{MHz}$ ) of 2-(4-chlorophenyl)-2,1-borazanaphthalene (10d)

言
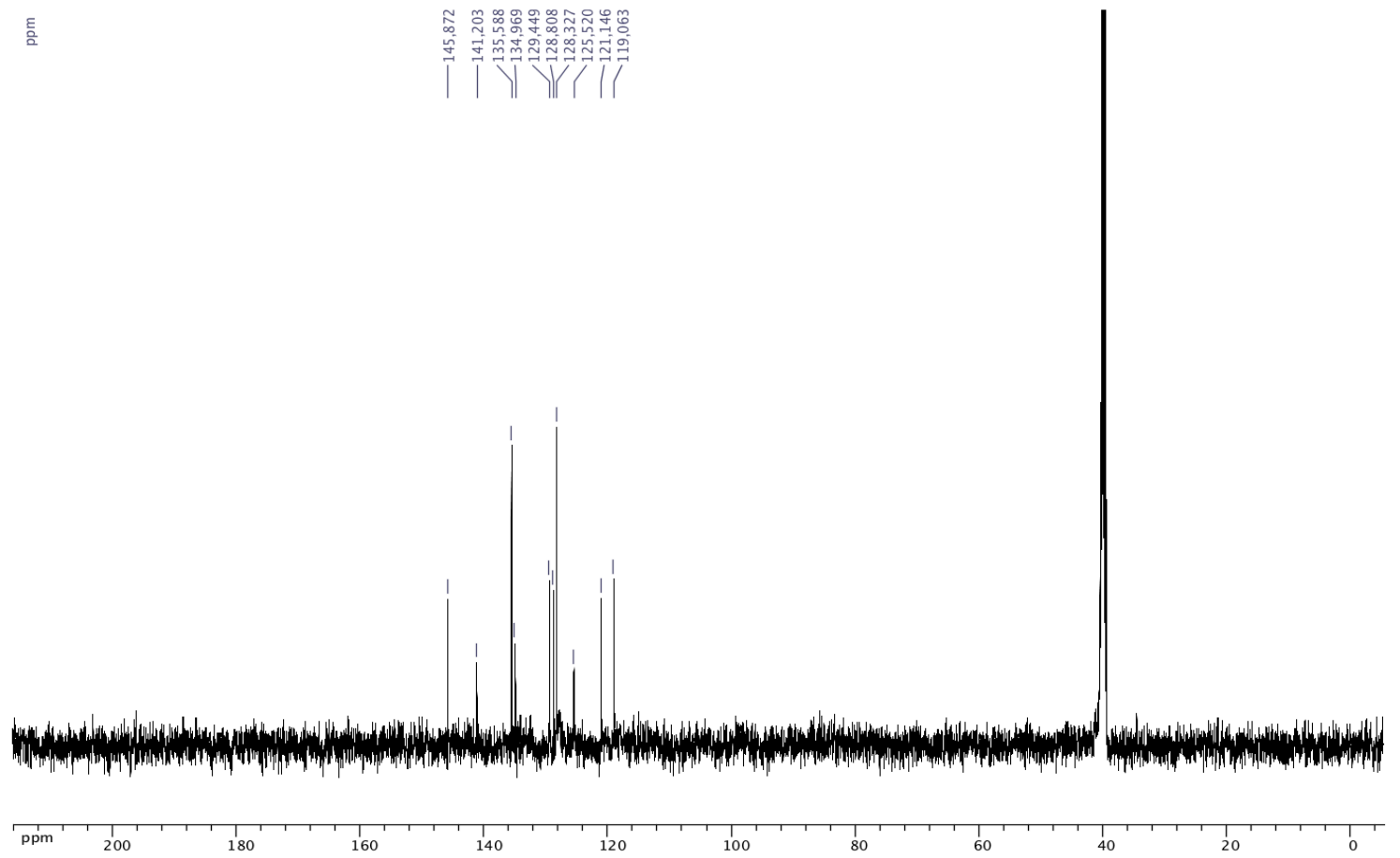

${ }^{13} \mathrm{C}\left\{{ }^{1} \mathrm{H}\right\}$ NMR (DMSO- $\left.d_{6}, 125.8 \mathrm{MHz}\right)$ of 2-(4-chlorophenyl)-2,1-borazanaphthalene (10d) 


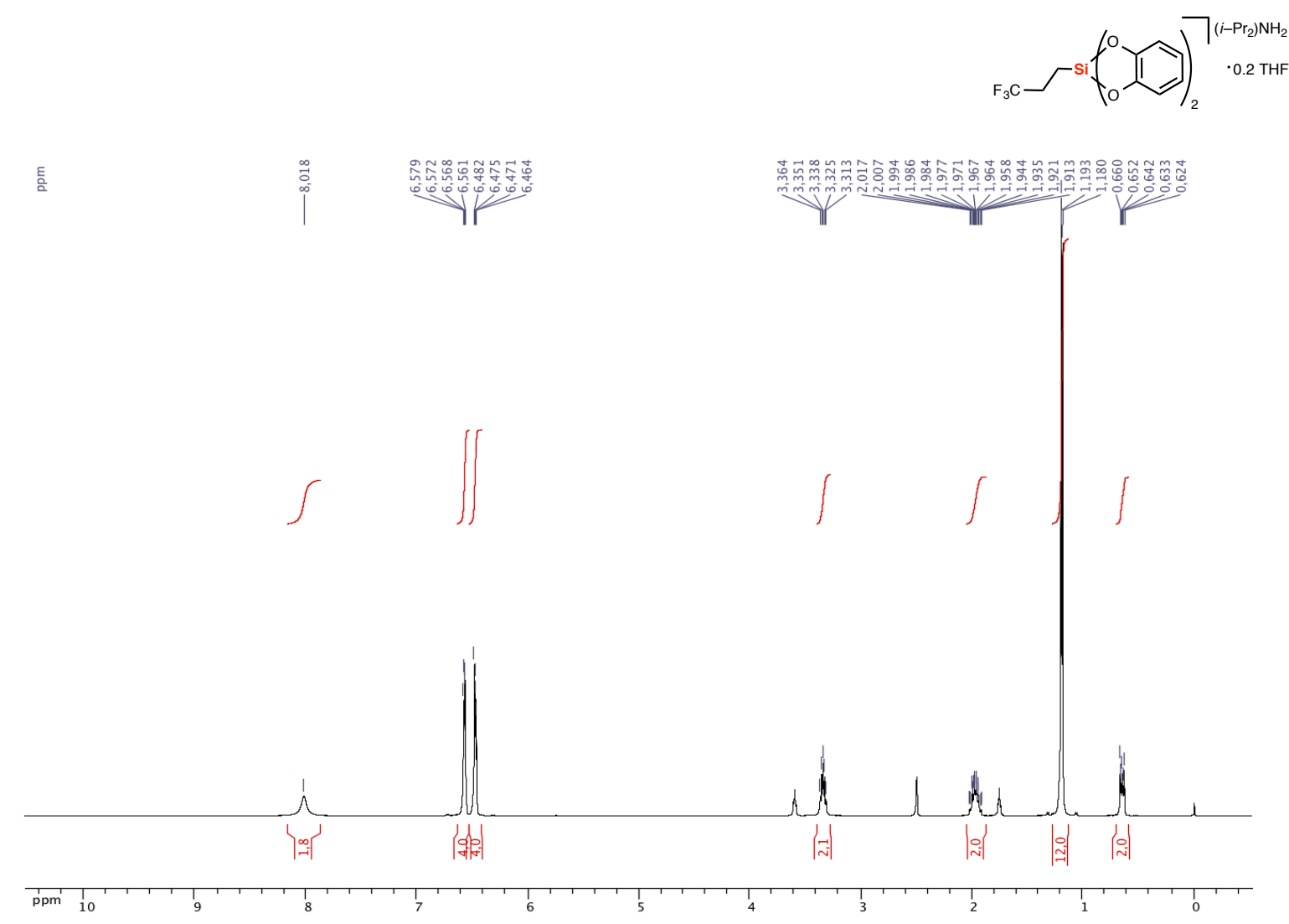

${ }^{1} \mathrm{H}$ NMR (DMSO- $d_{6}, 500.4 \mathrm{MHz}$ ) of diisopropylammonium 3,3,3-trifluoropropylbis(catecholato)silicate (11a)
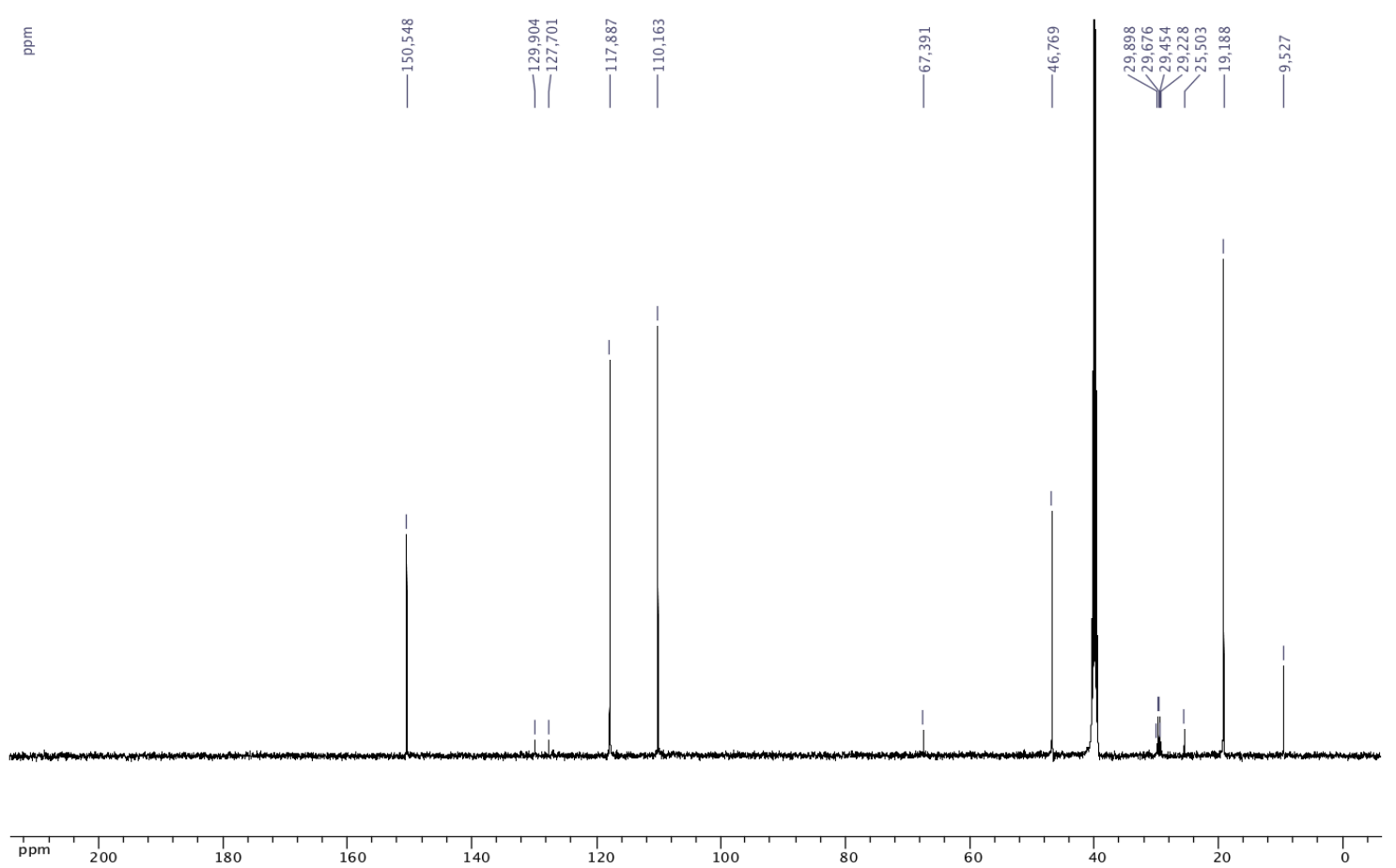

${ }^{13} \mathrm{C}\left\{{ }^{1} \mathrm{H}\right\}$ NMR (DMSO- $d_{6}, 125.8 \mathrm{MHz}$ ) of diisopropylammonium 3,3,3-trifluoropropylbis(catecholato)silicate (11a) 


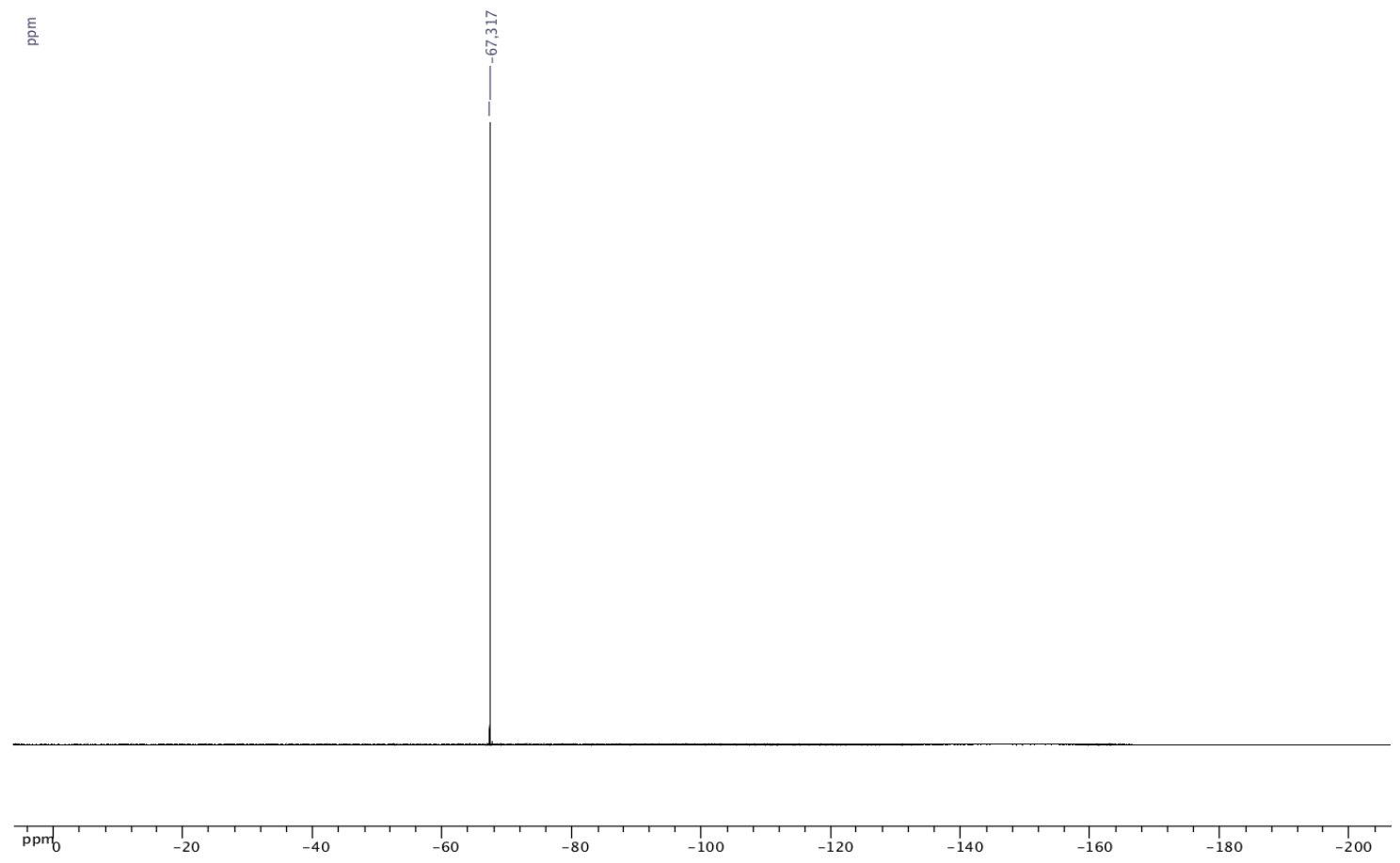

${ }^{19} \mathrm{~F}\left\{{ }^{1} \mathrm{H}\right\}$ NMR (DMSO- $d_{6}, 470.8 \mathrm{MHz}$ ) of diisopropylammonium 3,3,3-trifluoropropylbis(catecholato)silicate (11a) 


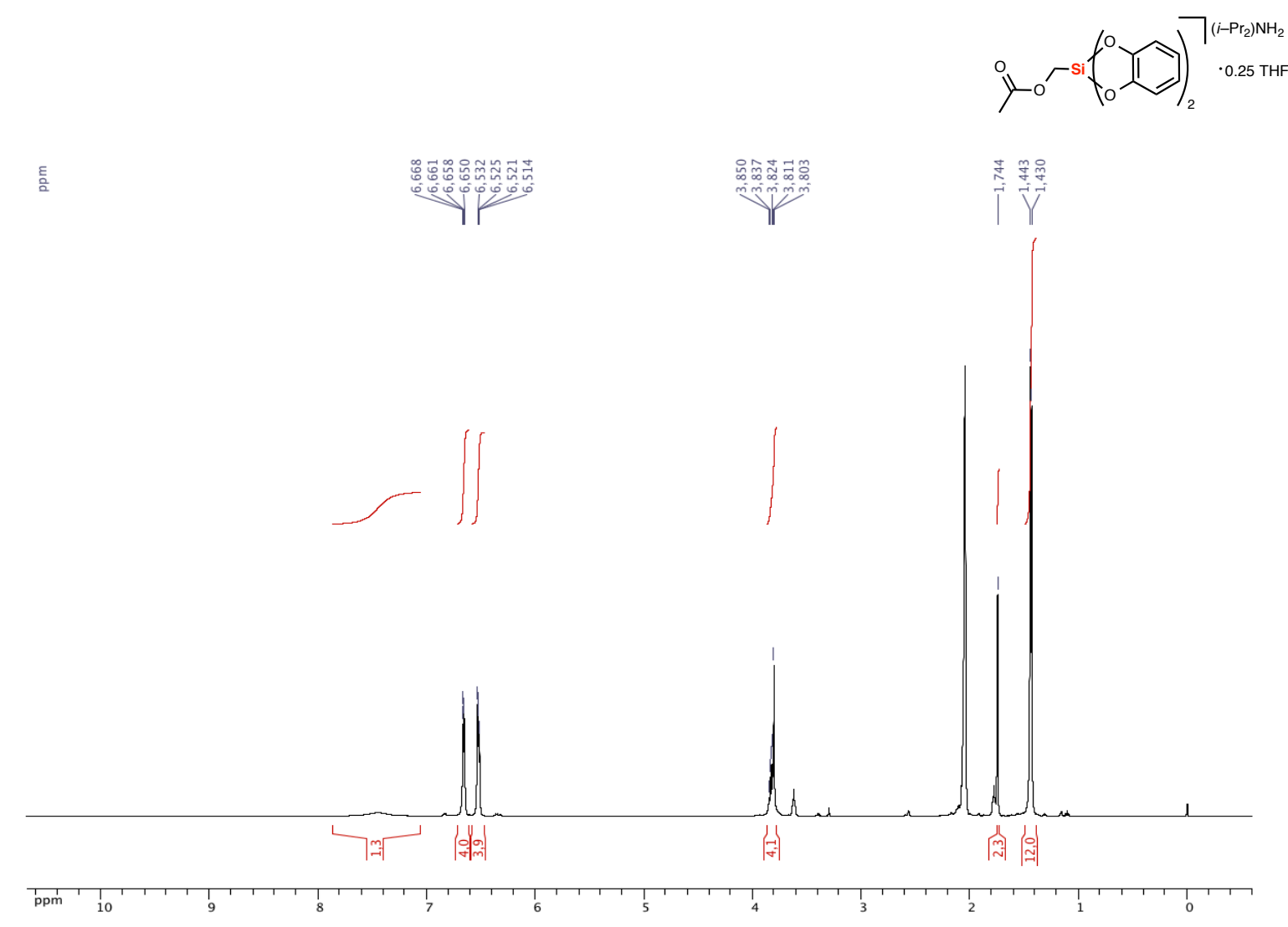

${ }^{1} \mathrm{H}$ NMR (acetone- $d_{6}, 500.4 \mathrm{MHz}$ ) of diisopropylammonium acetoxymethylbis(catecholato)silicate (11b)
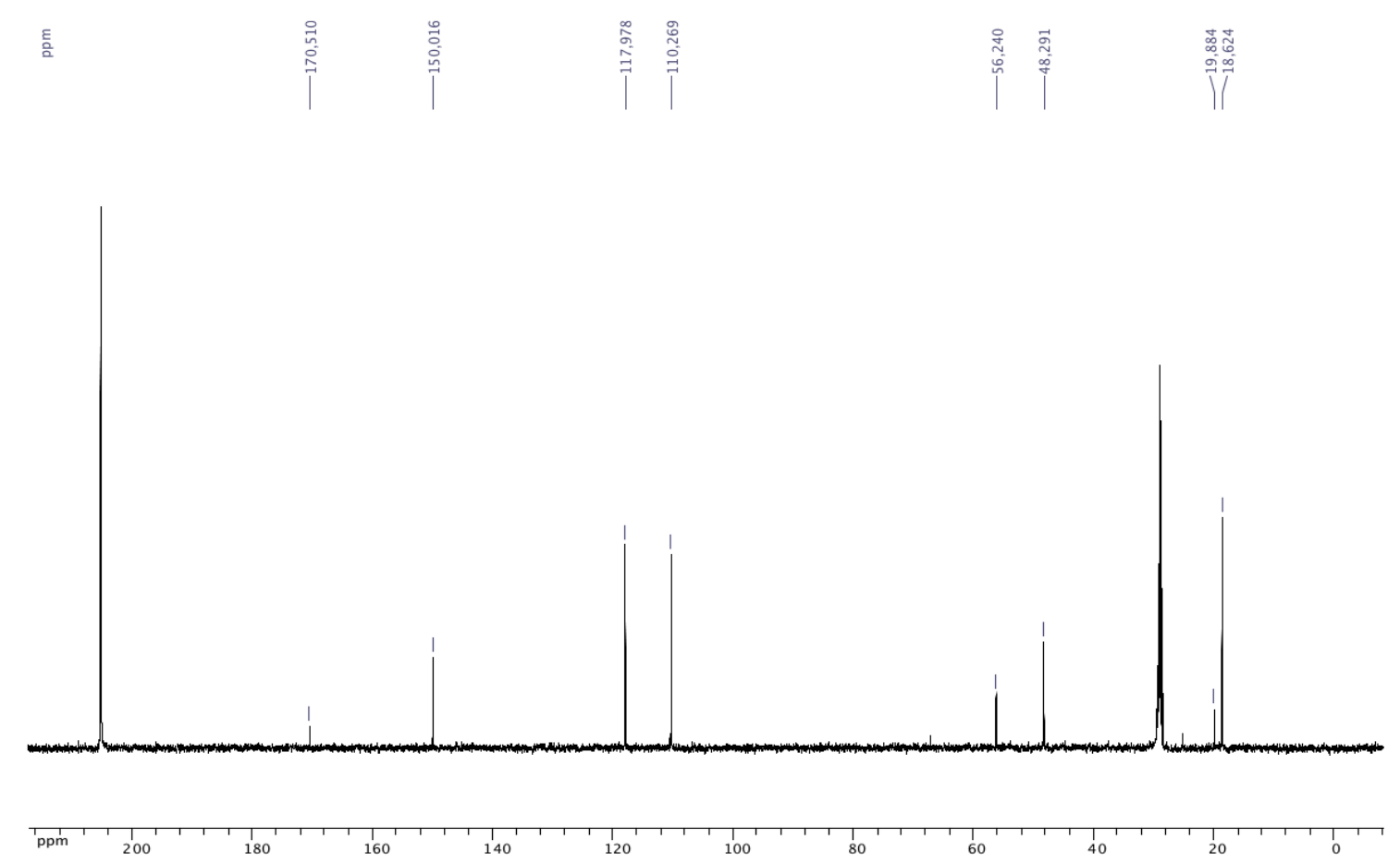

${ }^{13} \mathrm{C}\left\{{ }^{1} \mathrm{H}\right\}$ NMR (acetone- $d_{6}, 125.8 \mathrm{MHz}$ ) of diisopropylammonium acetoxymethylbis(catecholato)silicate (11b) 


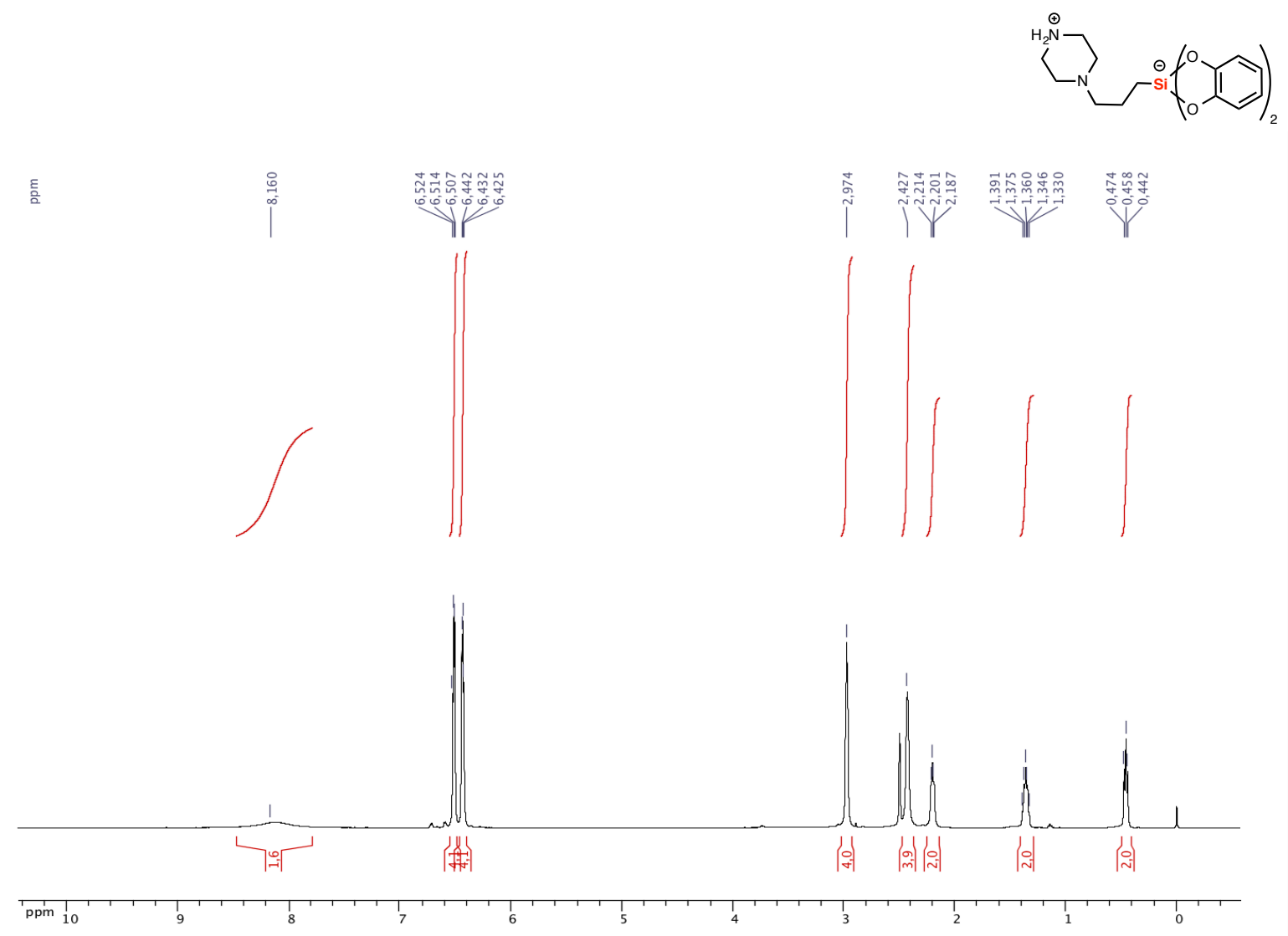

${ }^{1} \mathrm{H}$ NMR (DMSO- $d_{6}, 500.4 \mathrm{MHz}$ ) of 3-(piperazin-1-ium-1-yl)propylbis(catecholato)silicate (11c)

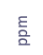

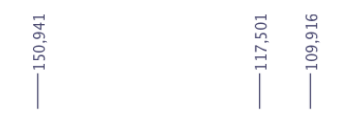

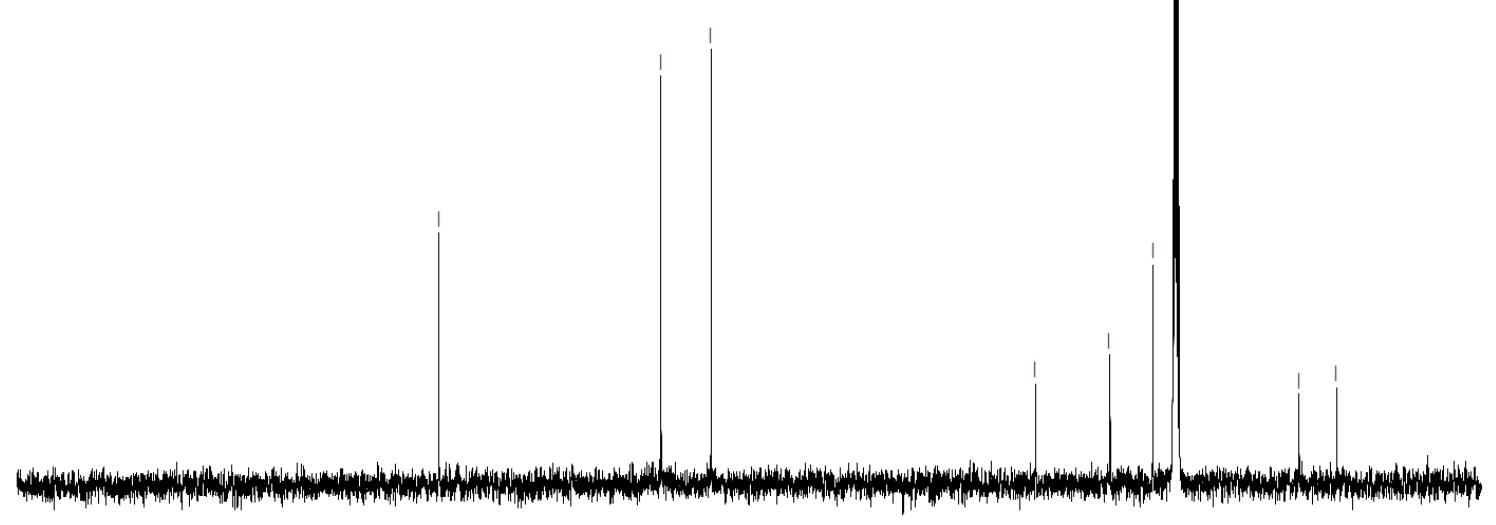

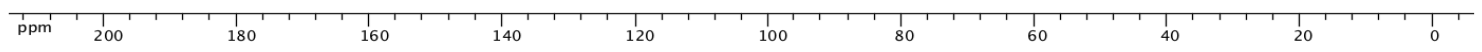

${ }^{13} \mathrm{C}\left\{{ }^{1} \mathrm{H}\right\}$ NMR (DMSO-d, $125.8 \mathrm{MHz}$ ) of 3-(piperazin-1-ium-1-yl)propylbis(catecholato)silicate (11c) 


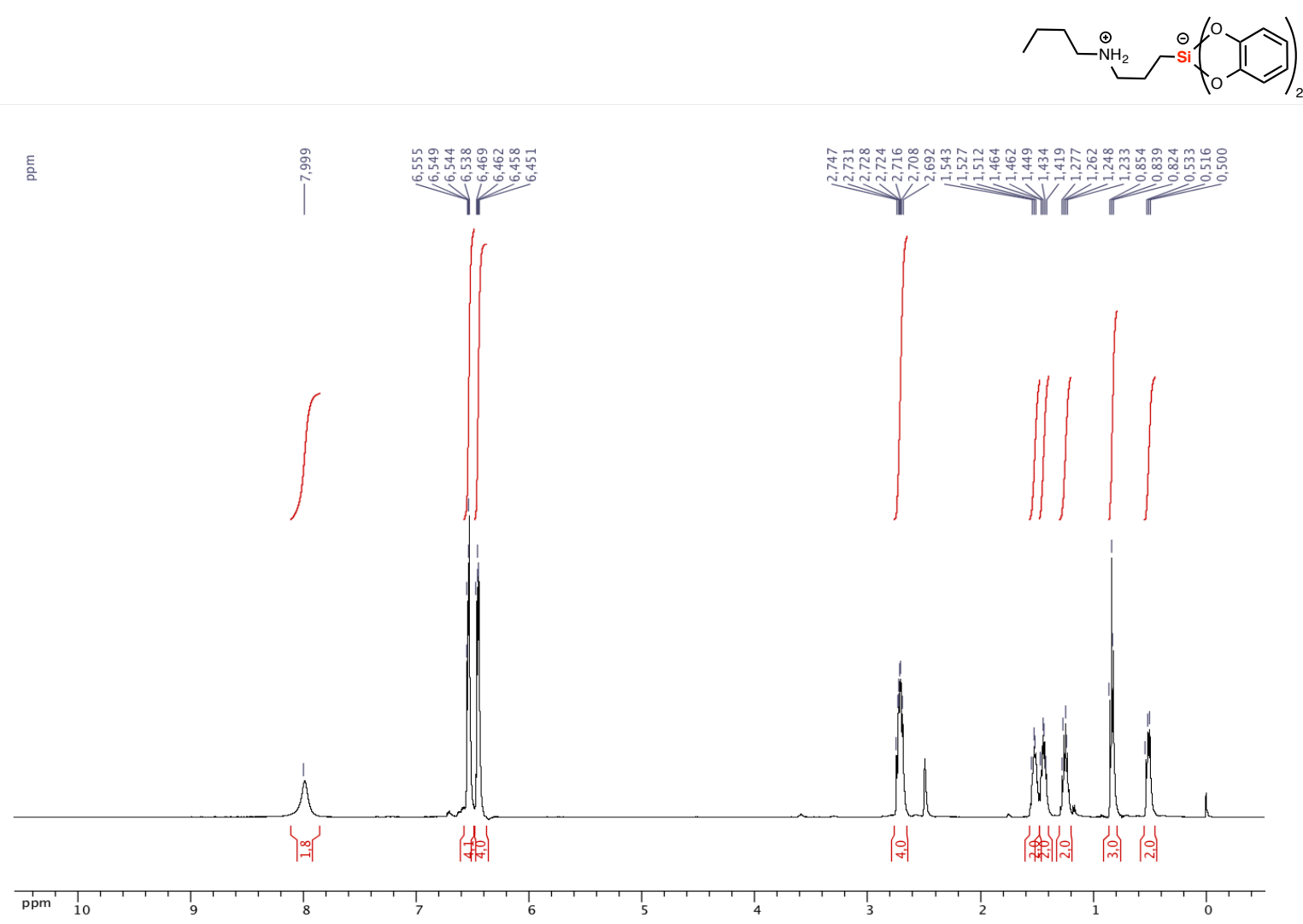

${ }^{1} \mathrm{H}$ NMR (DMSO- $d_{6}, 500.4 \mathrm{MHz}$ ) of 3-(butylammonio)propylbis(catecholato)silicate (11d)
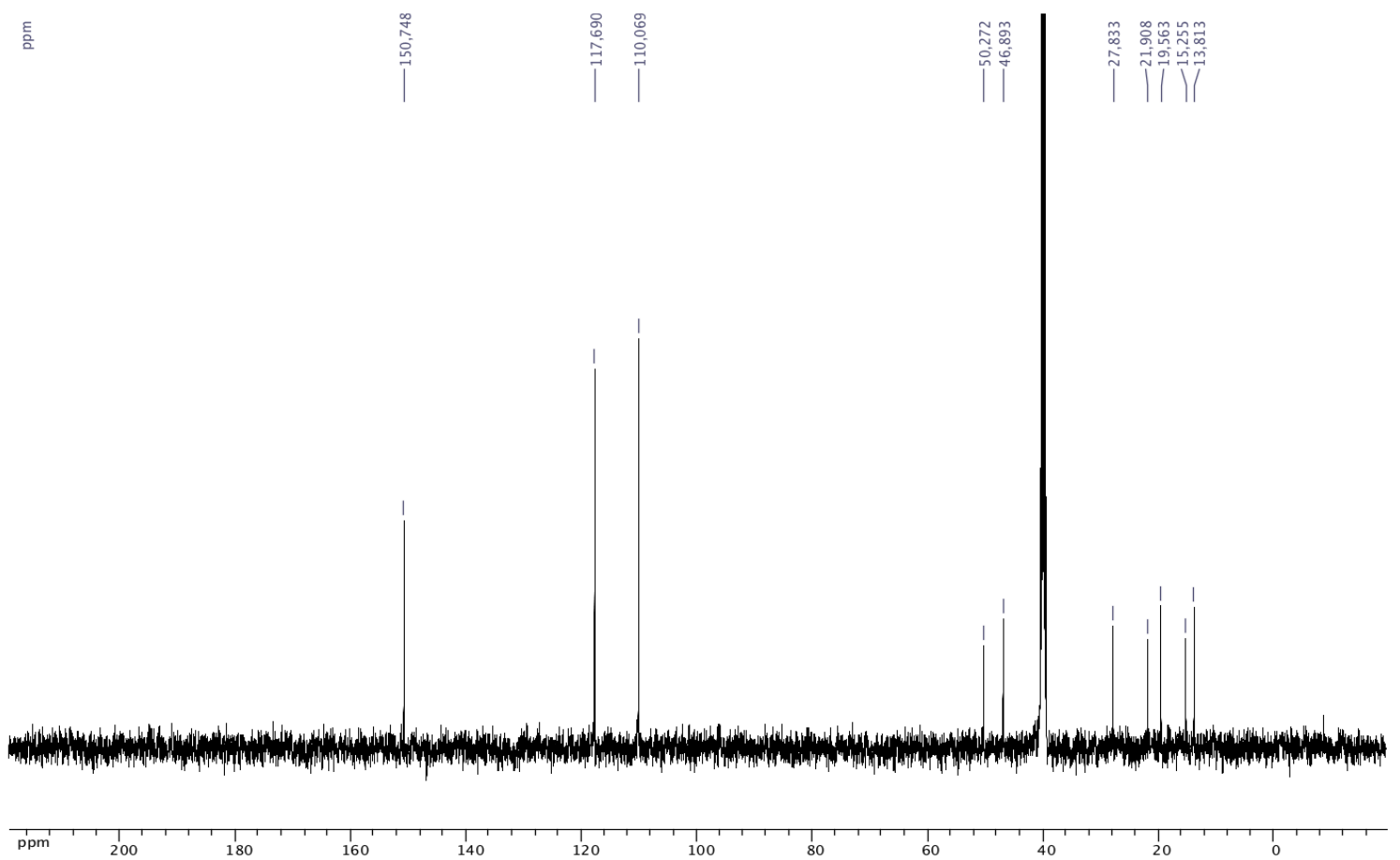

${ }^{13} \mathrm{C}\left\{{ }^{1} \mathrm{H}\right\}$ NMR (DMSO- $d_{6}, 125.8 \mathrm{MHz}$ ) of 3-(butylammonio)propylbis(catecholato)silicate (11d) 